Supporting Information: Hydrogen-Bonding Interaction Regulates Photoisomerization of a Single-Bond-Rotation Locked Photoactive Yellow Protein Chromophore in Protein

Teng-Shuo Zhang, Ye-Guang Fang, Xiu-Fang Song, Wei-Hai Fang, and Ganglong Cui*

Key Laboratory of Theoretical and Computational Photochemistry, Ministry of Education, Chemistry College, Beijing Normal University, Beijing 100875, P. R. China

\title{
Email: ganglong.cui@bnu.edu.cn
}

Table of Contents

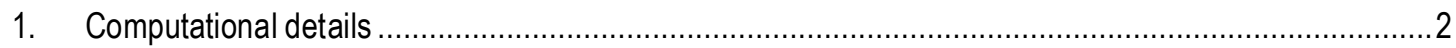

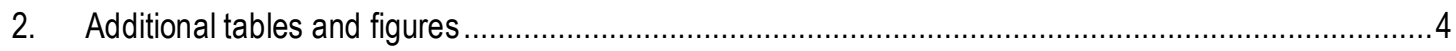

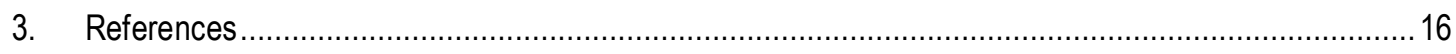

4. Cartesian coordinates of all optimized structures (QM region) ............................................... 19 


\section{Computational details}

The crystal structure of the wild-type PYP protein (code: 5HD3) is initially obtained from the RCSB protein data bank (PDB). ${ }^{1}$ In this wild type PYP protein, the natural PYP chromophore is manually modified into the single-bond-rotation locked chromophore according to recent experiments. This modified protein structure is solvated in an $80 \times 80 \times 80 \AA^{3}$ cubic water box. 6 sodium ions are added to neutralize the system. Subsequently, the added ions and water molecules are first minimized; then, the entire protein system is minimized (1000 steps), heated (10 ps), and equilibrated (1 ns, NPT: $300 \mathrm{~K}, 1 \mathrm{~atm})$. In the MD simulations, the force-field parameters of the single-bond-rotation locked PYP chromophore are generated based on the generalized Amber force field (GAFF), ${ }^{2}$ those of residues are described with the Amber ff 99 force field, and water molecules are described using the TIP3P model ${ }^{3}$ in the AMBER package. ${ }^{4} \mathrm{~A}$ cutoff of $10 \AA$ is employed to truncate the nonbonding interactions. The final MD snapshot is truncated into a spherical ball with a radius of $35 \AA$ and is chosen as the starting structure for the following QM/MM electronic structure calculations.

All electronic structure calculations for the single-bond-rotation locked PYP chromophore in the protein surroundings are carried out at the QM/MM level.5,6 The QM subsystem contains the PYP chromophore and two amino acid residues (Tyr42 and Glu46) that are hydrogen bonded to the chromophore; whereas, the MM subsystem consists of the remaining protein structure and all ions and water molecules. Hydrogen link atoms are used in combination with the charge-shift scheme at the QM/MM boundary.7-9 The electrostatic embedding scheme is adopted in our QM/MM calculations. In geometry optimizations, water molecules that are 30 angstroms away from the chromophore are fixed and the remaining water molecules and protein residues are set 
to free. The MM subsystem is treated with the same force fields (see above). The state-averaged complete active space self-consistent field (CASSCF) ${ }^{10}$ method is chosen as the QM electronic structure method to optimize minima and conical intersections. Since the CASSCF method does not adequately capture electronic correlation energy, the complete active space second-order perturbation approach (CASPT2) ${ }^{11,12}$ is utilized to re-evaluate single-point energies of all optimized structures and paths.

In CASSCF and CASPT2 calculations, a large active space of 14 electrons in 12 orbitals is used. The ANO-RCC-VDZP basis set is used for all atoms in the QM region throughout all QM/MM calculations. ${ }^{13,14}$ The Cholesky decomposition technique with unbiased auxiliary basis sets is used for accurate two-electron integral approximations. ${ }^{15,16}$ The imaginary shift technique $(0.2 \mathrm{au})$ is used to avoid the intruder-state issue. ${ }^{17}$ The ionization potential electron affinity shift is set to zero, which has been demonstrated to work well for organic chromophores. ${ }^{18,19}$ All QM(CASPT2//CASSCF)/MM calculations are carried out using the OpenMolcas package ${ }^{20}$ that interfaces with the TINKER 6.3.3 package. ${ }^{21}$ 


\section{Additional tables and figures}

Table S1. QM(CASPT2//CASSCF)/MM calculated energies and related electronic configurations for the PYP chromophore in the wild-type protein.

\begin{tabular}{|c|c|c|c|c|}
\hline \multirow[t]{2}{*}{ State } & \multirow[t]{2}{*}{ Root } & \multicolumn{2}{|c|}{ Energy(kcal/mol) } & \multirow[t]{2}{*}{ configurations (weight) } \\
\hline & & CAS & CASPT2 & \\
\hline \multirow[t]{2}{*}{ SO-MIN } & Root1 & 0.0 & 0.0 & $222222200000(0.85)$ \\
\hline & Root2 & 85.0 & 62.6 & 222222ud0000(0.71) \\
\hline \multirow[t]{2}{*}{ SO-cisT-MIN } & Root1 & 23.8 & 24.5 & $222222200000(0.85)$ \\
\hline & Root2 & 98.3 & 80.7 & 222222ud0000(0.72) \\
\hline \multirow[t]{2}{*}{ S0-cisP-MIN } & Root1 & 23.7 & 25.2 & $222222200000(0.85)$ \\
\hline & Root2 & 106.8 & 89.0 & 222222ud0000(0.71) \\
\hline \multirow[t]{2}{*}{ S1-MIN } & Root1 & 5.1 & 3.7 & $222222200000(0.85)$ \\
\hline & Root2 & 77.3 & 60.3 & $222222 \mathrm{ud} 0000(0.71)$ \\
\hline \multirow[t]{2}{*}{ S1-CT1-MIN } & Root1 & 53.1 & 44.2 & $222222200000(0.79)$ \\
\hline & Root2 & 65.5 & 56.1 & $222222 \mathrm{ud} 0000(0.80)$ \\
\hline \multirow[t]{2}{*}{ S1-CT2-MIN } & Root1 & 63 & 54.9 & $222222200000(0.77)$ \\
\hline & Root2 & 73.6 & 64.5 & 222222ud0000(0.80) \\
\hline \multirow[t]{2}{*}{ S1S0-1 } & Root1 & 67.6 & 55.0 & $222222200000(0.80)$ \\
\hline & Root2 & 68.0 & 56.5 & 222222ud0000(0.81) \\
\hline \multirow[t]{4}{*}{ S1S0-2 } & Root1 & 74.4 & 60.8 & $222222200000(0.52)$ \\
\hline & & & & 222222ud0000(0.24) \\
\hline & Root2 & 74.4 & 62.0 & $222222200000(0.25)$ \\
\hline & & & & 222222ud0000(0.56) \\
\hline
\end{tabular}

Table S2. QM(CASSCF)/MM calculated Mulliken charges of selected atoms for the PYP chromophore in the wild-type protein.

\begin{tabular}{lllllll}
\hline State & 01 & C2 & 013 & C12 & C11 & C10 \\
\hline S0-MIN & -0.83 & 0.39 & -0.47 & 0.34 & -0.10 & 0.16 \\
\hline S0-cisT-MIN & -0.83 & 0.39 & -0.44 & 0.34 & -0.13 & 0.19 \\
\hline S0-cisP-MIN & -0.81 & 0.40 & -0.40 & 0.34 & -0.15 & 0.18 \\
\hline S1-FC & -0.72 & 0.40 & -0.54 & 0.24 & -0.12 & 0.04 \\
\hline S1-MIN & -0.71 & 0.37 & -0.56 & 0.27 & -0.06 & 0.04 \\
\hline S1-CT1-MIN & -0.90 & 0.33 & -0.42 & 0.36 & 0.17 & -0.07 \\
\hline S1-CT2-MIN & -0.88 & 0.34 & -0.41 & 0.34 & 0.21 & -0.11 \\
\hline
\end{tabular}


Table S3. QM(CASPT2//CASSCF)/MM calculated energies and related electronic configurations for the PYP chromophore in the R52A mutant protein.

\begin{tabular}{|c|c|c|c|c|}
\hline \multirow[t]{2}{*}{ States } & \multirow[t]{2}{*}{ Root } & \multicolumn{2}{|c|}{ Energy(kcal/mol) } & \multirow[t]{2}{*}{ configurations (weight) } \\
\hline & & CAS & CASPT2 & \\
\hline \multirow[t]{2}{*}{ SO-MIN } & Root1 & 0.0 & 0.0 & $222222200000(0.85)$ \\
\hline & Root2 & 86.1 & 62.8 & 222222ud0000(0.72) \\
\hline \multirow[t]{2}{*}{ S0-cisT-MIN } & Root1 & 22.0 & 21.5 & $222222200000(0.85)$ \\
\hline & Root2 & 93.4 & 76.0 & 222222ud0000(0.71) \\
\hline \multirow[t]{2}{*}{ S0-cisP-MIN } & Root1 & 22.8 & 24.1 & $222222200000(0.85)$ \\
\hline & Root2 & 104.8 & 87.7 & 222222ud0000(0.72) \\
\hline \multirow[t]{2}{*}{ S1-MIN } & Root1 & 6.5 & 4.8 & $222222200000(0.85)$ \\
\hline & Root2 & 78.3 & 61.3 & 222222ud0000(0.71) \\
\hline \multirow[t]{2}{*}{ S1-CT1-MIN } & Root1 & 54.7 & 44.2 & $222222200000(0.81)$ \\
\hline & Root2 & 66.5 & 57.6 & 222222ud0000(0.80) \\
\hline \multirow[t]{2}{*}{ S1-CT2-MIN } & Root1 & 63.5 & 53.4 & $222222200000(0.79)$ \\
\hline & Root2 & 74.3 & 66.0 & 222222ud0000(0.80) \\
\hline \multirow[t]{2}{*}{ S1S0-1 } & Root1 & 67.0 & 56.3 & $222222200000(0.80)$ \\
\hline & Root2 & 69.8 & 59.0 & 222222ud0000(0.80) \\
\hline \multirow[t]{2}{*}{ S1S0-2 } & Root1 & 73.9 & 63.2 & $222222200000(0.76)$ \\
\hline & Root2 & 77.1 & 66.7 & 222222ud0000(0.80) \\
\hline
\end{tabular}

Table S4. QM(CASSCF)/MM calculated Mulliken charges of selected atoms for the PYP chromophore in R52A mutated protein.

\begin{tabular}{lllllll}
\hline State & 01 & C2 & 013 & C12 & C11 & C10 \\
\hline S0-MIN & -0.86 & 0.39 & -0.47 & 0.34 & -0.10 & 0.16 \\
\hline S0-CisT-MIN & -0.84 & 0.40 & -0.47 & 0.33 & -0.14 & 0.19 \\
\hline S0-cisP-MIN & -0.83 & 0.40 & -0.41 & 0.34 & -0.15 & 0.18 \\
\hline S1-FC & -0.74 & 0.40 & -0.53 & 0.23 & -0.12 & 0.03 \\
\hline S1-MIN & -0.73 & 0.38 & -0.55 & 0.26 & -0.06 & 0.03 \\
\hline S1-CT1-MIN & -0.92 & 0.33 & -0.41 & 0.36 & 0.17 & -0.07 \\
\hline S1-CT2-MIN & -0.91 & 0.34 & -0.40 & 0.34 & 0.21 & -0.11
\end{tabular}




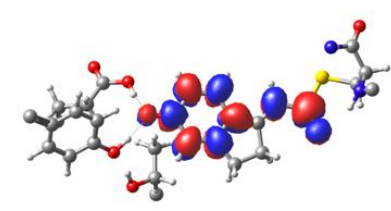

123

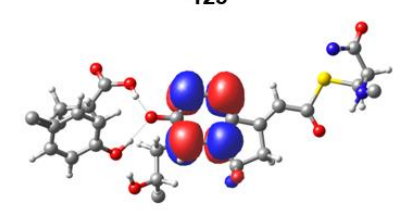

120

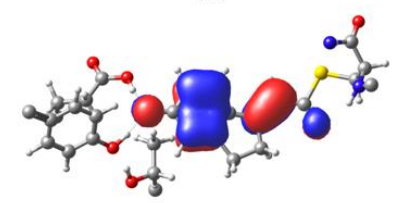

117

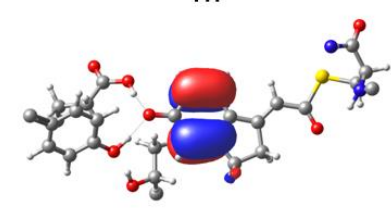

114

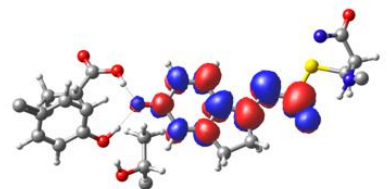

122

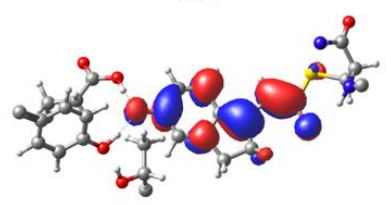

LUMO 119

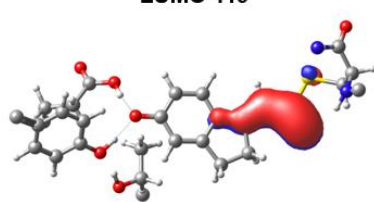

116

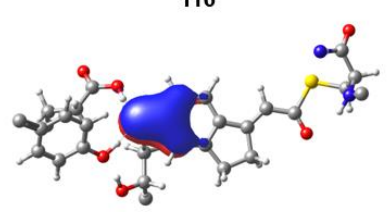

113

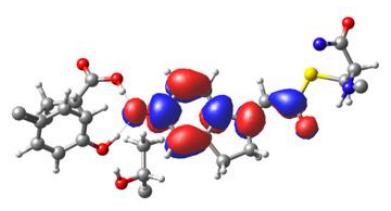

121

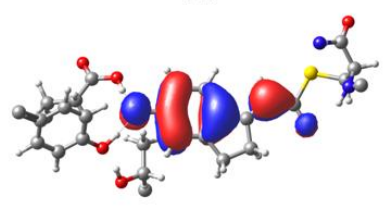

HОMO 118

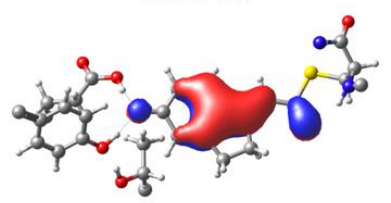

115

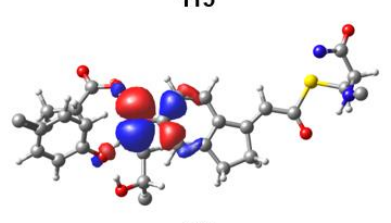

112

Figure S1. Molecular orbitals used as the active space of 14 electrons in 12 orbitals in the CASSCF and CASPT2 calculations.

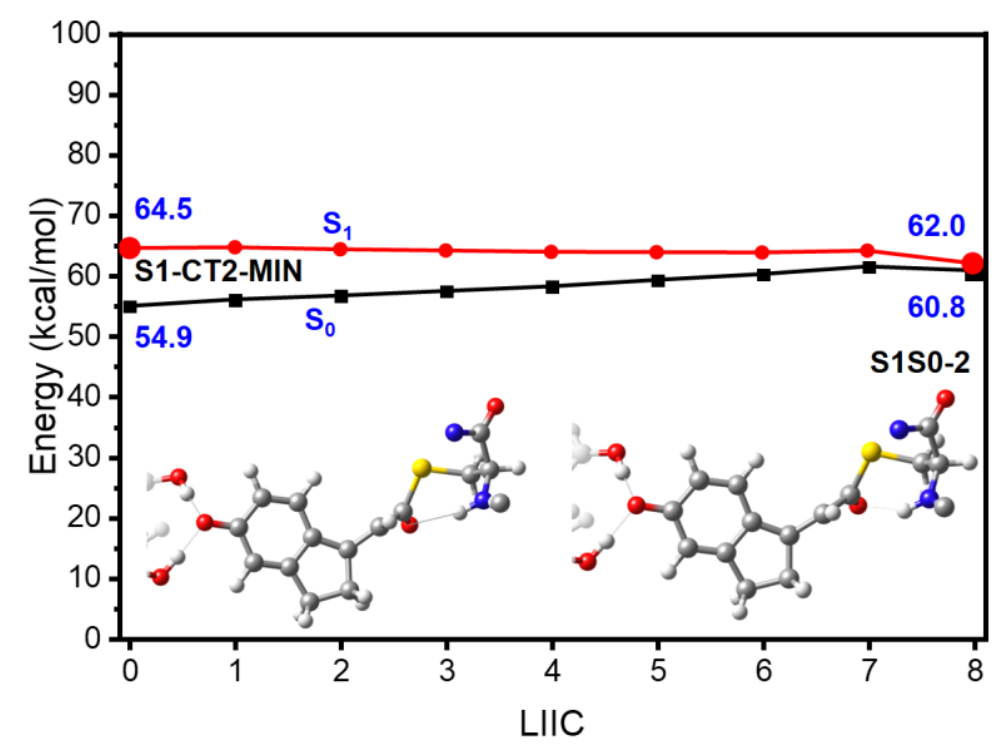

Figure S2. QM(CASPT2//CASSCF)/MM calculated linearly interpolated internal coordinate (LIIC) paths connecting S1-CT2-MIN and S1S0-2 of the single-bond-rotation locked PYP chromophore in the wild-type protein. 


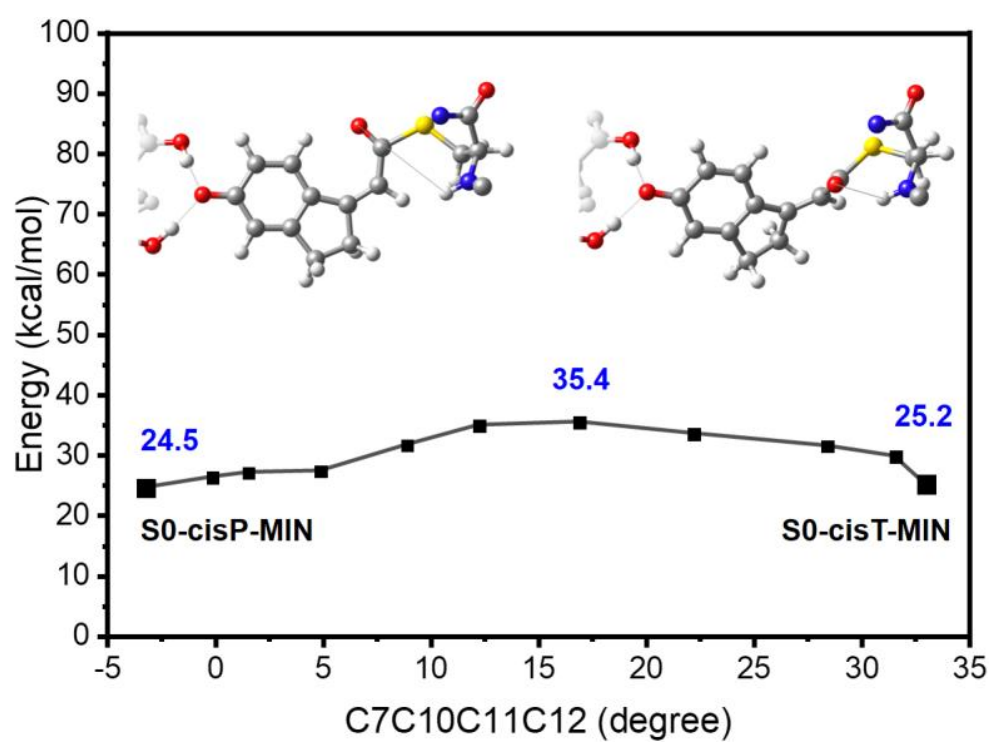

Figure S3. QM(CASPT2//CASSCF)/MM calculated minimum-energy reaction path between S0cisP-MIN and S0-cisT-MIN of the single-bond-rotation locked PYP chromophore in the wild-type protein.

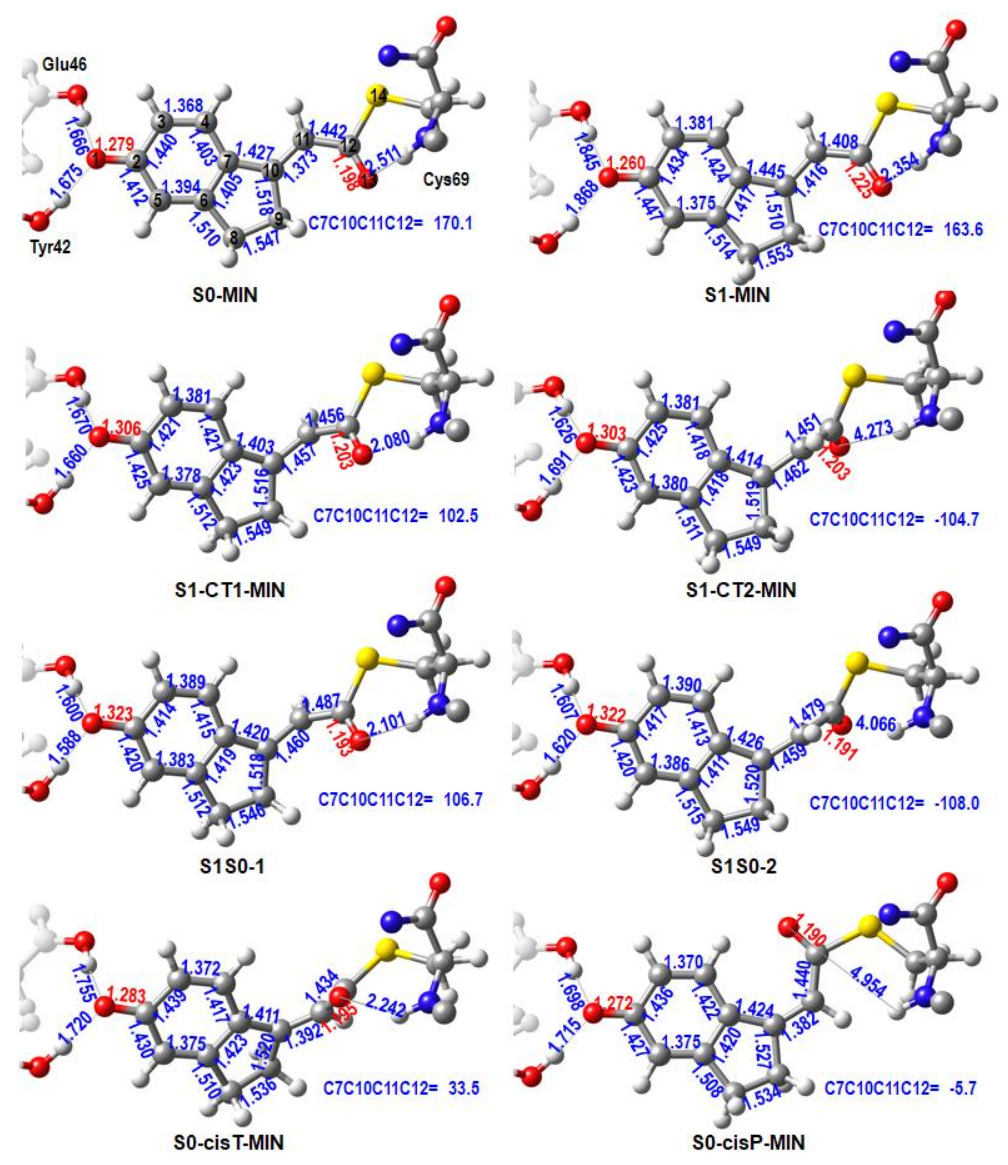

Figure S4. QM(CASSCF)/MM optimized minimum-energy structures and conical intersections in and between the $S_{0}$ and $S_{1}$ states of the single-bond-rotation locked PYP chromophore in the R52A mutation protein. Also shown are selected geometric parameters (in angstrom and degree). 


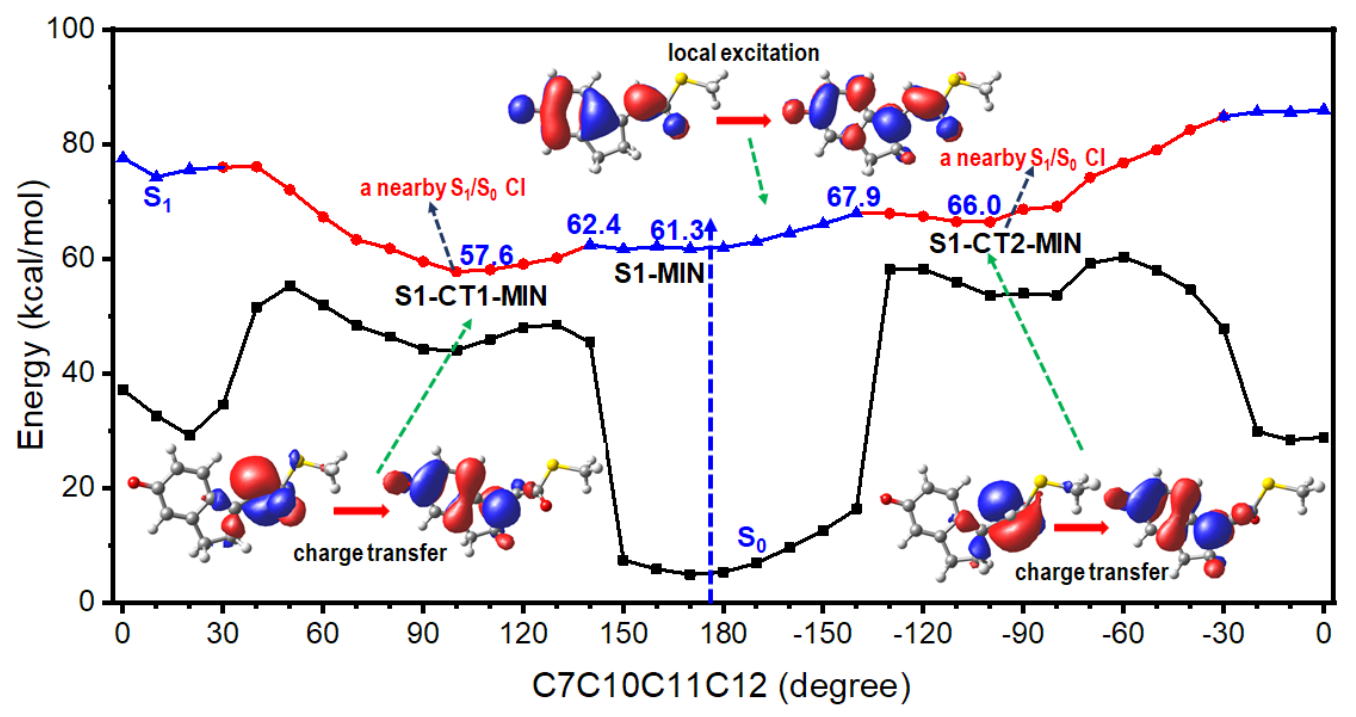

Figure S5. QM(CASPT2//CASSCF)/MM calculated minimum-energy isomerization path of the single-bond-rotation locked PYP chromophore in the R52A mutated protein along the rotation of the $\mathrm{C} 10-\mathrm{C} 11$ bond ( $\mathrm{S}_{1}$ : relaxed and $\mathrm{S}_{0}$ : unrelaxed). Also shown are energies of some key stationary points and conical intersections, and relevant molecular orbitals. 

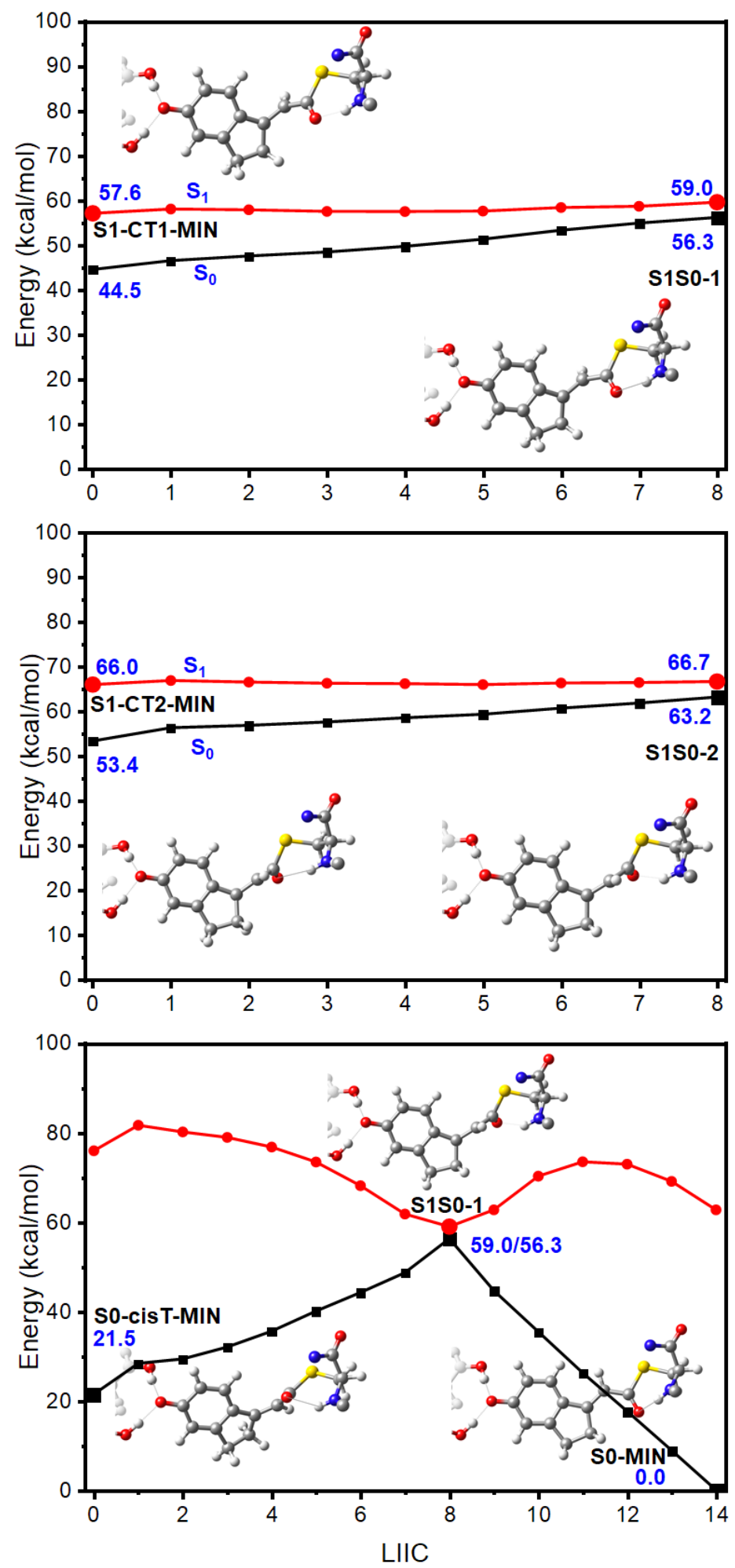

Figure S6. QM(CASPT2//CASSCF)/MM calculated linearly interpolated internal coordinate (LIIC) paths connecting both (top) S1-CT1-MIN and S1S0-1 or (middle) S1-CT2-MIN and S1S0-2 or (bottom) S0-cisT-MIN and S0-MIN via S1S0-1 of the PYP chromophore in the R52A mutation protein. 


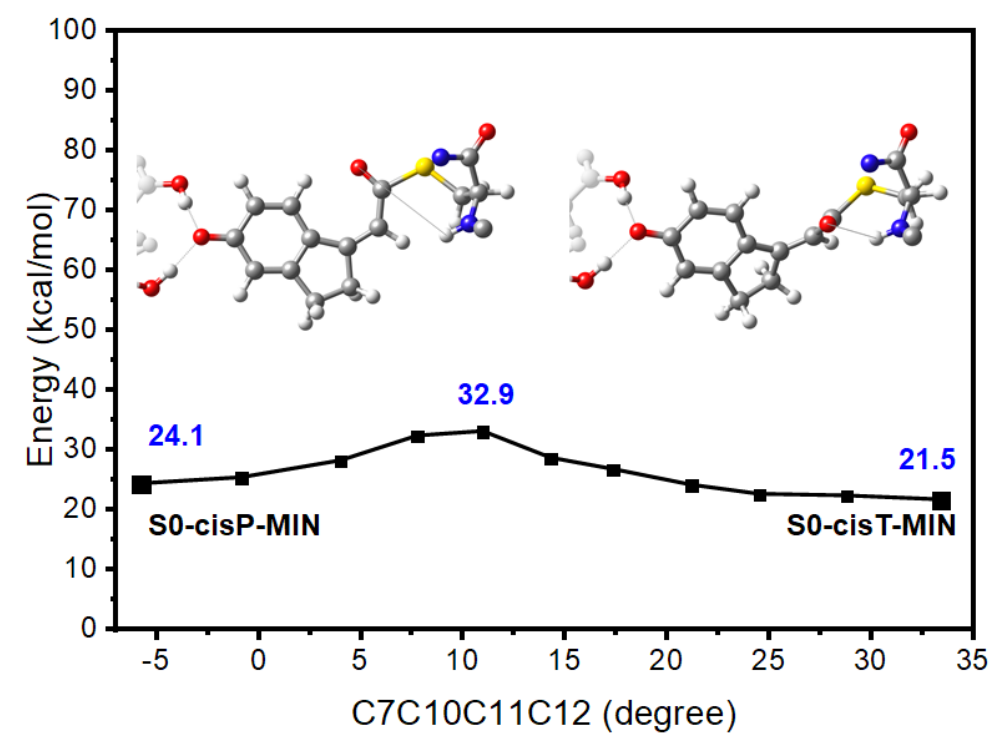

Figure S7. QM(CASPT2//CASSCF)/MM calculated minimum energy reaction path between S0cisP-MIN and S0-cisT-MIN of the single-bond-rotation locked PYP chromophore in the R52A mutation protein.

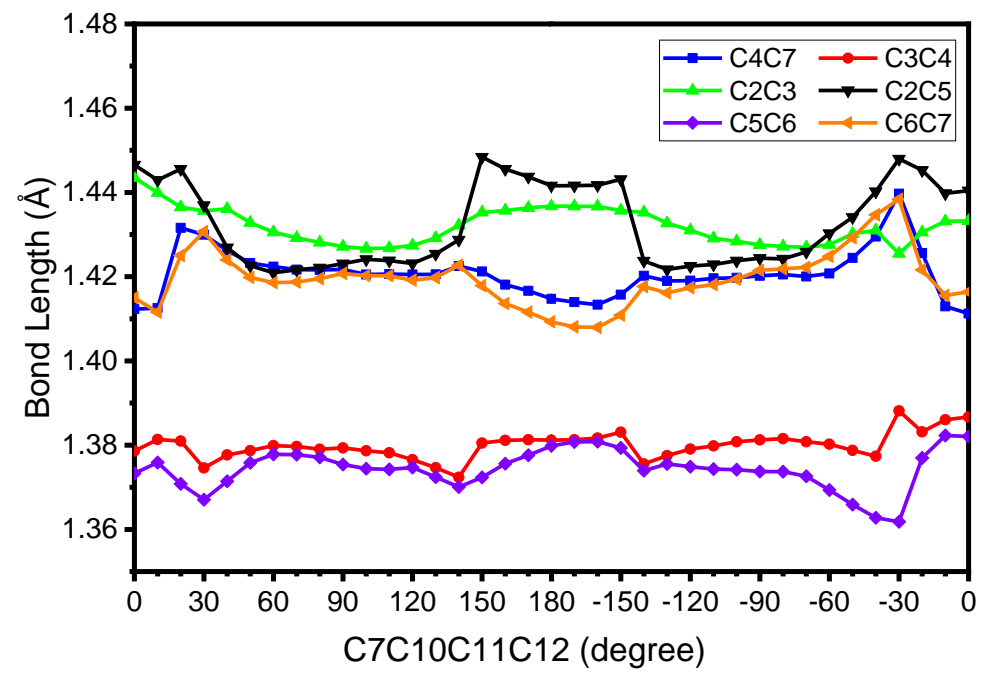

Figure S8. Variation of selected bond lengths (in angstrom) along the rotation of the C7-C10C11-C12 dihedral angle in the $S_{1}$ potential energy surface of the single-bond-rotation locked PYP chromophore in the wild-type protein. 


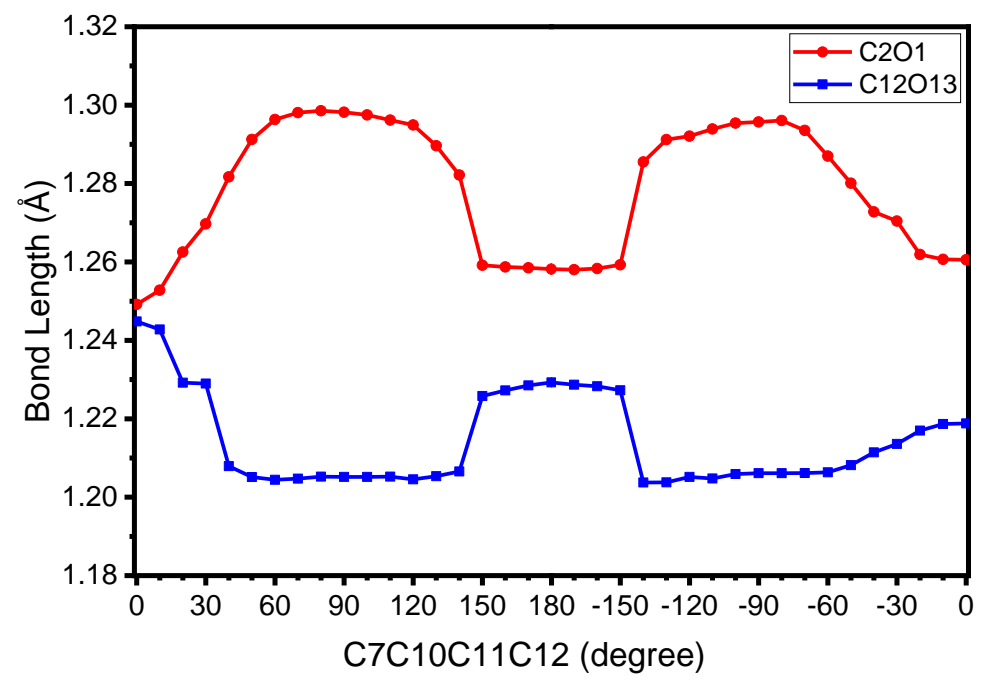

Figure S9. Variation of carbonyl bond lengths (in angstrom) along the rotation of the $\mathrm{C} 7-\mathrm{C} 10-$ C11-C12 dihedral angle in the $S_{1}$ potential energy surface of the single-bond-rotation locked PYP chromophore in the wild-type protein. 

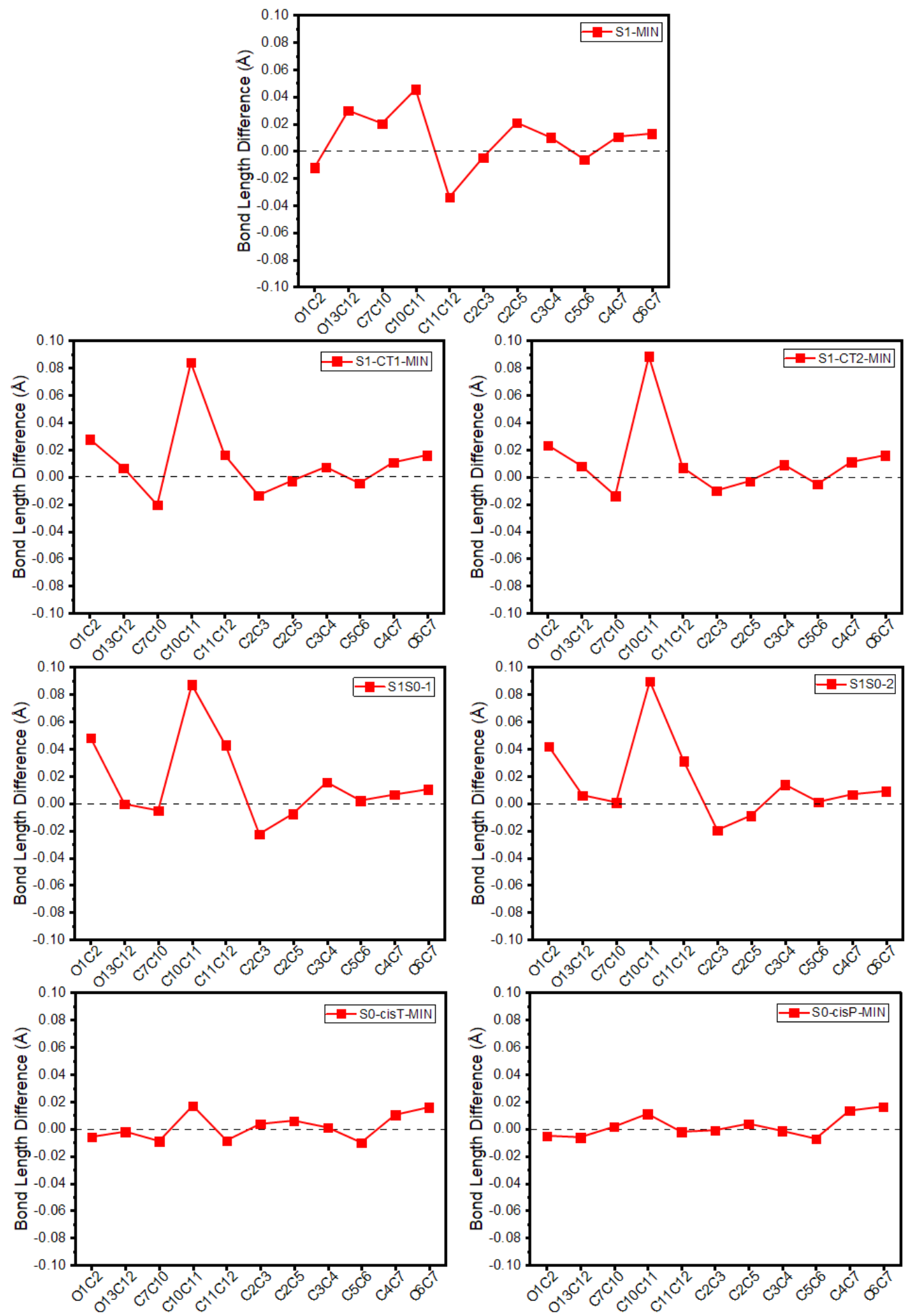

Figure S10. Selected bond-length differences of excited-states minima and conical intersections relative to those of S0-MIN for the single-bond-rotation locked PYP chromophore in the wild-type protein. 

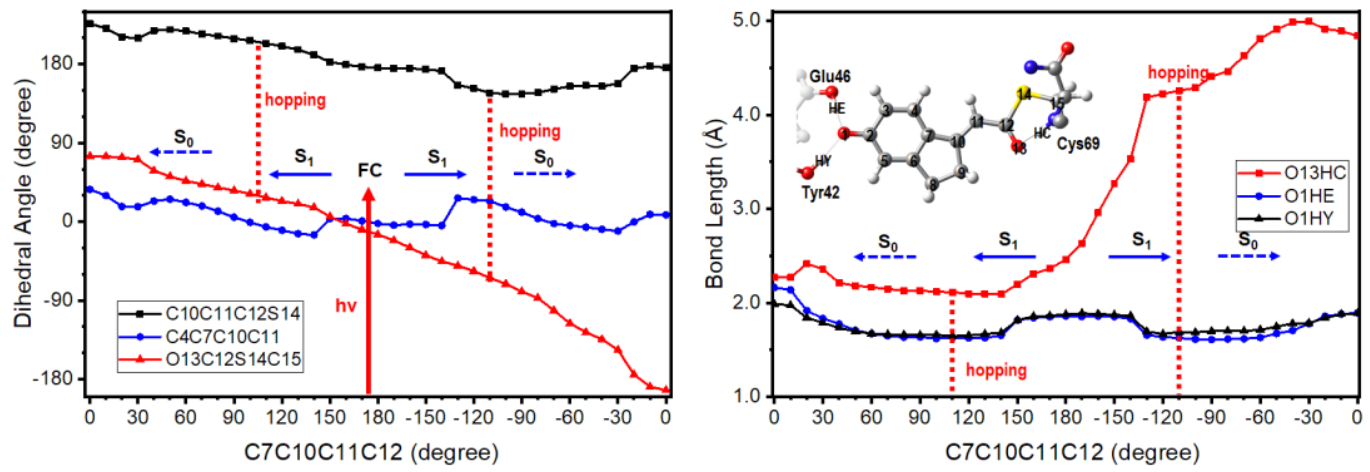

Figure S11. Variation of (left) selected dihedral angles (in degree) and (right) hydrogen-bonding distances (in angstrom) along the minimum-energy photoisomerization pathway along the rotation of the $\mathrm{C} 7-\mathrm{C} 10-\mathrm{C} 11-\mathrm{C} 12$ dihedral angle of the single-bond-rotation locked PYP chromophore in the R52A mutation protein.

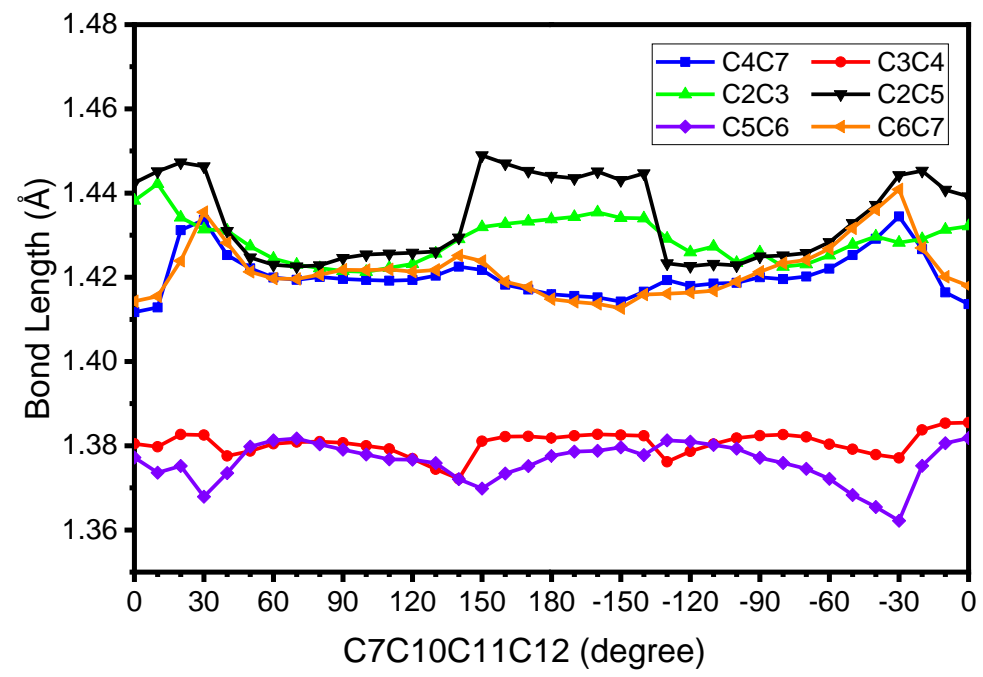

Figure S12. Variation of selected bond lengths (in angstrom) along the rotation of the C7-C10C11-C12 dihedral angle in the $S_{1}$ potential energy surface of the single-bond-rotation locked PYP chromophore in the R52A mutation protein. 


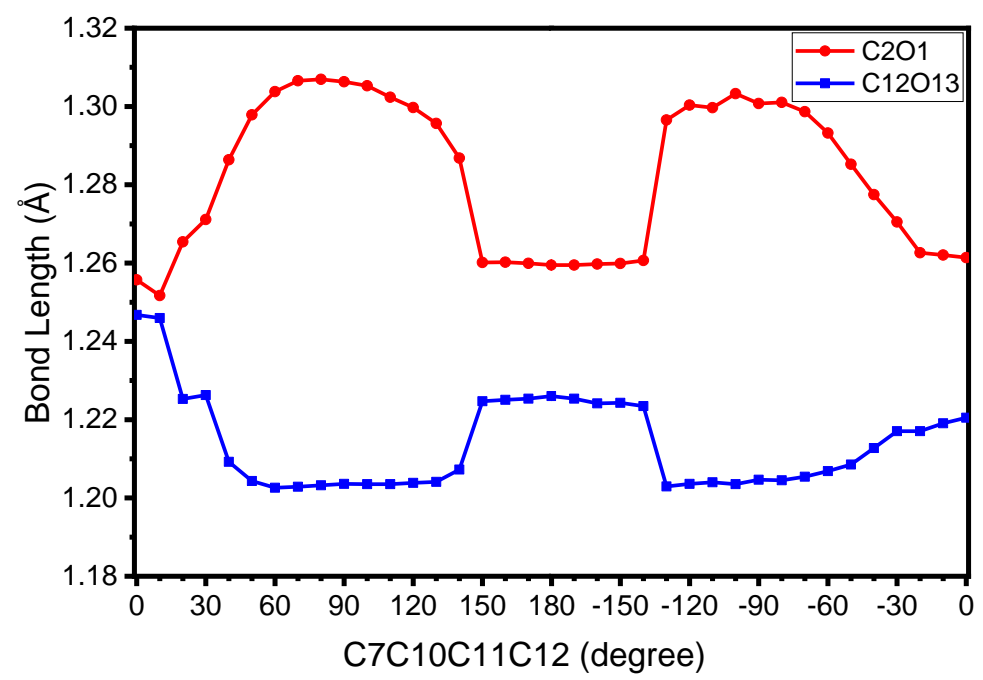

Figure S13. Variation of carbonyl bond lengths (in angstrom) along the rotation of the $\mathrm{C} 7-\mathrm{C} 10$ C11-C12 dihedral angle in the $S_{1}$ potential energy surface of the single-bond-rotation locked PYP chromophore in the R52A mutation protein. 

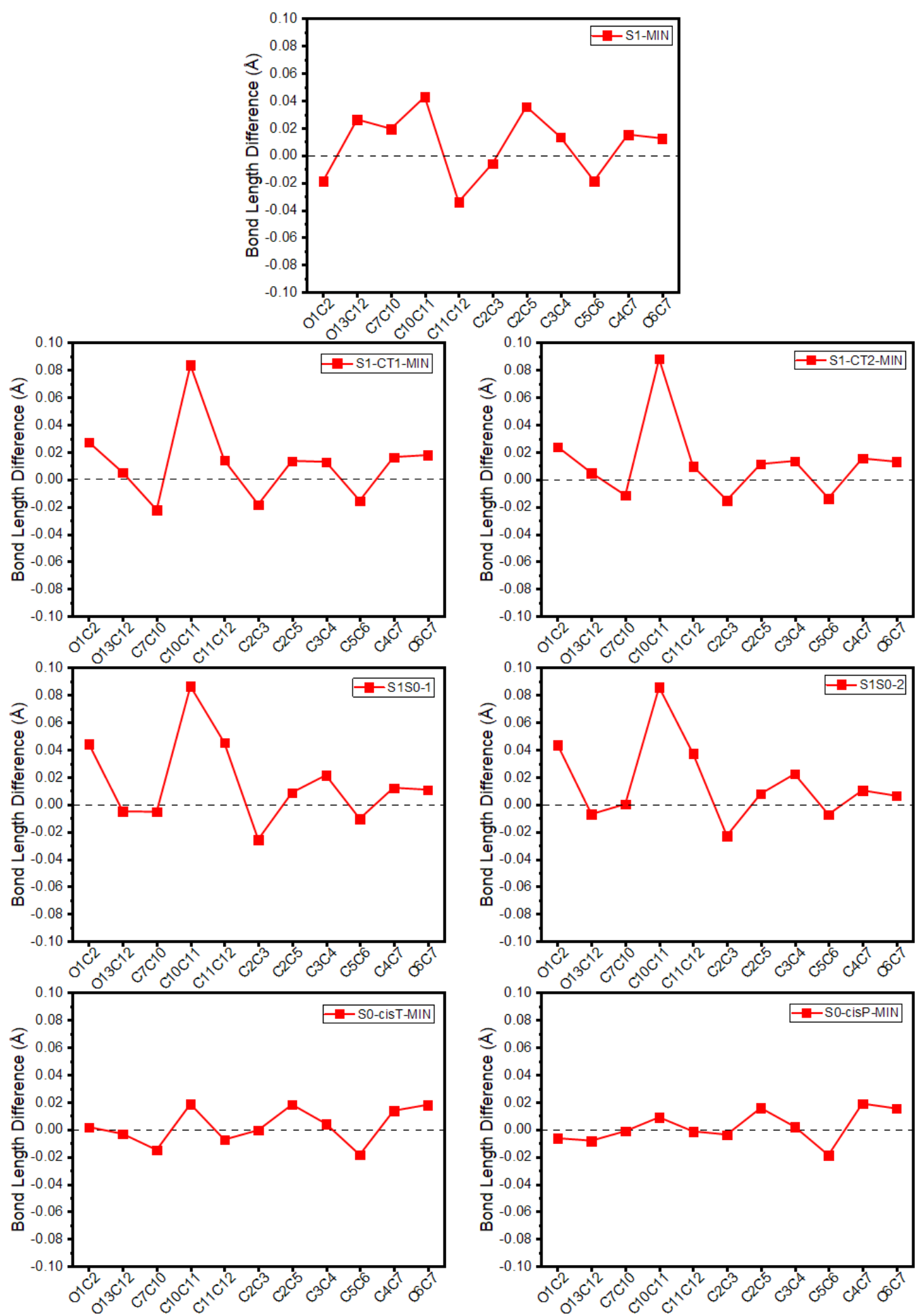

Figure S14. Selected bond-length differences of excited-states minima and conical intersections relative to those of S0-MIN for the single-bond-rotation locked PYP chromophore in the R52A mutation protein. 


\section{References}

(1) Pande, K.; Hutchison, C. D.; Groenhof, G.; Aquila, A.; Robinson, J. S.; Tenboer, J.; Basu, S.; Boutet, S.; DePonte, D. P.; Liang, M. Femtosecond Structural Dynamics Drives the Trans/Cis Isomerization in Photoactive Yellow Protein. Science 2016, 352, 725-729.

(2) Wang, J.; Wolf, R. M.; Caldwell, J. W.; Kollman, P. A.; Case, D. A. Development and Testing of a General Amber Force Field. J. Comput. Chem. 2004, 25, 1157-1174.

(3) Jorgensen, W. L.; Chandrasekhar, J.; Madura, J. D.; Impey, R. W.; Klein, M. L. Comparison of Simple Potential Functions for Simulating Liquid Water. J. Chem. Phys. 1983, 79, 926935.

(4) Case, D.; Ben-Shalom, I.; Brozell, S.; Cerutti, D.; Cheatham III, T.; Cruzeiro, V.; Darden, T.; Duke, R.; Ghoreishi, D.; Gilson, M. Amber 18; 2018. University of California, San Francisco.

(5) Warshel, A.; Levitt, M. Theoretical Studies of Enzymic Reactions: Dielectric, Electrostatic and Steric Stabilization of the Carbonium Ion in the Reaction of Lysozyme. J. Mol. Biol. 1976, 103, 227-249.

(6) Senn, H. M.; Thiel, W. Qm/Mm Methods for Biomolecular Systems. Angew. Chem. Int. Ed. 2009, 48, 1198-1229.

(7) Field, M. J.; Bash, P. A.; Karplus, M. A Combined Quantum Mechanical and Molecular Mechanical Potential for Molecular Dynamics Simulations. J. Comput. Chem. 1990, 11, 700-733.

(8) Maseras, F.; Morokuma, K. Imomm: A New Integrated Ab Initio+ Molecular Mechanics Geometry Optimization Scheme of Equilibrium Structures and Transition States. J. Comput. Chem. 1995, 16, 1170-1179.

(9) De Vries, A. H.; Sherwood, P.; Collins, S. J.; Rigby, A. M.; Rigutto, M.; Kramer, G. J. Zeolite Structure and Reactivity by Combined Quantum-Chemical-Classical Calculations. J. Phys. Chem. B 1999, 103, 6133-6141. 
(10) Roos, B. O. The Complete Active Space Self-Consistent Field Method and Its Applications in Electronic Structure Calculations. Adv. Chem. Phys. 1987, 69, 399-445.

(11) Andersson, K.; Malmqvist, P. A.; Roos, B. O.; Sadlej, A. J.; Wolinski, K. Second-Order Perturbation Theory with a Casscf Reference Function. J. Phys. Chem. 1990, 94, 54835488.

(12) Andersson, K.; Malmqvist, P. Å.; Roos, B. O. Second-Order Perturbation Theory with a Complete Active Space Self-Consistent Field Reference Function. J. Chem. Phys. 1992, $96,1218-1226$.

(13) Roos, B. O.; Lindh, R.; Malmqvist, P.-Å.; Veryazov, V.; Widmark, P.-O. Main Group Atoms and Dimers Studied with a New Relativistic Ano Basis Set. J. Phys. Chem. A 2004, 108, $2851-2858$

(14) Roos, B. O.; Veryazov, V.; Widmark, P.-O. Relativistic Atomic Natural Orbital Type Basis Sets for the Alkaline and Alkaline-Earth Atoms Applied to the Ground-State Potentials for the Corresponding Dimers. Theor. Chem. Acc. 2004, 111, 345-351.

(15) Aquilante, F.; Lindh, R.; Bondo Pedersen, T. Unbiased Auxiliary Basis Sets for Accurate Two-Electron Integral Approximations. J. Chem. Phys. 2007, 127, 114107.

(16) Aquilante, F.; Pedersen, T. B.; Lindh, R.; Roos, B. O.; Sánchez de Merás, A.; Koch, H. Accurate Ab Initio Density Fitting for Multiconfigurational Self-Consistent Field Methods. J. Chem. Phys. 2008, 129, 024113.

(17) Forsberg, N.; Malmqvist, P.-Å. Multiconfiguration Perturbation Theory with Imaginary Level Shift. Chem. Phys. Lett. 1997, 274, 196-204.

(18) Ghigo, G.; Roos, B. O.; Malmqvist, P.-Å. A Modified Definition of the Zeroth-Order Hamiltonian in Multiconfigurational Perturbation Theory (CASPT2). Chem. Phys. Lett. 2004, 396, 142-149. 
(19) Zobel, J. P.; Nogueira, J. J.; González, L. The Ipea Dilemma in Caspt2. Chem. Sci. 2017, 8, 1482-1499.

(20) Fdez. Galván, I.; Vacher, M.; Alavi, A.; Angeli, C.; Aquilante, F.; Autschbach, J.; Bao, J. J.; Bokarev, S. I.; Bogdanov, N. A.; Carlson, R. K. Openmolcas: From Source Code to Insight. J. Chem. Theory Comput. 2019, 15, 5925-5964.

(21) Ponder, J. W. Tinker: Software Tools for Molecular Design. Version: 2004. 


\section{Cartesian coordinates of all optimized structures (QM region)}

The QM(CASSCF)/MM optimized minimum-energy structures and conical intersections in and between the $S_{0}$ and $S_{1}$ states of the single-bond-rotation locked PYP chromophore in the wild-type protein.

$\begin{array}{llll}\text { SO-MIN } & & & \\ \text { C } & 44.800361 & 39.830241 & 35.780655 \\ \text { C } & 45.152321 & 39.127146 & 34.638331 \\ \text { H } & 46.107224 & 38.641081 & 34.575051 \\ \text { C } & 44.300894 & 39.063182 & 33.553676 \\ \text { H } & 44.590435 & 38.543555 & 32.658938 \\ \text { C } & 43.070788 & 39.707151 & 33.593280 \\ \text { O } & 42.283181 & 39.646109 & 32.520550 \\ \text { H } & 41.355720 & 39.861625 & 32.730675 \\ \text { C } & 42.702811 & 40.397433 & 34.743595 \\ \text { H } & 41.743929 & 40.879207 & 34.773377 \\ \text { C } & 43.566535 & 40.462610 & 35.818714 \\ \text { H } & 43.267457 & 41.022202 & 36.687782 \\ \text { C } & 41.773670 & 35.922801 & 34.630458 \\ \text { H } & 41.569536 & 35.559656 & 35.630840 \\ \text { H } & 42.808796 & 36.244629 & 34.610803 \\ \text { C } & 40.868427 & 37.108428 & 34.330316 \\ \text { H } & 41.139039 & 37.939526 & 34.975340 \\ \text { H } & 40.989327 & 37.485559 & 33.321728 \\ \text { C } & 39.400906 & 36.860275 & 34.576022 \\ \text { O } & 38.973052 & 35.855773 & 35.052117 \\ \text { O } & 38.605692 & 37.842658 & 34.241345 \\ \text { H } & 39.073608 & 38.620261 & 33.885746 \\ \text { N } & 32.492103 & 46.945091 & 29.357798 \\ \text { H } & 33.291279 & 46.427527 & 29.084051 \\ \text { C } & 31.263218 & 46.251869 & 29.543489 \\ \text { C } & 31.304269 & 44.847967 & 28.925217 \\ \text { S } & 32.146516 & 43.564312 & 29.917208 \\ \text { C } & 33.831509 & 43.458934 & 29.218338 \\ \text { C } & 34.636184 & 42.569244 & 30.019058 \\ \text { C } & 35.964684 & 42.288454 & 29.826215 \\ \text { C } & 36.795486 & 41.613478 & 30.762330 \\ \text { C } & 36.473376 & 41.079880 & 32.026881 \\ \text { C } & 37.463309 & 40.543482 & 32.808897 \\ \text { O } & 38.845923 & 40.528589 & 32.407256 \\ & 39.727246 & 40.011532 & 33.162118\end{array}$




$\begin{array}{llll}\text { C } & 39.138591 & 41.042237 & 31.109100 \\ \text { C } & 38.130879 & 41.568386 & 30.327199 \\ \text { C } & 38.233166 & 42.145246 & 28.935850 \\ \text { C } & 36.804390 & 42.638629 & 28.610980 \\ \text { H } & 36.776345 & 43.697903 & 28.394055 \\ \text { H } & 36.393016 & 42.137379 & 27.740855 \\ \text { H } & 38.550500 & 41.377518 & 28.237543 \\ \text { H } & 38.957721 & 42.952181 & 28.881795 \\ \text { H } & 40.159939 & 41.021341 & 30.769532 \\ \text { H } & 37.223077 & 40.131817 & 33.771822 \\ \text { H } & 35.458951 & 41.078518 & 32.380298 \\ \text { H } & 34.164220 & 42.198050 & 30.907382 \\ \text { O } & 34.132094 & 44.058573 & 28.225854 \\ \text { H } & 30.291384 & 44.487245 & 28.832347 \\ \text { H } & 31.755589 & 44.890450 & 27.944239 \\ \text { H } & 30.433874 & 46.765889 & 29.064379 \\ \text { C } & 30.876887 & 46.164370 & 31.014338 \\ \text { O } & 29.757418 & 45.968691 & 31.369055 \\ \text { H } & 32.598626 & 47.898806 & 29.554127 \\ \text { H } & 31.669303 & 46.297384 & 31.696351 \\ \text { H } & 45.526600 & 39.981716 & 36.592336 \\ \text { H } & 41.642513 & 35.079465 & 33.917787\end{array}$

$\begin{array}{llll}\text { SO-cisT-MIN } & & & \\ \text { C } & 44.683785 & 39.884976 & 35.788394 \\ \text { C } & 44.964101 & 39.208393 & 34.607604 \\ \text { H } & 45.902971 & 38.700677 & 34.475332 \\ \text { C } & 44.054440 & 39.192162 & 33.570250 \\ \text { H } & 44.285419 & 38.695588 & 32.645777 \\ \text { C } & 42.844952 & 39.869870 & 33.684378 \\ \text { O } & 42.026373 & 39.870071 & 32.630730 \\ \text { H } & 41.122216 & 40.154416 & 32.823111 \\ \text { C } & 42.549745 & 40.532871 & 34.868281 \\ \text { H } & 41.616128 & 41.057369 & 34.955352 \\ \text { C } & 43.468288 & 40.542439 & 35.903098 \\ \text { H } & 43.229910 & 41.091197 & 36.797195 \\ \text { C } & 41.402346 & 35.940038 & 34.446524 \\ \text { H } & 41.043488 & 35.597770 & 35.411625 \\ \text { H } & 42.417720 & 36.298565 & 34.585536 \\ \text { C } & 40.536738 & 37.108785 & 33.959604 \\ \text { H } & 40.658178 & 37.940453 & 34.647732 \\ \text { H } & 40.872542 & 37.496216 & 33.002042 \\ \text { C } & 39.048802 & 36.854724 & 33.846154 \\ \text { O } & 38.538023 & 35.788110 & 33.968259\end{array}$




\begin{tabular}{|c|c|c|c|}
\hline 0 & 38.314062 & 37.914525 & 33.593328 \\
\hline $\mathrm{H}$ & 38.790400 & 38.745513 & 33.472326 \\
\hline $\mathrm{N}$ & 32.594167 & 46.764954 & 29.439198 \\
\hline $\mathrm{H}$ & 33.442405 & 46.251582 & 29.431834 \\
\hline C & 31.381236 & 46.046431 & 29.612763 \\
\hline C & 31.307309 & 44.742037 & 28.811198 \\
\hline$S$ & 32.011264 & 43.166653 & 29.444910 \\
\hline C & 33.844513 & 43.327676 & 29.529420 \\
\hline C & 34.534148 & 42.272886 & 28.845902 \\
\hline C & 35.838258 & 41.844165 & 29.053660 \\
\hline C & 36.658763 & 41.775591 & 30.201859 \\
\hline C & 36.326568 & 41.778640 & 31.582409 \\
\hline C & 37.241893 & 41.343807 & 32.506932 \\
\hline C & 38.572980 & 40.909799 & 32.156129 \\
\hline 0 & 39.356165 & 40.420269 & 33.019638 \\
\hline C & 38.910270 & 40.975234 & 30.765575 \\
\hline C & 37.971211 & 41.362492 & 29.846921 \\
\hline$C$ & 38.079684 & 41.347759 & 28.346735 \\
\hline C & 36.613159 & 41.190915 & 27.919126 \\
\hline $\mathrm{H}$ & 36.397145 & 41.616985 & 26.947937 \\
\hline $\mathrm{H}$ & 36.366783 & 40.135233 & 27.878399 \\
\hline $\mathrm{H}$ & 38.717118 & 40.558923 & 27.969355 \\
\hline $\mathrm{H}$ & 38.467589 & 42.297896 & 27.985745 \\
\hline $\mathrm{H}$ & 39.888237 & 40.642712 & 30.462002 \\
\hline $\mathrm{H}$ & 36.966129 & 41.266801 & 33.541438 \\
\hline $\mathrm{H}$ & 35.335880 & 42.045796 & 31.901898 \\
\hline $\mathrm{H}$ & 34.014888 & 41.894163 & 27.986579 \\
\hline 0 & 34.308216 & 44.255899 & 30.123765 \\
\hline $\mathrm{H}$ & 30.266729 & 44.460365 & 28.727319 \\
\hline $\mathrm{H}$ & 31.689092 & 44.914407 & 27.816591 \\
\hline $\mathrm{H}$ & 30.556914 & 46.651898 & 29.241396 \\
\hline C & 31.025163 & 45.850123 & 31.078303 \\
\hline 0 & 29.929950 & 45.537859 & 31.422925 \\
\hline $\mathrm{H}$ & 32.642493 & 47.726555 & 29.609487 \\
\hline H & 31.790826 & 46.063797 & 31.771253 \\
\hline $\mathrm{H}$ & 45.459314 & 40.014972 & 36.556952 \\
\hline $\mathrm{H}$ & 41.423103 & 35.071004 & 33.751810 \\
\hline
\end{tabular}

S0-cisP-MIN

$\begin{array}{llll}\text { C } & 44.770882 & 39.871865 & 35.799768 \\ \text { C } & 45.101825 & 39.179605 & 34.642744 \\ \text { H } & 46.045121 & 38.675667 & 34.550693 \\ \text { C } & 44.226693 & 39.130756 & 33.577305 \\ \text { H } & 44.497676 & 38.618198 & 32.673612\end{array}$




\begin{tabular}{|c|c|c|c|}
\hline C & 43.004939 & 39.789672 & 33.641275 \\
\hline 0 & 42.210501 & 39.753439 & 32.570457 \\
\hline $\mathrm{H}$ & 41.298576 & 40.017997 & 32.760675 \\
\hline$C$ & 42.658953 & 40.466013 & 34.805124 \\
\hline $\mathrm{H}$ & 41.709575 & 40.965137 & 34.860318 \\
\hline$C$ & 43.541803 & 40.511987 & 35.866373 \\
\hline $\mathrm{H}$ & 43.259612 & 41.066850 & 36.744145 \\
\hline C & 41.675978 & 35.966925 & 34.580268 \\
\hline $\mathrm{H}$ & 41.439207 & 35.607792 & 35.575199 \\
\hline $\mathrm{H}$ & 42.699394 & 36.324184 & 34.595471 \\
\hline C & 40.756332 & 37.129987 & 34.213350 \\
\hline $\mathrm{H}$ & 40.966766 & 37.969725 & 34.869241 \\
\hline $\mathrm{H}$ & 40.953302 & 37.510349 & 33.216686 \\
\hline C & 39.274801 & 36.855482 & 34.318197 \\
\hline 0 & 38.816348 & 35.816180 & 34.673222 \\
\hline 0 & 38.492431 & 37.855971 & 33.990719 \\
\hline $\mathrm{H}$ & 38.955882 & 38.663820 & 33.723630 \\
\hline $\mathrm{N}$ & 32.608797 & 46.940500 & 29.539957 \\
\hline $\mathrm{H}$ & 33.471689 & 46.455968 & 29.478073 \\
\hline C & 31.425435 & 46.161574 & 29.742087 \\
\hline C & 31.622277 & 44.723687 & 29.180463 \\
\hline$S$ & 31.788307 & 43.376829 & 30.418070 \\
\hline C & 33.462875 & 42.625073 & 30.555777 \\
\hline C & 34.508113 & 42.884281 & 29.599701 \\
\hline C & 35.828572 & 42.487024 & 29.697836 \\
\hline C & 36.621563 & 41.809268 & 30.666363 \\
\hline C & 36.299435 & 41.104110 & 31.859779 \\
\hline C & 37.292208 & 40.588800 & 32.649481 \\
\hline C & 38.691842 & 40.723328 & 32.344809 \\
\hline 0 & 39.574119 & 40.202020 & 33.086776 \\
\hline C & 39.002947 & 41.417451 & 31.133694 \\
\hline C & 38.001783 & 41.934368 & 30.350050 \\
\hline C & 38.180780 & 42.728916 & 29.082467 \\
\hline C & 36.753062 & 42.858759 & 28.541622 \\
\hline $\mathrm{H}$ & 36.538306 & 43.849788 & 28.155824 \\
\hline $\mathrm{H}$ & 36.585268 & 42.147411 & 27.736487 \\
\hline $\mathrm{H}$ & 38.846890 & 42.236237 & 28.385574 \\
\hline $\mathrm{H}$ & 38.605766 & 43.705617 & 29.292078 \\
\hline $\mathrm{H}$ & 40.038785 & 41.547956 & 30.869371 \\
\hline $\mathrm{H}$ & 37.030775 & 40.056849 & 33.545533 \\
\hline $\mathrm{H}$ & 35.282154 & 40.936774 & 32.131560 \\
\hline $\mathrm{H}$ & 34.235648 & 43.474473 & 28.746087 \\
\hline 0 & 33.479999 & 41.886469 & 31.490767 \\
\hline $\mathrm{H}$ & 30.741720 & 44.444843 & 28.620548 \\
\hline
\end{tabular}




$\begin{array}{llll}\mathrm{H} & 32.451421 & 44.724353 & 28.488223 \\ \mathrm{H} & 30.590265 & 46.609482 & 29.212515 \\ \mathrm{C} & 31.013014 & 46.162008 & 31.214570 \\ \mathrm{O} & 29.882509 & 46.073116 & 31.568251 \\ \mathrm{H} & 32.646999 & 47.907229 & 29.691220 \\ \mathrm{H} & 31.807892 & 46.293276 & 31.894728 \\ \mathrm{H} & 45.513091 & 40.018490 & 36.598486 \\ \mathrm{H} & 41.605848 & 35.112929 & 33.870877\end{array}$

$\begin{array}{llll}\text { S1-MIN } & & & \\ \text { C } & 44.846385 & 39.792648 & 35.786422 \\ \text { C } & 45.203297 & 39.016742 & 34.693981 \\ \text { H } & 46.132332 & 38.482588 & 34.684730 \\ \text { C } & 44.375135 & 38.913869 & 33.595471 \\ \text { H } & 44.660029 & 38.326136 & 32.743424 \\ \text { C } & 43.171149 & 39.599545 & 33.572707 \\ \text { O } & 42.411760 & 39.498596 & 32.475667 \\ \text { H } & 41.508025 & 39.770049 & 32.634176 \\ \text { C } & 42.793275 & 40.363509 & 34.668224 \\ \text { H } & 41.852840 & 40.881995 & 34.647458 \\ \text { C } & 43.633611 & 40.462114 & 35.760866 \\ \text { H } & 43.333644 & 41.077125 & 36.591080 \\ \text { C } & 41.725590 & 35.933006 & 34.613411 \\ \text { H } & 41.538345 & 35.557530 & 35.612286 \\ \text { H } & 42.754072 & 36.274765 & 34.590847 \\ \text { C } & 40.793111 & 37.101909 & 34.326518 \\ \text { H } & 41.095695 & 37.956311 & 34.926568 \\ \text { H } & 40.849849 & 37.444198 & 33.299078 \\ \text { C } & 39.348188 & 36.846766 & 34.667451 \\ \text { O } & 38.958159 & 35.876950 & 35.226771 \\ \text { O } & 38.515386 & 37.805575 & 34.319645 \\ \text { H } & 38.966479 & 38.548014 & 33.916701 \\ \text { N } & 32.469028 & 46.893909 & 29.289989 \\ \text { H } & 33.245056 & 46.334477 & 29.025637 \\ \text { C } & 31.230911 & 46.246052 & 29.540439 \\ \text { C } & 31.210006 & 44.827516 & 28.961871 \\ \text { S } & 32.064006 & 43.524654 & 29.906166 \\ \text { C } & 33.797566 & 43.556477 & 29.275858 \\ \text { C } & 34.602215 & 42.647765 & 29.989527 \\ \text { C } & 35.968701 & 42.367363 & 29.741419 \\ \text { C } & 36.806234 & 41.701747 & 30.708639 \\ \text { C } & 36.468125 & 41.185460 & 31.987838 \\ \text { C } & 37.455326 & 40.620457 & 32.770533 \\ \text { C } & 38.824454 & 40.572441 & 32.343586\end{array}$




\begin{tabular}{|c|c|c|c|}
\hline 0 & 39.710557 & 40.025343 & 33.049607 \\
\hline C & 39.142758 & 41.110161 & 31.038228 \\
\hline C & 38.150614 & 41.655210 & 30.260424 \\
\hline C & 38.245544 & 42.260929 & 28.879247 \\
\hline C & 36.792150 & 42.659409 & 28.510983 \\
\hline $\mathrm{H}$ & 36.716897 & 43.693302 & 28.199659 \\
\hline $\mathrm{H}$ & 36.433216 & 42.059759 & 27.675370 \\
\hline $\mathrm{H}$ & 38.651258 & 41.541799 & 28.176771 \\
\hline $\mathrm{H}$ & 38.908948 & 43.120697 & 28.871835 \\
\hline $\mathrm{H}$ & 40.164930 & 41.051602 & 30.707779 \\
\hline $\mathrm{H}$ & 37.219144 & 40.209577 & 33.734744 \\
\hline $\mathrm{H}$ & 35.455611 & 41.207968 & 32.337163 \\
\hline $\mathrm{H}$ & 34.153717 & 42.181291 & 30.844437 \\
\hline 0 & 34.089081 & 44.285638 & 28.332362 \\
\hline $\mathrm{H}$ & 30.180644 & 44.502768 & 28.926753 \\
\hline $\mathrm{H}$ & 31.608560 & 44.845807 & 27.957652 \\
\hline $\mathrm{H}$ & 30.401031 & 46.764427 & 29.065572 \\
\hline C & 30.827330 & 46.211374 & 31.011594 \\
\hline 0 & 29.696219 & 46.107171 & 31.357512 \\
\hline $\mathrm{H}$ & 32.618172 & 47.839544 & 29.495055 \\
\hline $\mathrm{H}$ & 31.612484 & 46.308234 & 31.708090 \\
\hline $\mathrm{H}$ & 45.554025 & 39.962325 & 36.610458 \\
\hline $\mathrm{H}$ & 41.610736 & 35.092308 & 33.895279 \\
\hline \multicolumn{4}{|c|}{ S1-CT1-MIN } \\
\hline C & 44.738746 & 39.874358 & 35.873612 \\
\hline C & 45.015485 & 39.129874 & 34.733492 \\
\hline $\mathrm{H}$ & 45.934126 & 38.582606 & 34.634363 \\
\hline C & 44.103015 & 39.082666 & 33.702530 \\
\hline $\mathrm{H}$ & 44.321919 & 38.518115 & 32.817552 \\
\hline C & 42.900136 & 39.776224 & 33.792358 \\
\hline 0 & 42.067156 & 39.733511 & 32.761412 \\
\hline $\mathrm{H}$ & 41.145047 & 39.977738 & 32.985935 \\
\hline C & 42.614447 & 40.501833 & 34.945107 \\
\hline $\mathrm{H}$ & 41.680415 & 41.025283 & 35.014015 \\
\hline C & 43.535493 & 40.558344 & 35.969431 \\
\hline $\mathrm{H}$ & 43.303041 & 41.149170 & 36.838286 \\
\hline C & 41.433053 & 35.903215 & 34.491781 \\
\hline $\mathrm{H}$ & 41.110947 & 35.507216 & 35.447911 \\
\hline $\mathrm{H}$ & 42.447733 & 36.261955 & 34.624340 \\
\hline C & 40.542566 & 37.074503 & 34.077346 \\
\hline $\mathrm{H}$ & 40.695220 & 37.894026 & 34.773620 \\
\hline $\mathrm{H}$ & 40.829763 & 37.492767 & 33.117099 \\
\hline C & 39.055170 & 36.814508 & 34.020852 \\
\hline
\end{tabular}




\begin{tabular}{|c|c|c|c|}
\hline 0 & 38.562593 & 35.733559 & 34.145733 \\
\hline 0 & 38.311691 & 37.871116 & 33.825675 \\
\hline $\mathrm{H}$ & 38.815100 & 38.704072 & 33.690798 \\
\hline $\mathrm{N}$ & 32.579140 & 46.811578 & 29.345631 \\
\hline $\mathrm{H}$ & 33.408652 & 46.290541 & 29.192888 \\
\hline C & 31.396900 & 46.089077 & 29.625537 \\
\hline C & 31.416934 & 44.686207 & 29.005931 \\
\hline S & 32.307928 & 43.328662 & 29.854124 \\
\hline C & 34.012252 & 43.463179 & 29.278800 \\
\hline C & 34.728624 & 42.193357 & 29.248168 \\
\hline C & 36.175525 & 42.078973 & 29.356399 \\
\hline C & 36.880590 & 41.717170 & 30.512067 \\
\hline C & 36.433228 & 41.407792 & 31.824142 \\
\hline C & 37.338685 & 40.929166 & 32.746078 \\
\hline C & 38.722278 & 40.736136 & 32.458975 \\
\hline 0 & 39.516172 & 40.179302 & 33.321845 \\
\hline C & 39.159308 & 41.093361 & 31.152134 \\
\hline C & 38.263204 & 41.563881 & 30.221470 \\
\hline C & 38.513220 & 41.882418 & 28.767078 \\
\hline C & 37.107274 & 42.111988 & 28.163213 \\
\hline $\mathrm{H}$ & 37.056361 & 43.069738 & 27.652555 \\
\hline $\mathrm{H}$ & 36.845461 & 41.346420 & 27.433331 \\
\hline $\mathrm{H}$ & 39.024637 & 41.063156 & 28.274628 \\
\hline $\mathrm{H}$ & 39.133374 & 42.768180 & 28.653231 \\
\hline $\mathrm{H}$ & 40.191878 & 40.929855 & 30.894454 \\
\hline $\mathrm{H}$ & 36.994604 & 40.642578 & 33.723099 \\
\hline $\mathrm{H}$ & 35.395595 & 41.495467 & 32.088038 \\
\hline $\mathrm{H}$ & 34.129386 & 41.322246 & 29.053544 \\
\hline 0 & 34.498824 & 44.512366 & 28.942380 \\
\hline $\mathrm{H}$ & 30.404858 & 44.306181 & 28.985407 \\
\hline $\mathrm{H}$ & 31.782275 & 44.767862 & 27.991072 \\
\hline $\mathrm{H}$ & 30.537160 & 46.585413 & 29.185779 \\
\hline C & 30.990709 & 46.028236 & 31.098380 \\
\hline 0 & 29.875749 & 45.820066 & 31.440053 \\
\hline $\mathrm{H}$ & 32.670088 & 47.760534 & 29.564536 \\
\hline $\mathrm{H}$ & 31.753887 & 46.218788 & 31.800433 \\
\hline $\mathrm{H}$ & 45.501801 & 40.004879 & 36.654769 \\
\hline $\mathrm{H}$ & 41.450591 & 35.065067 & 33.761290 \\
\hline \multicolumn{4}{|c|}{ S1-CT2-MIN } \\
\hline C & 44.747654 & 39.894867 & 35.844901 \\
\hline C & 45.036030 & 39.199975 & 34.678881 \\
\hline $\mathrm{H}$ & 45.969629 & 38.681190 & 34.566321 \\
\hline C & 44.127489 & 39.156874 & 33.640044 \\
\hline
\end{tabular}




\begin{tabular}{|c|c|c|c|}
\hline $\mathrm{H}$ & 44.355528 & 38.628353 & 32.733425 \\
\hline C & 42.910532 & 39.819319 & 33.745876 \\
\hline 0 & 42.082693 & 39.788132 & 32.706101 \\
\hline $\mathrm{H}$ & 41.157940 & 40.006999 & 32.926212 \\
\hline C & 42.607879 & 40.503999 & 34.918378 \\
\hline $\mathrm{H}$ & 41.658744 & 41.000312 & 34.994658 \\
\hline C & 43.528077 & 40.547711 & 35.946712 \\
\hline $\mathrm{H}$ & 43.286562 & 41.103298 & 36.835585 \\
\hline C & 41.562987 & 35.974775 & 34.534615 \\
\hline $\mathrm{H}$ & 41.305224 & 35.600308 & 35.519516 \\
\hline $\mathrm{H}$ & 42.587394 & 36.329799 & 34.596506 \\
\hline C & 40.645982 & 37.138910 & 34.160685 \\
\hline $\mathrm{H}$ & 40.853958 & 37.971648 & 34.827347 \\
\hline $\mathrm{H}$ & 40.848162 & 37.526726 & 33.167138 \\
\hline C & 39.163175 & 36.863081 & 34.256202 \\
\hline 0 & 38.709187 & 35.799795 & 34.548262 \\
\hline 0 & 38.385621 & 37.878294 & 33.996068 \\
\hline $\mathrm{H}$ & 38.851308 & 38.705873 & 33.748488 \\
\hline N & 32.629403 & 46.742595 & 29.347001 \\
\hline $\mathrm{H}$ & 33.458218 & 46.201583 & 29.324924 \\
\hline C & 31.412716 & 46.064180 & 29.615712 \\
\hline C & 31.412340 & 44.617736 & 29.096720 \\
\hline S & 32.402005 & 43.442478 & 30.117569 \\
\hline C & 33.714038 & 42.846097 & 28.996049 \\
\hline C & 35.044124 & 43.203892 & 29.446239 \\
\hline C & 36.301428 & 42.472317 & 29.320596 \\
\hline C & 36.925483 & 41.812211 & 30.396579 \\
\hline C & 36.426791 & 41.378395 & 31.654125 \\
\hline C & 37.295149 & 40.828074 & 32.574409 \\
\hline C & 38.700485 & 40.698071 & 32.346060 \\
\hline 0 & 39.477006 & 40.160124 & 33.229858 \\
\hline C & 39.175077 & 41.106617 & 31.067899 \\
\hline C & 38.312566 & 41.640049 & 30.140507 \\
\hline C & 38.621125 & 42.090561 & 28.736488 \\
\hline C & 37.342108 & 42.820520 & 28.271274 \\
\hline $\mathrm{H}$ & 37.495110 & 43.898110 & 28.213301 \\
\hline $\mathrm{H}$ & 37.038512 & 42.498945 & 27.280115 \\
\hline $\mathrm{H}$ & 38.801649 & 41.221164 & 28.111370 \\
\hline $\mathrm{H}$ & 39.501416 & 42.722061 & 28.677146 \\
\hline $\mathrm{H}$ & 40.220294 & 40.980431 & 30.843575 \\
\hline $\mathrm{H}$ & 36.919607 & 40.491903 & 33.524439 \\
\hline $\mathrm{H}$ & 35.378916 & 41.447275 & 31.882479 \\
\hline $\mathrm{H}$ & 35.064040 & 44.166148 & 29.922856 \\
\hline 0 & 33.441866 & 42.200064 & 28.015013 \\
\hline
\end{tabular}




$\begin{array}{rrrr}\text { H } & 30.401866 & 44.241705 & 29.125999 \\ \text { H } & 31.770327 & 44.595891 & 28.076592 \\ \text { H } & 30.583332 & 46.550889 & 29.112556 \\ \mathrm{C} & 31.000889 & 46.118578 & 31.084470 \\ \mathrm{O} & 29.867450 & 46.041168 & 31.426393 \\ \mathrm{H} & 32.72895 & 47.70585 & 29.500112 \\ \mathrm{H} & 31.785122 & 46.259533 & 31.780472 \\ \mathrm{H} & 45.513512 & 40.035921 & 36.622179 \\ \mathrm{H} & 41.523863 & 35.124706 & 33.820301\end{array}$

$\begin{array}{llll}\text { S1S0-1 } & & & \\ \text { C } & 44.745486 & 39.885341 & 35.865809 \\ \text { C } & 45.008995 & 39.133527 & 34.728745 \\ \text { H } & 45.928144 & 38.585322 & 34.627749 \\ \text { C } & 44.088788 & 39.075682 & 33.703743 \\ \text { H } & 44.295431 & 38.498576 & 32.822487 \\ \text { C } & 42.884191 & 39.769399 & 33.790009 \\ \text { O } & 42.044100 & 39.714556 & 32.768175 \\ \text { H } & 41.108537 & 39.946925 & 33.001019 \\ \text { C } & 42.612730 & 40.507355 & 34.939358 \\ \text { H } & 41.680181 & 41.034130 & 35.008570 \\ \text { C } & 43.541047 & 40.568538 & 35.957965 \\ \text { H } & 43.314661 & 41.166286 & 36.824026 \\ \text { C } & 41.466398 & 35.920145 & 34.529645 \\ \text { H } & 41.148226 & 35.522309 & 35.486654 \\ \text { H } & 42.481324 & 36.284782 & 34.650432 \\ \text { C } & 40.574258 & 37.092158 & 34.128244 \\ \text { H } & 40.730820 & 37.907893 & 34.827101 \\ \text { H } & 40.855671 & 37.513642 & 33.169628 \\ \text { C } & 39.085447 & 36.834021 & 34.088049 \\ \text { O } & 38.598300 & 35.753798 & 34.250776 \\ \text { O } & 38.348418 & 37.884075 & 33.867668 \\ \text { H } & 38.857387 & 38.725255 & 33.709520 \\ \text { N } & 32.567721 & 46.882072 & 29.323138 \\ \text { H } & 33.398863 & 46.364545 & 29.171227 \\ \text { C } & 31.382661 & 46.155658 & 29.622323 \\ \text { C } & 31.404693 & 44.755867 & 28.996893 \\ \text { S } & 32.287184 & 43.394862 & 29.845411 \\ \text { C } & 33.972438 & 43.522765 & 29.245446 \\ \text { C } & 34.733776 & 42.248211 & 29.277237 \\ \text { C } & 36.184352 & 42.172811 & 29.410234 \\ \text { C } & 36.888310 & 41.753377 & 30.565864 \\ \text { C } & 36.464287 & 41.469635 & 31.886871 \\ \text { C } & 37.375935 & 40.957075 & 32.796766\end{array}$




$\begin{array}{llll}\text { C } & 38.735383 & 40.706726 & 32.484244 \\ \text { O } & 39.544267 & 40.121317 & 33.344843 \\ \text { C } & 39.152468 & 41.041425 & 31.170195 \\ \text { C } & 38.250292 & 41.536157 & 30.247981 \\ \text { C } & 38.496102 & 41.850650 & 28.792033 \\ \text { C } & 37.088245 & 42.083596 & 28.197104 \\ \text { H } & 37.057310 & 42.992037 & 27.603242 \\ \text { H } & 36.793254 & 41.264511 & 27.539367 \\ \text { H } & 39.004629 & 41.034816 & 28.292157 \\ \text { H } & 39.113058 & 42.738839 & 28.677154 \\ \text { H } & 40.172690 & 40.844368 & 30.887302 \\ \text { H } & 37.038232 & 40.691402 & 33.782716 \\ \text { H } & 35.441517 & 41.610509 & 32.188407 \\ \text { H } & 34.150918 & 41.375764 & 29.036056 \\ \text { O } & 34.444169 & 44.535072 & 28.814008 \\ \text { H } & 30.392707 & 44.378773 & 28.960038 \\ \text { H } & 31.779338 & 44.830738 & 27.985688 \\ \text { H } & 30.527871 & 46.648509 & 29.167504 \\ \text { C } & 30.993451 & 46.086549 & 31.102082 \\ \text { O } & 29.872945 & 45.903975 & 31.440104 \\ \text { H } & 32.662590 & 47.829223 & 29.550203 \\ \text { H } & 31.763433 & 46.244523 & 31.800061 \\ \text { H } & 45.509500 & 40.010895 & 36.646730 \\ \text { H } & 41.476475 & 35.087300 & 33.792447\end{array}$

\section{S1S0-2}

$\begin{array}{llll}\text { C } & 44.736467 & 39.896416 & 35.839590 \\ \mathrm{C} & 45.022293 & 39.161202 & 34.695456 \\ \mathrm{H} & 45.946964 & 38.624877 & 34.593855 \\ \mathrm{C} & 44.111226 & 39.108517 & 33.662928 \\ \mathrm{H} & 44.338452 & 38.548741 & 32.777070 \\ \mathrm{C} & 42.896707 & 39.784643 & 33.750989 \\ \mathrm{O} & 42.062527 & 39.731711 & 32.723909 \\ \mathrm{H} & 41.120585 & 39.936682 & 32.947611 \\ \mathrm{C} & 42.605470 & 40.503845 & 34.908493 \\ \mathrm{H} & 41.663935 & 41.013695 & 34.978890 \\ \mathrm{C} & 43.524086 & 40.566473 & 35.934660 \\ \mathrm{H} & 43.283630 & 41.151976 & 36.805229 \\ \mathrm{C} & 41.509329 & 35.949959 & 34.524825 \\ \mathrm{H} & 41.222504 & 35.551765 & 35.490718 \\ \mathrm{H} & 42.528604 & 36.306181 & 34.620880 \\ \mathrm{C} & 40.600137 & 37.116822 & 34.143454 \\ \mathrm{H} & 40.775450 & 37.938357 & 34.832921 \\ \mathrm{H} & 40.845449 & 37.537004 & 33.174331\end{array}$




\begin{tabular}{llll} 
C & 39.112376 & 36.854293 & 34.156924 \\
O & 38.641491 & 35.783071 & 34.405801 \\
O & 38.359989 & 37.881639 & 33.887480 \\
H & 38.860426 & 38.717023 & 33.670666 \\
N & 32.633996 & 46.737027 & 29.326804 \\
H & 33.464439 & 46.194603 & 29.302727 \\
C & 31.420988 & 46.060749 & 29.616009 \\
C & 31.414835 & 44.614582 & 29.098108 \\
S & 32.351451 & 43.429759 & 30.149863 \\
C & 33.693851 & 42.863360 & 29.059544 \\
C & 35.043859 & 43.227838 & 29.523952 \\
C & 36.283590 & 42.465981 & 29.390766 \\
C & 36.937067 & 41.842557 & 30.490013 \\
C & 36.462050 & 41.427138 & 31.757975 \\
C & 37.347583 & 40.860021 & 32.658782 \\
C & 38.729522 & 40.679641 & 32.386185 \\
O & 39.526567 & 40.093240 & 33.247881 \\
C & 39.187709 & 41.098210 & 31.111975 \\
C & 38.310325 & 41.659989 & 30.205861 \\
C & 38.602619 & 42.104084 & 28.795953 \\
C & 37.319151 & 42.832841 & 28.341438 \\
H & 37.473101 & 43.910980 & 28.296233 \\
H & 37.024224 & 42.514423 & 27.345835 \\
H & 38.773028 & 41.237840 & 28.164328 \\
H & 39.483508 & 42.732284 & 28.721055 \\
H & 40.223885 & 40.954416 & 30.859976 \\
H & 36.982040 & 40.532233 & 33.615093 \\
H & 35.428003 & 41.526399 & 32.031090 \\
H & 35.068135 & 44.213289 & 29.949532 \\
O & 33.467010 & 42.248286 & 28.049949 \\
H & 30.402753 & 44.241348 & 29.085785 \\
H & 31.812604 & 44.595491 & 28.091652 \\
H & 30.600618 & 46.553037 & 29.106149 \\
& 30.993680 & 46.110824 & 31.084293 \\
H & 29.858555 & 46.023244 & 31.416288 \\
H & 32.739792 & 47.697932 & 29.49031 \\
H & 45.496904 & 46.252813 & 31.788818 \\
H & 40.032306 & 36.623351 \\
H & & 35.112491 & 33.793488 \\
\hline
\end{tabular}

The QM(CASSCF)/MM optimized minimum-energy isomerization path along the rotation of the $\mathrm{C} 10$ $\mathrm{C}_{11}$ bond in the $\mathrm{S}_{1}$ state of the single-bond-rotation locked PYP chromophore in the wild-type protein 


\begin{tabular}{|c|c|c|c|}
\hline \multicolumn{4}{|c|}{ C7-C10-C11-C12 $0^{\circ}$} \\
\hline C & 44.671242 & 39.869270 & 35.798657 \\
\hline C & 44.941762 & 39.112601 & 34.664820 \\
\hline $\mathrm{H}$ & 45.865753 & 38.574552 & 34.562405 \\
\hline C & 44.027364 & 39.051199 & 33.635202 \\
\hline $\mathrm{H}$ & 44.248597 & 38.489818 & 32.747948 \\
\hline C & 42.836280 & 39.761072 & 33.715385 \\
\hline 0 & 42.007962 & 39.709979 & 32.662211 \\
\hline $\mathrm{H}$ & 41.158470 & 40.111379 & 32.830909 \\
\hline C & 42.549602 & 40.495891 & 34.855404 \\
\hline $\mathrm{H}$ & 41.627690 & 41.042939 & 34.920689 \\
\hline C & 43.468924 & 40.554793 & 35.885165 \\
\hline $\mathrm{H}$ & 43.231466 & 41.155681 & 36.745563 \\
\hline C & 41.339253 & 35.896208 & 34.444007 \\
\hline $\mathrm{H}$ & 41.001186 & 35.512071 & 35.398812 \\
\hline $\mathrm{H}$ & 42.333074 & 36.304306 & 34.589275 \\
\hline C & 40.415673 & 37.028631 & 33.987822 \\
\hline $\mathrm{H}$ & 40.485061 & 37.842574 & 34.705114 \\
\hline $\mathrm{H}$ & 40.736579 & 37.465970 & 33.047388 \\
\hline C & 38.947055 & 36.700744 & 33.847505 \\
\hline 0 & 38.476929 & 35.616589 & 33.938893 \\
\hline 0 & 38.164202 & 37.740482 & 33.599551 \\
\hline $\mathrm{H}$ & 38.639602 & 38.562032 & 33.510437 \\
\hline N & 32.705282 & 46.618470 & 29.387944 \\
\hline $\mathrm{H}$ & 33.544378 & 46.081854 & 29.445235 \\
\hline C & 31.467002 & 45.954097 & 29.622054 \\
\hline C & 31.316350 & 44.615490 & 28.903610 \\
\hline S & 32.076832 & 43.132267 & 29.657367 \\
\hline C & 33.838676 & 43.389406 & 29.509816 \\
\hline C & 34.558324 & 42.511226 & 28.573414 \\
\hline C & 35.696508 & 41.823863 & 28.837722 \\
\hline C & 36.501831 & 41.763373 & 30.083846 \\
\hline C & 36.099225 & 41.726383 & 31.437039 \\
\hline C & 37.009744 & 41.352677 & 32.402367 \\
\hline C & 38.375751 & 41.012892 & 32.082709 \\
\hline 0 & 39.163355 & 40.567101 & 32.943739 \\
\hline C & 38.773432 & 41.127954 & 30.696672 \\
\hline C & 37.842989 & 41.465834 & 29.744788 \\
\hline C & 37.981060 & 41.423657 & 28.241013 \\
\hline C & 36.542312 & 41.101476 & 27.807018 \\
\hline $\mathrm{H}$ & 36.316914 & 41.366664 & 26.782152 \\
\hline $\mathrm{H}$ & 36.396114 & 40.030591 & 27.912737 \\
\hline $\mathrm{H}$ & 38.696319 & 40.681436 & 27.910876 \\
\hline $\mathrm{H}$ & 38.289901 & 42.393694 & 27.855299 \\
\hline
\end{tabular}




$\begin{array}{llll}\mathrm{H} & 39.783335 & 40.856913 & 30.442078 \\ \mathrm{H} & 36.713961 & 41.265897 & 33.430051 \\ \mathrm{H} & 35.082177 & 41.940857 & 31.697405 \\ \mathrm{H} & 34.126804 & 42.387095 & 27.590331 \\ \mathrm{O} & 34.434100 & 44.201821 & 30.241261 \\ \mathrm{H} & 30.264357 & 44.362738 & 28.878326 \\ \mathrm{H} & 31.668920 & 44.729631 & 27.886863 \\ \mathrm{H} & 30.671086 & 46.578931 & 29.216217 \\ \mathrm{C} & 31.085598 & 45.849833 & 31.094519 \\ \mathrm{O} & 29.960508 & 45.635511 & 31.424768 \\ \mathrm{H} & 32.759677 & 47.583251 & 29.569069 \\ \mathrm{H} & 31.846717 & 46.037857 & 31.796285 \\ \mathrm{H} & 45.442379 & 40.016679 & 36.569411 \\ \mathrm{H} & 41.405487 & 35.045715 & 33.728946\end{array}$

$\begin{array}{lrll}\text { C7-C10-C11-C12 10 } & & \\ \text { C } & 44.655294 & 39.886199 & 35.813876 \\ \text { C } & 44.912161 & 39.119883 & 34.683884 \\ \text { H } & 45.830227 & 38.569924 & 34.586042 \\ \text { C } & 43.995097 & 39.060615 & 33.655092 \\ \text { H } & 44.202718 & 38.482751 & 32.774745 \\ \text { C } & 42.812037 & 39.783891 & 33.731944 \\ \text { O } & 41.976855 & 39.729990 & 32.686034 \\ \text { H } & 41.122198 & 40.119761 & 32.859216 \\ \text { C } & 42.541004 & 40.535553 & 34.864737 \\ \text { H } & 41.625380 & 41.095014 & 34.922152 \\ \mathrm{C} & 43.464101 & 40.589865 & 35.892683 \\ \text { H } & 43.241120 & 41.199248 & 36.750436 \\ \text { C } & 41.326033 & 35.900344 & 34.445730 \\ \text { H } & 40.980259 & 35.519679 & 35.399814 \\ \text { H } & 42.324206 & 36.296056 & 34.601088 \\ \text { C } & 40.411759 & 37.041414 & 33.984366 \\ \text { H } & 40.491521 & 37.861111 & 34.694067 \\ \text { H } & 40.732326 & 37.466690 & 33.037862 \\ \text { C } & 38.941114 & 36.718625 & 33.850989 \\ \text { O } & 38.470677 & 35.634442 & 33.941072 \\ \text { O } & 38.155231 & 37.755204 & 33.612731 \\ \text { H } & 38.609418 & 38.587276 & 33.512870 \\ \text { N } & 32.725611 & 46.598670 & 29.365843 \\ \text { H } & 33.550072 & 46.047436 & 29.456539 \\ \text { C } & 31.479213 & 45.958500 & 29.614025 \\ \text { C } & 31.313084 & 44.628965 & 28.872526 \\ \text { S } & 32.039063 & 43.105014 & 29.586200 \\ \text { C } & 33.824228 & 43.323752 & 29.513409\end{array}$




\begin{tabular}{llll} 
C & 34.558473 & 42.433260 & 28.609120 \\
C & 35.763629 & 41.852686 & 28.857406 \\
C & 36.547820 & 41.784334 & 30.121603 \\
C & 36.124561 & 41.717019 & 31.467559 \\
C & 37.024785 & 41.332946 & 32.442352 \\
C & 38.392675 & 41.000660 & 32.139116 \\
O & 39.176953 & 40.544363 & 33.002962 \\
C & 38.808181 & 41.137609 & 30.764104 \\
C & 37.892719 & 41.498215 & 29.802346 \\
C & 38.064029 & 41.483009 & 28.303225 \\
C & 36.637120 & 41.173984 & 27.820063 \\
H & 36.449322 & 41.496499 & 26.803659 \\
H & 36.490733 & 40.097548 & 27.862052 \\
H & 38.782881 & 40.742484 & 27.975953 \\
H & 38.388453 & 42.457351 & 27.941547 \\
H & 39.821906 & 40.875487 & 30.515862 \\
H & 36.710790 & 41.233612 & 33.463708 \\
H & 35.104472 & 41.920567 & 31.723575 \\
H & 34.114647 & 42.256395 & 27.640543 \\
O & 34.396011 & 44.123234 & 30.273937 \\
H & 30.258122 & 44.390431 & 28.843021 \\
H & 31.660998 & 44.762464 & 27.856579 \\
H & 30.682529 & 46.588677 & 29.223255 \\
C & 31.089806 & 45.857229 & 31.091059 \\
O & 29.964944 & 45.657268 & 31.425301 \\
H & 32.802747 & 47.566563 & 29.542566 \\
H & 31.849001 & 46.040653 & 31.801439 \\
H & 45.426661 & 40.021881 & 36.587424 \\
H & 41.388182 & 35.048817 & 33.731974 \\
& & & \\
\hline
\end{tabular}

$\begin{array}{lrrr}\text { C7-C10-C11-C12 20 } & & \\ \mathrm{C} & 44.631150 & 39.955602 & 35.854712 \\ \mathrm{C} & 44.867131 & 39.205847 & 34.708983 \\ \mathrm{H} & 45.785173 & 38.657644 & 34.586538 \\ \mathrm{C} & 43.932341 & 39.167756 & 33.694837 \\ \mathrm{H} & 44.122981 & 38.605555 & 32.800349 \\ \mathrm{C} & 42.748769 & 39.889842 & 33.800705 \\ \mathrm{O} & 41.899970 & 39.854639 & 32.768887 \\ \mathrm{H} & 41.017800 & 40.182346 & 32.966626 \\ \mathrm{C} & 42.501917 & 40.625445 & 34.950627 \\ \mathrm{H} & 41.588220 & 41.184465 & 35.032922 \\ \mathrm{C} & 43.442608 & 40.660567 & 35.963144 \\ \mathrm{H} & 43.238008 & 41.259709 & 36.833118 \\ \mathrm{C} & 41.360330 & 35.951608 & 34.494522\end{array}$




\begin{tabular}{|c|c|c|c|}
\hline $\mathrm{H}$ & 41.014478 & 35.582235 & 35.453351 \\
\hline $\mathrm{H}$ & 42.364008 & 36.336881 & 34.640273 \\
\hline C & 40.459106 & 37.101132 & 34.028007 \\
\hline $\mathrm{H}$ & 40.561393 & 37.929030 & 34.723981 \\
\hline $\mathrm{H}$ & 40.775065 & 37.506199 & 33.071349 \\
\hline C & 38.980370 & 36.806653 & 33.914368 \\
\hline 0 & 38.499472 & 35.725428 & 34.018468 \\
\hline 0 & 38.209373 & 37.848533 & 33.674629 \\
\hline $\mathrm{H}$ & 38.662694 & 38.688933 & 33.578504 \\
\hline $\mathrm{N}$ & 32.699330 & 46.674526 & 29.394493 \\
\hline $\mathrm{H}$ & 33.536247 & 46.140862 & 29.435059 \\
\hline C & 31.471245 & 46.003298 & 29.643543 \\
\hline C & 31.363345 & 44.655388 & 28.924820 \\
\hline S & 32.030367 & 43.146479 & 29.715705 \\
\hline C & 33.848405 & 43.301563 & 29.652016 \\
\hline C & 34.541519 & 42.454257 & 28.746550 \\
\hline C & 35.888405 & 41.933880 & 28.949825 \\
\hline C & 36.611192 & 41.815752 & 30.172398 \\
\hline C & 36.158796 & 41.731359 & 31.528004 \\
\hline C & 37.031637 & 41.314522 & 32.513657 \\
\hline C & 38.401145 & 40.968901 & 32.251765 \\
\hline 0 & 39.168249 & 40.488052 & 33.131691 \\
\hline C & 38.850017 & 41.128320 & 30.886953 \\
\hline C & 37.972178 & 41.493420 & 29.899409 \\
\hline C & 38.216527 & 41.486446 & 28.412365 \\
\hline C & 36.880604 & 41.941703 & 27.799106 \\
\hline $\mathrm{H}$ & 36.973771 & 42.956332 & 27.418520 \\
\hline $\mathrm{H}$ & 36.568400 & 41.319399 & 26.964458 \\
\hline $\mathrm{H}$ & 38.466057 & 40.485990 & 28.074247 \\
\hline $\mathrm{H}$ & 39.037566 & 42.140992 & 28.140667 \\
\hline $\mathrm{H}$ & 39.872583 & 40.874582 & 30.664309 \\
\hline $\mathrm{H}$ & 36.685266 & 41.195714 & 33.523712 \\
\hline $\mathrm{H}$ & 35.133446 & 41.928843 & 31.767975 \\
\hline $\mathrm{H}$ & 33.960926 & 41.997545 & 27.963657 \\
\hline 0 & 34.386813 & 44.117842 & 30.396825 \\
\hline $\mathrm{H}$ & 30.315555 & 44.404655 & 28.835818 \\
\hline $\mathrm{H}$ & 31.775202 & 44.749774 & 27.929602 \\
\hline $\mathrm{H}$ & 30.660628 & 46.604215 & 29.236236 \\
\hline C & 31.083877 & 45.905780 & 31.119836 \\
\hline 0 & 29.959408 & 45.705954 & 31.447325 \\
\hline $\mathrm{H}$ & 32.770083 & 47.638892 & 29.580941 \\
\hline $\mathrm{H}$ & 31.845275 & 46.081726 & 31.829942 \\
\hline $\mathrm{H}$ & 45.413032 & 40.074020 & 36.620253 \\
\hline $\mathrm{H}$ & 41.409151 & 35.093433 & 33.786988 \\
\hline
\end{tabular}




\begin{tabular}{|c|c|c|c|}
\hline \multicolumn{4}{|c|}{ C7-C10-C11-C12 30 } \\
\hline C & 44.642882 & 39.934796 & 35.839184 \\
\hline C & 44.885587 & 39.190282 & 34.691698 \\
\hline $\mathrm{H}$ & 45.804594 & 38.642461 & 34.573571 \\
\hline C & 43.956459 & 39.153614 & 33.672436 \\
\hline $\mathrm{H}$ & 44.151254 & 38.593431 & 32.777483 \\
\hline C & 42.768310 & 39.869519 & 33.775773 \\
\hline 0 & 41.923204 & 39.836259 & 32.744210 \\
\hline $\mathrm{H}$ & 41.035028 & 40.158061 & 32.947071 \\
\hline C & 42.513345 & 40.598168 & 34.929522 \\
\hline $\mathrm{H}$ & 41.594335 & 41.148520 & 35.010259 \\
\hline C & 43.450458 & 40.633875 & 35.944972 \\
\hline $\mathrm{H}$ & 43.241633 & 41.229759 & 36.816245 \\
\hline C & 41.348864 & 35.961432 & 34.477481 \\
\hline $\mathrm{H}$ & 41.002046 & 35.590530 & 35.435734 \\
\hline $\mathrm{H}$ & 42.356706 & 36.337186 & 34.621255 \\
\hline C & 40.458059 & 37.121928 & 34.019877 \\
\hline $\mathrm{H}$ & 40.577158 & 37.947855 & 34.715156 \\
\hline $\mathrm{H}$ & 40.769054 & 37.526863 & 33.061891 \\
\hline C & 38.975638 & 36.843998 & 33.922781 \\
\hline 0 & 38.487619 & 35.764900 & 34.032023 \\
\hline 0 & 38.216708 & 37.893330 & 33.695310 \\
\hline $\mathrm{H}$ & 38.681482 & 38.732233 & 33.597794 \\
\hline N & 32.698440 & 46.648967 & 29.386437 \\
\hline $\mathrm{H}$ & 33.544016 & 46.132301 & 29.445264 \\
\hline C & 31.479797 & 45.956567 & 29.646689 \\
\hline C & 31.405676 & 44.582196 & 28.965012 \\
\hline S & 32.112907 & 43.109326 & 29.795746 \\
\hline C & 33.913558 & 43.280785 & 29.614408 \\
\hline C & 34.577240 & 42.322001 & 28.794068 \\
\hline C & 35.971289 & 41.847564 & 29.018459 \\
\hline C & 36.665632 & 41.734146 & 30.228895 \\
\hline C & 36.201979 & 41.545190 & 31.568337 \\
\hline C & 37.084176 & 41.158783 & 32.549097 \\
\hline C & 38.481876 & 40.940599 & 32.304396 \\
\hline 0 & 39.259877 & 40.453464 & 33.181640 \\
\hline C & 38.948076 & 41.245807 & 30.979911 \\
\hline C & 38.068041 & 41.583001 & 29.989605 \\
\hline C & 38.348687 & 41.738855 & 28.518313 \\
\hline C & 36.987242 & 42.135808 & 27.919823 \\
\hline $\mathrm{H}$ & 36.970600 & 43.199322 & 27.679141 \\
\hline $\mathrm{H}$ & 36.766480 & 41.595692 & 27.002152 \\
\hline $\mathrm{H}$ & 38.681901 & 40.794706 & 28.098308 \\
\hline
\end{tabular}




$\begin{array}{llll}\text { H } & 39.121894 & 42.475036 & 28.322827 \\ \text { H } & 39.996805 & 41.115225 & 30.772655 \\ \text { H } & 36.727816 & 40.962288 & 33.543987 \\ \text { H } & 35.158354 & 41.650334 & 31.799081 \\ \text { H } & 33.915713 & 41.610280 & 28.328035 \\ \text { O } & 34.480486 & 44.187343 & 30.220300 \\ \text { H } & 30.366711 & 44.293418 & 28.878792 \\ \text { H } & 31.820244 & 44.665233 & 27.969167 \\ \text { H } & 30.655754 & 46.525921 & 29.221201 \\ \text { C } & 31.085314 & 45.887658 & 31.123427 \\ \text { O } & 29.962048 & 45.676621 & 31.452309 \\ \text { H } & 32.752638 & 47.616011 & 29.566585 \\ \text { H } & 31.842216 & 46.089085 & 31.830999 \\ \text { H } & 45.422278 & 40.056811 & 36.606155 \\ \text { H } & 41.387922 & 35.107168 & 33.764953\end{array}$

$\begin{array}{llll}\text { C7-C10-C11-C12 40 } & & \\ \mathrm{C} & 44.671754 & 39.953949 & 35.846466 \\ \mathrm{C} & 44.940142 & 39.246835 & 34.680875 \\ \mathrm{H} & 45.869160 & 38.719924 & 34.553834 \\ \mathrm{C} & 44.020674 & 39.219345 & 33.653178 \\ \mathrm{H} & 44.239129 & 38.693248 & 32.743020 \\ \mathrm{C} & 42.815600 & 39.907116 & 33.763420 \\ \mathrm{O} & 41.980220 & 39.884026 & 32.727864 \\ \mathrm{H} & 41.076652 & 40.178240 & 32.933519 \\ \mathrm{C} & 42.538803 & 40.602651 & 34.934101 \\ \mathrm{H} & 41.609238 & 41.133996 & 35.019201 \\ \mathrm{C} & 43.465464 & 40.629174 & 35.958356 \\ \mathrm{H} & 43.237787 & 41.199470 & 36.842005 \\ \mathrm{C} & 41.404665 & 35.997847 & 34.466925 \\ \mathrm{H} & 41.061063 & 35.640375 & 35.431809 \\ \mathrm{H} & 42.421267 & 36.354701 & 34.596803 \\ \mathrm{C} & 40.529944 & 37.169595 & 34.010919 \\ \mathrm{H} & 40.676956 & 38.000421 & 34.694171 \\ \mathrm{H} & 40.833784 & 37.559685 & 33.044412 \\ \mathrm{C} & 39.039805 & 36.919762 & 33.945992 \\ \mathrm{O} & 38.535577 & 35.848423 & 34.075870 \\ \mathrm{O} & 38.298453 & 37.978945 & 33.726198 \\ \mathrm{H} & 38.776215 & 38.815434 & 33.608811 \\ \mathrm{~N} & 32.651360 & 46.711891 & 29.422566 \\ \mathrm{H} & 33.503214 & 46.204545 & 29.375978 \\ \mathrm{C} & 31.456557 & 45.989610 & 29.687492 \\ \mathrm{C} & 31.455777 & 44.593963 & 29.056467 \\ \mathrm{~S} & 32.308551 & 43.212128 & 29.920515\end{array}$




\begin{tabular}{|c|c|c|c|}
\hline C & 34.043057 & 43.329907 & 29.377905 \\
\hline C & 34.597713 & 42.129897 & 28.784444 \\
\hline C & 35.968563 & 41.627393 & 29.027614 \\
\hline C & 36.698080 & 41.597604 & 30.234688 \\
\hline C & 36.259563 & 41.425111 & 31.580818 \\
\hline C & 37.168496 & 41.080945 & 32.557223 \\
\hline C & 38.569723 & 40.903188 & 32.297655 \\
\hline 0 & 39.366542 & 40.443930 & 33.190352 \\
\hline C & 39.001940 & 41.190782 & 30.968547 \\
\hline C & 38.094958 & 41.484069 & 29.982544 \\
\hline C & 38.354032 & 41.619827 & 28.506031 \\
\hline C & 36.972394 & 41.941333 & 27.915296 \\
\hline $\mathrm{H}$ & 36.901935 & 42.999807 & 27.667988 \\
\hline $\mathrm{H}$ & 36.767198 & 41.384657 & 27.004872 \\
\hline $\mathrm{H}$ & 38.727722 & 40.686947 & 28.097797 \\
\hline $\mathrm{H}$ & 39.088528 & 42.388792 & 28.289201 \\
\hline $\mathrm{H}$ & 40.049345 & 41.086376 & 30.740408 \\
\hline $\mathrm{H}$ & 36.829436 & 40.900465 & 33.561218 \\
\hline $\mathrm{H}$ & 35.213309 & 41.483651 & 31.822441 \\
\hline $\mathrm{H}$ & 33.851091 & 41.353891 & 28.722858 \\
\hline 0 & 34.643960 & 44.369112 & 29.512077 \\
\hline $\mathrm{H}$ & 30.439140 & 44.226655 & 29.031546 \\
\hline $\mathrm{H}$ & 31.829366 & 44.657691 & 28.042973 \\
\hline $\mathrm{H}$ & 30.609725 & 46.507534 & 29.240297 \\
\hline C & 31.076069 & 45.930021 & 31.167115 \\
\hline 0 & 29.962428 & 45.705263 & 31.512299 \\
\hline $\mathrm{H}$ & 32.703871 & 47.674462 & 29.625082 \\
\hline $\mathrm{H}$ & 31.843808 & 46.133911 & 31.861288 \\
\hline $\mathrm{H}$ & 45.444335 & 40.074683 & 36.620304 \\
\hline $\mathrm{H}$ & 41.418925 & 35.137672 & 33.761087 \\
\hline \multicolumn{4}{|c|}{ C7-C10-C11-C12 50 } \\
\hline C & 44.682309 & 39.962073 & 35.847908 \\
\hline C & 44.953637 & 39.270813 & 34.673837 \\
\hline $\mathrm{H}$ & 45.882433 & 38.743914 & 34.544144 \\
\hline C & 44.030075 & 39.244523 & 33.649790 \\
\hline $\mathrm{H}$ & 44.248213 & 38.727424 & 32.734399 \\
\hline C & 42.820575 & 39.923665 & 33.769525 \\
\hline 0 & 41.983572 & 39.909308 & 32.737021 \\
\hline $\mathrm{H}$ & 41.070690 & 40.179911 & 32.955140 \\
\hline C & 42.541009 & 40.602579 & 34.950018 \\
\hline $\mathrm{H}$ & 41.605186 & 41.120765 & 35.043857 \\
\hline C & 43.469985 & 40.624553 & 35.971845 \\
\hline $\mathrm{H}$ & 43.238440 & 41.179385 & 36.864312 \\
\hline
\end{tabular}




\begin{tabular}{|c|c|c|c|}
\hline$C$ & 41.425372 & 36.009472 & 34.479521 \\
\hline $\mathrm{H}$ & 41.083890 & 35.652741 & 35.445666 \\
\hline $\mathrm{H}$ & 42.444395 & 36.361530 & 34.604847 \\
\hline C & 40.555416 & 37.186225 & 34.027126 \\
\hline $\mathrm{H}$ & 40.711655 & 38.016543 & 34.708630 \\
\hline $\mathrm{H}$ & 40.857827 & 37.573929 & 33.059346 \\
\hline C & 39.062549 & 36.946718 & 33.966295 \\
\hline 0 & 38.555285 & 35.875291 & 34.097128 \\
\hline 0 & 38.329538 & 38.009565 & 33.753913 \\
\hline $\mathrm{H}$ & 38.812156 & 38.850954 & 33.634446 \\
\hline $\mathrm{N}$ & 32.619041 & 46.737221 & 29.444547 \\
\hline $\mathrm{H}$ & 33.474028 & 46.241032 & 29.358632 \\
\hline C & 31.436210 & 45.997315 & 29.709700 \\
\hline C & 31.462921 & 44.600848 & 29.083578 \\
\hline$S$ & 32.365319 & 43.251712 & 29.951802 \\
\hline$C$ & 34.072586 & 43.369589 & 29.340137 \\
\hline C & 34.636967 & 42.119681 & 28.847556 \\
\hline$C$ & 36.021408 & 41.673266 & 29.070820 \\
\hline C & 36.738353 & 41.611268 & 30.288636 \\
\hline C & 36.289054 & 41.376654 & 31.618542 \\
\hline C & 37.196294 & 41.019768 & 32.593411 \\
\hline C & 38.602320 & 40.895087 & 32.347426 \\
\hline 0 & 39.402489 & 40.417247 & 33.241160 \\
\hline C & 39.041376 & 41.234878 & 31.037618 \\
\hline C & 38.136275 & 41.545413 & 30.049054 \\
\hline C & 38.407916 & 41.719832 & 28.577812 \\
\hline C & 37.027319 & 42.033821 & 27.977649 \\
\hline $\mathrm{H}$ & 36.943451 & 43.096343 & 27.752643 \\
\hline $\mathrm{H}$ & 36.838808 & 41.493064 & 27.053814 \\
\hline $\mathrm{H}$ & 38.798940 & 40.799318 & 28.156605 \\
\hline $\mathrm{H}$ & 39.133684 & 42.502555 & 28.380561 \\
\hline $\mathrm{H}$ & 40.092209 & 41.158588 & 30.814951 \\
\hline $\mathrm{H}$ & 36.847955 & 40.796129 & 33.585613 \\
\hline $\mathrm{H}$ & 35.238337 & 41.392798 & 31.845969 \\
\hline $\mathrm{H}$ & 33.900718 & 41.332943 & 28.846618 \\
\hline 0 & 34.649863 & 44.427475 & 29.336968 \\
\hline $\mathrm{H}$ & 30.457056 & 44.204239 & 29.073385 \\
\hline $\mathrm{H}$ & 31.819355 & 44.673408 & 28.064227 \\
\hline $\mathrm{H}$ & 30.577947 & 46.498278 & 29.264922 \\
\hline C & 31.061471 & 45.931996 & 31.189753 \\
\hline 0 & 29.952219 & 45.692279 & 31.536860 \\
\hline $\mathrm{H}$ & 32.664932 & 47.697037 & 29.662576 \\
\hline $\mathrm{H}$ & 31.829138 & 46.144523 & 31.882022 \\
\hline $\mathrm{H}$ & 45.455911 & 40.079779 & 36.620907 \\
\hline
\end{tabular}




$\begin{array}{llll}H & 41.432480 & 35.150421 & 33.772239\end{array}$

\begin{tabular}{|c|c|c|c|}
\hline \multicolumn{4}{|c|}{$\mathrm{C} 7-\mathrm{C} 10-\mathrm{C} 11-\mathrm{C} 1260^{\circ}$} \\
\hline C & 44.679325 & 39.952752 & 35.853299 \\
\hline C & 44.952877 & 39.258561 & 34.681718 \\
\hline $\mathrm{H}$ & 45.886953 & 38.738979 & 34.556698 \\
\hline C & 44.030827 & 39.227543 & 33.656590 \\
\hline $\mathrm{H}$ & 44.252227 & 38.712464 & 32.740541 \\
\hline C & 42.818363 & 39.902563 & 33.774416 \\
\hline 0 & 41.981998 & 39.883053 & 32.742394 \\
\hline $\mathrm{H}$ & 41.065961 & 40.150361 & 32.960758 \\
\hline C & 42.533712 & 40.578163 & 34.955863 \\
\hline $\mathrm{H}$ & 41.594956 & 41.091379 & 35.048676 \\
\hline C & 43.462857 & 40.606972 & 35.977355 \\
\hline $\mathrm{H}$ & 43.229958 & 41.162353 & 36.869291 \\
\hline C & 41.402794 & 35.990967 & 34.475887 \\
\hline $\mathrm{H}$ & 41.050095 & 35.633015 & 35.437544 \\
\hline $\mathrm{H}$ & 42.421815 & 36.338954 & 34.614167 \\
\hline C & 40.540671 & 37.171810 & 34.020730 \\
\hline $\mathrm{H}$ & 40.686717 & 37.995529 & 34.712673 \\
\hline $\mathrm{H}$ & 40.857564 & 37.567884 & 33.061131 \\
\hline C & 39.048267 & 36.934601 & 33.938111 \\
\hline 0 & 38.538244 & 35.861808 & 34.051594 \\
\hline 0 & 38.321377 & 37.999765 & 33.725263 \\
\hline $\mathrm{H}$ & 38.807686 & 38.844006 & 33.612897 \\
\hline $\mathrm{N}$ & 32.662054 & 46.786074 & 29.379187 \\
\hline $\mathrm{H}$ & 33.512780 & 46.297967 & 29.232914 \\
\hline C & 31.491728 & 46.035868 & 29.646068 \\
\hline C & 31.536014 & 44.642107 & 29.021550 \\
\hline$S$ & 32.455203 & 43.311835 & 29.892357 \\
\hline C & 34.135910 & 43.423354 & 29.220100 \\
\hline C & 34.728718 & 42.147663 & 28.820560 \\
\hline C & 36.131209 & 41.773756 & 29.034468 \\
\hline C & 36.813050 & 41.673228 & 30.270008 \\
\hline C & 36.331651 & 41.395598 & 31.579340 \\
\hline C & 37.216780 & 41.000105 & 32.561313 \\
\hline C & 38.626688 & 40.888477 & 32.346156 \\
\hline 0 & 39.410376 & 40.383601 & 33.246954 \\
\hline C & 39.095846 & 41.259050 & 31.057110 \\
\hline C & 38.214780 & 41.615919 & 30.059727 \\
\hline C & 38.519685 & 41.811454 & 28.596278 \\
\hline C & 37.152629 & 42.123791 & 27.959964 \\
\hline $\mathrm{H}$ & 37.077557 & 43.182582 & 27.718415 \\
\hline $\mathrm{H}$ & 36.984563 & 41.572731 & 27.037973 \\
\hline
\end{tabular}




\begin{tabular}{|c|c|c|c|}
\hline $\mathrm{H}$ & 38.926750 & 40.895383 & 28.180605 \\
\hline $\mathrm{H}$ & 39.244974 & 42.600345 & 28.422296 \\
\hline $\mathrm{H}$ & 40.150773 & 41.181884 & 30.855460 \\
\hline $\mathrm{H}$ & 36.845741 & 40.745452 & 33.537895 \\
\hline $\mathrm{H}$ & 35.276172 & 41.398167 & 31.785700 \\
\hline $\mathrm{H}$ & 34.014990 & 41.341698 & 28.814687 \\
\hline 0 & 34.681553 & 44.488323 & 29.082932 \\
\hline H & 30.531900 & 44.244567 & 29.001589 \\
\hline $\mathrm{H}$ & 31.910743 & 44.706445 & 28.010124 \\
\hline $\mathrm{H}$ & 30.623607 & 46.524791 & 29.207292 \\
\hline C & 31.124287 & 45.961045 & 31.125069 \\
\hline 0 & 30.015090 & 45.715965 & 31.469382 \\
\hline $\mathrm{H}$ & 32.709408 & 47.743988 & 29.603999 \\
\hline $\mathrm{H}$ & 31.893810 & 46.171919 & 31.816020 \\
\hline $\mathrm{H}$ & 45.453552 & 40.077812 & 36.624070 \\
\hline $\mathrm{H}$ & 41.414598 & 35.133163 & 33.766855 \\
\hline \multicolumn{4}{|c|}{$\mathrm{C} 7-\mathrm{C} 10-\mathrm{C} 11-\mathrm{C} 1270^{\circ}$} \\
\hline C & 44.694139 & 39.935142 & 35.860909 \\
\hline C & 44.969998 & 39.243394 & 34.688129 \\
\hline $\mathrm{H}$ & 45.899524 & 38.717679 & 34.559680 \\
\hline C & 44.048264 & 39.214092 & 33.662996 \\
\hline $\mathrm{H}$ & 44.270647 & 38.696740 & 32.748926 \\
\hline C & 42.836177 & 39.889538 & 33.779992 \\
\hline 0 & 41.998921 & 39.869561 & 32.749453 \\
\hline $\mathrm{H}$ & 41.081802 & 40.133547 & 32.971155 \\
\hline C & 42.554037 & 40.570495 & 34.959358 \\
\hline $\mathrm{H}$ & 41.616818 & 41.086486 & 35.051070 \\
\hline C & 43.481096 & 40.597275 & 35.982021 \\
\hline $\mathrm{H}$ & 43.247868 & 41.155608 & 36.872021 \\
\hline C & 41.420332 & 35.994457 & 34.481761 \\
\hline $\mathrm{H}$ & 41.074613 & 35.631692 & 35.444081 \\
\hline $\mathrm{H}$ & 42.439445 & 36.343516 & 34.613782 \\
\hline C & 40.552699 & 37.173901 & 34.035028 \\
\hline $\mathrm{H}$ & 40.701417 & 37.997527 & 34.726188 \\
\hline $\mathrm{H}$ & 40.862103 & 37.572852 & 33.074337 \\
\hline C & 39.060340 & 36.934607 & 33.960147 \\
\hline 0 & 38.552362 & 35.860812 & 34.078318 \\
\hline 0 & 38.331769 & 37.998558 & 33.748886 \\
\hline $\mathrm{H}$ & 38.820466 & 38.842540 & 33.634792 \\
\hline$N$ & 32.646554 & 46.799553 & 29.367434 \\
\hline $\mathrm{H}$ & 33.493222 & 46.308449 & 29.208693 \\
\hline C & 31.476085 & 46.050511 & 29.638298 \\
\hline C & 31.527785 & 44.656533 & 29.012747 \\
\hline
\end{tabular}




\begin{tabular}{llll} 
S & 32.434463 & 43.328427 & 29.899847 \\
C & 34.118184 & 43.431742 & 29.240748 \\
C & 34.737920 & 42.151056 & 28.907737 \\
C & 36.161249 & 41.860245 & 29.085737 \\
C & 36.839622 & 41.700096 & 30.312441 \\
C & 36.356155 & 41.387942 & 31.612439 \\
C & 37.242203 & 40.979749 & 32.588061 \\
C & 38.651528 & 40.880140 & 32.372377 \\
O & 39.437435 & 40.364966 & 33.267894 \\
C & 39.121412 & 41.263385 & 31.086516 \\
C & 38.241057 & 41.646143 & 30.098247 \\
C & 38.542411 & 41.871923 & 28.637120 \\
C & 37.172397 & 42.182706 & 27.999413 \\
H & 37.103730 & 43.236315 & 27.735641 \\
H & 36.996844 & 41.612039 & 27.090176 \\
H & 38.962366 & 40.968938 & 28.206084 \\
H & 39.258519 & 42.672351 & 28.477563 \\
H & 40.175887 & 41.186268 & 30.882722 \\
H & 36.870216 & 40.704696 & 33.558618 \\
H & 35.300308 & 41.380874 & 31.814838 \\
H & 34.051129 & 41.324943 & 28.859242 \\
O & 34.650232 & 44.495225 & 29.047610 \\
H & 30.524212 & 44.259811 & 28.969335 \\
H & 31.915735 & 44.734254 & 28.005881 \\
H & 30.602659 & 46.531865 & 29.202370 \\
C & 31.108095 & 45.980122 & 31.117299 \\
O & 29.998111 & 45.741745 & 31.460619 \\
H & 32.702167 & 47.753546 & 29.606724 \\
H & 31.878130 & 46.183844 & 31.809829 \\
H & 45.466784 & 40.057264 & 36.633895 \\
H & 41.429128 & 35.139618 & 33.769324 \\
& & & \\
\hline
\end{tabular}

$\begin{array}{lrrr}\text { C7-C10-C11-C12 80 } & & \\ \text { C } & 44.721625 & 39.927565 & 35.856680 \\ \text { C } & 45.007339 & 39.237820 & 34.685677 \\ \text { H } & 45.941985 & 38.720902 & 34.560135 \\ \text { C } & 44.090308 & 39.203718 & 33.656598 \\ \text { H } & 44.318516 & 38.684886 & 32.744932 \\ \text { C } & 42.871313 & 39.865843 & 33.772687 \\ \text { O } & 42.036040 & 39.842110 & 32.740895 \\ \text { H } & 41.117072 & 40.097541 & 32.968631 \\ \text { C } & 42.577942 & 40.541767 & 34.952821 \\ \text { H } & 41.633102 & 41.043369 & 35.043972 \\ \text { C } & 43.500407 & 40.574524 & 35.978729\end{array}$




\begin{tabular}{|c|c|c|c|}
\hline $\mathrm{H}$ & 43.257362 & 41.123005 & 36.872482 \\
\hline C & 41.443000 & 35.956413 & 34.473461 \\
\hline $\mathrm{H}$ & 41.105077 & 35.592728 & 35.438123 \\
\hline $\mathrm{H}$ & 42.463525 & 36.303351 & 34.601004 \\
\hline C & 40.576364 & 37.136463 & 34.029813 \\
\hline $\mathrm{H}$ & 40.726617 & 37.958282 & 34.723203 \\
\hline $\mathrm{H}$ & 40.886487 & 37.537156 & 33.070164 \\
\hline C & 39.083738 & 36.898658 & 33.948325 \\
\hline 0 & 38.572683 & 35.824695 & 34.049786 \\
\hline 0 & 38.357761 & 37.966264 & 33.748263 \\
\hline $\mathrm{H}$ & 38.853111 & 38.807425 & 33.640876 \\
\hline N & 32.628406 & 46.763770 & 29.332570 \\
\hline $\mathrm{H}$ & 33.475091 & 46.272063 & 29.177621 \\
\hline C & 31.454467 & 46.020294 & 29.606116 \\
\hline C & 31.504364 & 44.622110 & 28.990154 \\
\hline$S$ & 32.413177 & 43.301817 & 29.885746 \\
\hline C & 34.105147 & 43.416560 & 29.259115 \\
\hline C & 34.761829 & 42.137948 & 29.013349 \\
\hline C & 36.198324 & 41.928263 & 29.165212 \\
\hline C & 36.881858 & 41.712479 & 30.374846 \\
\hline C & 36.403744 & 41.381013 & 31.671973 \\
\hline C & 37.295838 & 40.958779 & 32.635098 \\
\hline C & 38.701442 & 40.851466 & 32.406332 \\
\hline 0 & 39.489203 & 40.318091 & 33.290190 \\
\hline C & 39.165323 & 41.240642 & 31.119560 \\
\hline C & 38.281789 & 41.653455 & 30.147244 \\
\hline C & 38.572858 & 41.912350 & 28.688646 \\
\hline C & 37.193051 & 42.197929 & 28.053567 \\
\hline $\mathrm{H}$ & 37.122116 & 43.235965 & 27.736286 \\
\hline $\mathrm{H}$ & 37.004456 & 41.581525 & 27.176504 \\
\hline $\mathrm{H}$ & 39.023460 & 41.031116 & 28.244166 \\
\hline $\mathrm{H}$ & 39.262554 & 42.738411 & 28.542485 \\
\hline $\mathrm{H}$ & 40.217336 & 41.156173 & 30.906764 \\
\hline $\mathrm{H}$ & 36.930045 & 40.659579 & 33.600586 \\
\hline $\mathrm{H}$ & 35.349949 & 41.380789 & 31.883880 \\
\hline $\mathrm{H}$ & 34.107736 & 41.291343 & 28.914098 \\
\hline 0 & 34.623472 & 44.479766 & 29.027677 \\
\hline $\mathrm{H}$ & 30.501978 & 44.223040 & 28.947933 \\
\hline $\mathrm{H}$ & 31.891141 & 44.693028 & 27.982227 \\
\hline $\mathrm{H}$ & 30.585168 & 46.506601 & 29.166925 \\
\hline C & 31.086652 & 45.961151 & 31.085954 \\
\hline 0 & 29.978393 & 45.720663 & 31.434259 \\
\hline $\mathrm{H}$ & 32.679906 & 47.721452 & 29.561183 \\
\hline $\mathrm{H}$ & 31.857385 & 46.173732 & 31.774442 \\
\hline
\end{tabular}




$\begin{array}{llll}\mathrm{H} & 45.487788 & 40.049755 & 36.635865 \\ \mathrm{H} & 41.447126 & 35.102839 & 33.760759\end{array}$

\begin{tabular}{|c|c|c|c|}
\hline \multicolumn{4}{|c|}{$\mathrm{C} 7-\mathrm{C} 10-\mathrm{C} 11-\mathrm{C} 1290^{\circ}$} \\
\hline C & 44.706279 & 39.921324 & 35.833527 \\
\hline C & 44.993502 & 39.216316 & 34.671764 \\
\hline $\mathrm{H}$ & 45.929355 & 38.700506 & 34.552100 \\
\hline C & 44.078926 & 39.169500 & 33.640312 \\
\hline $\mathrm{H}$ & 44.307528 & 38.637005 & 32.736567 \\
\hline C & 42.860076 & 39.832310 & 33.747971 \\
\hline 0 & 42.024349 & 39.797874 & 32.716497 \\
\hline $\mathrm{H}$ & 41.102751 & 40.036772 & 32.950935 \\
\hline C & 42.562349 & 40.519647 & 34.920371 \\
\hline $\mathrm{H}$ & 41.617847 & 41.023094 & 35.003158 \\
\hline C & 43.483866 & 40.567690 & 35.946105 \\
\hline $\mathrm{H}$ & 43.239155 & 41.128816 & 36.831180 \\
\hline C & 41.453429 & 35.920015 & 34.505917 \\
\hline $\mathrm{H}$ & 41.121716 & 35.555748 & 35.471898 \\
\hline $\mathrm{H}$ & 42.472928 & 36.272618 & 34.624804 \\
\hline C & 40.578402 & 37.094587 & 34.064479 \\
\hline $\mathrm{H}$ & 40.737643 & 37.924193 & 34.746359 \\
\hline $\mathrm{H}$ & 40.874989 & 37.487292 & 33.097008 \\
\hline C & 39.086010 & 36.848655 & 34.012007 \\
\hline 0 & 38.586492 & 35.772647 & 34.144356 \\
\hline 0 & 38.349093 & 37.907061 & 33.806020 \\
\hline $\mathrm{H}$ & 38.839966 & 38.748365 & 33.673486 \\
\hline$N$ & 32.544567 & 46.849263 & 29.416424 \\
\hline $\mathrm{H}$ & 33.385490 & 46.364994 & 29.214498 \\
\hline C & 31.372497 & 46.093515 & 29.677330 \\
\hline C & 31.434329 & 44.702956 & 29.037136 \\
\hline S & 32.328720 & 43.363469 & 29.918150 \\
\hline C & 34.024042 & 43.486771 & 29.308680 \\
\hline C & 34.702298 & 42.206835 & 29.155590 \\
\hline C & 36.143909 & 42.030712 & 29.276607 \\
\hline C & 36.844567 & 41.735947 & 30.455917 \\
\hline C & 36.389610 & 41.419119 & 31.765091 \\
\hline C & 37.289814 & 40.949831 & 32.698929 \\
\hline C & 38.682666 & 40.790023 & 32.432106 \\
\hline 0 & 39.478870 & 40.239986 & 33.297459 \\
\hline C & 39.126706 & 41.163452 & 31.132658 \\
\hline C & 38.234556 & 41.614485 & 30.188000 \\
\hline C & 38.499337 & 41.890938 & 28.727982 \\
\hline C & 37.107095 & 42.176200 & 28.116458 \\
\hline $\mathrm{H}$ & 37.061011 & 43.182896 & 27.708867 \\
\hline
\end{tabular}




$\begin{array}{llll}\text { H } & 36.867313 & 41.493093 & 27.302827 \\ \mathrm{H} & 38.959847 & 41.026042 & 28.262700 \\ \mathrm{H} & 39.173389 & 42.731257 & 28.587859 \\ \mathrm{H} & 40.167721 & 41.033846 & 30.890078 \\ \mathrm{H} & 36.937197 & 40.654769 & 33.670678 \\ \mathrm{H} & 35.343426 & 41.463731 & 32.008110 \\ \mathrm{H} & 34.062055 & 41.354918 & 29.016325 \\ \mathrm{O} & 34.521750 & 44.547613 & 29.026959 \\ \mathrm{H} & 30.433016 & 44.302227 & 28.961005 \\ \mathrm{H} & 31.859054 & 44.792633 & 28.046578 \\ \mathrm{H} & 30.505645 & 46.581103 & 29.236863 \\ \mathrm{C} & 31.013269 & 46.007963 & 31.158306 \\ \mathrm{O} & 29.907903 & 45.760088 & 31.516929 \\ \mathrm{H} & 32.599696 & 47.803031 & 29.649375 \\ \mathrm{H} & 31.789492 & 46.213273 & 31.840219 \\ \mathrm{H} & 45.469584 & 40.052478 & 36.613851 \\ \mathrm{H} & 41.456371 & 35.066288 & 33.792593\end{array}$

$\begin{array}{lrll}\text { C7-C10-C11-C12 } 100^{\circ} & & \\ \mathrm{C} & 44.738809 & 39.901204 & 35.865281 \\ \mathrm{C} & 45.028317 & 39.189700 & 34.708954 \\ \mathrm{H} & 45.962566 & 38.670413 & 34.595862 \\ \mathrm{C} & 44.114365 & 39.136084 & 33.677727 \\ \mathrm{H} & 44.342817 & 38.598731 & 32.776943 \\ \mathrm{C} & 42.895793 & 39.799292 & 33.780051 \\ \mathrm{O} & 42.060012 & 39.754434 & 32.749598 \\ \mathrm{H} & 41.139865 & 40.005001 & 32.985379 \\ \mathrm{C} & 42.596794 & 40.496279 & 34.946696 \\ \mathrm{H} & 41.652366 & 41.000079 & 35.026328 \\ \mathrm{C} & 43.517563 & 40.551050 & 35.971895 \\ \mathrm{H} & 43.272285 & 41.118651 & 36.852575 \\ \mathrm{C} & 41.469479 & 35.903673 & 34.490081 \\ \mathrm{H} & 41.136643 & 35.535505 & 35.454039 \\ \mathrm{H} & 42.487211 & 36.261039 & 34.607709 \\ \mathrm{C} & 40.594365 & 37.077806 & 34.050705 \\ \mathrm{H} & 40.746874 & 37.902898 & 34.739261 \\ \mathrm{H} & 40.901976 & 37.477453 & 33.089602 \\ \mathrm{C} & 39.102219 & 36.833578 & 33.981347 \\ \mathrm{O} & 38.596016 & 35.757522 & 34.082542 \\ \mathrm{O} & 38.371638 & 37.900504 & 33.793438 \\ \mathrm{H} & 38.872953 & 38.738671 & 33.693784 \\ \mathrm{~N} & 32.586088 & 46.791974 & 29.320306 \\ \mathrm{H} & 33.429434 & 46.307252 & 29.129108 \\ \mathrm{C} & 31.407727 & 46.043236 & 29.576248\end{array}$




\begin{tabular}{|c|c|c|c|}
\hline C & 31.462868 & 44.655100 & 28.938774 \\
\hline$S$ & 32.370214 & 43.330754 & 29.820785 \\
\hline C & 34.079015 & 43.452350 & 29.255615 \\
\hline C & 34.780328 & 42.177564 & 29.216707 \\
\hline C & 36.228263 & 42.065806 & 29.330850 \\
\hline C & 36.923302 & 41.719694 & 30.497892 \\
\hline C & 36.461133 & 41.396296 & 31.801603 \\
\hline C & 37.356442 & 40.923272 & 32.737244 \\
\hline C & 38.751429 & 40.775595 & 32.477328 \\
\hline 0 & 39.543945 & 40.227051 & 33.345982 \\
\hline C & 39.201802 & 41.145941 & 31.178044 \\
\hline C & 38.312421 & 41.593430 & 30.230530 \\
\hline C & 38.577860 & 41.894587 & 28.775626 \\
\hline C & 37.176017 & 42.088223 & 28.149053 \\
\hline $\mathrm{H}$ & 37.111177 & 43.030111 & 27.612872 \\
\hline $\mathrm{H}$ & 36.938426 & 41.298951 & 27.435622 \\
\hline $\mathrm{H}$ & 39.111112 & 41.073924 & 28.310134 \\
\hline $\mathrm{H}$ & 39.185776 & 42.787386 & 28.654540 \\
\hline $\mathrm{H}$ & 40.244378 & 41.018841 & 30.941085 \\
\hline $\mathrm{H}$ & 37.000618 & 40.624817 & 33.706822 \\
\hline $\mathrm{H}$ & 35.413808 & 41.444388 & 32.037579 \\
\hline $\mathrm{H}$ & 34.172736 & 41.308548 & 29.041038 \\
\hline 0 & 34.576990 & 44.499961 & 28.928555 \\
\hline $\mathrm{H}$ & 30.462445 & 44.246466 & 28.885861 \\
\hline $\mathrm{H}$ & 31.869408 & 44.749804 & 27.940432 \\
\hline $\mathrm{H}$ & 30.550551 & 46.536162 & 29.124041 \\
\hline C & 31.027376 & 45.971635 & 31.058521 \\
\hline 0 & 29.914287 & 45.729687 & 31.398976 \\
\hline $\mathrm{H}$ & 32.636433 & 47.744664 & 29.559874 \\
\hline $\mathrm{H}$ & 31.788841 & 46.188565 & 31.752970 \\
\hline $\mathrm{H}$ & 45.498164 & 40.031921 & 36.648418 \\
\hline $\mathrm{H}$ & 41.475475 & 35.053381 & 33.772528 \\
\hline \multicolumn{4}{|c|}{ C7-C10-C11-C12 110 } \\
\hline C & 44.742559 & 39.891655 & 35.858442 \\
\hline C & 45.032764 & 39.184640 & 34.698701 \\
\hline $\mathrm{H}$ & 45.969018 & 38.671789 & 34.578647 \\
\hline C & 44.117832 & 39.129258 & 33.668624 \\
\hline $\mathrm{H}$ & 44.350199 & 38.598849 & 32.764768 \\
\hline C & 42.899013 & 39.791664 & 33.772178 \\
\hline 0 & 42.064734 & 39.752070 & 32.739619 \\
\hline $\mathrm{H}$ & 41.143630 & 39.988166 & 32.978058 \\
\hline C & 42.597538 & 40.480714 & 34.942632 \\
\hline $\mathrm{H}$ & 41.651825 & 40.982292 & 35.022427 \\
\hline
\end{tabular}




\begin{tabular}{|c|c|c|c|}
\hline C & 43.518635 & 40.535739 & 35.968184 \\
\hline $\mathrm{H}$ & 43.270302 & 41.095834 & 36.852719 \\
\hline C & 41.515464 & 35.924580 & 34.541758 \\
\hline $\mathrm{H}$ & 41.211816 & 35.551597 & 35.513226 \\
\hline $\mathrm{H}$ & 42.533414 & 36.287637 & 34.636025 \\
\hline C & 40.619023 & 37.091488 & 34.129388 \\
\hline $\mathrm{H}$ & 40.786123 & 37.919707 & 34.811150 \\
\hline $\mathrm{H}$ & 40.888726 & 37.491677 & 33.157692 \\
\hline C & 39.129219 & 36.831537 & 34.111286 \\
\hline 0 & 38.640558 & 35.754179 & 34.268056 \\
\hline 0 & 38.381678 & 37.882941 & 33.907037 \\
\hline $\mathrm{H}$ & 38.873814 & 38.720909 & 33.764177 \\
\hline $\mathrm{N}$ & 32.474872 & 46.901448 & 29.433153 \\
\hline $\mathrm{H}$ & 33.314628 & 46.411439 & 29.237803 \\
\hline C & 31.300472 & 46.152256 & 29.704728 \\
\hline C & 31.344364 & 44.768802 & 29.053241 \\
\hline S & 32.215526 & 43.420611 & 29.931463 \\
\hline C & 33.934234 & 43.541182 & 29.400326 \\
\hline C & 34.652807 & 42.275167 & 29.449418 \\
\hline C & 36.106777 & 42.193657 & 29.519858 \\
\hline C & 36.836680 & 41.769162 & 30.639773 \\
\hline C & 36.419487 & 41.436679 & 31.956429 \\
\hline C & 37.339814 & 40.927487 & 32.847023 \\
\hline C & 38.718651 & 40.748961 & 32.526575 \\
\hline 0 & 39.536341 & 40.191595 & 33.363749 \\
\hline C & 39.122033 & 41.111915 & 31.210215 \\
\hline C & 38.206577 & 41.596676 & 30.307177 \\
\hline C & 38.416882 & 41.926447 & 28.849066 \\
\hline C & 36.992478 & 42.154173 & 28.289787 \\
\hline $\mathrm{H}$ & 36.928819 & 43.082382 & 27.730977 \\
\hline $\mathrm{H}$ & 36.693586 & 41.353577 & 27.612355 \\
\hline $\mathrm{H}$ & 38.922548 & 41.116831 & 28.335049 \\
\hline $\mathrm{H}$ & 39.028121 & 42.816688 & 28.727301 \\
\hline $\mathrm{H}$ & 40.148035 & 40.950388 & 30.926492 \\
\hline $\mathrm{H}$ & 37.020137 & 40.625680 & 33.828135 \\
\hline $\mathrm{H}$ & 35.387165 & 41.516024 & 32.243775 \\
\hline $\mathrm{H}$ & 34.071943 & 41.405902 & 29.196081 \\
\hline 0 & 34.428025 & 44.578758 & 29.036709 \\
\hline $\mathrm{H}$ & 30.339434 & 44.376949 & 28.981884 \\
\hline $\mathrm{H}$ & 31.763891 & 44.867023 & 28.061022 \\
\hline $\mathrm{H}$ & 30.432968 & 46.652255 & 29.281914 \\
\hline C & 30.948752 & 46.069895 & 31.191020 \\
\hline 0 & 29.838791 & 45.856021 & 31.555115 \\
\hline $\mathrm{H}$ & 32.539019 & 47.849755 & 29.684843 \\
\hline
\end{tabular}




$\begin{array}{llll}\mathrm{H} & 31.730751 & 46.266580 & 31.869534 \\ \mathrm{H} & 45.504835 & 40.027746 & 36.638346 \\ \mathrm{H} & 41.507893 & 35.076403 & 33.822115\end{array}$

\begin{tabular}{|c|c|c|c|}
\hline \multicolumn{4}{|c|}{ C7-C10-C11-C12 120 } \\
\hline C & 44.766386 & 39.847525 & 35.864902 \\
\hline C & 45.065154 & 39.125580 & 34.717418 \\
\hline $\mathrm{H}$ & 46.000886 & 38.608052 & 34.614061 \\
\hline C & 44.160838 & 39.059830 & 33.678235 \\
\hline $\mathrm{H}$ & 44.399666 & 38.520853 & 32.780726 \\
\hline C & 42.943664 & 39.726874 & 33.761074 \\
\hline 0 & 42.118792 & 39.679472 & 32.720684 \\
\hline $\mathrm{H}$ & 41.197550 & 39.917953 & 32.954776 \\
\hline C & 42.632450 & 40.430474 & 34.920031 \\
\hline $\mathrm{H}$ & 41.686897 & 40.934491 & 34.982121 \\
\hline C & 43.544141 & 40.497429 & 35.952877 \\
\hline $\mathrm{H}$ & 43.289764 & 41.066993 & 36.829653 \\
\hline C & 41.522674 & 35.878970 & 34.524852 \\
\hline $\mathrm{H}$ & 41.215994 & 35.506837 & 35.496279 \\
\hline $\mathrm{H}$ & 42.542404 & 36.237547 & 34.626428 \\
\hline C & 40.630014 & 37.046828 & 34.108176 \\
\hline $\mathrm{H}$ & 40.789280 & 37.874383 & 34.791986 \\
\hline $\mathrm{H}$ & 40.910351 & 37.446270 & 33.139091 \\
\hline C & 39.139605 & 36.787440 & 34.067665 \\
\hline 0 & 38.644037 & 35.710405 & 34.201749 \\
\hline 0 & 38.400011 & 37.844792 & 33.865253 \\
\hline $\mathrm{H}$ & 38.901979 & 38.678963 & 33.734326 \\
\hline $\mathrm{N}$ & 32.548824 & 46.897922 & 29.288870 \\
\hline $\mathrm{H}$ & 33.380097 & 46.396742 & 29.087057 \\
\hline C & 31.359138 & 46.167573 & 29.548170 \\
\hline C & 31.401092 & 44.773008 & 28.915863 \\
\hline$S$ & 32.281909 & 43.443660 & 29.816365 \\
\hline C & 34.009403 & 43.571864 & 29.314411 \\
\hline C & 34.748909 & 42.330074 & 29.500809 \\
\hline C & 36.206780 & 42.285014 & 29.563646 \\
\hline C & 36.935414 & 41.811786 & 30.665529 \\
\hline C & 36.516666 & 41.480769 & 31.981951 \\
\hline C & 37.425670 & 40.929864 & 32.856677 \\
\hline C & 38.796874 & 40.715355 & 32.523076 \\
\hline 0 & 39.599540 & 40.126750 & 33.351409 \\
\hline C & 39.200068 & 41.067058 & 31.204359 \\
\hline C & 38.292997 & 41.592414 & 30.314978 \\
\hline C & 38.492664 & 41.925686 & 28.856885 \\
\hline C & 37.060923 & 42.149574 & 28.316504 \\
\hline
\end{tabular}




$\begin{array}{llll}\text { H } & 37.001350 & 43.034549 & 27.692391 \\ \mathrm{H} & 36.726040 & 41.307314 & 27.708883 \\ \mathrm{H} & 39.000927 & 41.122125 & 28.337294 \\ \mathrm{H} & 39.091447 & 42.824290 & 28.732616 \\ \mathrm{H} & 40.214832 & 40.864616 & 30.907296 \\ \mathrm{H} & 37.107006 & 40.623641 & 33.836663 \\ \mathrm{H} & 35.490071 & 41.592313 & 32.279497 \\ \mathrm{H} & 34.212879 & 41.447121 & 29.194213 \\ \mathrm{O} & 34.490206 & 44.584442 & 28.873464 \\ \mathrm{H} & 30.396875 & 44.378583 & 28.852458 \\ \mathrm{H} & 31.815869 & 44.852906 & 27.921259 \\ \mathrm{H} & 30.496704 & 46.662366 & 29.106934 \\ \mathrm{C} & 30.982841 & 46.098913 & 31.028295 \\ \mathrm{O} & 29.865789 & 45.891008 & 31.371301 \\ \mathrm{H} & 32.621301 & 47.849192 & 29.532574 \\ \mathrm{H} & 31.756502 & 46.267010 & 31.725898 \\ \mathrm{H} & 45.522394 & 39.993501 & 36.648751 \\ \mathrm{H} & 41.516243 & 35.031201 & 33.805674\end{array}$

$\begin{array}{lrll}\text { C7-C10-C11-C12 130 } & & \\ \text { C } & 44.793705 & 39.850203 & 35.849841 \\ \text { C } & 45.116429 & 39.140684 & 34.700942 \\ \text { H } & 46.063076 & 38.644734 & 34.598690 \\ \text { C } & 44.222164 & 39.062010 & 33.654765 \\ \text { H } & 44.480290 & 38.529846 & 32.759099 \\ \text { C } & 42.992795 & 39.706010 & 33.732470 \\ \text { O } & 42.175018 & 39.646801 & 32.687161 \\ \text { H } & 41.249699 & 39.865814 & 32.922039 \\ \text { C } & 42.660328 & 40.402292 & 34.890616 \\ \text { H } & 41.707922 & 40.893363 & 34.947681 \\ \text { C } & 43.560134 & 40.479673 & 35.932513 \\ \text { H } & 43.287038 & 41.044985 & 36.806790 \\ \text { C } & 41.570058 & 35.869889 & 34.546012 \\ \text { H } & 41.287450 & 35.488491 & 35.519905 \\ \text { H } & 42.587277 & 36.238745 & 34.625972 \\ \text { C } & 40.660087 & 37.031411 & 34.154356 \\ \text { H } & 40.819597 & 37.858115 & 34.840696 \\ \text { H } & 40.919517 & 37.439508 & 33.183233 \\ \text { C } & 39.173917 & 36.753563 & 34.139550 \\ \text { O } & 38.694263 & 35.673840 & 34.303383 \\ \text { O } & 38.417899 & 37.799837 & 33.930245 \\ \text { H } & 38.919498 & 38.625711 & 33.771484 \\ \text { N } & 32.506471 & 46.876724 & 29.289146 \\ \text { H } & 33.340654 & 46.386818 & 29.072346\end{array}$




\begin{tabular}{|c|c|c|c|}
\hline C & 31.325877 & 46.131728 & 29.542913 \\
\hline C & 31.384575 & 44.735421 & 28.914284 \\
\hline$S$ & 32.265524 & 43.427042 & 29.836060 \\
\hline C & 33.993738 & 43.573281 & 29.347792 \\
\hline C & 34.757357 & 42.370649 & 29.643074 \\
\hline C & 36.222357 & 42.351775 & 29.677894 \\
\hline C & 36.966451 & 41.813770 & 30.735764 \\
\hline C & 36.568143 & 41.464736 & 32.053899 \\
\hline C & 37.484616 & 40.886851 & 32.899987 \\
\hline C & 38.846668 & 40.660967 & 32.530755 \\
\hline 0 & 39.656236 & 40.057747 & 33.333171 \\
\hline C & 39.226465 & 41.016606 & 31.203708 \\
\hline C & 38.309890 & 41.571091 & 30.345828 \\
\hline C & 38.473212 & 41.924620 & 28.888366 \\
\hline C & 37.029495 & 42.187715 & 28.400202 \\
\hline $\mathrm{H}$ & 36.967056 & 43.071156 & 27.774368 \\
\hline $\mathrm{H}$ & 36.648203 & 41.355172 & 27.807010 \\
\hline $\mathrm{H}$ & 38.949779 & 41.119334 & 28.341165 \\
\hline $\mathrm{H}$ & 39.086580 & 42.813296 & 28.762867 \\
\hline $\mathrm{H}$ & 40.231069 & 40.798928 & 30.883983 \\
\hline $\mathrm{H}$ & 37.180557 & 40.561139 & 33.878303 \\
\hline $\mathrm{H}$ & 35.545164 & 41.580097 & 32.360368 \\
\hline $\mathrm{H}$ & 34.270165 & 41.480466 & 29.272687 \\
\hline 0 & 34.451350 & 44.573325 & 28.854420 \\
\hline $\mathrm{H}$ & 30.380983 & 44.339693 & 28.845527 \\
\hline $\mathrm{H}$ & 31.806223 & 44.801828 & 27.920126 \\
\hline $\mathrm{H}$ & 30.461208 & 46.615352 & 29.094034 \\
\hline C & 30.949723 & 46.051378 & 31.022286 \\
\hline 0 & 29.840890 & 45.802057 & 31.367142 \\
\hline $\mathrm{H}$ & 32.571390 & 47.827894 & 29.532161 \\
\hline $\mathrm{H}$ & 31.717958 & 46.255904 & 31.714495 \\
\hline $\mathrm{H}$ & 45.538146 & 39.997147 & 36.644480 \\
\hline $\mathrm{H}$ & 41.555565 & 35.026298 & 33.821278 \\
\hline \multicolumn{4}{|c|}{ C7-C10-C11-C12 140 } \\
\hline C & 44.811921 & 39.821720 & 35.848221 \\
\hline C & 45.147940 & 39.096827 & 34.713992 \\
\hline $\mathrm{H}$ & 46.096551 & 38.600422 & 34.639372 \\
\hline C & 44.275539 & 39.012662 & 33.649150 \\
\hline $\mathrm{H}$ & 44.547356 & 38.477762 & 32.758581 \\
\hline C & 43.046058 & 39.656884 & 33.700238 \\
\hline 0 & 42.248835 & 39.588452 & 32.636537 \\
\hline $\mathrm{H}$ & 41.327731 & 39.820819 & 32.847946 \\
\hline C & 42.692394 & 40.359350 & 34.847188 \\
\hline
\end{tabular}




\begin{tabular}{|c|c|c|c|}
\hline $\mathrm{H}$ & 41.734951 & 40.843091 & 34.885400 \\
\hline C & 43.575745 & 40.447861 & 35.903670 \\
\hline $\mathrm{H}$ & 43.291947 & 41.022952 & 36.768032 \\
\hline C & 41.630805 & 35.848497 & 34.574205 \\
\hline $\mathrm{H}$ & 41.361927 & 35.466863 & 35.552595 \\
\hline $\mathrm{H}$ & 42.652297 & 36.208046 & 34.642056 \\
\hline C & 40.717857 & 37.010855 & 34.199320 \\
\hline $\mathrm{H}$ & 40.885484 & 37.837101 & 34.883518 \\
\hline $\mathrm{H}$ & 40.956094 & 37.419198 & 33.223598 \\
\hline C & 39.233799 & 36.724861 & 34.212263 \\
\hline 0 & 38.761472 & 35.653698 & 34.434342 \\
\hline 0 & 38.470499 & 37.757078 & 33.959321 \\
\hline $\mathrm{H}$ & 38.965972 & 38.574426 & 33.765740 \\
\hline $\mathrm{N}$ & 32.498237 & 46.932979 & 29.270249 \\
\hline $\mathrm{H}$ & 33.327973 & 46.438120 & 29.047252 \\
\hline$C$ & 31.313349 & 46.195501 & 29.515649 \\
\hline C & 31.367453 & 44.803501 & 28.880792 \\
\hline$S$ & 32.218808 & 43.491071 & 29.824436 \\
\hline C & 33.966707 & 43.630199 & 29.377630 \\
\hline$C$ & 34.723862 & 42.465076 & 29.783568 \\
\hline C & 36.197037 & 42.423883 & 29.764763 \\
\hline$C$ & 36.954318 & 41.817525 & 30.767759 \\
\hline C & 36.586604 & 41.446228 & 32.090812 \\
\hline C & 37.518044 & 40.845191 & 32.899914 \\
\hline C & 38.870325 & 40.616569 & 32.487252 \\
\hline 0 & 39.694944 & 40.010026 & 33.259340 \\
\hline C & 39.215386 & 40.982519 & 31.149985 \\
\hline C & 38.281250 & 41.554533 & 30.327016 \\
\hline C & 38.396943 & 41.933887 & 28.870499 \\
\hline C & 36.944817 & 42.264126 & 28.450638 \\
\hline $\mathrm{H}$ & 36.891233 & 43.165002 & 27.849899 \\
\hline $\mathrm{H}$ & 36.504300 & 41.462540 & 27.854677 \\
\hline $\mathrm{H}$ & 38.819323 & 41.123365 & 28.287339 \\
\hline $\mathrm{H}$ & 39.035959 & 42.802547 & 28.734689 \\
\hline $\mathrm{H}$ & 40.207098 & 40.752199 & 30.799911 \\
\hline $\mathrm{H}$ & 37.244951 & 40.511891 & 33.884663 \\
\hline $\mathrm{H}$ & 35.572259 & 41.568801 & 32.423421 \\
\hline $\mathrm{H}$ & 34.240079 & 41.560081 & 29.441639 \\
\hline 0 & 34.423878 & 44.610281 & 28.842661 \\
\hline $\mathrm{H}$ & 30.360626 & 44.422907 & 28.793338 \\
\hline $\mathrm{H}$ & 31.801696 & 44.865889 & 27.892853 \\
\hline $\mathrm{H}$ & 30.447878 & 46.686754 & 29.075533 \\
\hline C & 30.941434 & 46.108517 & 30.994383 \\
\hline 0 & 29.829273 & 45.876496 & 31.334837 \\
\hline
\end{tabular}




$\begin{array}{llll}\mathrm{H} & 32.567431 & 47.884661 & 29.518058 \\ \mathrm{H} & 31.715826 & 46.286234 & 31.690412 \\ \mathrm{H} & 45.545742 & 39.977651 & 36.651213 \\ \mathrm{H} & 41.596090 & 35.006581 & 33.848620\end{array}$

\begin{tabular}{|c|c|c|c|}
\hline \multicolumn{4}{|c|}{ C7-C10-C11-C12 150 } \\
\hline C & 44.834260 & 39.823634 & 35.790313 \\
\hline C & 45.203480 & 39.113525 & 34.657256 \\
\hline $\mathrm{H}$ & 46.148408 & 38.608314 & 34.606153 \\
\hline C & 44.359103 & 39.038618 & 33.569982 \\
\hline $\mathrm{H}$ & 44.656736 & 38.514064 & 32.681981 \\
\hline C & 43.135906 & 39.691080 & 33.595134 \\
\hline 0 & 42.360851 & 39.624943 & 32.506676 \\
\hline $\mathrm{H}$ & 41.455325 & 39.872566 & 32.698411 \\
\hline C & 42.754970 & 40.394204 & 34.730274 \\
\hline $\mathrm{H}$ & 41.804157 & 40.892744 & 34.746422 \\
\hline C & 43.607188 & 40.468766 & 35.813222 \\
\hline $\mathrm{H}$ & 43.297668 & 41.035171 & 36.674415 \\
\hline C & 41.745675 & 35.934516 & 34.610802 \\
\hline $\mathrm{H}$ & 41.571146 & 35.563498 & 35.613390 \\
\hline $\mathrm{H}$ & 42.773193 & 36.278265 & 34.572310 \\
\hline C & 40.814676 & 37.105920 & 34.327442 \\
\hline $\mathrm{H}$ & 41.113695 & 37.949935 & 34.942257 \\
\hline $\mathrm{H}$ & 40.888140 & 37.460599 & 33.305413 \\
\hline C & 39.362213 & 36.845667 & 34.639133 \\
\hline 0 & 38.964195 & 35.854731 & 35.158180 \\
\hline 0 & 38.531470 & 37.810210 & 34.303778 \\
\hline $\mathrm{H}$ & 38.979638 & 38.574375 & 33.935813 \\
\hline N & 32.470830 & 46.873229 & 29.258607 \\
\hline $\mathrm{H}$ & 33.270055 & 46.357701 & 28.972404 \\
\hline C & 31.259269 & 46.180583 & 29.502006 \\
\hline C & 31.283068 & 44.775337 & 28.899123 \\
\hline$S$ & 32.145852 & 43.484295 & 29.853477 \\
\hline C & 33.881731 & 43.500767 & 29.222264 \\
\hline C & 34.621938 & 42.461297 & 29.813547 \\
\hline C & 36.010045 & 42.197337 & 29.609825 \\
\hline C & 36.821667 & 41.598871 & 30.631229 \\
\hline C & 36.439544 & 41.093528 & 31.903438 \\
\hline C & 37.410626 & 40.589790 & 32.745481 \\
\hline C & 38.800846 & 40.591709 & 32.388740 \\
\hline 0 & 39.672704 & 40.075199 & 33.136133 \\
\hline C & 39.160001 & 41.119290 & 31.088519 \\
\hline C & 38.187652 & 41.605868 & 30.251204 \\
\hline C & 38.322117 & 42.150555 & 28.848339 \\
\hline
\end{tabular}




$\begin{array}{llll}\mathrm{C} & 36.883524 & 42.559217 & 28.433773 \\ \mathrm{H} & 36.820494 & 43.612975 & 28.190796 \\ \mathrm{H} & 36.564826 & 42.015526 & 27.546444 \\ \mathrm{H} & 38.716244 & 41.381945 & 28.191365 \\ \mathrm{H} & 39.007251 & 42.991722 & 28.807478 \\ \mathrm{H} & 40.198349 & 41.094259 & 30.808063 \\ \mathrm{H} & 37.145001 & 40.188340 & 33.705801 \\ \mathrm{H} & 35.407144 & 41.063154 & 32.188039 \\ \mathrm{H} & 34.137592 & 41.934168 & 30.611042 \\ \mathrm{O} & 34.225460 & 44.329236 & 28.386755 \\ \mathrm{H} & 30.266788 & 44.416432 & 28.835481 \\ \mathrm{H} & 31.705816 & 44.820751 & 27.905162 \\ \mathrm{H} & 30.409553 & 46.687580 & 29.047370 \\ \mathrm{C} & 30.881583 & 46.132787 & 30.981290 \\ \mathrm{O} & 29.759661 & 45.981151 & 31.331263 \\ \mathrm{H} & 32.572631 & 47.824203 & 29.495123 \\ \mathrm{H} & 31.668963 & 46.269089 & 31.671545 \\ \mathrm{H} & 45.552421 & 39.981748 & 36.607658 \\ \mathrm{H} & 41.624223 & 35.091439 & 33.896354\end{array}$

C7-C10-C11-C12 160

$\begin{array}{llll}\mathrm{C} & 44.839650 & 39.817630 & 35.779281 \\ \mathrm{C} & 45.217149 & 39.101381 & 34.652547 \\ \mathrm{H} & 46.162890 & 38.597645 & 34.608834 \\ \mathrm{C} & 44.381446 & 39.020279 & 33.558988 \\ \mathrm{H} & 44.683645 & 38.486644 & 32.678132 \\ \mathrm{C} & 43.158711 & 39.673083 & 33.572389 \\ \mathrm{O} & 42.389836 & 39.599261 & 32.478967 \\ \mathrm{H} & 41.484458 & 39.846681 & 32.665912 \\ \mathrm{C} & 42.771186 & 40.387412 & 34.698142 \\ \mathrm{H} & 41.821187 & 40.887846 & 34.702970 \\ \mathrm{C} & 43.613337 & 40.464861 & 35.788853 \\ \mathrm{H} & 43.297669 & 41.038045 & 36.643126 \\ \mathrm{C} & 41.762957 & 35.934506 & 34.614252 \\ \mathrm{H} & 41.591639 & 35.566525 & 35.618408 \\ \mathrm{H} & 42.789860 & 36.277735 & 34.567180 \\ \mathrm{C} & 40.826802 & 37.102189 & 34.332792 \\ \mathrm{H} & 41.122928 & 37.951669 & 34.942193 \\ \mathrm{H} & 40.888071 & 37.451484 & 33.307901 \\ \mathrm{C} & 39.378340 & 36.841238 & 34.660566 \\ \mathrm{O} & 38.985665 & 35.857561 & 35.196650 \\ \mathrm{O} & 38.542698 & 37.801548 & 34.322137 \\ \mathrm{H} & 38.988740 & 38.560003 & 33.942840 \\ \mathrm{~N} & 32.455804 & 46.865498 & 29.262844\end{array}$




\begin{tabular}{|c|c|c|c|}
\hline $\mathrm{H}$ & 33.243561 & 46.342802 & 28.959150 \\
\hline C & 31.232431 & 46.189946 & 29.506244 \\
\hline C & 31.237190 & 44.780329 & 28.910355 \\
\hline$S$ & 32.102468 & 43.485679 & 29.854955 \\
\hline C & 33.834993 & 43.508735 & 29.208025 \\
\hline C & 34.623897 & 42.568133 & 29.899540 \\
\hline C & 35.981887 & 42.260014 & 29.660124 \\
\hline C & 36.805277 & 41.615894 & 30.661565 \\
\hline C & 36.439666 & 41.094506 & 31.928652 \\
\hline C & 37.418364 & 40.568495 & 32.749037 \\
\hline C & 38.804466 & 40.573355 & 32.374957 \\
\hline 0 & 39.685371 & 40.057919 & 33.111673 \\
\hline C & 39.145445 & 41.110297 & 31.076849 \\
\hline C & 38.160239 & 41.612440 & 30.258609 \\
\hline C & 38.287722 & 42.198437 & 28.872273 \\
\hline C & 36.843427 & 42.593122 & 28.465439 \\
\hline $\mathrm{H}$ & 36.775453 & 43.637567 & 28.188613 \\
\hline $\mathrm{H}$ & 36.517434 & 42.022728 & 27.597871 \\
\hline $\mathrm{H}$ & 38.701888 & 41.461051 & 28.193479 \\
\hline $\mathrm{H}$ & 38.954814 & 43.055351 & 28.863578 \\
\hline $\mathrm{H}$ & 40.180434 & 41.093018 & 30.783940 \\
\hline $\mathrm{H}$ & 37.163863 & 40.161423 & 33.709920 \\
\hline $\mathrm{H}$ & 35.412122 & 41.067715 & 32.230885 \\
\hline $\mathrm{H}$ & 34.169725 & 42.120805 & 30.761731 \\
\hline 0 & 34.128011 & 44.242842 & 28.269226 \\
\hline $\mathrm{H}$ & 30.216564 & 44.432632 & 28.856441 \\
\hline $\mathrm{H}$ & 31.650228 & 44.816909 & 27.912101 \\
\hline $\mathrm{H}$ & 30.392702 & 46.703037 & 29.040238 \\
\hline C & 30.848265 & 46.150206 & 30.985405 \\
\hline 0 & 29.725212 & 46.000231 & 31.336542 \\
\hline $\mathrm{H}$ & 32.569208 & 47.815732 & 29.497226 \\
\hline $\mathrm{H}$ & 31.634242 & 46.281854 & 31.677193 \\
\hline $\mathrm{H}$ & 45.550269 & 39.976257 & 36.602814 \\
\hline $\mathrm{H}$ & 41.634881 & 35.090597 & 33.90172 \\
\hline \multicolumn{4}{|c|}{ C7-C10-C11-C12 170 } \\
\hline$C$ & 44.846279 & 39.801807 & 35.778128 \\
\hline C & 45.232156 & 39.078106 & 34.659283 \\
\hline $\mathrm{H}$ & 46.181939 & 38.581634 & 34.624194 \\
\hline C & 44.403363 & 38.988525 & 33.561280 \\
\hline $\mathrm{H}$ & 44.714097 & 38.450698 & 32.686228 \\
\hline C & 43.178919 & 39.638737 & 33.563138 \\
\hline 0 & 42.418086 & 39.559067 & 32.464068 \\
\hline $\mathrm{H}$ & 41.513936 & 39.816295 & 32.6412 \\
\hline
\end{tabular}




\begin{tabular}{|c|c|c|c|}
\hline C & 42.780651 & 40.353652 & 34.684554 \\
\hline $\mathrm{H}$ & 41.828807 & 40.850408 & 34.683730 \\
\hline C & 43.615248 & 40.439393 & 35.780407 \\
\hline $\mathrm{H}$ & 43.293659 & 41.017493 & 36.629314 \\
\hline C & 41.778073 & 35.920378 & 34.617719 \\
\hline $\mathrm{H}$ & 41.604076 & 35.548957 & 35.619867 \\
\hline $\mathrm{H}$ & 42.806867 & 36.257488 & 34.573065 \\
\hline C & 40.847283 & 37.094493 & 34.343798 \\
\hline $\mathrm{H}$ & 41.145096 & 37.940918 & 34.956348 \\
\hline $\mathrm{H}$ & 40.909239 & 37.451954 & 33.322223 \\
\hline C & 39.397956 & 36.835377 & 34.670705 \\
\hline 0 & 39.003862 & 35.854489 & 35.210572 \\
\hline 0 & 38.563210 & 37.795991 & 34.329797 \\
\hline $\mathrm{H}$ & 39.009725 & 38.548279 & 33.940427 \\
\hline $\mathrm{N}$ & 32.459665 & 46.869926 & 29.247220 \\
\hline $\mathrm{H}$ & 33.243174 & 46.344858 & 28.937494 \\
\hline C & 31.232504 & 46.200195 & 29.492437 \\
\hline C & 31.224944 & 44.790273 & 28.897503 \\
\hline$S$ & 32.081363 & 43.494346 & 29.846781 \\
\hline C & 33.812410 & 43.512330 & 29.196234 \\
\hline C & 34.639646 & 42.671547 & 29.970811 \\
\hline C & 35.994841 & 42.381547 & 29.735308 \\
\hline C & 36.817397 & 41.675793 & 30.702764 \\
\hline C & 36.461312 & 41.135888 & 31.963163 \\
\hline C & 37.442325 & 40.576909 & 32.758827 \\
\hline C & 38.822858 & 40.564468 & 32.362475 \\
\hline 0 & 39.708085 & 40.036033 & 33.084276 \\
\hline C & 39.149953 & 41.102826 & 31.063431 \\
\hline C & 38.160026 & 41.637762 & 30.268621 \\
\hline C & 38.278543 & 42.251094 & 28.893355 \\
\hline C & 36.831891 & 42.656124 & 28.508223 \\
\hline $\mathrm{H}$ & 36.771523 & 43.686512 & 28.184078 \\
\hline $\mathrm{H}$ & 36.475313 & 42.052390 & 27.675403 \\
\hline $\mathrm{H}$ & 38.689338 & 41.531302 & 28.194020 \\
\hline $\mathrm{H}$ & 38.945271 & 43.108545 & 28.900610 \\
\hline $\mathrm{H}$ & 40.178031 & 41.060829 & 30.749339 \\
\hline $\mathrm{H}$ & 37.195842 & 40.161666 & 33.718220 \\
\hline $\mathrm{H}$ & 35.439224 & 41.127516 & 32.284096 \\
\hline $\mathrm{H}$ & 34.196975 & 42.262664 & 30.858121 \\
\hline 0 & 34.082663 & 44.170874 & 28.194989 \\
\hline $\mathrm{H}$ & 30.200907 & 44.452707 & 28.842157 \\
\hline $\mathrm{H}$ & 31.640324 & 44.824423 & 27.900176 \\
\hline $\mathrm{H}$ & 30.396127 & 46.720588 & 29.028425 \\
\hline C & 30.851663 & 46.158489 & 30.971743 \\
\hline
\end{tabular}




$\begin{array}{llll}\mathrm{O} & 29.728183 & 46.011418 & 31.323006 \\ \mathrm{H} & 32.576870 & 47.819037 & 29.481908 \\ \mathrm{H} & 31.639093 & 46.283375 & 31.663100 \\ \mathrm{H} & 45.551631 & 39.967172 & 36.604598 \\ \mathrm{H} & 41.645614 & 35.079208 & 33.902653\end{array}$

$\begin{array}{llll}\text { C7-C10-C11-C12 180 } & & \\ \text { C } & 44.848165 & 39.789245 & 35.772246 \\ \text { C } & 45.241531 & 39.056060 & 34.661867 \\ \text { H } & 46.194932 & 38.566308 & 34.635136 \\ \text { C } & 44.420865 & 38.955618 & 33.558884 \\ \text { H } & 44.739177 & 38.410361 & 32.691196 \\ \mathrm{C} & 43.193999 & 39.601422 & 33.548524 \\ \text { O } & 42.441000 & 39.511967 & 32.444509 \\ \text { H } & 41.535771 & 39.770334 & 32.611441 \\ \mathrm{C} & 42.786424 & 40.322256 & 34.662613 \\ \text { H } & 41.832308 & 40.814918 & 34.652098 \\ \mathrm{C} & 43.613778 & 40.420160 & 35.763536 \\ \mathrm{H} & 43.283882 & 41.003171 & 36.605993 \\ \mathrm{C} & 41.775348 & 35.915116 & 34.612281 \\ \mathrm{H} & 41.602771 & 35.543608 & 35.614499 \\ \mathrm{H} & 42.803785 & 36.253482 & 34.568301 \\ \mathrm{C} & 40.843204 & 37.086307 & 34.335415 \\ \mathrm{H} & 41.141906 & 37.933373 & 34.946329 \\ \mathrm{H} & 40.909754 & 37.442979 & 33.313828 \\ \mathrm{C} & 39.394475 & 36.825768 & 34.664516 \\ \mathrm{O} & 39.004489 & 35.852529 & 35.220797 \\ \mathrm{O} & 38.555398 & 37.776827 & 34.307653 \\ \mathrm{H} & 39.000636 & 38.523174 & 33.906692 \\ \mathrm{~N} & 32.456808 & 46.861267 & 29.252390 \\ \mathrm{C} & 33.237299 & 46.331159 & 28.944515 \\ \mathrm{H} & 31.224613 & 46.201272 & 29.499138 \\ \mathrm{C} & 31.207295 & 44.792601 & 28.902740 \\ \mathrm{C} & 32.065898 & 43.499087 & 29.853460 \\ \mathrm{C} & 33.791365 & 43.511320 & 29.190764 \\ \mathrm{C} & 34.658036 & 42.778054 & 30.032476 \\ \mathrm{C} & 36.010622 & 42.501986 & 29.797204 \\ \mathrm{C} & 36.828083 & 41.732774 & 30.729498 \\ \mathrm{C} & 36.478839 & 41.174328 & 31.981571 \\ \mathrm{C} & 37.459002 & 40.579724 & 32.751979 \\ \mathrm{C} & 38.833269 & 40.547237 & 32.334083 \\ \mathrm{C} & 39.159339 & 41.670766 & 30.271309\end{array}$




$\begin{array}{llll}\text { C } & 38.275379 & 42.331131 & 28.917362 \\ \text { C } & 36.825892 & 42.733705 & 28.546260 \\ \text { H } & 36.767331 & 43.750967 & 28.184981 \\ \text { H } & 36.449955 & 42.104100 & 27.741837 \\ \text { H } & 38.699013 & 41.647481 & 28.191133 \\ \text { H } & 38.929237 & 43.197230 & 28.961893 \\ \text { H } & 40.175274 & 41.050234 & 30.713624 \\ \text { H } & 37.219478 & 40.155281 & 33.709139 \\ \text { H } & 35.463839 & 41.189892 & 32.324065 \\ \text { H } & 34.228348 & 42.401143 & 30.940284 \\ \text { O } & 34.032708 & 44.078320 & 28.127118 \\ \text { H } & 30.182760 & 44.457391 & 28.848714 \\ \text { H } & 31.621553 & 44.824972 & 27.904657 \\ \text { H } & 30.393262 & 46.731905 & 29.037745 \\ \text { C } & 30.844522 & 46.164248 & 30.979467 \\ \text { O } & 29.720854 & 46.019696 & 31.332849 \\ \text { H } & 32.577125 & 47.811701 & 29.482202 \\ \text { H } & 31.633523 & 46.287024 & 31.670142 \\ \text { H } & 45.548933 & 39.962514 & 36.601703 \\ \text { H } & 41.642875 & 35.074227 & 33.897799\end{array}$

$\begin{array}{lrll}\text { C7-C10-C11-C12 } 0^{\circ} & & \\ \mathrm{C} & 44.796536 & 39.858818 & 35.804843 \\ \mathrm{C} & 45.156267 & 39.172817 & 34.652344 \\ \mathrm{H} & 46.105640 & 38.678681 & 34.574681 \\ \mathrm{C} & 44.297111 & 39.114418 & 33.576195 \\ \mathrm{H} & 44.586928 & 38.612948 & 32.672802 \\ \mathrm{C} & 43.070423 & 39.760216 & 33.631137 \\ \mathrm{O} & 42.291412 & 39.721140 & 32.544309 \\ \mathrm{H} & 41.397394 & 40.008546 & 32.725691 \\ \mathrm{C} & 42.693696 & 40.426746 & 34.788816 \\ \mathrm{H} & 41.738197 & 40.915686 & 34.831759 \\ \mathrm{C} & 43.559953 & 40.483144 & 35.862399 \\ \mathrm{H} & 43.259763 & 41.032025 & 36.737888 \\ \mathrm{C} & 41.670666 & 35.926352 & 34.579702 \\ \mathrm{H} & 41.434507 & 35.558765 & 35.570883 \\ \mathrm{H} & 42.691932 & 36.289039 & 34.606489 \\ \mathrm{C} & 40.738160 & 37.079700 & 34.218611 \\ \mathrm{H} & 40.950534 & 37.925900 & 34.866595 \\ \mathrm{H} & 40.910293 & 37.451589 & 33.213360 \\ \mathrm{C} & 39.265053 & 36.779009 & 34.355070 \\ \mathrm{O} & 38.829779 & 35.735104 & 34.715165 \\ \mathrm{O} & 38.452196 & 37.769389 & 34.047075 \\ \mathrm{H} & 38.905567 & 38.567073 & 33.773654\end{array}$




\begin{tabular}{|c|c|c|c|}
\hline $\mathrm{N}$ & 32.653390 & 46.849868 & 29.436728 \\
\hline $\mathrm{H}$ & 33.520950 & 46.372095 & 29.389541 \\
\hline C & 31.466108 & 46.089124 & 29.654989 \\
\hline C & 31.587287 & 44.662472 & 29.076157 \\
\hline$S$ & 31.798081 & 43.295004 & 30.265011 \\
\hline C & 33.522163 & 42.647201 & 30.468077 \\
\hline C & 34.532784 & 42.910352 & 29.505750 \\
\hline C & 35.889647 & 42.548119 & 29.547835 \\
\hline C & 36.681344 & 41.808527 & 30.557276 \\
\hline C & 36.296778 & 41.043604 & 31.679146 \\
\hline C & 37.270466 & 40.527813 & 32.521028 \\
\hline C & 38.670856 & 40.718889 & 32.283256 \\
\hline 0 & 39.542186 & 40.201803 & 33.033201 \\
\hline C & 39.039444 & 41.457274 & 31.102688 \\
\hline C & 38.059688 & 41.991209 & 30.287143 \\
\hline C & 38.250727 & 42.848436 & 29.059778 \\
\hline C & 36.846757 & 42.907874 & 28.431579 \\
\hline $\mathrm{H}$ & 36.635233 & 43.882948 & 28.002936 \\
\hline $\mathrm{H}$ & 36.773190 & 42.173674 & 27.626120 \\
\hline $\mathrm{H}$ & 38.992501 & 42.441786 & 28.384370 \\
\hline $\mathrm{H}$ & 38.587006 & 43.842503 & 29.338724 \\
\hline $\mathrm{H}$ & 40.086540 & 41.602282 & 30.901100 \\
\hline $\mathrm{H}$ & 36.981854 & 39.966003 & 33.389275 \\
\hline $\mathrm{H}$ & 35.264848 & 40.868414 & 31.879915 \\
\hline $\mathrm{H}$ & 34.239723 & 43.521174 & 28.670250 \\
\hline 0 & 33.532892 & 41.931533 & 31.454590 \\
\hline $\mathrm{H}$ & 30.654129 & 44.423485 & 28.585524 \\
\hline $\mathrm{H}$ & 32.349892 & 44.649332 & 28.310209 \\
\hline $\mathrm{H}$ & 30.635937 & 46.561761 & 29.138393 \\
\hline C & 31.025560 & 46.105475 & 31.122290 \\
\hline 0 & 29.885025 & 46.010312 & 31.443105 \\
\hline $\mathrm{H}$ & 32.669247 & 47.815283 & 29.631463 \\
\hline $\mathrm{H}$ & 31.794850 & 46.248925 & 31.830081 \\
\hline $\mathrm{H}$ & 45.526838 & 40.014931 & 36.610898 \\
\hline $\mathrm{H}$ & 41.613430 & 35.074283 & 33.867260 \\
\hline \multicolumn{4}{|c|}{ C7-C10-C11-C12-10 } \\
\hline C & 44.807040 & 39.859867 & 35.815726 \\
\hline C & 45.160340 & 39.173745 & 34.661378 \\
\hline $\mathrm{H}$ & 46.106821 & 38.674138 & 34.581829 \\
\hline C & 44.296223 & 39.117699 & 33.589212 \\
\hline $\mathrm{H}$ & 44.579613 & 38.612280 & 32.685960 \\
\hline C & 43.071200 & 39.766479 & 33.649820 \\
\hline 0 & 42.286693 & 39.729998 & 32.566909 \\
\hline
\end{tabular}




$\begin{array}{llll}\text { H } & 41.394034 & 40.019447 & 32.752632 \\ \text { C } & 42.701071 & 40.432236 & 34.809988 \\ \text { H } & 41.747127 & 40.923544 & 34.858558 \\ \text { C } & 43.572257 & 40.486928 & 35.879478 \\ \text { H } & 43.276166 & 41.035214 & 36.756745 \\ \text { C } & 41.657165 & 35.932463 & 34.582145 \\ \text { H } & 41.404502 & 35.565710 & 35.569487 \\ \text { H } & 42.677050 & 36.297281 & 34.626018 \\ \text { C } & 40.725980 & 37.083105 & 34.208506 \\ \text { H } & 40.924520 & 37.926760 & 34.864097 \\ \text { H } & 40.912420 & 37.462200 & 33.208353 \\ \text { C } & 39.250875 & 36.777279 & 34.314670 \\ \text { O } & 38.809839 & 35.723414 & 34.636776 \\ \text { O } & 38.442253 & 37.776756 & 34.025135 \\ \text { H } & 38.899345 & 38.580937 & 33.778596 \\ \text { N } & 32.671244 & 46.801111 & 29.387377 \\ \text { H } & 33.536998 & 46.317553 & 29.364362 \\ \text { C } & 31.477044 & 46.057155 & 29.627086 \\ \text { C } & 31.570876 & 44.625000 & 29.062614 \\ \text { S } & 31.782519 & 43.268542 & 30.263199 \\ \text { C } & 33.496351 & 42.597745 & 30.454147 \\ \text { H } & 34.526519 & 42.902698 & 29.528383 \\ \text { C } & 35.625527 & 44.388840 & 28.595868 \\ \text { C } & 35.870479 & 42.485387 & 29.548244 \\ \text { C } & 36.667816 & 41.794459 & 30.586431 \\ \text { C } & 36.288435 & 41.104600 & 31.759708 \\ \text { C } & 37.266241 & 40.591371 & 32.597334 \\ \text { C } & 38.665512 & 40.735666 & 32.323119 \\ \text { O } & 39.540636 & 40.222701 & 33.071636 \\ \text { C } & 39.027060 & 41.424431 & 31.111575 \\ \text { C } & 38.044042 & 41.947513 & 30.292561 \\ \text { C } & 38.237156 & 42.746024 & 29.027110 \\ \text { C } & 36.822773 & 42.835441 & 28.424370 \\ \text { H } & 36.623446 & 43.820436 & 28.012255 \\ \text { H } & 36.719815 & 42.115349 & 27.610302 \\ \text { H } & 38.944051 & 42.278912 & 28.353638 \\ \text { H } & 38.625605 & 43.734821 & 29.252913 \\ \text { H } & 36.073047 & 41.542580 & 30.887727 \\ \text { H } & & 46.538318 & 29.109013\end{array}$




$\begin{array}{llll}\text { C } & 31.042428 & 46.091426 & 31.097474 \\ \text { O } & 29.900560 & 46.029535 & 31.418972 \\ \text { H } & 32.690007 & 47.768670 & 29.569900 \\ \text { H } & 31.815000 & 46.221580 & 31.804016 \\ \text { H } & 45.541184 & 40.013622 & 36.619092 \\ \text { H } & 41.613205 & 35.078803 & 33.870695\end{array}$

$\begin{array}{llll}\text { C7-C10-C11-C12 -20 } & & \\ \mathrm{C} & 44.797604 & 39.870793 & 35.844559 \\ \mathrm{C} & 45.138736 & 39.166426 & 34.697258 \\ \mathrm{H} & 46.082156 & 38.660198 & 34.617525 \\ \mathrm{C} & 44.268029 & 39.105000 & 33.630707 \\ \mathrm{H} & 44.542864 & 38.585047 & 32.732978 \\ \mathrm{C} & 43.047152 & 39.762919 & 33.689287 \\ \mathrm{O} & 42.256294 & 39.720549 & 32.612737 \\ \mathrm{H} & 41.360143 & 40.006266 & 32.801078 \\ \mathrm{C} & 42.690413 & 40.447287 & 34.843289 \\ \mathrm{H} & 41.741672 & 40.948377 & 34.888772 \\ \mathrm{C} & 43.568168 & 40.508961 & 35.906943 \\ \mathrm{H} & 43.280147 & 41.068659 & 36.779259 \\ \mathrm{C} & 41.604616 & 35.930678 & 34.551926 \\ \mathrm{H} & 41.325371 & 35.561873 & 35.531915 \\ \mathrm{H} & 42.623197 & 36.297443 & 34.621333 \\ \mathrm{C} & 40.684688 & 37.083533 & 34.154050 \\ \mathrm{H} & 40.854888 & 37.918432 & 34.827951 \\ \mathrm{H} & 40.919007 & 37.476806 & 33.169333 \\ \mathrm{C} & 39.202782 & 36.787214 & 34.190024 \\ \mathrm{O} & 38.738836 & 35.719518 & 34.425676 \\ \mathrm{O} & 38.414146 & 37.812040 & 33.940593 \\ \mathrm{H} & 38.882441 & 38.627135 & 33.752185 \\ \mathrm{~N} & 32.695754 & 46.795137 & 29.403874 \\ \mathrm{H} & 33.564364 & 46.318294 & 29.370893 \\ \mathrm{C} & 31.501300 & 46.047819 & 29.630342 \\ \mathrm{C} & 31.603271 & 44.613209 & 29.082413 \\ \mathrm{C} & 31.882996 & 43.298678 & 30.318621 \\ \mathrm{C} & 33.575059 & 42.569742 & 30.380447 \\ \mathrm{C} & 34.613127 & 42.948419 & 29.498789 \\ \mathrm{C} & 35.969881 & 42.502470 & 29.546723 \\ \mathrm{C} & 36.742615 & 41.912342 & 30.628046 \\ \mathrm{C} & 36.388888 & 41.550400 & 31.960823 \\ \mathrm{C} & 37.339621 & 40.976548 & 32.785385 \\ \mathrm{C} & 39.558916 & 40.264607 & 33.145661 \\ \mathrm{C} & & & \\ \mathrm{C} & 41.057226 & 41.247907 & 31.061556\end{array}$




$\begin{array}{llll}\text { C } & 38.099753 & 41.778769 & 30.226401 \\ \text { C } & 38.289446 & 42.247421 & 28.805736 \\ \text { C } & 36.885477 & 42.706355 & 28.358001 \\ \text { H } & 36.893260 & 43.747145 & 28.036618 \\ \text { H } & 36.540565 & 42.117878 & 27.509824 \\ \text { H } & 38.656024 & 41.438873 & 28.182182 \\ \text { H } & 39.017552 & 43.051001 & 28.745070 \\ \text { H } & 40.080002 & 41.130323 & 30.746930 \\ \text { H } & 37.073599 & 40.674719 & 33.781435 \\ \text { H } & 35.382020 & 41.654116 & 32.297248 \\ \text { H } & 34.364865 & 43.648567 & 28.720808 \\ \text { O } & 33.566512 & 41.709104 & 31.240845 \\ \text { H } & 30.644772 & 44.347129 & 28.660427 \\ \text { H } & 32.326517 & 44.573700 & 28.279554 \\ \text { H } & 30.681945 & 46.530500 & 29.106065 \\ \text { C } & 31.056999 & 46.074846 & 31.100189 \\ \text { O } & 29.916242 & 45.986806 & 31.417773 \\ \text { H } & 32.703124 & 47.764748 & 29.579770 \\ \text { H } & 31.823780 & 46.218106 & 31.810761 \\ \text { H } & 45.536863 & 40.026828 & 36.643323 \\ \text { H } & 41.582066 & 35.078734 & 33.837951\end{array}$

$\begin{array}{lrrr}\text { C7-C10-C11-C12-30 } & & \\ \mathrm{C} & 44.721587 & 39.870832 & 35.835384 \\ \mathrm{C} & 45.015341 & 39.136081 & 34.693998 \\ \mathrm{H} & 45.948867 & 38.613713 & 34.596359 \\ \mathrm{C} & 44.108575 & 39.063930 & 33.656855 \\ \mathrm{H} & 44.346299 & 38.517868 & 32.763953 \\ \mathrm{C} & 42.899406 & 39.742982 & 33.735767 \\ \mathrm{O} & 42.077924 & 39.688023 & 32.687129 \\ \mathrm{H} & 41.175481 & 39.966217 & 32.885241 \\ \mathrm{C} & 42.590269 & 40.458200 & 34.885470 \\ \mathrm{H} & 41.648403 & 40.970446 & 34.944509 \\ \mathrm{C} & 43.503572 & 40.528216 & 35.918663 \\ \mathrm{H} & 43.254114 & 41.108502 & 36.789280 \\ \mathrm{C} & 41.525251 & 35.940350 & 34.542321 \\ \mathrm{H} & 41.248380 & 35.561617 & 35.519737 \\ \mathrm{H} & 42.539662 & 36.316727 & 34.624004 \\ \mathrm{C} & 40.593526 & 37.086013 & 34.141589 \\ \mathrm{H} & 40.758411 & 37.924879 & 34.811452 \\ \mathrm{H} & 40.826128 & 37.478301 & 33.156493 \\ \mathrm{C} & 39.113943 & 36.780541 & 34.177286 \\ \mathrm{O} & 38.663054 & 35.705961 & 34.418963 \\ \mathrm{O} & 38.319968 & 37.792323 & 33.923778\end{array}$




\begin{tabular}{|c|c|c|c|}
\hline $\mathrm{H}$ & 38.768017 & 38.623022 & 33.710246 \\
\hline $\mathrm{N}$ & 32.709841 & 46.778237 & 29.436837 \\
\hline $\mathrm{H}$ & 33.590363 & 46.328558 & 29.392107 \\
\hline C & 31.554784 & 45.992084 & 29.703189 \\
\hline C & 31.718060 & 44.539589 & 29.214699 \\
\hline$S$ & 32.177969 & 43.276286 & 30.475449 \\
\hline C & 33.798879 & 42.547739 & 30.027402 \\
\hline C & 34.839900 & 43.343380 & 29.476130 \\
\hline C & 36.263625 & 42.943729 & 29.567942 \\
\hline C & 36.866293 & 42.195134 & 30.575547 \\
\hline C & 36.403579 & 41.797131 & 31.879502 \\
\hline C & 37.262419 & 41.101747 & 32.719611 \\
\hline C & 38.615482 & 40.804240 & 32.383889 \\
\hline 0 & 39.398343 & 40.182874 & 33.168140 \\
\hline C & 39.059717 & 41.209481 & 31.066661 \\
\hline C & 38.223345 & 41.874638 & 30.222512 \\
\hline C & 38.500651 & 42.367115 & 28.824446 \\
\hline C & 37.138682 & 42.896279 & 28.328768 \\
\hline $\mathrm{H}$ & 37.234506 & 43.868850 & 27.854625 \\
\hline $\mathrm{H}$ & 36.696683 & 42.225306 & 27.591637 \\
\hline $\mathrm{H}$ & 38.883202 & 41.568496 & 28.199538 \\
\hline $\mathrm{H}$ & 39.244150 & 43.158941 & 28.828577 \\
\hline $\mathrm{H}$ & 40.067319 & 40.960624 & 30.780036 \\
\hline $\mathrm{H}$ & 36.919396 & 40.784636 & 33.687174 \\
\hline $\mathrm{H}$ & 35.403425 & 42.006413 & 32.196505 \\
\hline $\mathrm{H}$ & 34.647155 & 44.401764 & 29.438162 \\
\hline 0 & 33.890396 & 41.376993 & 30.291212 \\
\hline $\mathrm{H}$ & 30.763232 & 44.204655 & 28.843078 \\
\hline $\mathrm{H}$ & 32.417459 & 44.504593 & 28.390781 \\
\hline $\mathrm{H}$ & 30.703563 & 46.400621 & 29.169585 \\
\hline C & 31.110374 & 46.034509 & 31.167823 \\
\hline 0 & 29.972793 & 45.897192 & 31.487648 \\
\hline $\mathrm{H}$ & 32.701745 & 47.750021 & 29.613536 \\
\hline $\mathrm{H}$ & 31.868178 & 46.218861 & 31.882758 \\
\hline $\mathrm{H}$ & 45.481574 & 40.024883 & 36.615587 \\
\hline $\mathrm{H}$ & 41.518697 & 35.090257 & 33.826319 \\
\hline \multicolumn{4}{|c|}{$\mathrm{C} 7-\mathrm{C} 10-\mathrm{C} 11-\mathrm{C} 12-40^{\circ}$} \\
\hline C & 44.691129 & 39.902183 & 35.813880 \\
\hline C & 45.000360 & 39.194459 & 34.658816 \\
\hline $\mathrm{H}$ & 45.945355 & 38.694407 & 34.550288 \\
\hline C & 44.096180 & 39.125747 & 33.620488 \\
\hline $\mathrm{H}$ & 44.346373 & 38.598256 & 32.719803 \\
\hline C & 42.871907 & 39.779307 & 33.708250 \\
\hline
\end{tabular}




\begin{tabular}{|c|c|c|c|}
\hline 0 & 42.052867 & 39.724781 & 32.657430 \\
\hline$H$ & 41.142466 & 39.975637 & 32.861989 \\
\hline 0 & 42.551382 & 40.468652 & 34.870767 \\
\hline $\mathrm{H}$ & 41.602382 & 40.966093 & 34.943941 \\
\hline C & 43.461198 & 40.536174 & 35.907933 \\
\hline $\mathrm{H}$ & 43.195245 & 41.098030 & 36.786432 \\
\hline$C$ & 41.509013 & 35.949882 & 34.519776 \\
\hline $\mathrm{H}$ & 41.226396 & 35.583567 & 35.500325 \\
\hline $\mathrm{H}$ & 42.520930 & 36.333594 & 34.593665 \\
\hline C & 40.581169 & 37.092554 & 34.104515 \\
\hline $\mathrm{H}$ & 40.735443 & 37.932510 & 34.776078 \\
\hline $\mathrm{H}$ & 40.826671 & 37.485271 & 33.123639 \\
\hline C & 39.099550 & 36.789662 & 34.124882 \\
\hline 0 & 38.646240 & 35.713319 & 34.356603 \\
\hline 0 & 38.309081 & 37.803576 & 33.869092 \\
\hline $\mathrm{H}$ & 38.756272 & 38.638601 & 33.660470 \\
\hline $\mathrm{N}$ & 32.734297 & 46.760330 & 29.419715 \\
\hline $\mathrm{H}$ & 33.610597 & 46.318292 & 29.296579 \\
\hline C & 31.574741 & 45.968925 & 29.679941 \\
\hline C & 31.716203 & 44.532664 & 29.147392 \\
\hline$S$ & 32.168844 & 43.233251 & 30.368965 \\
\hline C & 33.764440 & 42.529596 & 29.812565 \\
\hline C & 34.847436 & 43.402027 & 29.479190 \\
\hline C & 36.270621 & 42.983440 & 29.554129 \\
\hline C & 36.858680 & 42.195353 & 30.542326 \\
\hline C & 36.371096 & 41.716767 & 31.798019 \\
\hline C & 37.215890 & 41.020076 & 32.633579 \\
\hline C & 38.596829 & 40.793192 & 32.334672 \\
\hline 0 & 39.372982 & 40.174209 & 33.131165 \\
\hline C & 39.061115 & 41.249956 & 31.050149 \\
\hline C & 38.226555 & 41.921265 & 30.207543 \\
\hline C & 38.522514 & 42.473054 & 28.835222 \\
\hline C & 37.147633 & 42.942400 & 28.315623 \\
\hline $\mathrm{H}$ & 37.209761 & 43.908187 & 27.823356 \\
\hline $\mathrm{H}$ & 36.739131 & 42.239712 & 27.587980 \\
\hline $\mathrm{H}$ & 38.971675 & 41.722468 & 28.196738 \\
\hline $\mathrm{H}$ & 39.216261 & 43.306749 & 28.888820 \\
\hline $\mathrm{H}$ & 40.084431 & 41.046947 & 30.782498 \\
\hline $\mathrm{H}$ & 36.848256 & 40.642346 & 33.570749 \\
\hline $\mathrm{H}$ & 35.341697 & 41.848799 & 32.069156 \\
\hline $\mathrm{H}$ & 34.657733 & 44.434057 & 29.723753 \\
\hline 0 & 33.827677 & 41.319893 & 29.825413 \\
\hline $\mathrm{H}$ & 30.758913 & 44.222430 & 28.762428 \\
\hline $\mathrm{H}$ & 32.415910 & 44.520065 & 28.323679 \\
\hline
\end{tabular}




$\begin{array}{llll}\text { H } & 30.726296 & 46.408214 & 29.164290 \\ \mathrm{C} & 31.147060 & 45.982189 & 31.151729 \\ \mathrm{O} & 30.018648 & 45.806579 & 31.471645 \\ \mathrm{H} & 32.724619 & 47.726386 & 29.611958 \\ \mathrm{H} & 31.902317 & 46.184515 & 31.857244 \\ \mathrm{H} & 45.450385 & 40.054880 & 36.595080 \\ \mathrm{H} & 41.506363 & 35.092395 & 33.811558\end{array}$

$\begin{array}{llll}\text { C7-C10-C11-C12-50 } & & \\ \mathrm{C} & 44.708093 & 39.937743 & 35.820368 \\ \mathrm{C} & 45.014554 & 39.240788 & 34.658062 \\ \mathrm{H} & 45.958647 & 38.740949 & 34.542006 \\ \mathrm{C} & 44.107404 & 39.182448 & 33.621682 \\ \mathrm{H} & 44.354510 & 38.661089 & 32.716717 \\ \mathrm{C} & 42.881364 & 39.832452 & 33.718842 \\ \mathrm{O} & 42.058019 & 39.785156 & 32.673162 \\ \mathrm{H} & 41.138367 & 40.009061 & 32.886693 \\ \mathrm{C} & 42.566240 & 40.514113 & 34.887981 \\ \mathrm{H} & 41.617658 & 41.010759 & 34.967788 \\ \mathrm{C} & 43.478216 & 40.570959 & 35.923543 \\ \mathrm{H} & 43.215814 & 41.128374 & 36.805509 \\ \mathrm{C} & 41.565221 & 35.987993 & 34.542539 \\ \mathrm{H} & 41.306827 & 35.620042 & 35.528473 \\ \mathrm{H} & 42.582523 & 36.361421 & 34.588158 \\ \mathrm{C} & 40.635237 & 37.138430 & 34.156661 \\ \mathrm{H} & 40.822671 & 37.980934 & 34.816168 \\ \mathrm{H} & 40.845836 & 37.523753 & 33.164858 \\ \mathrm{C} & 39.154159 & 36.846668 & 34.241708 \\ \mathrm{O} & 38.712596 & 35.782129 & 34.544769 \\ \mathrm{O} & 38.357006 & 37.847253 & 33.965428 \\ \mathrm{H} & 38.799170 & 38.678813 & 33.713307 \\ \mathrm{~N} & 32.744431 & 46.736209 & 29.407940 \\ \mathrm{H} & 33.616409 & 46.288346 & 29.274078 \\ \mathrm{C} & 31.579646 & 45.952802 & 29.669537 \\ \mathrm{C} & 31.704940 & 44.517849 & 29.129300 \\ \mathrm{~S} & 32.164288 & 43.212352 & 30.342915 \\ \mathrm{C} & 33.758235 & 42.545037 & 29.748505 \\ \mathrm{C} & 34.871508 & 43.441688 & 29.577537 \\ \mathrm{C} & 36.278983 & 42.998013 & 29.615803 \\ \mathrm{C} & 36.875079 & 42.205463 & 30.602151 \\ \mathrm{C} & 36.400394 & 41.733255 & 31.859429 \\ \mathrm{C} & 37.251765 & 41.033640 & 32.688134 \\ & 38.627339 & 40.798905 & 32.374902 \\ \mathrm{C} & 39.409588 & 40.170941 & 33.170119\end{array}$




\begin{tabular}{|c|c|c|c|}
\hline C & 39.073714 & 41.242342 & 31.086192 \\
\hline C & 38.229676 & 41.916099 & 30.249936 \\
\hline C & 38.510343 & 42.445914 & 28.865872 \\
\hline C & 37.131824 & 42.925150 & 28.362248 \\
\hline $\mathrm{H}$ & 37.196697 & 43.883448 & 27.856271 \\
\hline $\mathrm{H}$ & 36.703956 & 42.218618 & 27.650001 \\
\hline $\mathrm{H}$ & 38.935957 & 41.676561 & 28.233290 \\
\hline $\mathrm{H}$ & 39.216861 & 43.270362 & 28.893887 \\
\hline $\mathrm{H}$ & 40.090982 & 41.031346 & 30.801877 \\
\hline $\mathrm{H}$ & 36.890371 & 40.658519 & 33.628932 \\
\hline $\mathrm{H}$ & 35.373323 & 41.870601 & 32.142597 \\
\hline $\mathrm{H}$ & 34.682226 & 44.426595 & 29.970820 \\
\hline 0 & 33.814600 & 41.349860 & 29.581097 \\
\hline $\mathrm{H}$ & 30.746284 & 44.200256 & 28.748887 \\
\hline $\mathrm{H}$ & 32.404841 & 44.504617 & 28.304817 \\
\hline $\mathrm{H}$ & 30.733048 & 46.402248 & 29.159912 \\
\hline C & 31.155823 & 45.965698 & 31.142420 \\
\hline 0 & 30.028607 & 45.785635 & 31.462957 \\
\hline $\mathrm{H}$ & 32.743672 & 47.702291 & 29.601082 \\
\hline $\mathrm{H}$ & 31.911619 & 46.175649 & 31.845562 \\
\hline $\mathrm{H}$ & 45.466441 & 40.077352 & 36.604613 \\
\hline $\mathrm{H}$ & 41.533843 & 35.132903 & 33.831231 \\
\hline \multicolumn{4}{|c|}{$\mathrm{C} 7-\mathrm{C} 10-\mathrm{C} 11-\mathrm{C} 12-60^{\circ}$} \\
\hline C & 44.711097 & 39.941962 & 35.814047 \\
\hline C & 45.026291 & 39.252044 & 34.650344 \\
\hline $\mathrm{H}$ & 45.973899 & 38.758482 & 34.537859 \\
\hline C & 44.123722 & 39.192030 & 33.610234 \\
\hline $\mathrm{H}$ & 44.377306 & 38.673790 & 32.705325 \\
\hline C & 42.894768 & 39.837778 & 33.703585 \\
\hline 0 & 42.074881 & 39.788384 & 32.657098 \\
\hline $\mathrm{H}$ & 41.151875 & 40.009537 & 32.872803 \\
\hline C & 42.572539 & 40.515970 & 34.873898 \\
\hline $\mathrm{H}$ & 41.622021 & 41.009274 & 34.949110 \\
\hline C & 43.479622 & 40.573243 & 35.913117 \\
\hline $\mathrm{H}$ & 43.213163 & 41.127990 & 36.795558 \\
\hline C & 41.586696 & 35.992312 & 34.554320 \\
\hline $\mathrm{H}$ & 41.329495 & 35.629651 & 35.542418 \\
\hline $\mathrm{H}$ & 42.606193 & 36.359903 & 34.594966 \\
\hline C & 40.662849 & 37.146864 & 34.166788 \\
\hline $\mathrm{H}$ & 40.855287 & 37.988583 & 34.825620 \\
\hline $\mathrm{H}$ & 40.879771 & 37.530807 & 33.175803 \\
\hline C & 39.178741 & 36.864848 & 34.248706 \\
\hline 0 & 38.730295 & 35.804663 & 34.561430 \\
\hline
\end{tabular}




\begin{tabular}{|c|c|c|c|}
\hline 0 & 38.390113 & 37.867087 & 33.961466 \\
\hline $\mathrm{H}$ & 38.838625 & 38.699605 & 33.708384 \\
\hline $\mathrm{N}$ & 32.738951 & 46.735027 & 29.409203 \\
\hline $\mathrm{H}$ & 33.610151 & 46.288652 & 29.265075 \\
\hline C & 31.573948 & 45.953535 & 29.672452 \\
\hline C & 31.688094 & 44.524463 & 29.120354 \\
\hline S & 32.168476 & 43.214298 & 30.322629 \\
\hline C & 33.747673 & 42.568153 & 29.674736 \\
\hline C & 34.892954 & 43.452545 & 29.638748 \\
\hline C & 36.282564 & 42.976693 & 29.630166 \\
\hline C & 36.892785 & 42.194882 & 30.622936 \\
\hline C & 36.432404 & 41.733945 & 31.885539 \\
\hline C & 37.292991 & 41.037082 & 32.709452 \\
\hline C & 38.662206 & 40.797353 & 32.384170 \\
\hline 0 & 39.452505 & 40.170725 & 33.183631 \\
\hline C & 39.093027 & 41.230483 & 31.090922 \\
\hline C & 38.236858 & 41.897093 & 30.255605 \\
\hline C & 38.505241 & 42.413507 & 28.863058 \\
\hline C & 37.127106 & 42.909287 & 28.371840 \\
\hline $\mathrm{H}$ & 37.196030 & 43.871346 & 27.872639 \\
\hline $\mathrm{H}$ & 36.691541 & 42.210252 & 27.657576 \\
\hline $\mathrm{H}$ & 38.907387 & 41.632752 & 28.228483 \\
\hline $\mathrm{H}$ & 39.226696 & 43.225605 & 28.874389 \\
\hline $\mathrm{H}$ & 40.107848 & 41.020787 & 30.796836 \\
\hline $\mathrm{H}$ & 36.941337 & 40.672568 & 33.658070 \\
\hline $\mathrm{H}$ & 35.407203 & 41.873063 & 32.178296 \\
\hline $\mathrm{H}$ & 34.709847 & 44.412461 & 30.090090 \\
\hline 0 & 33.783336 & 41.408294 & 29.344996 \\
\hline $\mathrm{H}$ & 30.725063 & 44.203453 & 28.755107 \\
\hline $\mathrm{H}$ & 32.370163 & 44.509023 & 28.280886 \\
\hline $\mathrm{H}$ & 30.725464 & 46.407463 & 29.169660 \\
\hline C & 31.158430 & 45.951745 & 31.147932 \\
\hline 0 & 30.035758 & 45.757736 & 31.472976 \\
\hline $\mathrm{H}$ & 32.737626 & 47.699336 & 29.609867 \\
\hline $\mathrm{H}$ & 31.915775 & 46.164614 & 31.848401 \\
\hline $\mathrm{H}$ & 45.465843 & 40.081584 & 36.601559 \\
\hline $\mathrm{H}$ & 41.547229 & 35.134862 & 33.846731 \\
\hline \multicolumn{4}{|c|}{ C7-C10-C11-C12 -70 } \\
\hline C & 44.705454 & 39.917035 & 35.815178 \\
\hline C & 45.019069 & 39.226809 & 34.651117 \\
\hline $\mathrm{H}$ & 45.968115 & 38.736812 & 34.535322 \\
\hline C & 44.113626 & 39.160018 & 33.614000 \\
\hline $\mathrm{H}$ & 44.367345 & 38.644283 & 32.707476 \\
\hline
\end{tabular}




\begin{tabular}{|c|c|c|c|}
\hline C & 42.880955 & 39.799326 & 33.709213 \\
\hline 0 & 42.062118 & 39.747892 & 32.663666 \\
\hline $\mathrm{H}$ & 41.133385 & 39.962623 & 32.874259 \\
\hline$C$ & 42.558106 & 40.474865 & 34.881301 \\
\hline $\mathrm{H}$ & 41.604323 & 40.961674 & 34.957904 \\
\hline$C$ & 43.469992 & 40.540329 & 35.916281 \\
\hline $\mathrm{H}$ & 43.201289 & 41.090069 & 36.801698 \\
\hline C & 41.519328 & 35.949664 & 34.526690 \\
\hline $\mathrm{H}$ & 41.227303 & 35.587150 & 35.505592 \\
\hline $\mathrm{H}$ & 42.533507 & 36.326093 & 34.607785 \\
\hline C & 40.602101 & 37.097328 & 34.101872 \\
\hline $\mathrm{H}$ & 40.748457 & 37.936709 & 34.775622 \\
\hline $\mathrm{H}$ & 40.864777 & 37.493350 & 33.126617 \\
\hline C & 39.116963 & 36.805666 & 34.099070 \\
\hline 0 & 38.654811 & 35.730558 & 34.333553 \\
\hline 0 & 38.344949 & 37.820480 & 33.819249 \\
\hline $\mathrm{H}$ & 38.810579 & 38.662620 & 33.613745 \\
\hline $\mathrm{N}$ & 32.736516 & 46.709010 & 29.377412 \\
\hline $\mathrm{H}$ & 33.602508 & 46.242401 & 29.269119 \\
\hline C & 31.549321 & 45.957966 & 29.642416 \\
\hline C & 31.595766 & 44.522728 & 29.099141 \\
\hline$S$ & 32.174975 & 43.220219 & 30.271463 \\
\hline$C$ & 33.736533 & 42.661178 & 29.505544 \\
\hline C & 34.908748 & 43.493141 & 29.692331 \\
\hline C & 36.284461 & 42.996964 & 29.666025 \\
\hline C & 36.890076 & 42.180643 & 30.637471 \\
\hline C & 36.426355 & 41.685597 & 31.885046 \\
\hline C & 37.289859 & 40.978415 & 32.698018 \\
\hline C & 38.661301 & 40.755251 & 32.373023 \\
\hline 0 & 39.452402 & 40.106725 & 33.164780 \\
\hline C & 39.098851 & 41.234420 & 31.103337 \\
\hline C & 38.239600 & 41.913992 & 30.276313 \\
\hline C & 38.525507 & 42.496132 & 28.912712 \\
\hline C & 37.159740 & 43.030433 & 28.429145 \\
\hline $\mathrm{H}$ & 37.236246 & 44.027649 & 28.003295 \\
\hline $\mathrm{H}$ & 36.743199 & 42.384543 & 27.657187 \\
\hline $\mathrm{H}$ & 38.920387 & 41.743745 & 28.241011 \\
\hline $\mathrm{H}$ & 39.259313 & 43.294470 & 28.967881 \\
\hline $\mathrm{H}$ & 40.118596 & 41.047222 & 30.811627 \\
\hline $\mathrm{H}$ & 36.933494 & 40.583831 & 33.632894 \\
\hline $\mathrm{H}$ & 35.401665 & 41.817338 & 32.184160 \\
\hline $\mathrm{H}$ & 34.713284 & 44.416362 & 30.207312 \\
\hline 0 & 33.741220 & 41.625852 & 28.886745 \\
\hline $\mathrm{H}$ & 30.593130 & 44.219180 & 28.842236 \\
\hline
\end{tabular}




$\begin{array}{llll}\mathrm{H} & 32.193705 & 44.489142 & 28.198664 \\ \mathrm{H} & 30.714785 & 46.438943 & 29.141335 \\ \mathrm{C} & 31.129215 & 45.965291 & 31.117611 \\ \mathrm{O} & 30.005035 & 45.776396 & 31.441871 \\ \mathrm{H} & 32.749171 & 47.676212 & 29.567084 \\ \mathrm{H} & 31.885728 & 46.165926 & 31.822274 \\ \mathrm{H} & 45.463744 & 40.066191 & 36.598065 \\ \mathrm{H} & 41.516302 & 35.090358 & 33.820495\end{array}$

$\begin{array}{llll}\text { C7-C10-C11-C12 -80 } & & \\ \text { C } & 44.673143 & 39.920424 & 35.793682 \\ \text { C } & 44.980030 & 39.231643 & 34.626323 \\ \text { H } & 45.924592 & 38.733281 & 34.504812 \\ \text { C } & 44.071540 & 39.178136 & 33.590450 \\ \text { H } & 44.319463 & 38.661915 & 32.682621 \\ \text { C } & 42.841665 & 39.823075 & 33.689977 \\ \text { O } & 42.019910 & 39.780927 & 32.646392 \\ \text { H } & 41.087785 & 39.987168 & 32.860054 \\ \text { C } & 42.525265 & 40.492950 & 34.867166 \\ \text { H } & 41.571982 & 40.979409 & 34.952328 \\ \text { C } & 43.439870 & 40.548003 & 35.900087 \\ \text { H } & 43.175214 & 41.093641 & 36.789280 \\ \text { C } & 41.529395 & 35.968090 & 34.528205 \\ \text { H } & 41.254861 & 35.611120 & 35.514027 \\ \text { H } & 42.549184 & 36.334387 & 34.586930 \\ \text { C } & 40.615003 & 37.122084 & 34.116137 \\ \text { H } & 40.785923 & 37.962429 & 34.781914 \\ \text { H } & 40.860643 & 37.509288 & 33.132785 \\ \text { C } & 39.128035 & 36.842181 & 34.145284 \\ \text { O } & 38.669250 & 35.776676 & 34.428937 \\ \text { O } & 38.353284 & 37.847698 & 33.844191 \\ \text { H } & 38.811536 & 38.690264 & 33.612549 \\ \text { N } & 32.634245 & 46.799915 & 29.437187 \\ \text { H } & 33.489030 & 46.317438 & 29.307262 \\ \mathrm{C} & 31.438656 & 46.060873 & 29.678940 \\ \mathrm{C} & 31.462855 & 44.636874 & 29.105950 \\ \mathrm{~S} & 32.168049 & 43.345309 & 30.220871 \\ \mathrm{C} & 33.663786 & 42.810649 & 29.309667 \\ \mathrm{C} & 34.895165 & 43.503993 & 29.638694 \\ \mathrm{C} & 36.244933 & 42.947753 & 29.589258 \\ \mathrm{C} & 36.844734 & 42.127320 & 30.561817 \\ \mathrm{C} & 36.369443 & 41.611848 & 31.797230 \\ \mathrm{C} & 37.238162 & 40.933599 & 32.630288 \\ \mathrm{C} & 38.622919 & 40.753873 & 32.335359\end{array}$




$\begin{array}{llll}\text { O } & 39.418224 & 40.122418 & 33.140673 \\ \text { C } & 39.073208 & 41.254247 & 31.080271 \\ \text { C } & 38.210741 & 41.907627 & 30.233932 \\ \text { C } & 38.521710 & 42.521368 & 28.890307 \\ \text { C } & 37.173159 & 43.093818 & 28.400196 \\ \text { H } & 37.259584 & 44.124241 & 28.062874 \\ \text { H } & 36.794676 & 42.515659 & 27.559512 \\ \text { H } & 38.906064 & 41.775255 & 28.206174 \\ \text { H } & 39.276257 & 43.297376 & 28.967590 \\ \text { H } & 40.106314 & 41.109272 & 30.812997 \\ \text { H } & 36.874326 & 40.527503 & 33.557433 \\ \text { H } & 35.335749 & 41.713433 & 32.075644 \\ \text { H } & 34.736723 & 44.417641 & 30.182002 \\ \text { O } & 33.566846 & 41.939640 & 28.481028 \\ \text { H } & 30.445189 & 44.325586 & 28.928271 \\ \text { H } & 31.985337 & 44.618850 & 28.159390 \\ \text { H } & 30.605775 & 46.559309 & 29.190835 \\ \text { C } & 31.027998 & 46.033120 & 31.153108 \\ \text { O } & 29.905822 & 45.829862 & 31.479964 \\ \text { H } & 32.673530 & 47.760275 & 29.648958 \\ \text { H } & 31.788529 & 46.226305 & 31.855861 \\ \text { H } & 45.434195 & 40.069501 & 36.574123 \\ \text { H } & 41.503438 & 35.107229 & 33.824005\end{array}$

$\begin{array}{lrll}\text { C7-C10-C11-C12-90 } & & \\ \text { C } & 44.697978 & 39.911772 & 35.813951 \\ \text { C } & 44.985908 & 39.195813 & 34.659216 \\ \text { H } & 45.922873 & 38.682591 & 34.540009 \\ \text { C } & 44.067696 & 39.134695 & 33.632229 \\ \text { H } & 44.299806 & 38.599498 & 32.731310 \\ \text { C } & 42.847663 & 39.796636 & 33.731030 \\ \text { O } & 42.014561 & 39.748927 & 32.695270 \\ \text { H } & 41.089293 & 39.966170 & 32.920084 \\ \text { C } & 42.550276 & 40.493938 & 34.896973 \\ \text { H } & 41.606372 & 40.998973 & 34.978018 \\ \text { C } & 43.474205 & 40.557682 & 35.920488 \\ \text { H } & 43.227630 & 41.128228 & 36.799056 \\ \text { C } & 41.493872 & 35.933581 & 34.532026 \\ \text { H } & 41.194755 & 35.565266 & 35.506683 \\ \text { H } & 42.509415 & 36.305116 & 34.621123 \\ \text { C } & 40.581538 & 37.086276 & 34.111006 \\ \text { H } & 40.726874 & 37.915652 & 34.796867 \\ \text { H } & 40.852605 & 37.491669 & 33.142233 \\ \text { C } & 39.094903 & 36.799330 & 34.093919\end{array}$




\begin{tabular}{|c|c|c|c|}
\hline 0 & 38.631874 & 35.722229 & 34.319407 \\
\hline 0 & 38.325113 & 37.816908 & 33.820541 \\
\hline $\mathrm{H}$ & 38.787825 & 38.667326 & 33.633376 \\
\hline $\mathrm{N}$ & 32.606108 & 46.759075 & 29.395516 \\
\hline $\mathrm{H}$ & 33.455886 & 46.266507 & 29.270370 \\
\hline C & 31.411851 & 46.031414 & 29.663300 \\
\hline C & 31.427412 & 44.603973 & 29.105037 \\
\hline$S$ & 32.242713 & 43.358348 & 30.192733 \\
\hline C & 33.674684 & 42.822553 & 29.186569 \\
\hline C & 34.953701 & 43.398705 & 29.563559 \\
\hline C & 36.267073 & 42.765741 & 29.490695 \\
\hline C & 36.865716 & 42.012371 & 30.515936 \\
\hline C & 36.371415 & 41.540808 & 31.761036 \\
\hline C & 37.231002 & 40.906427 & 32.636543 \\
\hline C & 38.623340 & 40.727702 & 32.376973 \\
\hline 0 & 39.403338 & 40.122765 & 33.216317 \\
\hline C & 39.095411 & 41.195090 & 31.116994 \\
\hline C & 38.241944 & 41.802681 & 30.228375 \\
\hline C & 38.564332 & 42.331052 & 28.852640 \\
\hline C & 37.256610 & 42.998582 & 28.366969 \\
\hline $\mathrm{H}$ & 37.393745 & 44.059710 & 28.164705 \\
\hline $\mathrm{H}$ & 36.908583 & 42.543512 & 27.443315 \\
\hline $\mathrm{H}$ & 38.843294 & 41.511946 & 28.199210 \\
\hline $\mathrm{H}$ & 39.398044 & 43.025309 & 28.860917 \\
\hline $\mathrm{H}$ & 40.136082 & 41.057740 & 30.876814 \\
\hline $\mathrm{H}$ & 36.850790 & 40.537091 & 33.572199 \\
\hline $\mathrm{H}$ & 35.330666 & 41.640797 & 32.013001 \\
\hline $\mathrm{H}$ & 34.856170 & 44.330717 & 30.089470 \\
\hline 0 & 33.505892 & 42.043807 & 28.281144 \\
\hline $\mathrm{H}$ & 30.408796 & 44.266564 & 29.004306 \\
\hline $\mathrm{H}$ & 31.893606 & 44.592244 & 28.127934 \\
\hline $\mathrm{H}$ & 30.572710 & 46.526677 & 29.181765 \\
\hline C & 31.020993 & 46.021529 & 31.141835 \\
\hline 0 & 29.901461 & 45.822317 & 31.476350 \\
\hline $\mathrm{H}$ & 32.656862 & 47.721512 & 29.595512 \\
\hline $\mathrm{H}$ & 31.789606 & 46.218305 & 31.836565 \\
\hline $\mathrm{H}$ & 45.465155 & 40.056616 & 36.588875 \\
\hline $\mathrm{H}$ & 41.491080 & 35.079354 & 33.819240 \\
\hline \multicolumn{4}{|c|}{ C7-C10-C11-C12-100 } \\
\hline C & 44.717960 & 39.930095 & 35.831233 \\
\hline C & 45.018984 & 39.249270 & 34.658283 \\
\hline $\mathrm{H}$ & 45.964983 & 38.754953 & 34.523867 \\
\hline C & 44.103892 & 39.200092 & 33.628229 \\
\hline
\end{tabular}




\begin{tabular}{|c|c|c|c|}
\hline $\mathrm{H}$ & 44.342541 & 38.688835 & 32.714986 \\
\hline C & 42.870415 & 39.834344 & 33.745548 \\
\hline 0 & 42.038164 & 39.795458 & 32.710736 \\
\hline $\mathrm{H}$ & 41.108404 & 40.000509 & 32.935887 \\
\hline$C$ & 42.562111 & 40.499597 & 34.927465 \\
\hline $\mathrm{H}$ & 41.609392 & 40.986021 & 35.021173 \\
\hline C & 43.483403 & 40.551885 & 35.953563 \\
\hline $\mathrm{H}$ & 43.227187 & 41.095396 & 36.846357 \\
\hline C & 41.514979 & 35.932743 & 34.511363 \\
\hline $\mathrm{H}$ & 41.213264 & 35.575799 & 35.489763 \\
\hline $\mathrm{H}$ & 42.532314 & 36.300760 & 34.595144 \\
\hline C & 40.609357 & 37.086573 & 34.078108 \\
\hline $\mathrm{H}$ & 40.762304 & 37.926908 & 34.748123 \\
\hline $\mathrm{H}$ & 40.879657 & 37.473797 & 33.101366 \\
\hline C & 39.121134 & 36.805877 & 34.065963 \\
\hline 0 & 38.653355 & 35.727158 & 34.273873 \\
\hline 0 & 38.354148 & 37.831142 & 33.810777 \\
\hline $\mathrm{H}$ & 38.821038 & 38.680100 & 33.635392 \\
\hline $\mathrm{N}$ & 32.634767 & 46.704867 & 29.338630 \\
\hline $\mathrm{H}$ & 33.487781 & 46.213296 & 29.232195 \\
\hline C & 31.439416 & 45.978736 & 29.610902 \\
\hline C & 31.447146 & 44.552670 & 29.049847 \\
\hline$S$ & 32.352570 & 43.325030 & 30.080790 \\
\hline C & 33.739186 & 42.810231 & 29.000926 \\
\hline C & 35.043411 & 43.279832 & 29.440451 \\
\hline$C$ & 36.328379 & 42.593921 & 29.341943 \\
\hline C & 36.933558 & 41.905476 & 30.411862 \\
\hline C & 36.416307 & 41.445494 & 31.651437 \\
\hline C & 37.267264 & 40.850721 & 32.561783 \\
\hline C & 38.673104 & 40.716315 & 32.347566 \\
\hline 0 & 39.441294 & 40.140883 & 33.217566 \\
\hline C & 39.166998 & 41.169137 & 31.091322 \\
\hline$C$ & 38.321803 & 41.737194 & 30.168660 \\
\hline C & 38.656055 & 42.222056 & 28.780942 \\
\hline C & 37.368857 & 42.919747 & 28.287454 \\
\hline $\mathrm{H}$ & 37.509620 & 43.996614 & 28.195038 \\
\hline $\mathrm{H}$ & 37.075102 & 42.554551 & 27.307771 \\
\hline $\mathrm{H}$ & 38.890664 & 41.371554 & 28.149551 \\
\hline $\mathrm{H}$ & 39.516873 & 42.881951 & 28.759833 \\
\hline $\mathrm{H}$ & 40.217821 & 41.060259 & 30.884149 \\
\hline $\mathrm{H}$ & 36.871484 & 40.492886 & 33.495344 \\
\hline $\mathrm{H}$ & 35.364092 & 41.503098 & 31.864872 \\
\hline $\mathrm{H}$ & 34.998257 & 44.232179 & 29.935190 \\
\hline 0 & 33.528681 & 42.128202 & 28.028944 \\
\hline
\end{tabular}




$\begin{array}{llll}\text { H } & 30.429436 & 44.200647 & 29.008335 \\ \text { H } & 31.856854 & 44.553143 & 28.048014 \\ \text { H } & 30.602022 & 46.470819 & 29.122210 \\ \text { C } & 31.057730 & 45.992879 & 31.092856 \\ \text { O } & 29.940256 & 45.810796 & 31.444188 \\ \text { H } & 32.680171 & 47.669927 & 29.530783 \\ \text { H } & 31.831887 & 46.200263 & 31.777304 \\ \text { H } & 45.480179 & 40.066966 & 36.611894 \\ \text { H } & 41.508625 & 35.071956 & 33.806670\end{array}$

$\begin{array}{lrll}\text { C7-C10-C11-C12-110 } & & \\ \mathrm{C} & 44.754288 & 39.937384 & 35.827781 \\ \mathrm{C} & 45.065859 & 39.271229 & 34.650514 \\ \mathrm{H} & 46.014387 & 38.780629 & 34.526573 \\ \mathrm{C} & 44.160439 & 39.226353 & 33.611332 \\ \mathrm{H} & 44.408204 & 38.724195 & 32.695125 \\ \mathrm{C} & 42.925048 & 39.854898 & 33.726181 \\ \mathrm{O} & 42.099218 & 39.822936 & 32.685669 \\ \mathrm{H} & 41.168198 & 40.015521 & 32.916923 \\ \mathrm{C} & 42.603101 & 40.506779 & 34.912190 \\ \mathrm{H} & 41.644510 & 40.982576 & 34.999862 \\ \mathrm{C} & 43.516718 & 40.553022 & 35.945775 \\ \mathrm{H} & 43.254333 & 41.086473 & 36.842856 \\ \mathrm{C} & 41.586051 & 35.958743 & 34.525062 \\ \mathrm{H} & 41.320106 & 35.601663 & 35.513688 \\ \mathrm{H} & 42.611842 & 36.310169 & 34.576415 \\ \mathrm{C} & 40.679237 & 37.124132 & 34.133940 \\ \mathrm{H} & 40.894850 & 37.966610 & 34.784290 \\ \mathrm{H} & 40.882901 & 37.495416 & 33.134986 \\ \mathrm{C} & 39.193498 & 36.860239 & 34.235159 \\ \mathrm{O} & 38.736551 & 35.797837 & 34.529769 \\ \mathrm{O} & 38.417972 & 37.879176 & 33.980603 \\ \mathrm{H} & 38.882416 & 38.710572 & 33.737107 \\ \mathrm{~N} & 32.644024 & 46.700871 & 29.285825 \\ \mathrm{H} & 33.494399 & 46.198265 & 29.210879 \\ \mathrm{C} & 31.445528 & 45.985170 & 29.561956 \\ \mathrm{C} & 31.453868 & 44.551342 & 29.022467 \\ \mathrm{~S} & 32.429721 & 43.370543 & 30.034986 \\ \mathrm{C} & 33.773291 & 42.811195 & 28.932880 \\ \mathrm{C} & 35.090742 & 43.159474 & 29.435015 \\ \mathrm{C} & 36.351576 & 42.436313 & 29.304923 \\ \mathrm{C} & 36.981493 & 41.802349 & 30.396109 \\ \mathrm{C} & 36.475078 & 41.368188 & 31.649246 \\ \mathrm{C} & 37.338261 & 40.817400 & 32.574157\end{array}$




\begin{tabular}{|c|c|c|c|}
\hline C & 38.745518 & 40.698794 & 32.355321 \\
\hline 0 & 39.519766 & 40.147346 & 33.233222 \\
\hline C & 39.230918 & 41.136436 & 31.091405 \\
\hline C & 38.371194 & 41.656966 & 30.154026 \\
\hline C & 38.687512 & 42.114047 & 28.753908 \\
\hline C & 37.400236 & 42.821125 & 28.276441 \\
\hline $\mathrm{H}$ & 37.531179 & 43.903240 & 28.243887 \\
\hline $\mathrm{H}$ & 37.120720 & 42.504887 & 27.276387 \\
\hline $\mathrm{H}$ & 38.888085 & 41.247683 & 28.131076 \\
\hline $\mathrm{H}$ & 39.559294 & 42.758038 & 28.703310 \\
\hline $\mathrm{H}$ & 40.282271 & 41.038402 & 30.882338 \\
\hline $\mathrm{H}$ & 36.957033 & 40.475505 & 33.519612 \\
\hline $\mathrm{H}$ & 35.424199 & 41.425912 & 31.867011 \\
\hline $\mathrm{H}$ & 35.111346 & 44.137621 & 29.878306 \\
\hline 0 & 33.532533 & 42.190137 & 27.928978 \\
\hline $\mathrm{H}$ & 30.443409 & 44.177380 & 29.036734 \\
\hline $\mathrm{H}$ & 31.816666 & 44.545418 & 28.003483 \\
\hline $\mathrm{H}$ & 30.608041 & 46.458388 & 29.055206 \\
\hline C & 31.055414 & 46.026050 & 31.040648 \\
\hline 0 & 29.929484 & 45.878314 & 31.387351 \\
\hline $\mathrm{H}$ & 32.698698 & 47.665961 & 29.479482 \\
\hline $\mathrm{H}$ & 31.833086 & 46.209390 & 31.730844 \\
\hline $\mathrm{H}$ & 45.510916 & 40.066687 & 36.614437 \\
\hline $\mathrm{H}$ & 41.540714 & 35.099995 & 33.820228 \\
\hline \multicolumn{4}{|c|}{$\mathrm{C} 7-\mathrm{C} 10-\mathrm{C} 11-\mathrm{C} 12-120^{\circ}$} \\
\hline C & 44.780621 & 39.951694 & 35.813936 \\
\hline C & 45.091932 & 39.289753 & 34.633485 \\
\hline $\mathrm{H}$ & 46.031217 & 38.783279 & 34.513688 \\
\hline C & 44.192261 & 39.258198 & 33.589686 \\
\hline $\mathrm{H}$ & 44.442178 & 38.755771 & 32.674304 \\
\hline C & 42.963364 & 39.900961 & 33.698056 \\
\hline 0 & 42.140219 & 39.872952 & 32.655769 \\
\hline $\mathrm{H}$ & 41.206553 & 40.052193 & 32.889344 \\
\hline C & 42.645720 & 40.557589 & 34.882865 \\
\hline $\mathrm{H}$ & 41.695586 & 41.049997 & 34.968421 \\
\hline C & 43.552429 & 40.587471 & 35.923665 \\
\hline $\mathrm{H}$ & 43.289472 & 41.118968 & 36.822248 \\
\hline C & 41.703792 & 35.996706 & 34.584909 \\
\hline $\mathrm{H}$ & 41.495658 & 35.665525 & 35.595080 \\
\hline $\mathrm{H}$ & 42.736128 & 36.327725 & 34.554428 \\
\hline C & 40.797794 & 37.174917 & 34.234989 \\
\hline $\mathrm{H}$ & 41.097824 & 38.032968 & 34.828387 \\
\hline $\mathrm{H}$ & 40.904348 & 37.499606 & 33.205492 \\
\hline
\end{tabular}




\begin{tabular}{|c|c|c|c|}
\hline C & 39.329621 & 36.962276 & 34.522510 \\
\hline 0 & 38.907135 & 35.961900 & 35.018135 \\
\hline 0 & 38.535275 & 37.949946 & 34.210071 \\
\hline $\mathrm{H}$ & 38.980406 & 38.754814 & 33.859857 \\
\hline$N$ & 32.541491 & 46.780045 & 29.383458 \\
\hline $\mathrm{H}$ & 33.384343 & 46.274968 & 29.258922 \\
\hline C & 31.344604 & 46.058583 & 29.657696 \\
\hline C & 31.354448 & 44.629257 & 29.099354 \\
\hline$S$ & 32.330762 & 43.439603 & 30.107777 \\
\hline C & 33.655262 & 42.824065 & 29.011708 \\
\hline C & 34.972573 & 43.061963 & 29.568653 \\
\hline C & 36.211789 & 42.310716 & 29.374723 \\
\hline C & 36.901026 & 41.705561 & 30.448946 \\
\hline C & 36.442931 & 41.261300 & 31.716413 \\
\hline C & 37.348175 & 40.748861 & 32.621852 \\
\hline C & 38.753601 & 40.670338 & 32.364148 \\
\hline 0 & 39.565643 & 40.156811 & 33.228097 \\
\hline C & 39.189048 & 41.117274 & 31.085802 \\
\hline C & 38.286704 & 41.602766 & 30.169044 \\
\hline C & 38.546709 & 42.068593 & 28.760796 \\
\hline C & 37.232348 & 42.758647 & 28.339939 \\
\hline $\mathrm{H}$ & 37.334781 & 43.844739 & 28.349142 \\
\hline $\mathrm{H}$ & 36.937152 & 42.476017 & 27.334921 \\
\hline $\mathrm{H}$ & 38.730020 & 41.207414 & 28.125458 \\
\hline $\mathrm{H}$ & 39.408081 & 42.723176 & 28.679547 \\
\hline $\mathrm{H}$ & 40.237705 & 41.057476 & 30.849843 \\
\hline $\mathrm{H}$ & 37.002289 & 40.401811 & 33.578964 \\
\hline $\mathrm{H}$ & 35.396919 & 41.292043 & 31.960293 \\
\hline $\mathrm{H}$ & 35.060304 & 44.055551 & 29.968520 \\
\hline 0 & 33.404757 & 42.239436 & 27.988061 \\
\hline $\mathrm{H}$ & 30.342545 & 44.255005 & 29.105248 \\
\hline $\mathrm{H}$ & 31.724943 & 44.629461 & 28.082991 \\
\hline $\mathrm{H}$ & 30.500014 & 46.544774 & 29.175803 \\
\hline C & 30.972960 & 46.068967 & 31.141946 \\
\hline 0 & 29.853366 & 45.909703 & 31.499488 \\
\hline $\mathrm{H}$ & 32.607655 & 47.740239 & 29.588036 \\
\hline $\mathrm{H}$ & 31.756983 & 46.253601 & 31.821457 \\
\hline $\mathrm{H}$ & 45.536800 & 40.073418 & 36.602698 \\
\hline $\mathrm{H}$ & 41.588072 & 35.129105 & 33.897268 \\
\hline \multicolumn{4}{|c|}{ C7-C10-C11-C12-130 } \\
\hline C & 44.801017 & 39.950991 & 35.816316 \\
\hline C & 45.120374 & 39.288325 & 34.638341 \\
\hline $\mathrm{H}$ & 46.059499 & 38.779497 & 34.528168 \\
\hline
\end{tabular}




\begin{tabular}{|c|c|c|c|}
\hline C & 44.227761 & 39.256937 & 33.589193 \\
\hline $\mathrm{H}$ & 44.481185 & 38.754183 & 32.675100 \\
\hline C & 42.996838 & 39.897540 & 33.691356 \\
\hline 0 & 42.180303 & 39.862676 & 32.643743 \\
\hline $\mathrm{H}$ & 41.246313 & 40.050832 & 32.867544 \\
\hline$C$ & 42.669830 & 40.552110 & 34.874396 \\
\hline $\mathrm{H}$ & 41.715919 & 41.038450 & 34.953579 \\
\hline C & 43.571540 & 40.584418 & 35.920174 \\
\hline $\mathrm{H}$ & 43.303394 & 41.117080 & 36.816366 \\
\hline$C$ & 41.706093 & 36.011988 & 34.564949 \\
\hline $\mathrm{H}$ & 41.502056 & 35.670496 & 35.573021 \\
\hline $\mathrm{H}$ & 42.740536 & 36.335758 & 34.535117 \\
\hline C & 40.803959 & 37.197436 & 34.235355 \\
\hline $\mathrm{H}$ & 41.111278 & 38.048350 & 34.834922 \\
\hline $\mathrm{H}$ & 40.903051 & 37.535428 & 33.209472 \\
\hline C & 39.337534 & 36.984760 & 34.528809 \\
\hline 0 & 38.914350 & 35.986854 & 35.028109 \\
\hline 0 & 38.544453 & 37.974080 & 34.216740 \\
\hline $\mathrm{H}$ & 38.996754 & 38.770113 & 33.860873 \\
\hline $\mathrm{N}$ & 32.476063 & 46.752076 & 29.351923 \\
\hline $\mathrm{H}$ & 33.317444 & 46.239940 & 29.242494 \\
\hline C & 31.284741 & 46.032259 & 29.650584 \\
\hline C & 31.304808 & 44.594539 & 29.109316 \\
\hline$S$ & 32.366206 & 43.463156 & 30.088050 \\
\hline C & 33.618074 & 42.791890 & 28.954118 \\
\hline C & 34.937741 & 42.888687 & 29.554796 \\
\hline C & 36.156204 & 42.106349 & 29.334241 \\
\hline C & 36.881090 & 41.554117 & 30.413747 \\
\hline C & 36.447214 & 41.141029 & 31.700067 \\
\hline C & 37.374424 & 40.687231 & 32.612164 \\
\hline C & 38.779653 & 40.635559 & 32.337647 \\
\hline 0 & 39.609739 & 40.160832 & 33.205289 \\
\hline C & 39.191560 & 41.066251 & 31.046877 \\
\hline C & 38.265283 & 41.488684 & 30.121834 \\
\hline C & 38.493258 & 41.929742 & 28.700587 \\
\hline C & 37.156196 & 42.579897 & 28.288753 \\
\hline $\mathrm{H}$ & 37.227527 & 43.669080 & 28.299015 \\
\hline $\mathrm{H}$ & 36.856615 & 42.290377 & 27.286693 \\
\hline $\mathrm{H}$ & 38.685735 & 41.060809 & 28.078144 \\
\hline $\mathrm{H}$ & 39.337052 & 42.603603 & 28.594571 \\
\hline $\mathrm{H}$ & 40.239232 & 41.026332 & 30.802489 \\
\hline $\mathrm{H}$ & 37.049386 & 40.361068 & 33.583554 \\
\hline $\mathrm{H}$ & 35.403651 & 41.159980 & 31.954011 \\
\hline $\mathrm{H}$ & 35.104989 & 43.899841 & 29.886380 \\
\hline
\end{tabular}




$\begin{array}{llll}\mathrm{O} & 33.332396 & 42.296717 & 27.894759 \\ \mathrm{H} & 30.306981 & 44.188310 & 29.162674 \\ \mathrm{H} & 31.633615 & 44.599135 & 28.078640 \\ \mathrm{H} & 30.432832 & 46.499699 & 29.163026 \\ \mathrm{C} & 30.915943 & 46.063285 & 31.136203 \\ \mathrm{O} & 29.796637 & 45.899892 & 31.496250 \\ \mathrm{H} & 32.552941 & 47.709919 & 29.570907 \\ \mathrm{H} & 31.700786 & 46.254955 & 31.815121 \\ \mathrm{H} & 45.550638 & 40.066290 & 36.612059 \\ \mathrm{H} & 41.583284 & 35.151430 & 33.870276\end{array}$

$\begin{array}{lrll}\text { C7-C10-C11-C12-140 } & & \\ \mathrm{C} & 44.810548 & 39.939007 & 35.792556 \\ \mathrm{C} & 45.142627 & 39.270147 & 34.621912 \\ \mathrm{H} & 46.082315 & 38.759833 & 34.524820 \\ \mathrm{C} & 44.263436 & 39.235485 & 33.561813 \\ \mathrm{H} & 44.526640 & 38.728433 & 32.652787 \\ \mathrm{C} & 43.032982 & 39.878443 & 33.645694 \\ \mathrm{O} & 42.229162 & 39.840913 & 32.587384 \\ \mathrm{H} & 41.297855 & 40.039800 & 32.803360 \\ \mathrm{C} & 42.691594 & 40.538009 & 34.821285 \\ \mathrm{H} & 41.737561 & 41.026422 & 34.886499 \\ \mathrm{C} & 43.580638 & 40.573932 & 35.878114 \\ \mathrm{H} & 43.302364 & 41.111232 & 36.768368 \\ \mathrm{C} & 41.728065 & 35.995844 & 34.581833 \\ \mathrm{H} & 41.520167 & 35.657567 & 35.589945 \\ \mathrm{H} & 42.765463 & 36.309552 & 34.550805 \\ \mathrm{C} & 40.838598 & 37.189833 & 34.250474 \\ \mathrm{H} & 41.155038 & 38.038947 & 34.848218 \\ \mathrm{H} & 40.941418 & 37.524262 & 33.223917 \\ \mathrm{C} & 39.370897 & 36.991532 & 34.546076 \\ \mathrm{O} & 38.938623 & 36.005746 & 35.059453 \\ \mathrm{O} & 38.584041 & 37.982346 & 34.217444 \\ \mathrm{H} & 39.041747 & 38.765233 & 33.850126 \\ \mathrm{~N} & 32.496792 & 46.811072 & 29.306284 \\ \mathrm{H} & 33.335001 & 46.297498 & 29.179969 \\ \mathrm{C} & 31.301205 & 46.091132 & 29.601384 \\ \mathrm{C} & 31.310924 & 44.658808 & 29.046892 \\ \mathrm{~S} & 32.342827 & 43.503134 & 30.025024 \\ \mathrm{C} & 33.587361 & 42.791539 & 28.907857 \\ \mathrm{C} & 34.873277 & 42.788313 & 29.582464 \\ \mathrm{C} & 36.093605 & 42.002965 & 29.345196 \\ \mathrm{C} & 36.858550 & 41.498506 & 30.415240 \\ \mathrm{C} & 36.459940 & 41.109784 & 31.721776\end{array}$




\begin{tabular}{|c|c|c|c|}
\hline C & 37.410488 & 40.678438 & 32.617612 \\
\hline C & 38.810638 & 40.627532 & 32.306234 \\
\hline 0 & 39.657324 & 40.167072 & 33.156867 \\
\hline C & 39.188146 & 41.044665 & 30.998364 \\
\hline C & 38.237156 & 41.441783 & 30.089726 \\
\hline C & 38.422144 & 41.867138 & 28.657026 \\
\hline C & 37.058740 & 42.477146 & 28.267468 \\
\hline $\mathrm{H}$ & 37.101714 & 43.567921 & 28.250173 \\
\hline $\mathrm{H}$ & 36.738592 & 42.155430 & 27.281558 \\
\hline $\mathrm{H}$ & 38.621721 & 40.996664 & 28.039252 \\
\hline $\mathrm{H}$ & 39.245504 & 42.561440 & 28.525252 \\
\hline $\mathrm{H}$ & 40.230761 & 41.011881 & 30.731704 \\
\hline $\mathrm{H}$ & 37.113165 & 40.368727 & 33.603199 \\
\hline $\mathrm{H}$ & 35.423274 & 41.133734 & 32.001905 \\
\hline $\mathrm{H}$ & 35.085847 & 43.807132 & 29.872710 \\
\hline 0 & 33.313306 & 42.342065 & 27.825342 \\
\hline $\mathrm{H}$ & 30.307370 & 44.265822 & 29.095132 \\
\hline $\mathrm{H}$ & 31.651779 & 44.665880 & 28.020541 \\
\hline $\mathrm{H}$ & 30.453069 & 46.564779 & 29.113546 \\
\hline C & 30.932898 & 46.107826 & 31.089234 \\
\hline 0 & 29.810736 & 45.973702 & 31.448719 \\
\hline $\mathrm{H}$ & 32.580087 & 47.764180 & 29.539958 \\
\hline $\mathrm{H}$ & 31.722291 & 46.264366 & 31.771471 \\
\hline $\mathrm{H}$ & 45.551138 & 40.059381 & 36.595442 \\
\hline $\mathrm{H}$ & 41.596444 & 35.135818 & 33.887585 \\
\hline \multicolumn{4}{|c|}{ C7-C10-C11-C12-150 } \\
\hline C & 44.832298 & 39.801987 & 35.749178 \\
\hline C & 45.220918 & 39.063312 & 34.640298 \\
\hline $\mathrm{H}$ & 46.167605 & 38.560760 & 34.618119 \\
\hline C & 44.399654 & 38.961089 & 33.538650 \\
\hline $\mathrm{H}$ & 44.708874 & 38.400655 & 32.677496 \\
\hline C & 43.172682 & 39.606934 & 33.526809 \\
\hline 0 & 42.423307 & 39.505602 & 32.423232 \\
\hline $\mathrm{H}$ & 41.508819 & 39.740134 & 32.578360 \\
\hline C & 42.767735 & 40.334552 & 34.637740 \\
\hline $\mathrm{H}$ & 41.812512 & 40.825677 & 34.626876 \\
\hline C & 43.599308 & 40.435721 & 35.736616 \\
\hline $\mathrm{H}$ & 43.274549 & 41.023642 & 36.577611 \\
\hline C & 41.743359 & 35.908734 & 34.608360 \\
\hline $\mathrm{H}$ & 41.565043 & 35.534271 & 35.608256 \\
\hline $\mathrm{H}$ & 42.771309 & 36.249610 & 34.572774 \\
\hline C & 40.809717 & 37.080218 & 34.325254 \\
\hline $\mathrm{H}$ & 41.128322 & 37.938431 & 34.910400 \\
\hline
\end{tabular}




\begin{tabular}{|c|c|c|c|}
\hline $\mathrm{H}$ & 40.852799 & 37.416810 & 33.295800 \\
\hline C & 39.366697 & 36.843798 & 34.694971 \\
\hline 0 & 38.986142 & 35.900937 & 35.307555 \\
\hline 0 & 38.523898 & 37.781249 & 34.313255 \\
\hline $\mathrm{H}$ & 38.960304 & 38.508287 & 33.865232 \\
\hline$N$ & 32.494491 & 46.820045 & 29.274241 \\
\hline $\mathrm{H}$ & 33.303252 & 46.283270 & 29.073911 \\
\hline C & 31.261141 & 46.151818 & 29.513595 \\
\hline C & 31.214734 & 44.732193 & 28.933833 \\
\hline$S$ & 32.066336 & 43.433228 & 29.889832 \\
\hline C & 33.696086 & 43.227630 & 29.052139 \\
\hline C & 34.716906 & 42.967199 & 29.998852 \\
\hline C & 36.067070 & 42.641775 & 29.760082 \\
\hline C & 36.841009 & 41.808147 & 30.671278 \\
\hline C & 36.489892 & 41.286017 & 31.939507 \\
\hline C & 37.452490 & 40.644025 & 32.697252 \\
\hline C & 38.807770 & 40.509829 & 32.242889 \\
\hline 0 & 39.681872 & 39.928233 & 32.938196 \\
\hline C & 39.124200 & 41.015219 & 30.928719 \\
\hline C & 38.148192 & 41.628979 & 30.171576 \\
\hline C & 38.268607 & 42.263843 & 28.806729 \\
\hline C & 36.831073 & 42.721229 & 28.459910 \\
\hline $\mathrm{H}$ & 36.815256 & 43.712802 & 28.024174 \\
\hline $\mathrm{H}$ & 36.389637 & 42.056527 & 27.721722 \\
\hline $\mathrm{H}$ & 38.641565 & 41.555227 & 28.077928 \\
\hline $\mathrm{H}$ & 38.959110 & 43.102999 & 28.829978 \\
\hline $\mathrm{H}$ & 40.131127 & 40.887882 & 30.571982 \\
\hline $\mathrm{H}$ & 37.207937 & 40.242167 & 33.663262 \\
\hline $\mathrm{H}$ & 35.487109 & 41.372268 & 32.307662 \\
\hline $\mathrm{H}$ & 34.375838 & 42.845935 & 31.008082 \\
\hline 0 & 33.756099 & 43.303815 & 27.828695 \\
\hline $\mathrm{H}$ & 30.180782 & 44.426720 & 28.906797 \\
\hline $\mathrm{H}$ & 31.601990 & 44.738439 & 27.923826 \\
\hline $\mathrm{H}$ & 30.441825 & 46.681970 & 29.033369 \\
\hline C & 30.854204 & 46.137441 & 30.989120 \\
\hline 0 & 29.719457 & 46.007152 & 31.329102 \\
\hline $\mathrm{H}$ & 32.601524 & 47.775418 & 29.480199 \\
\hline $\mathrm{H}$ & 31.630187 & 46.272997 & 31.689954 \\
\hline $\mathrm{H}$ & 45.533320 & 39.973459 & 36.578873 \\
\hline $\mathrm{H}$ & 41.621535 & 35.067003 & 33.891955 \\
\hline \multicolumn{4}{|c|}{ C7-C10-C11-C12 -160 } \\
\hline C & 44.836682 & 39.785588 & 35.769277 \\
\hline C & 45.225361 & 39.044082 & 34.662601 \\
\hline
\end{tabular}




\begin{tabular}{|c|c|c|c|}
\hline $\mathrm{H}$ & 46.170447 & 38.537134 & 34.640893 \\
\hline C & 44.407239 & 38.954798 & 33.557386 \\
\hline $\mathrm{H}$ & 44.717524 & 38.398458 & 32.694064 \\
\hline C & 43.187130 & 39.613242 & 33.541587 \\
\hline 0 & 42.437980 & 39.527547 & 32.434639 \\
\hline $\mathrm{H}$ & 41.529859 & 39.778156 & 32.598975 \\
\hline C & 42.784048 & 40.340868 & 34.652912 \\
\hline $\mathrm{H}$ & 41.834741 & 40.842844 & 34.640761 \\
\hline C & 43.609300 & 40.430597 & 35.756170 \\
\hline $\mathrm{H}$ & 43.284715 & 41.020582 & 36.595952 \\
\hline C & 41.785084 & 35.923393 & 34.610616 \\
\hline $\mathrm{H}$ & 41.616863 & 35.548852 & 35.612851 \\
\hline $\mathrm{H}$ & 42.813496 & 36.260321 & 34.558980 \\
\hline C & 40.853783 & 37.098273 & 34.343273 \\
\hline $\mathrm{H}$ & 41.169012 & 37.947011 & 34.944620 \\
\hline $\mathrm{H}$ & 40.903114 & 37.446893 & 33.318243 \\
\hline C & 39.408636 & 36.846012 & 34.695793 \\
\hline 0 & 39.025561 & 35.884054 & 35.276654 \\
\hline 0 & 38.564483 & 37.789643 & 34.329886 \\
\hline $\mathrm{H}$ & 39.003309 & 38.531359 & 33.912872 \\
\hline $\mathrm{N}$ & 32.461754 & 46.831086 & 29.265989 \\
\hline $\mathrm{H}$ & 33.253468 & 46.298503 & 28.997399 \\
\hline C & 31.229335 & 46.166248 & 29.511534 \\
\hline C & 31.195283 & 44.751487 & 28.923564 \\
\hline$S$ & 32.046656 & 43.454053 & 29.882452 \\
\hline C & 33.725470 & 43.342418 & 29.119887 \\
\hline C & 34.685042 & 42.910452 & 30.066274 \\
\hline C & 36.039083 & 42.635829 & 29.835115 \\
\hline C & 36.836151 & 41.807003 & 30.741438 \\
\hline C & 36.483893 & 41.235866 & 31.985321 \\
\hline C & 37.457330 & 40.610220 & 32.740357 \\
\hline C & 38.826594 & 40.551423 & 32.309430 \\
\hline 0 & 39.709140 & 39.992262 & 33.010766 \\
\hline C & 39.140272 & 41.094343 & 31.011203 \\
\hline C & 38.152796 & 41.694760 & 30.255471 \\
\hline C & 38.268273 & 42.366636 & 28.907333 \\
\hline C & 36.817499 & 42.767202 & 28.544983 \\
\hline $\mathrm{H}$ & 36.761732 & 43.758946 & 28.116954 \\
\hline $\mathrm{H}$ & 36.411459 & 42.089058 & 27.798203 \\
\hline $\mathrm{H}$ & 38.692306 & 41.694208 & 28.171434 \\
\hline $\mathrm{H}$ & 38.917951 & 43.236016 & 28.961886 \\
\hline $\mathrm{H}$ & 40.156923 & 41.015016 & 30.668714 \\
\hline $\mathrm{H}$ & 37.216755 & 40.176061 & 33.692748 \\
\hline $\mathrm{H}$ & 35.472681 & 41.274169 & 32.337841 \\
\hline
\end{tabular}




\begin{tabular}{|c|c|c|c|}
\hline $\mathrm{H}$ & 34.302908 & 42.677422 & 31.041233 \\
\hline 0 & 33.866796 & 43.601561 & 27.927612 \\
\hline $\mathrm{H}$ & 30.165193 & 44.433969 & 28.880557 \\
\hline $\mathrm{H}$ & 31.597696 & 44.761370 & 27.919505 \\
\hline $\mathrm{H}$ & 30.402828 & 46.699018 & 29.045385 \\
\hline C & 30.842631 & 46.139889 & 30.990637 \\
\hline 0 & 29.718222 & 45.989858 & 31.337554 \\
\hline $\mathrm{H}$ & 32.576407 & 47.785031 & 29.481693 \\
\hline $\mathrm{H}$ & 31.625236 & 46.279131 & 31.684887 \\
\hline $\mathrm{H}$ & 45.538054 & 39.954547 & 36.598833 \\
\hline $\mathrm{H}$ & 41.646391 & 35.084595 & 33.894160 \\
\hline \multicolumn{4}{|c|}{ C7-C10-C11-C12 - $170^{\circ}$} \\
\hline C & 44.841118 & 39.795319 & 35.776917 \\
\hline C & 45.230836 & 39.057540 & 34.668231 \\
\hline $\mathrm{H}$ & 46.178994 & 38.556808 & 34.644286 \\
\hline C & 44.413584 & 38.969130 & 33.561981 \\
\hline $\mathrm{H}$ & 44.725840 & 38.416656 & 32.696668 \\
\hline C & 43.192210 & 39.624994 & 33.547892 \\
\hline 0 & 42.443755 & 39.542464 & 32.439824 \\
\hline $\mathrm{H}$ & 41.536022 & 39.792822 & 32.605596 \\
\hline C & 42.786687 & 40.347236 & 34.661471 \\
\hline $\mathrm{H}$ & 41.836063 & 40.846837 & 34.651155 \\
\hline C & 43.611416 & 40.435211 & 35.765537 \\
\hline $\mathrm{H}$ & 43.285318 & 41.022502 & 36.606474 \\
\hline C & 41.800391 & 35.925120 & 34.621174 \\
\hline $\mathrm{H}$ & 41.638195 & 35.549979 & 35.624261 \\
\hline $\mathrm{H}$ & 42.828147 & 36.262444 & 34.562155 \\
\hline C & 40.867874 & 37.100013 & 34.358933 \\
\hline $\mathrm{H}$ & 41.183581 & 37.947355 & 34.960447 \\
\hline $\mathrm{H}$ & 40.917539 & 37.450002 & 33.334238 \\
\hline C & 39.422331 & 36.846495 & 34.709392 \\
\hline 0 & 39.037775 & 35.880961 & 35.282601 \\
\hline 0 & 38.579230 & 37.793499 & 34.349516 \\
\hline $\mathrm{H}$ & 39.017396 & 38.535485 & 33.932899 \\
\hline$N$ & 32.415299 & 46.814658 & 29.283730 \\
\hline $\mathrm{H}$ & 33.192378 & 46.282959 & 28.971878 \\
\hline C & 31.183956 & 46.156220 & 29.538825 \\
\hline C & 31.156768 & 44.743249 & 28.950279 \\
\hline$S$ & 32.032340 & 43.454559 & 29.892555 \\
\hline C & 33.736064 & 43.430800 & 29.174254 \\
\hline C & 34.656016 & 42.852556 & 30.079751 \\
\hline C & 36.008350 & 42.584414 & 29.841590 \\
\hline C & 36.825020 & 41.792701 & 30.761110 \\
\hline
\end{tabular}




$\begin{array}{llll}\text { C } & 36.482374 & 41.229397 & 32.011940 \\ \text { C } & 37.462114 & 40.614762 & 32.767118 \\ \text { C } & 38.830309 & 40.563747 & 32.331682 \\ \text { O } & 39.716796 & 40.004334 & 33.027238 \\ \text { C } & 39.139348 & 41.112963 & 31.035114 \\ \text { C } & 38.145479 & 41.703707 & 30.280261 \\ \text { C } & 38.252467 & 42.373824 & 28.930180 \\ \text { C } & 36.796323 & 42.750747 & 28.562285 \\ \text { H } & 36.722996 & 43.745165 & 28.144168 \\ \text { H } & 36.409588 & 42.073160 & 27.803349 \\ \text { H } & 38.688946 & 41.705560 & 28.197566 \\ \text { H } & 38.887702 & 43.253670 & 28.984652 \\ \text { H } & 40.157160 & 41.046298 & 30.693113 \\ \text { H } & 37.227774 & 40.182313 & 33.721911 \\ \text { H } & 35.471433 & 41.258824 & 32.365476 \\ \text { H } & 34.255902 & 42.551292 & 31.028164 \\ \text { O } & 33.925210 & 43.859915 & 28.038583 \\ \text { H } & 30.131111 & 44.410764 & 28.916857 \\ \text { H } & 31.551204 & 44.764034 & 27.943223 \\ \text { H } & 30.351465 & 46.684543 & 29.077642 \\ \text { C } & 30.813756 & 46.130891 & 31.020969 \\ \text { O } & 29.693764 & 45.972433 & 31.379928 \\ \text { H } & 32.541539 & 47.76688 & 29.504769 \\ \text { H } & 31.602787 & 46.280414 & 31.705809 \\ \text { H } & 45.541378 & 39.962894 & 36.607668 \\ \text { H } & 41.657095 & 35.087296 & 33.904666\end{array}$

The QM(CASSCF)/MM calculated minimum-energy isomerization path connecting both S0-cisPMIN and S0-cisT-MIN of the single-bond-rotation locked PYP chromophore in the wild-type protein.

$\begin{array}{llll}\text { Point 1 } & & & \\ \text { C } & 44.778020 & 39.881950 & 35.773130 \\ \text { C } & 45.118549 & 39.187379 & 34.619710 \\ \text { H } & 46.062488 & 38.685600 & 34.530456 \\ \text { C } & 44.249317 & 39.134883 & 33.550974 \\ \text { H } & 44.524423 & 38.615896 & 32.653180 \\ \text { C } & 43.023410 & 39.786859 & 33.611612 \\ \text { O } & 42.236354 & 39.745590 & 32.538008 \\ \text { H } & 41.322142 & 40.008127 & 32.715534 \\ \text { C } & 42.668258 & 40.463823 & 34.772361 \\ \text { H } & 41.716791 & 40.959017 & 34.822839 \\ \text { C } & 43.547159 & 40.517173 & 35.836760\end{array}$




\begin{tabular}{|c|c|c|c|}
\hline $\mathrm{H}$ & 43.262110 & 41.077946 & 36.709968 \\
\hline C & 41.651126 & 35.964123 & 34.568653 \\
\hline $\mathrm{H}$ & 41.399449 & 35.600312 & 35.557309 \\
\hline $\mathrm{H}$ & 42.673579 & 36.320625 & 34.599214 \\
\hline C & 40.740373 & 37.132604 & 34.188399 \\
\hline $\mathrm{H}$ & 40.945678 & 37.974703 & 34.845302 \\
\hline $\mathrm{H}$ & 40.953458 & 37.514267 & 33.194462 \\
\hline C & 39.256306 & 36.871312 & 34.270277 \\
\hline 0 & 38.784237 & 35.833353 & 34.612374 \\
\hline 0 & 38.486161 & 37.882313 & 33.945883 \\
\hline $\mathrm{H}$ & 38.961718 & 38.679819 & 33.668672 \\
\hline$N$ & 32.633698 & 46.886657 & 29.536896 \\
\hline $\mathrm{H}$ & 33.487013 & 46.373933 & 29.542728 \\
\hline C & 31.427542 & 46.145703 & 29.730086 \\
\hline C & 31.558479 & 44.721367 & 29.140305 \\
\hline$S$ & 31.793444 & 43.375804 & 30.347794 \\
\hline C & 33.516020 & 42.787460 & 30.537399 \\
\hline C & 34.543849 & 42.998734 & 29.550828 \\
\hline C & 35.864012 & 42.597914 & 29.658635 \\
\hline C & 36.652805 & 41.917092 & 30.630455 \\
\hline C & 36.332648 & 41.215279 & 31.827574 \\
\hline C & 37.321597 & 40.666149 & 32.597836 \\
\hline C & 38.718234 & 40.757573 & 32.271335 \\
\hline 0 & 39.593993 & 40.207747 & 32.999384 \\
\hline C & 39.031159 & 41.454055 & 31.061680 \\
\hline C & 38.032414 & 42.003005 & 30.296876 \\
\hline C & 38.215382 & 42.794083 & 29.028968 \\
\hline C & 36.789000 & 42.943387 & 28.492887 \\
\hline $\mathrm{H}$ & 36.590018 & 43.935241 & 28.100049 \\
\hline $\mathrm{H}$ & 36.607985 & 42.229664 & 27.693078 \\
\hline $\mathrm{H}$ & 38.870507 & 42.294734 & 28.326141 \\
\hline $\mathrm{H}$ & 38.655478 & 43.764071 & 29.238074 \\
\hline $\mathrm{H}$ & 40.066275 & 41.562875 & 30.785140 \\
\hline $\mathrm{H}$ & 37.055206 & 40.136374 & 33.493859 \\
\hline $\mathrm{H}$ & 35.318752 & 41.079771 & 32.123910 \\
\hline $\mathrm{H}$ & 34.263569 & 43.543850 & 28.669224 \\
\hline 0 & 33.598084 & 42.169363 & 31.552239 \\
\hline $\mathrm{H}$ & 30.631732 & 44.457508 & 28.652015 \\
\hline $\mathrm{H}$ & 32.329768 & 44.713693 & 28.384291 \\
\hline $\mathrm{H}$ & 30.617025 & 46.637833 & 29.206637 \\
\hline C & 30.996854 & 46.139226 & 31.199909 \\
\hline 0 & 29.863261 & 46.052183 & 31.547692 \\
\hline $\mathrm{H}$ & 32.674645 & 47.857440 & 29.665476 \\
\hline $\mathrm{H}$ & 31.783879 & 46.262575 & 31.886747 \\
\hline
\end{tabular}




$\begin{array}{llll}\mathrm{H} & 45.510819 & 40.020000 & 36.582294 \\ \mathrm{H} & 41.593699 & 35.110336 & 33.856862\end{array}$

Point 2

\begin{tabular}{|c|c|c|c|}
\hline C & 44.760789 & 39.870730 & 35.757607 \\
\hline C & 45.113316 & 39.189100 & 34.598997 \\
\hline $\mathrm{H}$ & 46.058834 & 38.690068 & 34.510568 \\
\hline C & 44.250383 & 39.140447 & 33.525333 \\
\hline $\mathrm{H}$ & 44.534284 & 38.632289 & 32.624147 \\
\hline C & 43.018410 & 39.781064 & 33.585565 \\
\hline 0 & 42.240849 & 39.745292 & 32.504234 \\
\hline $\mathrm{H}$ & 41.327308 & 40.015124 & 32.667855 \\
\hline C & 42.650243 & 40.442480 & 34.750620 \\
\hline $\mathrm{H}$ & 41.695016 & 40.930330 & 34.800948 \\
\hline C & 43.523195 & 40.492731 & 35.820778 \\
\hline $\mathrm{H}$ & 43.226295 & 41.043113 & 36.696323 \\
\hline C & 41.607664 & 35.984974 & 34.533187 \\
\hline $\mathrm{H}$ & 41.334818 & 35.625826 & 35.517820 \\
\hline $\mathrm{H}$ & 42.628741 & 36.344344 & 34.586554 \\
\hline C & 40.698892 & 37.146347 & 34.126626 \\
\hline $\mathrm{H}$ & 40.877830 & 37.986452 & 34.793320 \\
\hline $\mathrm{H}$ & 40.941507 & 37.533315 & 33.141227 \\
\hline C & 39.213900 & 36.875445 & 34.161196 \\
\hline 0 & 38.735728 & 35.831963 & 34.476121 \\
\hline 0 & 38.446812 & 37.887710 & 33.830552 \\
\hline $\mathrm{H}$ & 38.926353 & 38.685824 & 33.568122 \\
\hline N & 32.661527 & 46.816665 & 29.545867 \\
\hline $\mathrm{H}$ & 33.492188 & 46.266314 & 29.605034 \\
\hline C & 31.420980 & 46.130069 & 29.704097 \\
\hline C & 31.473586 & 44.743204 & 29.031992 \\
\hline$S$ & 31.806374 & 43.330820 & 30.128082 \\
\hline C & 33.584476 & 42.949045 & 30.391986 \\
\hline C & 34.595153 & 43.078989 & 29.374998 \\
\hline C & 35.914711 & 42.670226 & 29.507478 \\
\hline C & 36.679824 & 41.979844 & 30.491666 \\
\hline C & 36.334298 & 41.253782 & 31.668157 \\
\hline C & 37.304948 & 40.689274 & 32.451303 \\
\hline C & 38.710378 & 40.783029 & 32.157895 \\
\hline 0 & 39.572078 & 40.228458 & 32.897011 \\
\hline C & 39.047143 & 41.492606 & 30.962408 \\
\hline C & 38.067306 & 42.055845 & 30.185252 \\
\hline C & 38.276748 & 42.851494 & 28.925117 \\
\hline C & 36.862640 & 42.998834 & 28.355651 \\
\hline $\mathrm{H}$ & 36.675193 & 43.987647 & 27.949855 \\
\hline
\end{tabular}




$\begin{array}{llll}\text { H } & 36.700167 & 42.276888 & 27.559021 \\ \text { H } & 38.950242 & 42.353339 & 28.240477 \\ \text { H } & 38.711323 & 43.821007 & 29.144157 \\ \text { H } & 40.086990 & 41.590713 & 30.700441 \\ \text { H } & 37.016067 & 40.138253 & 33.327408 \\ \text { H } & 35.314204 & 41.109700 & 31.939497 \\ \text { H } & 34.314380 & 43.581679 & 28.468018 \\ \text { O } & 33.723103 & 42.494950 & 31.483546 \\ \text { H } & 30.496887 & 44.507414 & 28.631888 \\ \text { H } & 32.157105 & 44.771455 & 28.196304 \\ \text { H } & 30.642863 & 46.694927 & 29.203664 \\ \text { C } & 30.974633 & 46.071244 & 31.172223 \\ \text { O } & 29.841305 & 45.942158 & 31.507441 \\ \text { H } & 32.727264 & 47.789184 & 29.654279 \\ \text { H } & 31.746661 & 46.213104 & 31.874346 \\ \text { H } & 45.490258 & 40.012582 & 36.568056 \\ \text { H } & 41.569780 & 35.126039 & 33.825547\end{array}$

Point 3

$\begin{array}{llll}\text { C } & 44.754683 & 39.896693 & 35.784144 \\ \text { C } & 45.096418 & 39.204778 & 34.628861 \\ \text { H } & 46.039968 & 38.701536 & 34.541484 \\ \text { C } & 44.227799 & 39.154397 & 33.559626 \\ \text { H } & 44.501370 & 38.635794 & 32.661310 \\ \text { C } & 42.999901 & 39.802030 & 33.623933 \\ \text { O } & 42.215961 & 39.762339 & 32.547670 \\ \text { H } & 41.303808 & 40.034703 & 32.713658 \\ \text { C } & 42.641305 & 40.471695 & 34.787308 \\ \text { H } & 41.687024 & 40.961003 & 34.839808 \\ \text { C } & 43.520697 & 40.525107 & 35.851812 \\ \text { H } & 43.232066 & 41.081441 & 36.726287 \\ \mathrm{C} & 41.599178 & 36.004718 & 34.549004 \\ \text { H } & 41.327979 & 35.643963 & 35.533669 \\ \text { H } & 42.617829 & 36.371547 & 34.601499 \\ \mathrm{C} & 40.684961 & 37.161844 & 34.138656 \\ \text { H } & 40.859301 & 38.003780 & 34.803521 \\ \text { H } & 40.928795 & 37.550044 & 33.154464 \\ \mathrm{C} & 39.200837 & 36.886546 & 34.165378 \\ \mathrm{O} & 38.724193 & 35.838669 & 34.468593 \\ \mathrm{O} & 38.432497 & 37.900013 & 33.842298 \\ \mathrm{H} & 38.906659 & 38.705171 & 33.587375 \\ \mathrm{~N} & 32.674692 & 46.794870 & 29.558019 \\ \mathrm{H} & 33.507971 & 46.250082 & 29.641328 \\ \mathrm{C} & 31.435764 & 46.106224 & 29.702461\end{array}$




\begin{tabular}{|c|c|c|c|}
\hline C & 31.474664 & 44.733902 & 29.002939 \\
\hline$S$ & 31.832171 & 43.291416 & 30.048335 \\
\hline C & 33.630043 & 43.050357 & 30.375135 \\
\hline C & 34.629057 & 43.101151 & 29.339041 \\
\hline C & 35.948581 & 42.698095 & 29.486361 \\
\hline C & 36.700246 & 42.027641 & 30.492580 \\
\hline C & 36.333485 & 41.328471 & 31.678393 \\
\hline C & 37.289721 & 40.755607 & 32.472726 \\
\hline C & 38.698867 & 40.819275 & 32.185241 \\
\hline 0 & 39.543559 & 40.252642 & 32.936186 \\
\hline C & 39.057280 & 41.510940 & 30.985000 \\
\hline C & 38.089404 & 42.074333 & 30.191913 \\
\hline C & 38.316672 & 42.836494 & 28.914595 \\
\hline C & 36.906957 & 42.996837 & 28.335402 \\
\hline $\mathrm{H}$ & 36.737810 & 43.986754 & 27.923231 \\
\hline $\mathrm{H}$ & 36.738523 & 42.272128 & 27.542489 \\
\hline $\mathrm{H}$ & 38.983667 & 42.314043 & 28.241622 \\
\hline $\mathrm{H}$ & 38.766186 & 43.803808 & 29.115374 \\
\hline $\mathrm{H}$ & 40.100631 & 41.583403 & 30.728523 \\
\hline $\mathrm{H}$ & 36.988396 & 40.215812 & 33.351818 \\
\hline $\mathrm{H}$ & 35.309436 & 41.212306 & 31.950359 \\
\hline $\mathrm{H}$ & 34.335731 & 43.535242 & 28.401020 \\
\hline 0 & 33.804428 & 42.753909 & 31.513084 \\
\hline $\mathrm{H}$ & 30.487167 & 44.502808 & 28.628233 \\
\hline $\mathrm{H}$ & 32.132829 & 44.785289 & 28.148524 \\
\hline $\mathrm{H}$ & 30.658746 & 46.683928 & 29.214898 \\
\hline C & 30.982531 & 46.033891 & 31.167888 \\
\hline 0 & 29.849338 & 45.884543 & 31.496217 \\
\hline $\mathrm{H}$ & 32.733446 & 47.768381 & 29.663918 \\
\hline $\mathrm{H}$ & 31.744750 & 46.184482 & 31.877198 \\
\hline $\mathrm{H}$ & 45.488109 & 40.036237 & 36.591874 \\
\hline $\mathrm{H}$ & 41.567702 & 35.145644 & 33.841281 \\
\hline \multicolumn{4}{|c|}{ Point 4} \\
\hline C & 44.741670 & 39.922912 & 35.808539 \\
\hline C & 45.071883 & 39.217589 & 34.659384 \\
\hline $\mathrm{H}$ & 46.013081 & 38.707948 & 34.577168 \\
\hline C & 44.195769 & 39.161864 & 33.596162 \\
\hline $\mathrm{H}$ & 44.462342 & 38.630286 & 32.703383 \\
\hline C & 42.970130 & 39.814302 & 33.661765 \\
\hline 0 & 42.176356 & 39.767262 & 32.593730 \\
\hline $\mathrm{H}$ & 41.263990 & 40.041371 & 32.764229 \\
\hline C & 42.623463 & 40.498320 & 34.820424 \\
\hline $\mathrm{H}$ & 41.672174 & 40.993501 & 34.872042 \\
\hline
\end{tabular}




\begin{tabular}{|c|c|c|c|}
\hline C & 43.510751 & 40.557837 & 35.877711 \\
\hline $\mathrm{H}$ & 43.230134 & 41.120974 & 36.750114 \\
\hline C & 41.572460 & 36.008633 & 34.563800 \\
\hline $\mathrm{H}$ & 41.293215 & 35.643165 & 35.544548 \\
\hline $\mathrm{H}$ & 42.592330 & 36.371054 & 34.628844 \\
\hline C & 40.663189 & 37.168876 & 34.152025 \\
\hline $\mathrm{H}$ & 40.837594 & 38.008457 & 34.820231 \\
\hline $\mathrm{H}$ & 40.914182 & 37.558169 & 33.169329 \\
\hline C & 39.178387 & 36.895254 & 34.179335 \\
\hline 0 & 38.704588 & 35.842841 & 34.471593 \\
\hline 0 & 38.407849 & 37.911754 & 33.872655 \\
\hline $\mathrm{H}$ & 38.882600 & 38.720502 & 33.631515 \\
\hline $\mathrm{N}$ & 32.702356 & 46.778533 & 29.541093 \\
\hline $\mathrm{H}$ & 33.536116 & 46.239625 & 29.635153 \\
\hline C & 31.466799 & 46.087026 & 29.684897 \\
\hline C & 31.502254 & 44.735372 & 28.945183 \\
\hline$S$ & 31.845925 & 43.240262 & 29.925369 \\
\hline C & 33.647612 & 43.097749 & 30.315687 \\
\hline C & 34.632688 & 42.987240 & 29.274838 \\
\hline C & 35.945848 & 42.566765 & 29.439694 \\
\hline C & 36.699414 & 41.992242 & 30.498813 \\
\hline C & 36.340123 & 41.476391 & 31.779309 \\
\hline C & 37.289546 & 40.923092 & 32.597098 \\
\hline$C$ & 38.682860 & 40.829216 & 32.242495 \\
\hline 0 & 39.521211 & 40.276297 & 33.012505 \\
\hline$C$ & 39.031280 & 41.347295 & 30.957017 \\
\hline C & 38.067458 & 41.868236 & 30.132917 \\
\hline C & 38.281298 & 42.350978 & 28.725506 \\
\hline C & 36.874803 & 42.714082 & 28.236332 \\
\hline $\mathrm{H}$ & 36.834572 & 43.725512 & 27.844718 \\
\hline $\mathrm{H}$ & 36.554492 & 42.048221 & 27.439994 \\
\hline $\mathrm{H}$ & 38.719840 & 41.565630 & 28.121101 \\
\hline $\mathrm{H}$ & 38.953790 & 43.203460 & 28.692829 \\
\hline $\mathrm{H}$ & 40.056141 & 41.268484 & 30.636192 \\
\hline $\mathrm{H}$ & 36.995544 & 40.515768 & 33.547723 \\
\hline $\mathrm{H}$ & 35.325918 & 41.493198 & 32.109727 \\
\hline $\mathrm{H}$ & 34.317406 & 43.278505 & 28.289706 \\
\hline 0 & 33.838228 & 43.020159 & 31.486734 \\
\hline $\mathrm{H}$ & 30.519238 & 44.518732 & 28.550523 \\
\hline $\mathrm{H}$ & 32.172181 & 44.826810 & 28.103457 \\
\hline $\mathrm{H}$ & 30.687255 & 46.674719 & 29.212677 \\
\hline C & 31.019359 & 45.991756 & 31.151312 \\
\hline 0 & 29.885794 & 45.838936 & 31.471568 \\
\hline $\mathrm{H}$ & 32.756613 & 47.750636 & 29.651607 \\
\hline
\end{tabular}




$\begin{array}{llll}\mathrm{H} & 31.777069 & 46.142109 & 31.867908 \\ \mathrm{H} & 45.476835 & 40.064890 & 36.611604 \\ \mathrm{H} & 41.549198 & 35.151698 & 33.851493\end{array}$

Point 5

$\begin{array}{llll}\mathrm{C} & 44.722314 & 39.953493 & 35.839964 \\ \mathrm{C} & 45.039923 & 39.259709 & 34.680432 \\ \mathrm{H} & 45.984433 & 38.757320 & 34.584245 \\ \mathrm{C} & 44.151118 & 39.209315 & 33.626795 \\ \mathrm{H} & 44.408330 & 38.686619 & 32.725553 \\ \mathrm{C} & 42.923630 & 39.856793 & 33.712130 \\ \mathrm{O} & 42.114747 & 39.814169 & 32.654958 \\ \mathrm{H} & 41.200817 & 40.075462 & 32.840518 \\ \mathrm{C} & 42.590110 & 40.531156 & 34.880349 \\ \mathrm{H} & 41.637963 & 41.023183 & 34.947689 \\ \mathrm{C} & 43.489556 & 40.583025 & 35.928185 \\ \mathrm{H} & 43.218914 & 41.138691 & 36.808578 \\ \mathrm{C} & 41.564681 & 36.020862 & 34.589553 \\ \mathrm{H} & 41.296267 & 35.668918 & 35.578369 \\ \mathrm{H} & 42.582903 & 36.390999 & 34.638467 \\ \mathrm{C} & 40.647198 & 37.172479 & 34.170761 \\ \mathrm{H} & 40.839140 & 38.024842 & 34.816816 \\ \mathrm{H} & 40.873468 & 37.538540 & 33.173840 \\ \mathrm{C} & 39.163673 & 36.898921 & 34.238379 \\ \mathrm{O} & 38.702059 & 35.840874 & 34.529554 \\ \mathrm{O} & 38.383335 & 37.916020 & 33.962632 \\ \mathrm{H} & 38.846431 & 38.731034 & 33.716464 \\ \mathrm{~N} & 32.694363 & 46.817432 & 29.498984 \\ \mathrm{H} & 33.548361 & 46.310887 & 29.511279 \\ \mathrm{C} & 31.478264 & 46.086694 & 29.647792 \\ \mathrm{C} & 31.513396 & 44.741881 & 28.888629 \\ \mathrm{~S} & 31.793748 & 43.192795 & 29.819837 \\ \mathrm{C} & 33.608947 & 43.044565 & 30.195765 \\ \mathrm{C} & 34.463068 & 42.490816 & 29.182820 \\ \mathrm{C} & 35.755544 & 42.017405 & 29.354937 \\ \mathrm{C} & 36.567909 & 41.702747 & 30.474350 \\ \mathrm{C} & 36.275021 & 41.513288 & 31.854083 \\ \mathrm{C} & 37.243679 & 41.058247 & 32.711758 \\ \mathrm{C} & 38.595458 & 40.776375 & 32.299467 \\ \mathrm{C} & 39.449042 & 40.297780 & 33.105424 \\ \mathrm{C} & 38.882908 & 41.001547 & 30.915670 \\ \mathrm{C} & 37.896037 & 41.413067 & 30.058937 \\ \mathrm{C} & 36.5576047 & 41.593488 & 28.569851 \\ \mathrm{C} & 41.664448 & 28.107961\end{array}$




$\begin{array}{llll}\text { H } & 36.410224 & 42.376028 & 27.308379 \\ \text { H } & 36.230728 & 40.696991 & 27.740004 \\ \text { H } & 38.554896 & 40.779025 & 28.099203 \\ \text { H } & 38.540934 & 42.516443 & 28.333276 \\ \text { H } & 39.877678 & 40.789742 & 30.561350 \\ \text { H } & 36.992929 & 40.861244 & 33.738098 \\ \text { H } & 35.285621 & 41.675097 & 32.226327 \\ \text { H } & 34.053240 & 42.485105 & 28.190551 \\ \text { O } & 33.911403 & 43.366562 & 31.300468 \\ \text { H } & 30.536985 & 44.557591 & 28.464677 \\ \text { H } & 32.214555 & 44.822852 & 28.070365 \\ \text { H } & 30.679487 & 46.665281 & 29.195460 \\ \text { C } & 31.044107 & 45.951613 & 31.109825 \\ \text { O } & 29.918692 & 45.730808 & 31.431933 \\ \text { H } & 32.731761 & 47.785655 & 29.641025 \\ \text { H } & 31.798516 & 46.121831 & 31.821661 \\ \text { H } & 45.466186 & 40.090362 & 36.636528 \\ \text { H } & 41.537861 & 35.155354 & 33.888859\end{array}$

$\begin{array}{llll}\text { Point 6 } & & & \\ \text { C } & 44.729269 & 39.969340 & 35.845602 \\ \text { C } & 45.044456 & 39.304043 & 34.667671 \\ \text { H } & 45.996451 & 38.822709 & 34.546718 \\ \text { C } & 44.145371 & 39.263177 & 33.622488 \\ \text { H } & 44.402497 & 38.765498 & 32.707118 \\ \text { C } & 42.909893 & 39.892495 & 33.733327 \\ \text { O } & 42.092771 & 39.860827 & 32.681478 \\ \text { H } & 41.182556 & 40.126224 & 32.869754 \\ \text { C } & 42.580941 & 40.539532 & 34.917360 \\ \text { H } & 41.626285 & 41.023319 & 35.003999 \\ \text { C } & 43.489055 & 40.577950 & 35.958987 \\ \text { H } & 43.220517 & 41.111156 & 36.853705 \\ \text { C } & 41.540965 & 36.036912 & 34.587396 \\ \text { H } & 41.264341 & 35.695648 & 35.577427 \\ \text { H } & 42.557347 & 36.410978 & 34.640248 \\ \text { C } & 40.625020 & 37.187565 & 34.151645 \\ \text { H } & 40.808242 & 38.041637 & 34.798579 \\ \text { H } & 40.859129 & 37.550682 & 33.154158 \\ \text { C } & 39.140691 & 36.913202 & 34.200693 \\ \text { O } & 38.673699 & 35.853806 & 34.476071 \\ \text { O } & 38.360039 & 37.932808 & 33.928407 \\ \text { H } & 38.814476 & 38.754519 & 33.696051 \\ \text { N } & 32.668323 & 46.899049 & 29.471003 \\ \text { H } & 33.528494 & 46.409855 & 29.395932\end{array}$




\begin{tabular}{|c|c|c|c|}
\hline C & 31.460658 & 46.151742 & 29.620614 \\
\hline C & 31.476446 & 44.829960 & 28.824415 \\
\hline$S$ & 31.792221 & 43.237751 & 29.663422 \\
\hline C & 33.614043 & 43.169000 & 30.021906 \\
\hline C & 34.427038 & 42.444701 & 29.090069 \\
\hline C & 35.722463 & 41.980164 & 29.283569 \\
\hline C & 36.546976 & 41.759832 & 30.412354 \\
\hline C & 36.265901 & 41.679198 & 31.805219 \\
\hline C & 37.225759 & 41.238323 & 32.679986 \\
\hline C & 38.553905 & 40.851684 & 32.270012 \\
\hline 0 & 39.390790 & 40.367842 & 33.087349 \\
\hline C & 38.839347 & 40.998490 & 30.873545 \\
\hline C & 37.859886 & 41.395067 & 30.003437 \\
\hline C & 37.961007 & 41.483400 & 28.505625 \\
\hline C & 36.494839 & 41.483401 & 28.067135 \\
\hline $\mathrm{H}$ & 36.318037 & 42.078679 & 27.182002 \\
\hline $\mathrm{H}$ & 36.178937 & 40.470242 & 27.844292 \\
\hline $\mathrm{H}$ & 38.520459 & 40.659334 & 28.078443 \\
\hline $\mathrm{H}$ & 38.445105 & 42.412282 & 28.208921 \\
\hline $\mathrm{H}$ & 39.820484 & 40.723457 & 30.524080 \\
\hline $\mathrm{H}$ & 36.976804 & 41.114600 & 33.717296 \\
\hline $\mathrm{H}$ & 35.290141 & 41.919752 & 32.176259 \\
\hline $\mathrm{H}$ & 33.985954 & 42.303151 & 28.122046 \\
\hline 0 & 33.956818 & 43.695383 & 31.034409 \\
\hline $\mathrm{H}$ & 30.488505 & 44.661255 & 28.422674 \\
\hline $\mathrm{H}$ & 32.147044 & 44.938318 & 27.985712 \\
\hline $\mathrm{H}$ & 30.648239 & 46.738201 & 29.202790 \\
\hline C & 31.050288 & 45.967600 & 31.081096 \\
\hline 0 & 29.930576 & 45.717011 & 31.411177 \\
\hline $\mathrm{H}$ & 32.702041 & 47.862135 & 29.642659 \\
\hline $\mathrm{H}$ & 31.812331 & 46.126905 & 31.784357 \\
\hline $\mathrm{H}$ & 45.477143 & 40.094369 & 36.641583 \\
\hline $\mathrm{H}$ & 41.521132 & 35.162697 & 33.896657 \\
\hline \multicolumn{4}{|c|}{ Point 7} \\
\hline C & 44.725177 & 39.947739 & 35.821011 \\
\hline C & 45.031022 & 39.270154 & 34.647234 \\
\hline $\mathrm{H}$ & 45.972703 & 38.768800 & 34.525994 \\
\hline C & 44.132900 & 39.234129 & 33.601016 \\
\hline $\mathrm{H}$ & 44.383052 & 38.725794 & 32.689634 \\
\hline C & 42.908284 & 39.884687 & 33.703116 \\
\hline 0 & 42.093413 & 39.855921 & 32.647800 \\
\hline $\mathrm{H}$ & 41.191276 & 40.145054 & 32.832646 \\
\hline C & 42.589964 & 40.549247 & 34.879942 \\
\hline
\end{tabular}




\begin{tabular}{|c|c|c|c|}
\hline $\mathrm{H}$ & 41.645187 & 41.053950 & 34.959413 \\
\hline C & 43.496823 & 40.582442 & 35.923548 \\
\hline $\mathrm{H}$ & 43.232917 & 41.131208 & 36.810068 \\
\hline C & 41.510503 & 36.034622 & 34.565169 \\
\hline $\mathrm{H}$ & 41.212166 & 35.689602 & 35.548290 \\
\hline $\mathrm{H}$ & 42.525500 & 36.408508 & 34.638881 \\
\hline C & 40.605770 & 37.188113 & 34.115726 \\
\hline $\mathrm{H}$ & 40.769999 & 38.039459 & 34.770435 \\
\hline $\mathrm{H}$ & 40.870023 & 37.556159 & 33.128256 \\
\hline C & 39.119058 & 36.916346 & 34.116583 \\
\hline 0 & 38.640866 & 35.852038 & 34.347925 \\
\hline 0 & 38.347666 & 37.947156 & 33.852986 \\
\hline $\mathrm{H}$ & 38.808092 & 38.768271 & 33.640840 \\
\hline $\mathrm{N}$ & 32.673556 & 46.921674 & 29.450811 \\
\hline $\mathrm{H}$ & 33.533561 & 46.441643 & 29.345408 \\
\hline C & 31.469135 & 46.167540 & 29.608510 \\
\hline C & 31.462551 & 44.866569 & 28.781083 \\
\hline$S$ & 31.856283 & 43.242256 & 29.517190 \\
\hline C & 33.684962 & 43.223876 & 29.815148 \\
\hline C & 34.447083 & 42.365701 & 28.962253 \\
\hline C & 35.749320 & 41.919867 & 29.165183 \\
\hline C & 36.578427 & 41.775749 & 30.300223 \\
\hline C & 36.297743 & 41.776531 & 31.695904 \\
\hline C & 37.247446 & 41.359405 & 32.593220 \\
\hline C & 38.561069 & 40.905195 & 32.200546 \\
\hline 0 & 39.378893 & 40.427646 & 33.036593 \\
\hline C & 38.852977 & 40.984550 & 30.798377 \\
\hline C & 37.883945 & 41.363216 & 29.910196 \\
\hline C & 37.979605 & 41.386323 & 28.409427 \\
\hline C & 36.510852 & 41.337109 & 27.981428 \\
\hline $\mathrm{H}$ & 36.316726 & 41.849806 & 27.048168 \\
\hline $\mathrm{H}$ & 36.211078 & 40.303730 & 27.847120 \\
\hline $\mathrm{H}$ & 38.558354 & 40.564009 & 28.006966 \\
\hline $\mathrm{H}$ & 38.438209 & 42.315979 & 28.078582 \\
\hline $\mathrm{H}$ & 39.827485 & 40.670971 & 30.462960 \\
\hline $\mathrm{H}$ & 36.996238 & 41.288487 & 33.635022 \\
\hline $\mathrm{H}$ & 35.330203 & 42.070779 & 32.053560 \\
\hline $\mathrm{H}$ & 33.977894 & 42.131906 & 28.025662 \\
\hline 0 & 34.078784 & 43.902132 & 30.713868 \\
\hline $\mathrm{H}$ & 30.451615 & 44.686200 & 28.444884 \\
\hline $\mathrm{H}$ & 32.078839 & 45.013209 & 27.907026 \\
\hline $\mathrm{H}$ & 30.654439 & 46.759934 & 29.203403 \\
\hline C & 31.074126 & 45.950206 & 31.069981 \\
\hline 0 & 29.961735 & 45.666169 & 31.392429 \\
\hline
\end{tabular}




$\begin{array}{llll}\mathrm{H} & 32.710987 & 47.879047 & 29.648135 \\ \mathrm{H} & 31.831956 & 46.120577 & 31.778890 \\ \mathrm{H} & 45.478687 & 40.067846 & 36.614974 \\ \mathrm{H} & 41.504806 & 35.163389 & 33.870856\end{array}$

Point 8

\begin{tabular}{|c|c|c|c|}
\hline C & 44.724737 & 39.930356 & 35.774382 \\
\hline C & 45.043583 & 39.261886 & 34.598652 \\
\hline 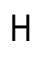 & 45.988670 & 38.766533 & 34.482608 \\
\hline ; & 44.153976 & 39.226130 & 33.545761 \\
\hline $\mathrm{H}$ & 44.415534 & 38.727183 & 32.632419 \\
\hline C & 42.926404 & 39.871767 & 33.642034 \\
\hline J & 42.120244 & 39.846848 & 32.579205 \\
\hline H & 41.223159 & 40.152021 & 32.756081 \\
\hline C & 42.593968 & 40.527472 & 34.819798 \\
\hline $\mathrm{H}$ & 41.646080 & 41.027489 & 34.893640 \\
\hline C & 43.492461 & 40.557942 & 35.870859 \\
\hline $\mathrm{H}$ & 43.221190 & 41.099825 & 36.759567 \\
\hline C & 41.505221 & 36.003847 & 34.501359 \\
\hline $\mathrm{H}$ & 41.187375 & 35.658671 & 35.478388 \\
\hline $\mathrm{H}$ & 42.520017 & 36.373484 & 34.595223 \\
\hline C & 40.609885 & 37.160875 & 34.041172 \\
\hline $\mathrm{H}$ & 40.764735 & 38.008562 & 34.702997 \\
\hline H & 40.889015 & 37.532594 & 33.059182 \\
\hline C & 39.121998 & 36.891997 & 34.018386 \\
\hline 0 & 38.633706 & 35.827284 & 34.219812 \\
\hline 0 & 38.357829 & 37.933099 & 33.765537 \\
\hline $\mathrm{H}$ & 38.825825 & 38.750312 & 33.568432 \\
\hline $\mathrm{N}$ & 32.645780 & 46.934656 & 29.469072 \\
\hline $\mathrm{H}$ & 33.506337 & 46.453122 & 29.373813 \\
\hline C & 31.447753 & 46.173302 & 29.630456 \\
\hline C & 31.459223 & 44.876977 & 28.793553 \\
\hline$S$ & 31.904226 & 43.251943 & 29.494752 \\
\hline C & 33.742073 & 43.278622 & 29.682603 \\
\hline$C$ & 34.437148 & 42.300683 & 28.909774 \\
\hline C & 35.750358 & 41.873010 & 29.089898 \\
\hline C & 36.600590 & 41.788111 & 30.211267 \\
\hline C & 36.333284 & 41.841210 & 31.608489 \\
\hline C & 37.281137 & 41.428836 & 32.508314 \\
\hline C & 38.581786 & 40.935418 & 32.116659 \\
\hline 0 & 39.398853 & 40.471490 & 32.959346 \\
\hline C & 38.861146 & 40.959606 & 30.708706 \\
\hline C & 37.892446 & 41.333742 & 29.820309 \\
\hline C & 37.963039 & 41.308626 & 28.318833 \\
\hline
\end{tabular}




$\begin{array}{llll}\mathrm{C} & 36.486806 & 41.238898 & 27.918079 \\ \mathrm{H} & 36.276770 & 41.718769 & 26.970529 \\ \mathrm{H} & 36.190173 & 40.199870 & 27.830022 \\ \mathrm{H} & 38.545317 & 40.482761 & 27.929778 \\ \mathrm{H} & 38.402655 & 42.236080 & 27.958083 \\ \mathrm{H} & 39.823856 & 40.608290 & 30.376288 \\ \mathrm{H} & 37.041946 & 41.400527 & 33.554281 \\ \mathrm{H} & 35.376322 & 42.170649 & 31.966856 \\ \mathrm{H} & 33.908254 & 41.959244 & 28.040620 \\ \mathrm{O} & 34.204909 & 44.092138 & 30.428400 \\ \mathrm{H} & 30.451643 & 44.683621 & 28.454010 \\ \mathrm{H} & 32.064781 & 45.046304 & 27.915374 \\ \mathrm{H} & 30.620552 & 46.756536 & 29.236072 \\ \mathrm{C} & 31.067601 & 45.950414 & 31.092175 \\ \mathrm{O} & 29.971391 & 45.628278 & 31.423705 \\ \mathrm{H} & 32.683055 & 47.894395 & 29.654297 \\ \mathrm{H} & 31.822038 & 46.156884 & 31.795783 \\ \mathrm{H} & 45.475298 & 40.058669 & 36.569745 \\ \mathrm{H} & 41.508340 & 35.133669 & 33.806794\end{array}$

Point 9

$\begin{array}{llll}\text { C } & 44.735413 & 39.938195 & 35.775601 \\ \mathrm{C} & 45.041881 & 39.261858 & 34.601398 \\ \mathrm{H} & 45.985010 & 38.763393 & 34.482299 \\ \mathrm{C} & 44.143439 & 39.224376 & 33.555269 \\ \mathrm{H} & 44.396794 & 38.719093 & 32.643193 \\ \mathrm{C} & 42.917190 & 39.871045 & 33.660745 \\ \mathrm{O} & 42.098701 & 39.839293 & 32.607957 \\ \mathrm{H} & 41.204103 & 40.148963 & 32.793491 \\ \mathrm{C} & 42.596088 & 40.531984 & 34.838549 \\ \mathrm{H} & 41.649158 & 41.032813 & 34.917401 \\ \mathrm{C} & 43.504571 & 40.566363 & 35.880650 \\ \mathrm{H} & 43.244136 & 41.113682 & 36.769333 \\ \mathrm{C} & 41.495793 & 35.993901 & 34.508401 \\ \mathrm{H} & 41.169063 & 35.642890 & 35.480278 \\ \mathrm{H} & 42.508917 & 36.364281 & 34.616266 \\ \mathrm{C} & 40.604312 & 37.154368 & 34.046535 \\ \mathrm{H} & 40.750950 & 37.996192 & 34.717711 \\ \mathrm{H} & 40.895283 & 37.534630 & 33.070983 \\ \mathrm{C} & 39.117863 & 36.884756 & 33.998352 \\ \mathrm{O} & 38.627471 & 35.816043 & 34.172686 \\ \mathrm{O} & 38.356705 & 37.928616 & 33.751000 \\ \mathrm{H} & 38.824317 & 38.752758 & 33.579946 \\ \mathrm{~N} & 32.625332 & 46.949275 & 29.508589\end{array}$




$\begin{array}{llll}\text { H } & 33.484235 & 46.456279 & 29.449143 \\ \text { C } & 31.425752 & 46.198435 & 29.674941 \\ \text { C } & 31.422962 & 44.901112 & 28.846873 \\ \text { S } & 31.973355 & 43.298859 & 29.523275 \\ \text { C } & 33.807184 & 43.408496 & 29.635313 \\ \text { C } & 34.507450 & 42.384230 & 28.919803 \\ \text { C } & 35.815199 & 41.947264 & 29.120034 \\ \text { C } & 36.650435 & 41.851086 & 30.253770 \\ \text { C } & 36.358848 & 41.880255 & 31.646063 \\ \text { C } & 37.285125 & 41.432190 & 32.552356 \\ \text { C } & 38.595325 & 40.954677 & 32.175437 \\ \text { O } & 39.397825 & 40.475090 & 33.022187 \\ \text { C } & 38.897472 & 40.999823 & 30.773627 \\ \text { C } & 37.946882 & 41.399097 & 29.876004 \\ \text { C } & 38.033844 & 41.379332 & 28.376060 \\ \text { C } & 36.561314 & 41.293639 & 27.964638 \\ \text { H } & 36.355301 & 41.752998 & 27.006464 \\ \text { H } & 36.266281 & 40.251592 & 27.894651 \\ \text { H } & 38.626117 & 40.557076 & 27.995038 \\ \text { H } & 38.466847 & 42.310207 & 28.016058 \\ \text { H } & 39.865314 & 40.654283 & 30.451579 \\ \text { H } & 37.032145 & 41.395197 & 33.593843 \\ \text { H } & 35.387317 & 42.182328 & 31.991477 \\ \text { H } & 33.985883 & 42.009475 & 28.059852 \\ \text { O } & 34.266329 & 44.306342 & 30.277858 \\ \text { H } & 30.402696 & 44.667748 & 28.577763 \\ \text { H } & 31.965386 & 45.093374 & 27.933424 \\ \text { H } & 30.600837 & 46.787162 & 29.284657 \\ \text { C } & 31.056833 & 45.972681 & 31.138123 \\ \text { O } & 29.965457 & 45.637540 & 31.481621 \\ \text { H } & 32.664671 & 47.912841 & 29.676295 \\ \text { H } & 31.816196 & 46.188236 & 31.832377 \\ \text { H } & 45.486873 & 40.059164 & 36.569987 \\ \text { H } & 41.508117 & 35.126722 & 33.810464\end{array}$

The QM(CASSCF)/MM optimized minimum-energy structures and conical intersections in and between the $S_{0}$ and $S_{1}$ states of the single-bond-rotation locked PYP chromophore in R52A mutated protein.

$\begin{array}{llll}\text { SO-MIN } & & & \\ \text { C } & 44.815587 & 39.813600 & 35.776311 \\ \text { C } & 45.162855 & 39.071207 & 34.656647\end{array}$




\begin{tabular}{|c|c|c|c|}
\hline $\mathrm{H}$ & 46.106341 & 38.561231 & 34.608366 \\
\hline C & 44.310261 & 38.984006 & 33.576941 \\
\hline $\mathrm{H}$ & 44.587692 & 38.423634 & 32.704190 \\
\hline C & 43.085083 & 39.641069 & 33.595425 \\
\hline 0 & 42.303529 & 39.549203 & 32.528582 \\
\hline $\mathrm{H}$ & 41.374292 & 39.789656 & 32.711258 \\
\hline C & 42.724113 & 40.371990 & 34.724285 \\
\hline $\mathrm{H}$ & 41.769658 & 40.863286 & 34.738359 \\
\hline$C$ & 43.589543 & 40.461785 & 35.795829 \\
\hline $\mathrm{H}$ & 43.298216 & 41.052378 & 36.647592 \\
\hline C & 41.732793 & 35.911191 & 34.615091 \\
\hline $\mathrm{H}$ & 41.512319 & 35.546615 & 35.611377 \\
\hline $\mathrm{H}$ & 42.768406 & 36.232137 & 34.615261 \\
\hline C & 40.836937 & 37.099785 & 34.293733 \\
\hline $\mathrm{H}$ & 41.115424 & 37.937728 & 34.927471 \\
\hline $\mathrm{H}$ & 40.970711 & 37.466696 & 33.283070 \\
\hline C & 39.365616 & 36.876414 & 34.532791 \\
\hline 0 & 38.924704 & 35.887581 & 35.033483 \\
\hline 0 & 38.588875 & 37.862452 & 34.173817 \\
\hline $\mathrm{H}$ & 39.072778 & 38.628290 & 33.796143 \\
\hline $\mathrm{N}$ & 32.511552 & 46.909734 & 29.277196 \\
\hline $\mathrm{H}$ & 33.308026 & 46.369274 & 29.042248 \\
\hline C & 31.282419 & 46.237730 & 29.512502 \\
\hline C & 31.292929 & 44.819148 & 28.933500 \\
\hline$S$ & 32.173364 & 43.561484 & 29.917766 \\
\hline C & 33.862122 & 43.524190 & 29.214998 \\
\hline C & 34.708414 & 42.685870 & 30.027495 \\
\hline C & 36.039637 & 42.415229 & 29.826244 \\
\hline C & 36.878468 & 41.745619 & 30.764800 \\
\hline C & 36.554888 & 41.218720 & 32.024330 \\
\hline C & 37.535392 & 40.646213 & 32.787181 \\
\hline C & 38.909282 & 40.570750 & 32.362814 \\
\hline 0 & 39.760532 & 39.984352 & 33.115662 \\
\hline C & 39.213204 & 41.116477 & 31.096669 \\
\hline C & 38.209713 & 41.692160 & 30.319554 \\
\hline C & 38.321600 & 42.331923 & 28.956455 \\
\hline$C$ & 36.872966 & 42.742718 & 28.599852 \\
\hline $\mathrm{H}$ & 36.790480 & 43.783993 & 28.320369 \\
\hline $\mathrm{H}$ & 36.494094 & 42.169563 & 27.759798 \\
\hline $\mathrm{H}$ & 38.730107 & 41.645998 & 28.220871 \\
\hline $\mathrm{H}$ & 38.979399 & 43.194916 & 28.975063 \\
\hline $\mathrm{H}$ & 40.227558 & 41.054744 & 30.742395 \\
\hline $\mathrm{H}$ & 37.291168 & 40.219911 & 33.743662 \\
\hline $\mathrm{H}$ & 35.546495 & 41.245738 & 32.395129 \\
\hline
\end{tabular}




$\begin{array}{llll}\mathrm{H} & 34.252843 & 42.316711 & 30.925418 \\ \mathrm{O} & 34.123877 & 44.140693 & 28.221306 \\ \mathrm{H} & 30.274413 & 44.463907 & 28.891101 \\ \mathrm{H} & 31.701189 & 44.837910 & 27.933165 \\ \mathrm{H} & 30.454302 & 46.745733 & 29.025789 \\ \mathrm{C} & 30.867440 & 46.188799 & 30.980716 \\ \mathrm{O} & 29.736426 & 46.040678 & 31.311743 \\ \mathrm{H} & 32.641384 & 47.857641 & 29.485036 \\ \mathrm{H} & 31.642490 & 46.304786 & 31.685598 \\ \mathrm{H} & 45.539073 & 39.973753 & 36.588519 \\ \mathrm{H} & 41.619581 & 35.065076 & 33.902261\end{array}$

S0-cisT-MIN

$\begin{array}{llll}\text { C } & 44.683102 & 39.848855 & 35.774645 \\ \text { C } & 44.992027 & 39.116041 & 34.634144 \\ \text { H } & 45.920526 & 38.581788 & 34.551106 \\ \text { C } & 44.111377 & 39.070615 & 33.577105 \\ \text { H } & 44.359814 & 38.522458 & 32.689455 \\ \text { C } & 42.907581 & 39.771212 & 33.627427 \\ \text { O } & 42.120551 & 39.730794 & 32.566722 \\ \text { H } & 41.211162 & 40.055353 & 32.700419 \\ \mathrm{C} & 42.588312 & 40.488400 & 34.777082 \\ \text { H } & 41.655013 & 41.017996 & 34.817357 \\ \mathrm{C} & 43.477487 & 40.532389 & 35.832404 \\ \mathrm{H} & 43.221163 & 41.121958 & 36.696067 \\ \mathrm{C} & 41.412636 & 35.941960 & 34.457678 \\ \mathrm{H} & 41.046206 & 35.612256 & 35.423883 \\ \mathrm{H} & 42.435935 & 36.269794 & 34.602757 \\ \mathrm{C} & 40.579394 & 37.130591 & 33.960738 \\ \mathrm{H} & 40.743762 & 37.970205 & 34.630541 \\ \mathrm{H} & 40.910109 & 37.496348 & 32.992879 \\ \mathrm{C} & 39.083638 & 36.929124 & 33.875556 \\ \mathrm{O} & 38.540975 & 35.883146 & 34.061091 \\ \mathrm{O} & 38.385119 & 37.998633 & 33.593002 \\ \mathrm{H} & 38.890970 & 38.806879 & 33.389791 \\ \mathrm{~N} & 32.680671 & 46.617204 & 29.393310 \\ \mathrm{H} & 33.531008 & 46.106495 & 29.435300 \\ \mathrm{C} & 31.482791 & 45.901455 & 29.611236 \\ \mathrm{C} & 31.393884 & 44.597019 & 28.802015 \\ \mathrm{~S} & 32.126886 & 43.006566 & 29.362558 \\ \mathrm{C} & 33.967170 & 43.192679 & 29.426157 \\ \mathrm{C} & 34.663421 & 42.148851 & 28.731054 \\ \mathrm{C} & 35.979000 & 41.729154 & 28.904663 \\ \mathrm{C} & 36.811993 & 41.663071 & 30.041269\end{array}$




$\begin{array}{llll}\text { C } & 36.492871 & 41.702312 & 31.421052 \\ \text { C } & 37.422464 & 41.298939 & 32.345951 \\ \text { C } & 38.741137 & 40.849562 & 31.984370 \\ \text { O } & 39.526373 & 40.357172 & 32.867856 \\ \text { C } & 39.073853 & 40.892338 & 30.594294 \\ \text { C } & 38.125471 & 41.261961 & 29.669856 \\ \text { C } & 38.211943 & 41.237097 & 28.162817 \\ \text { C } & 36.737765 & 41.072559 & 27.763406 \\ \text { H } & 36.513653 & 41.476664 & 26.785639 \\ \text { H } & 36.490819 & 40.015417 & 27.752288 \\ \text { H } & 38.841813 & 40.454017 & 27.763159 \\ \text { H } & 38.588036 & 42.181727 & 27.778908 \\ \text { H } & 40.050744 & 40.558350 & 30.290011 \\ \text { H } & 37.156590 & 41.257493 & 33.384778 \\ \text { H } & 35.503548 & 41.972330 & 31.742064 \\ \text { H } & 34.145652 & 41.787602 & 27.863227 \\ \text { O } & 34.413369 & 44.129337 & 30.019093 \\ \text { H } & 30.352495 & 44.313801 & 28.734187 \\ \text { H } & 31.749924 & 44.807154 & 27.802677 \\ \text { H } & 30.652945 & 46.501402 & 29.245822 \\ \text { C } & 31.089420 & 45.724337 & 31.068479 \\ \text { O } & 30.006064 & 45.359269 & 31.396597 \\ \text { H } & 32.734469 & 47.580110 & 29.559513 \\ \text { H } & 31.810192 & 46.007206 & 31.787522 \\ \text { H } & 45.439143 & 40.005154 & 36.559394 \\ \text { H } & 41.416771 & 35.063638 & 33.773845 \\ & & & \end{array}$

$\begin{array}{llll}\text { S0-cisP-MIN } & & & \\ \text { C } & 44.768347 & 39.873593 & 35.817751 \\ \text { C } & 45.080864 & 39.177312 & 34.657935 \\ \text { H } & 46.024882 & 38.674657 & 34.557739 \\ \text { C } & 44.191304 & 39.124120 & 33.605149 \\ \text { H } & 44.445687 & 38.607466 & 32.698554 \\ \text { C } & 42.967816 & 39.780149 & 33.685547 \\ \text { O } & 42.152872 & 39.726039 & 32.635297 \\ \text { H } & 41.237365 & 39.983556 & 32.840828 \\ \text { C } & 42.646881 & 40.475012 & 34.846566 \\ \text { H } & 41.699221 & 40.976465 & 34.908346 \\ \text { C } & 43.546236 & 40.524889 & 35.894073 \\ \text { H } & 43.283221 & 41.087747 & 36.772550 \\ \text { C } & 41.674885 & 35.946685 & 34.571316 \\ \text { H } & 41.433750 & 35.589177 & 35.566322 \\ \text { H } & 42.698608 & 36.304479 & 34.596086 \\ \text { C } & 40.755484 & 37.104463 & 34.194243\end{array}$




\begin{tabular}{|c|c|c|c|}
\hline $\mathrm{H}$ & 40.956303 & 37.947945 & 34.848100 \\
\hline $\mathrm{H}$ & 40.954362 & 37.480482 & 33.197028 \\
\hline C & 39.273902 & 36.829254 & 34.289664 \\
\hline 0 & 38.815652 & 35.783842 & 34.629903 \\
\hline 0 & 38.498573 & 37.835598 & 33.978048 \\
\hline $\mathrm{H}$ & 38.965545 & 38.655984 & 33.729848 \\
\hline $\mathrm{N}$ & 32.629112 & 46.895081 & 29.529979 \\
\hline $\mathrm{H}$ & 33.483832 & 46.394197 & 29.496608 \\
\hline C & 31.432018 & 46.142359 & 29.733829 \\
\hline C & 31.610040 & 44.695761 & 29.197213 \\
\hline S & 31.804020 & 43.362282 & 30.447027 \\
\hline C & 33.496601 & 42.631832 & 30.619202 \\
\hline C & 34.560687 & 42.926346 & 29.694049 \\
\hline C & 35.881465 & 42.532418 & 29.796957 \\
\hline C & 36.663282 & 41.859660 & 30.779471 \\
\hline C & 36.323791 & 41.203216 & 31.994308 \\
\hline C & 37.301309 & 40.653919 & 32.781195 \\
\hline C & 38.696962 & 40.706822 & 32.447030 \\
\hline 0 & 39.566754 & 40.149775 & 33.189971 \\
\hline C & 39.026617 & 41.366063 & 31.224542 \\
\hline C & 38.040039 & 41.907710 & 30.435002 \\
\hline C & 38.237063 & 42.666003 & 29.147063 \\
\hline C & 36.806757 & 42.871071 & 28.630739 \\
\hline $\mathrm{H}$ & 36.634264 & 43.871704 & 28.249422 \\
\hline $\mathrm{H}$ & 36.590463 & 42.179510 & 27.820927 \\
\hline $\mathrm{H}$ & 38.857197 & 42.126307 & 28.439275 \\
\hline $\mathrm{H}$ & 38.724232 & 43.616556 & 29.338206 \\
\hline $\mathrm{H}$ & 40.061524 & 41.422351 & 30.932913 \\
\hline $\mathrm{H}$ & 37.031492 & 40.150546 & 33.691433 \\
\hline $\mathrm{H}$ & 35.304523 & 41.103570 & 32.288349 \\
\hline $\mathrm{H}$ & 34.287328 & 43.508602 & 28.835406 \\
\hline 0 & 33.489218 & 41.883507 & 31.544483 \\
\hline $\mathrm{H}$ & 30.711015 & 44.404748 & 28.674284 \\
\hline $\mathrm{H}$ & 32.417774 & 44.678054 & 28.480390 \\
\hline $\mathrm{H}$ & 30.601923 & 46.585012 & 29.191500 \\
\hline C & 31.002211 & 46.166787 & 31.199032 \\
\hline 0 & 29.865856 & 46.103816 & 31.540017 \\
\hline $\mathrm{H}$ & 32.682092 & 47.863269 & 29.668437 \\
\hline $\mathrm{H}$ & 31.789205 & 46.289013 & 31.891141 \\
\hline $\mathrm{H}$ & 45.521956 & 40.017318 & 36.604925 \\
\hline $\mathrm{H}$ & 41.611088 & 35.089366 & 33.865685 \\
\hline \multicolumn{4}{|c|}{ S1-MIN } \\
\hline C & 44.849623 & 39.795177 & 35.795743 \\
\hline
\end{tabular}




\begin{tabular}{|c|c|c|c|}
\hline C & 45.217434 & 39.066228 & 34.674585 \\
\hline $\mathrm{H}$ & 46.165138 & 38.567256 & 34.636811 \\
\hline C & 44.383769 & 38.975508 & 33.579666 \\
\hline H & 44.683363 & 38.436503 & 32.700008 \\
\hline C & 43.161914 & 39.630534 & 33.586219 \\
\hline 0 & 42.392972 & 39.540034 & 32.495773 \\
\hline $\mathrm{H}$ & 41.495371 & 39.822787 & 32.668185 \\
\hline C & 42.778068 & 40.355251 & 34.706316 \\
\hline $\mathrm{H}$ & 41.826245 & 40.852593 & 34.709295 \\
\hline C & 43.623636 & 40.440594 & 35.795729 \\
\hline $\mathrm{H}$ & 43.314267 & 41.023754 & 36.645575 \\
\hline C & 41.757540 & 35.940458 & 34.611740 \\
\hline $\mathrm{H}$ & 41.574935 & 35.588586 & 35.620421 \\
\hline $\mathrm{H}$ & 42.785029 & 36.283886 & 34.573276 \\
\hline C & 40.820440 & 37.100839 & 34.304713 \\
\hline $\mathrm{H}$ & 41.093971 & 37.956236 & 34.915972 \\
\hline $\mathrm{H}$ & 40.900033 & 37.446170 & 33.279780 \\
\hline C & 39.367688 & 36.830456 & 34.603189 \\
\hline 0 & 38.969442 & 35.840828 & 35.121709 \\
\hline 0 & 38.538233 & 37.793842 & 34.261326 \\
\hline $\mathrm{H}$ & 38.988092 & 38.553692 & 33.887799 \\
\hline $\mathrm{N}$ & 32.477829 & 46.942891 & 29.352220 \\
\hline $\mathrm{H}$ & 33.262731 & 46.410818 & 29.057831 \\
\hline C & 31.240365 & 46.267205 & 29.543975 \\
\hline C & 31.262937 & 44.859388 & 28.933142 \\
\hline$S$ & 32.122740 & 43.554422 & 29.876797 \\
\hline C & 33.869118 & 43.616093 & 29.228282 \\
\hline C & 34.682952 & 42.697755 & 29.918212 \\
\hline C & 36.055406 & 42.434596 & 29.689447 \\
\hline C & 36.863594 & 41.742255 & 30.667216 \\
\hline C & 36.474076 & 41.156115 & 31.898581 \\
\hline C & 37.435143 & 40.584983 & 32.709899 \\
\hline C & 38.825657 & 40.607086 & 32.360421 \\
\hline 0 & 39.692824 & 40.064217 & 33.096045 \\
\hline C & 39.193291 & 41.207480 & 31.095736 \\
\hline C & 38.227191 & 41.749697 & 30.281478 \\
\hline C & 38.376250 & 42.453652 & 28.949718 \\
\hline C & 36.924252 & 42.772964 & 28.501290 \\
\hline $\mathrm{H}$ & 36.810169 & 43.800222 & 28.180010 \\
\hline $\mathrm{H}$ & 36.644227 & 42.149754 & 27.652799 \\
\hline $\mathrm{H}$ & 38.899692 & 41.837347 & 28.225586 \\
\hline $\mathrm{H}$ & 38.959017 & 43.362171 & 29.067101 \\
\hline $\mathrm{H}$ & 40.233142 & 41.198623 & 30.819093 \\
\hline $\mathrm{H}$ & 37.163569 & 40.133873 & 33.646159 \\
\hline
\end{tabular}




$\begin{array}{llll}\mathrm{H} & 35.440944 & 41.113375 & 32.179278 \\ \mathrm{H} & 34.225063 & 42.204314 & 30.752950 \\ \mathrm{O} & 34.127123 & 44.359940 & 28.290421 \\ \mathrm{H} & 30.243485 & 44.510080 & 28.864380 \\ \mathrm{H} & 31.691314 & 44.905564 & 27.942216 \\ \mathrm{H} & 30.411064 & 46.786078 & 29.067669 \\ \mathrm{C} & 30.854453 & 46.182785 & 31.011306 \\ \mathrm{O} & 29.730472 & 46.017468 & 31.372271 \\ \mathrm{H} & 32.600186 & 47.895024 & 29.547130 \\ \mathrm{H} & 31.650598 & 46.291754 & 31.692701 \\ \mathrm{H} & 45.561937 & 39.961706 & 36.615770 \\ \mathrm{H} & 41.634494 & 35.085621 & 33.911741\end{array}$

$\begin{array}{llll}\text { S1-CT1-MIN } & & & \\ \text { C } & 44.754759 & 39.852884 & 35.852546 \\ \text { C } & 45.056891 & 39.108971 & 34.718265 \\ \text { H } & 45.981929 & 38.569415 & 34.637084 \\ \text { C } & 44.165305 & 39.051432 & 33.670085 \\ \text { H } & 44.404420 & 38.489576 & 32.788522 \\ \text { C } & 42.952562 & 39.733065 & 33.733825 \\ \text { O } & 42.140775 & 39.673691 & 32.692841 \\ \text { H } & 41.214685 & 39.939496 & 32.886436 \\ \text { C } & 42.641409 & 40.460046 & 34.880977 \\ \text { H } & 41.700931 & 40.974642 & 34.930653 \\ \text { C } & 43.543378 & 40.527083 & 35.921752 \\ \text { H } & 43.290974 & 41.120839 & 36.783462 \\ \text { C } & 41.446708 & 35.901698 & 34.487590 \\ \text { H } & 41.116186 & 35.523185 & 35.448365 \\ \text { H } & 42.464965 & 36.251478 & 34.618811 \\ \text { C } & 40.571636 & 37.077903 & 34.052785 \\ \text { H } & 40.729729 & 37.903043 & 34.741211 \\ \text { H } & 40.869181 & 37.485809 & 33.091568 \\ \text { C } & 39.080821 & 36.837473 & 33.994519 \\ \text { O } & 38.572109 & 35.767674 & 34.152048 \\ \text { O } & 38.354119 & 37.897133 & 33.762142 \\ \text { H } & 38.869550 & 38.720341 & 33.598974 \\ \text { N } & 32.599519 & 46.825089 & 29.336463 \\ \text { H } & 33.433481 & 46.306132 & 29.197362 \\ \text { C } & 31.419965 & 46.094971 & 29.613584 \\ \text { C } & 31.457357 & 44.696367 & 28.982432 \\ \text { S } & 32.368189 & 43.342645 & 29.816537 \\ \text { C } & 34.074497 & 43.500438 & 29.230779 \\ \text { C } & 34.812694 & 42.246403 & 29.186640 \\ \text { C } & 36.264297 & 42.163049 & 29.279403\end{array}$




\begin{tabular}{|c|c|c|c|}
\hline C & 36.974428 & 41.785054 & 30.429406 \\
\hline C & 36.527560 & 41.455137 & 31.735574 \\
\hline C & 37.431406 & 40.952886 & 32.650489 \\
\hline C & 38.806967 & 40.753302 & 32.352800 \\
\hline 0 & 39.602688 & 40.168993 & 33.207708 \\
\hline C & 39.247311 & 41.133864 & 31.051644 \\
\hline C & 38.355622 & 41.630845 & 30.126007 \\
\hline C & 38.606741 & 42.022230 & 28.686909 \\
\hline C & 37.193046 & 42.204579 & 28.081304 \\
\hline $\mathrm{H}$ & 37.115599 & 43.149113 & 27.553508 \\
\hline $\mathrm{H}$ & 36.956027 & 41.419087 & 27.365590 \\
\hline $\mathrm{H}$ & 39.181236 & 41.275831 & 28.151284 \\
\hline $\mathrm{H}$ & 39.163104 & 42.953345 & 28.631663 \\
\hline $\mathrm{H}$ & 40.279363 & 40.973088 & 30.790726 \\
\hline $\mathrm{H}$ & 37.088137 & 40.654409 & 33.625354 \\
\hline $\mathrm{H}$ & 35.492233 & 41.549744 & 32.010047 \\
\hline $\mathrm{H}$ & 34.228621 & 41.366404 & 28.979550 \\
\hline 0 & 34.532873 & 44.564456 & 28.905830 \\
\hline $\mathrm{H}$ & 30.449615 & 44.304653 & 28.954638 \\
\hline $\mathrm{H}$ & 31.827912 & 44.790793 & 27.970221 \\
\hline $\mathrm{H}$ & 30.560110 & 46.586948 & 29.167683 \\
\hline C & 31.009207 & 46.019592 & 31.084196 \\
\hline 0 & 29.892379 & 45.803039 & 31.417898 \\
\hline $\mathrm{H}$ & 32.688815 & 47.775264 & 29.552711 \\
\hline $\mathrm{H}$ & 31.768451 & 46.210182 & 31.789963 \\
\hline $\mathrm{H}$ & 45.504702 & 39.995690 & 36.644561 \\
\hline $\mathrm{H}$ & 41.457664 & 35.052319 & 33.770034 \\
\hline \multicolumn{4}{|c|}{ S1-CT2-MIN } \\
\hline C & 44.727617 & 39.903480 & 35.823057 \\
\hline C & 45.036479 & 39.242496 & 34.641507 \\
\hline $\mathrm{H}$ & 45.983488 & 38.746902 & 34.518387 \\
\hline C & 44.136489 & 39.208703 & 33.595747 \\
\hline $\mathrm{H}$ & 44.381848 & 38.708998 & 32.676884 \\
\hline C & 42.907762 & 39.853029 & 33.701324 \\
\hline 0 & 42.086042 & 39.821323 & 32.658757 \\
\hline $\mathrm{H}$ & 41.159680 & 40.055303 & 32.873007 \\
\hline C & 42.591585 & 40.512426 & 34.885092 \\
\hline $\mathrm{H}$ & 41.638150 & 41.000238 & 34.966678 \\
\hline C & 43.498792 & 40.539020 & 35.926083 \\
\hline $\mathrm{H}$ & 43.239619 & 41.074230 & 36.822903 \\
\hline C & 41.578430 & 35.978298 & 34.508274 \\
\hline $\mathrm{H}$ & 41.297078 & 35.641246 & 35.500470 \\
\hline $\mathrm{H}$ & 42.606280 & 36.324100 & 34.567892 \\
\hline
\end{tabular}




\begin{tabular}{|c|c|c|c|}
\hline C & 40.685965 & 37.147877 & 34.086595 \\
\hline $\mathrm{H}$ & 40.887019 & 37.986941 & 34.746261 \\
\hline $\mathrm{H}$ & 40.919078 & 37.515943 & 33.092525 \\
\hline C & 39.194437 & 36.906222 & 34.150284 \\
\hline 0 & 38.712507 & 35.851944 & 34.435179 \\
\hline 0 & 38.445904 & 37.938991 & 33.878996 \\
\hline $\mathrm{H}$ & 38.915051 & 38.775371 & 33.634247 \\
\hline $\mathrm{N}$ & 32.647281 & 46.767292 & 29.363630 \\
\hline $\mathrm{H}$ & 33.484078 & 46.241576 & 29.305418 \\
\hline C & 31.433071 & 46.064227 & 29.599982 \\
\hline C & 31.451503 & 44.627820 & 29.050237 \\
\hline$S$ & 32.461280 & 43.444072 & 30.047679 \\
\hline$C$ & 33.776399 & 42.858924 & 28.902220 \\
\hline$C$ & 35.111754 & 43.242667 & 29.322165 \\
\hline$C$ & 36.372669 & 42.516346 & 29.185013 \\
\hline$C$ & 36.996422 & 41.863432 & 30.273315 \\
\hline$C$ & 36.473784 & 41.411863 & 31.512195 \\
\hline$C$ & 37.324354 & 40.860979 & 32.450844 \\
\hline C & 38.732251 & 40.762481 & 32.256682 \\
\hline 0 & 39.500283 & 40.214442 & 33.154663 \\
\hline C & 39.236191 & 41.209172 & 31.002974 \\
\hline C & 38.389739 & 41.732139 & 30.046970 \\
\hline$C$ & 38.736473 & 42.256825 & 28.672572 \\
\hline C & 37.420752 & 42.884344 & 28.148966 \\
\hline $\mathrm{H}$ & 37.506929 & 43.966160 & 28.049990 \\
\hline $\mathrm{H}$ & 37.163121 & 42.498892 & 27.167043 \\
\hline $\mathrm{H}$ & 39.062731 & 41.450540 & 28.022116 \\
\hline $\mathrm{H}$ & 39.544445 & 42.980762 & 28.700504 \\
\hline $\mathrm{H}$ & 40.291631 & 41.120355 & 30.810690 \\
\hline $\mathrm{H}$ & 36.930089 & 40.505608 & 33.385847 \\
\hline $\mathrm{H}$ & 35.417139 & 41.447617 & 31.706775 \\
\hline $\mathrm{H}$ & 35.133949 & 44.201627 & 29.805575 \\
\hline 0 & 33.489953 & 42.191797 & 27.942932 \\
\hline $\mathrm{H}$ & 30.442479 & 44.247136 & 29.082965 \\
\hline $\mathrm{H}$ & 31.795196 & 44.617109 & 28.024594 \\
\hline $\mathrm{H}$ & 30.601908 & 46.551237 & 29.096497 \\
\hline C & 31.033213 & 46.069146 & 31.074772 \\
\hline 0 & 29.905008 & 45.933171 & 31.420539 \\
\hline $\mathrm{H}$ & 32.729093 & 47.731292 & 29.519700 \\
\hline $\mathrm{H}$ & 31.815588 & 46.229535 & 31.764872 \\
\hline $\mathrm{H}$ & 45.489420 & 40.045645 & 36.603511 \\
\hline $\mathrm{H}$ & 41.534230 & 35.107160 & 33.819160 \\
\hline
\end{tabular}

S1S0-1 


\begin{tabular}{|c|c|c|c|}
\hline C & 44.759886 & 39.871540 & 35.851312 \\
\hline C & 45.040547 & 39.123682 & 34.715952 \\
\hline $\mathrm{H}$ & 45.964848 & 38.582358 & 34.627664 \\
\hline C & 44.137973 & 39.060383 & 33.675628 \\
\hline $\mathrm{H}$ & 44.358268 & 38.486200 & 32.794983 \\
\hline C & 42.926655 & 39.745854 & 33.742743 \\
\hline 0 & 42.110482 & 39.691081 & 32.705056 \\
\hline $\mathrm{H}$ & 41.167283 & 39.930497 & 32.913294 \\
\hline C & 42.632270 & 40.475591 & 34.891938 \\
\hline $\mathrm{H}$ & 41.693840 & 40.994167 & 34.949161 \\
\hline C & 43.546761 & 40.541103 & 35.924067 \\
\hline $\mathrm{H}$ & 43.303851 & 41.136046 & 36.787907 \\
\hline C & 41.464068 & 35.916587 & 34.516655 \\
\hline $\mathrm{H}$ & 41.132856 & 35.537082 & 35.477397 \\
\hline $\mathrm{H}$ & 42.480432 & 36.278844 & 34.637836 \\
\hline C & 40.586640 & 37.092048 & 34.093658 \\
\hline $\mathrm{H}$ & 40.746289 & 37.912465 & 34.786168 \\
\hline $\mathrm{H}$ & 40.878041 & 37.497480 & 33.131577 \\
\hline C & 39.094766 & 36.849163 & 34.052006 \\
\hline 0 & 38.591749 & 35.778391 & 34.228529 \\
\hline 0 & 38.374416 & 37.906893 & 33.815557 \\
\hline $\mathrm{H}$ & 38.897952 & 38.733693 & 33.632169 \\
\hline $\mathrm{N}$ & 32.590243 & 46.872880 & 29.308083 \\
\hline $\mathrm{H}$ & 33.423173 & 46.359600 & 29.154513 \\
\hline C & 31.405517 & 46.138702 & 29.608002 \\
\hline C & 31.445026 & 44.736990 & 28.984708 \\
\hline S & 32.361859 & 43.384491 & 29.813931 \\
\hline C & 34.039292 & 43.550988 & 29.191692 \\
\hline C & 34.828478 & 42.290641 & 29.181790 \\
\hline C & 36.282037 & 42.238784 & 29.304710 \\
\hline C & 36.979028 & 41.802787 & 30.462914 \\
\hline C & 36.551027 & 41.498043 & 31.777081 \\
\hline C & 37.460562 & 40.971077 & 32.685355 \\
\hline C & 38.814943 & 40.712388 & 32.371202 \\
\hline 0 & 39.619463 & 40.102457 & 33.226097 \\
\hline C & 39.234243 & 41.071509 & 31.062379 \\
\hline C & 38.341847 & 41.593111 & 30.143299 \\
\hline C & 38.595187 & 41.992771 & 28.707695 \\
\hline C & 37.187627 & 42.153199 & 28.089081 \\
\hline $\mathrm{H}$ & 37.120821 & 43.032378 & 27.455945 \\
\hline $\mathrm{H}$ & 36.932179 & 41.293536 & 27.465475 \\
\hline $\mathrm{H}$ & 39.196947 & 41.269181 & 28.166951 \\
\hline $\mathrm{H}$ & 39.129687 & 42.938603 & 28.666208 \\
\hline $\mathrm{H}$ & 40.256005 & 40.881265 & 30.781143 \\
\hline
\end{tabular}




$\begin{array}{llll}\text { H } & 37.120510 & 40.696195 & 33.668921 \\ \text { H } & 35.527055 & 41.637343 & 32.080556 \\ \text { H } & 34.249155 & 41.412001 & 28.950982 \\ \text { O } & 34.484794 & 44.577885 & 28.778566 \\ \text { H } & 30.436610 & 44.348325 & 28.960236 \\ \text { H } & 31.803975 & 44.810444 & 27.967657 \\ \text { H } & 30.548913 & 46.615592 & 29.137475 \\ \text { C } & 31.008088 & 46.071800 & 31.087061 \\ \text { O } & 29.885344 & 45.882729 & 31.409133 \\ \text { H } & 32.683077 & 47.820433 & 29.534831 \\ \text { H } & 31.767945 & 46.235569 & 31.792656 \\ \text { H } & 45.513324 & 40.004066 & 36.641258 \\ \text { H } & 41.472210 & 35.073788 & 33.790549\end{array}$

$\begin{array}{llll}\text { S1S0-2 } & & & \\ \text { C } & 44.779627 & 39.870007 & 35.797167 \\ \text { C } & 45.097088 & 39.121275 & 34.672337 \\ \text { H } & 46.029078 & 38.590269 & 34.612213 \\ \text { C } & 44.224872 & 39.041016 & 33.607470 \\ \text { H } & 44.475202 & 38.462954 & 32.737182 \\ \text { C } & 43.005560 & 39.713902 & 33.636957 \\ \text { O } & 42.217497 & 39.638468 & 32.577639 \\ \text { H } & 41.270785 & 39.873201 & 32.754136 \\ \text { C } & 42.672711 & 40.447504 & 34.772745 \\ \text { H } & 41.728245 & 40.958015 & 34.800771 \\ \text { C } & 43.557660 & 40.527117 & 35.830483 \\ \text { H } & 43.284994 & 41.124893 & 36.683587 \\ \text { C } & 41.521714 & 35.928468 & 34.530259 \\ \text { H } & 41.205447 & 35.562541 & 35.500869 \\ \text { H } & 42.545807 & 36.276687 & 34.624759 \\ \text { C } & 40.649555 & 37.109090 & 34.113795 \\ \text { H } & 40.831965 & 37.938430 & 34.790808 \\ \text { H } & 40.915386 & 37.495763 & 33.137620 \\ \text { C } & 39.157457 & 36.870933 & 34.127864 \\ \text { O } & 38.659777 & 35.825972 & 34.430868 \\ \text { O } & 38.433510 & 37.901622 & 33.802950 \\ \text { H } & 38.955087 & 38.706638 & 33.537024 \\ \text { N } & 32.674161 & 46.683132 & 29.270387 \\ \text { H } & 33.502869 & 46.140540 & 29.285720 \\ \text { C } & 31.447402 & 46.010595 & 29.581355 \\ \text { C } & 31.467249 & 44.553202 & 29.098926 \\ \text { S } & 32.542463 & 43.462401 & 30.108670 \\ \text { C } & 33.813141 & 42.891768 & 28.933858 \\ \text { C } & 35.189445 & 43.231264 & 29.356374\end{array}$




$\begin{array}{llll}\text { C } & 36.424812 & 42.477605 & 29.170003 \\ \text { C } & 37.077403 & 41.820432 & 30.254271 \\ \text { C } & 36.609332 & 41.392961 & 31.517458 \\ \text { C } & 37.498617 & 40.822401 & 32.420697 \\ \text { C } & 38.877045 & 40.639586 & 32.148205 \\ \text { O } & 39.680215 & 40.040731 & 33.011328 \\ \text { C } & 39.325915 & 41.092950 & 30.880094 \\ \text { C } & 38.448609 & 41.654834 & 29.965577 \\ \text { C } & 38.748916 & 42.174637 & 28.574964 \\ \text { C } & 37.444852 & 42.873057 & 28.115458 \\ \text { H } & 37.567380 & 43.956688 & 28.065427 \\ \text { H } & 37.146204 & 42.549182 & 27.123602 \\ \text { H } & 38.993097 & 41.356891 & 27.902732 \\ \text { H } & 39.594181 & 42.855368 & 28.565475 \\ \text { H } & 40.368077 & 40.973937 & 30.635886 \\ \text { H } & 37.134348 & 40.480390 & 33.374003 \\ \text { H } & 35.572300 & 41.494727 & 31.790427 \\ \text { H } & 35.220847 & 44.195319 & 29.827875 \\ \text { O } & 33.543197 & 42.307223 & 27.931741 \\ \text { H } & 30.469694 & 44.146136 & 29.154857 \\ \text { H } & 31.795793 & 44.512863 & 28.069057 \\ \text { H } & 30.631944 & 46.478793 & 29.036806 \\ \text { C } & 31.014561 & 46.091152 & 31.054231 \\ \text { O } & 29.874046 & 46.016747 & 31.359514 \\ \text { H } & 32.778885 & 47.644090 & 29.431238 \\ \text { H } & 31.771459 & 46.237378 & 31.767696 \\ \text { H } & 45.512987 & 40.016689 & 36.603934 \\ \text { H } & 41.499108 & 35.080472 & 33.810430\end{array}$

The QM(CASSCF)/MM optimized minimum-energy isomerization path along the rotation of the $\mathrm{C} 10$ C11 bond in the $S_{1}$ state of the single-bond-rotation locked PYP chromophore in the R52A mutated protein.

$\begin{array}{lrrr}\text { C7-C10-C11-C12 0 } & & \\ \text { C } & 44.672911 & 39.887006 & 35.809531 \\ \text { C } & 44.931687 & 39.084310 & 34.706739 \\ \text { H } & 45.832864 & 38.501087 & 34.657015 \\ \text { C } & 44.047660 & 39.022761 & 33.648003 \\ \text { H } & 44.254613 & 38.408700 & 32.790590 \\ \text { C } & 42.880909 & 39.775683 & 33.667945 \\ \text { O } & 42.080251 & 39.710205 & 32.593909 \\ \text { H } & 41.255092 & 40.180773 & 32.698057 \\ \text { C } & 42.597399 & 40.560069 & 34.774414 \\ \text { H } & 41.693986 & 41.143168 & 34.794218\end{array}$




\begin{tabular}{|c|c|c|c|}
\hline C & 43.494942 & 40.616119 & 35.828402 \\
\hline $\mathrm{H}$ & 43.265770 & 41.256647 & 36.661694 \\
\hline C & 41.328725 & 35.911146 & 34.463952 \\
\hline $\mathrm{H}$ & 40.953955 & 35.544891 & 35.413021 \\
\hline $\mathrm{H}$ & 42.333196 & 36.288911 & 34.628749 \\
\hline C & 40.460280 & 37.073818 & 33.981252 \\
\hline $\mathrm{H}$ & 40.569698 & 37.901480 & 34.677399 \\
\hline $\mathrm{H}$ & 40.798601 & 37.467737 & 33.028235 \\
\hline C & 38.980295 & 36.800782 & 33.859471 \\
\hline 0 & 38.470560 & 35.743414 & 34.005772 \\
\hline 0 & 38.236577 & 37.859891 & 33.571898 \\
\hline $\mathrm{H}$ & 38.733379 & 38.659239 & 33.435942 \\
\hline $\mathrm{N}$ & 32.721528 & 46.604662 & 29.369464 \\
\hline $\mathrm{H}$ & 33.530585 & 46.032779 & 29.487270 \\
\hline C & 31.459386 & 45.977029 & 29.637776 \\
\hline C & 31.305201 & 44.631877 & 28.911643 \\
\hline$S$ & 32.040489 & 43.101444 & 29.591701 \\
\hline C & 33.819998 & 43.306532 & 29.442091 \\
\hline C & 34.506177 & 42.420948 & 28.499933 \\
\hline C & 35.683124 & 41.768924 & 28.731014 \\
\hline C & 36.551033 & 41.773815 & 29.936256 \\
\hline C & 36.210716 & 41.767236 & 31.309973 \\
\hline C & 37.171433 & 41.450905 & 32.248266 \\
\hline C & 38.525302 & 41.120350 & 31.876426 \\
\hline 0 & 39.361869 & 40.722005 & 32.713976 \\
\hline C & 38.861110 & 41.215725 & 30.472015 \\
\hline C & 37.881946 & 41.500080 & 29.547513 \\
\hline C & 37.961015 & 41.441192 & 28.037138 \\
\hline C & 36.511255 & 41.074401 & 27.666306 \\
\hline $\mathrm{H}$ & 36.233423 & 41.346053 & 26.654867 \\
\hline $\mathrm{H}$ & 36.402762 & 39.997337 & 27.766013 \\
\hline $\mathrm{H}$ & 38.690129 & 40.723899 & 27.676086 \\
\hline $\mathrm{H}$ & 38.221543 & 42.417827 & 27.632359 \\
\hline $\mathrm{H}$ & 39.864417 & 40.959169 & 30.179941 \\
\hline $\mathrm{H}$ & 36.922987 & 41.400152 & 33.292171 \\
\hline $\mathrm{H}$ & 35.207058 & 41.988257 & 31.612671 \\
\hline $\mathrm{H}$ & 34.012859 & 42.226619 & 27.560103 \\
\hline 0 & 34.430531 & 44.079348 & 30.203186 \\
\hline $\mathrm{H}$ & 30.250527 & 44.391715 & 28.879910 \\
\hline $\mathrm{H}$ & 31.650488 & 44.765630 & 27.895179 \\
\hline $\mathrm{H}$ & 30.675543 & 46.596687 & 29.202143 \\
\hline C & 31.062328 & 45.900559 & 31.116288 \\
\hline 0 & 29.926404 & 45.745924 & 31.435440 \\
\hline $\mathrm{H}$ & 32.832903 & 47.565624 & 29.529453 \\
\hline
\end{tabular}




$\begin{array}{llll}\mathrm{H} & 31.819382 & 46.062414 & 31.824898 \\ \mathrm{H} & 45.425354 & 40.021915 & 36.601163 \\ \mathrm{H} & 41.381186 & 35.058188 & 33.751009\end{array}$

$\begin{array}{llll}\text { C7-C10-C11-C12 10 } & & \\ \text { C } & 44.666357 & 39.886188 & 35.810117 \\ \text { C } & 44.921479 & 39.084122 & 34.706280 \\ \text { H } & 45.822782 & 38.501205 & 34.652818 \\ \text { C } & 44.033921 & 39.022752 & 33.650596 \\ \text { H } & 44.238681 & 38.409686 & 32.791880 \\ \text { C } & 42.866661 & 39.774591 & 33.675053 \\ \text { O } & 42.062879 & 39.709443 & 32.603706 \\ \text { H } & 41.231817 & 40.168103 & 32.717053 \\ \text { C } & 42.586544 & 40.558489 & 34.783233 \\ \text { H } & 41.682559 & 41.140600 & 34.806228 \\ \text { C } & 43.488014 & 40.614962 & 35.833826 \\ \text { H } & 43.262378 & 41.255588 & 36.668066 \\ \text { C } & 41.321558 & 35.908445 & 34.456665 \\ \text { H } & 40.946795 & 35.541596 & 35.405631 \\ \text { H } & 42.325221 & 36.287878 & 34.622091 \\ \text { C } & 40.451199 & 37.068985 & 33.972328 \\ \text { H } & 40.558207 & 37.897808 & 34.667209 \\ \text { H } & 40.789412 & 37.462283 & 33.019056 \\ \text { C } & 38.971715 & 36.794016 & 33.849385 \\ \text { O } & 38.464772 & 35.733876 & 33.988058 \\ \text { O } & 38.226458 & 37.853265 & 33.569823 \\ \text { H } & 38.720989 & 38.655747 & 33.439684 \\ \text { N } & 32.731373 & 46.593204 & 29.362428 \\ \text { H } & 33.540745 & 46.021657 & 29.477051 \\ \text { C } & 31.470712 & 45.963974 & 29.632826 \\ \text { C } & 31.315567 & 44.623964 & 28.897080 \\ \text { S } & 32.030654 & 43.080498 & 29.568346 \\ \text { C } & 33.818476 & 43.267268 & 29.446353 \\ \text { C } & 34.499095 & 42.365864 & 28.523379 \\ \text { C } & 35.731299 & 41.802515 & 28.727561 \\ \text { C } & 36.576904 & 41.778547 & 29.946764 \\ \text { C } & 36.215746 & 41.760055 & 31.315310 \\ \text { C } & 37.162031 & 41.427516 & 32.265231 \\ \text { C } & 38.518512 & 41.093053 & 31.912888 \\ \text { C } & 39.343301 & 40.685119 & 32.760058 \\ \text { C. } & 37.911685 & 41.493917 & 29.576764 \\ \text { C } & & 41.195388 & 30.514874 \\ \text { C } & 41.112085 & 27.666194\end{array}$




$\begin{array}{llll}\text { H } & 36.309887 & 41.404807 & 26.656082 \\ \mathrm{H} & 36.440521 & 40.034545 & 27.746387 \\ \mathrm{H} & 38.735754 & 40.730643 & 27.703679 \\ \mathrm{H} & 38.293242 & 42.430741 & 27.676856 \\ \mathrm{H} & 39.879902 & 40.931946 & 30.234251 \\ \mathrm{H} & 36.896395 & 41.366787 & 33.304349 \\ \mathrm{H} & 35.208857 & 41.981624 & 31.606305 \\ \mathrm{H} & 33.990986 & 42.131095 & 27.602069 \\ \mathrm{O} & 34.418699 & 44.053092 & 30.196777 \\ \mathrm{H} & 30.260111 & 44.389450 & 28.855939 \\ \mathrm{H} & 31.668277 & 44.762376 & 27.884009 \\ \mathrm{H} & 30.685850 & 46.585479 & 29.201933 \\ \mathrm{C} & 31.076543 & 45.880791 & 31.111533 \\ \mathrm{O} & 29.942549 & 45.715624 & 31.431324 \\ \mathrm{H} & 32.843766 & 47.553649 & 29.523210 \\ \mathrm{H} & 31.832351 & 46.050505 & 31.819364 \\ \mathrm{H} & 45.421850 & 40.019485 & 36.599048 \\ \mathrm{H} & 41.375836 & 35.055451 & 33.744018\end{array}$

$\begin{array}{llll}\text { C7-C10-C11-C12 20 } & & \\ \mathrm{C} & 44.663149 & 39.917622 & 35.816932 \\ \mathrm{C} & 44.909894 & 39.137526 & 34.695453 \\ \mathrm{H} & 45.814410 & 38.561367 & 34.621032 \\ \mathrm{C} & 44.008804 & 39.088677 & 33.650211 \\ \mathrm{H} & 44.205380 & 38.489059 & 32.780067 \\ \mathrm{C} & 42.834785 & 39.831323 & 33.699678 \\ \mathrm{O} & 42.019893 & 39.779863 & 32.640027 \\ \mathrm{H} & 41.150668 & 40.163967 & 32.786775 \\ \mathrm{C} & 42.567205 & 40.596874 & 34.824763 \\ \mathrm{H} & 41.659973 & 41.172375 & 34.866984 \\ \mathrm{C} & 43.480956 & 40.639776 & 35.865078 \\ \mathrm{H} & 43.261274 & 41.264508 & 36.712941 \\ \mathrm{C} & 41.353903 & 35.950809 & 34.475339 \\ \mathrm{H} & 40.989040 & 35.595355 & 35.432711 \\ \mathrm{H} & 42.362081 & 36.325727 & 34.624537 \\ \mathrm{C} & 40.483778 & 37.113138 & 33.990958 \\ \mathrm{H} & 40.616057 & 37.952399 & 34.668073 \\ \mathrm{H} & 40.802663 & 37.487773 & 33.023693 \\ \mathrm{C} & 38.998351 & 36.850407 & 33.907308 \\ \mathrm{O} & 38.495300 & 35.788302 & 34.065900 \\ \mathrm{O} & 38.250863 & 37.905489 & 33.644900 \\ \mathrm{H} & 38.730334 & 38.719899 & 33.492448 \\ \mathrm{~N} & 32.734366 & 46.645370 & 29.395486 \\ \mathrm{H} & 33.559155 & 46.096799 & 29.482296\end{array}$




\begin{tabular}{|c|c|c|c|}
\hline C & 31.488405 & 45.984295 & 29.657623 \\
\hline C & 31.388982 & 44.628791 & 28.940197 \\
\hline S & 32.056183 & 43.096964 & 29.676703 \\
\hline C & 33.872043 & 43.219457 & 29.541677 \\
\hline C & 34.490992 & 42.329142 & 28.628151 \\
\hline C & 35.855651 & 41.761768 & 28.790408 \\
\hline C & 36.615341 & 41.656147 & 29.966762 \\
\hline C & 36.227254 & 41.656939 & 31.345184 \\
\hline C & 37.149194 & 41.317309 & 32.316326 \\
\hline C & 38.505318 & 40.966414 & 32.015269 \\
\hline 0 & 39.311002 & 40.551564 & 32.889849 \\
\hline C & 38.899084 & 41.059970 & 30.625172 \\
\hline C & 37.977771 & 41.335776 & 29.654957 \\
\hline C & 38.138070 & 41.245035 & 28.155676 \\
\hline C & 36.819537 & 41.824703 & 27.615582 \\
\hline $\mathrm{H}$ & 36.959837 & 42.866146 & 27.328395 \\
\hline $\mathrm{H}$ & 36.453974 & 41.294832 & 26.739835 \\
\hline $\mathrm{H}$ & 38.235450 & 40.203231 & 27.862723 \\
\hline $\mathrm{H}$ & 39.015691 & 41.765923 & 27.786003 \\
\hline $\mathrm{H}$ & 39.916975 & 40.809491 & 30.377624 \\
\hline $\mathrm{H}$ & 36.850422 & 41.270765 & 33.348270 \\
\hline $\mathrm{H}$ & 35.215767 & 41.878354 & 31.620712 \\
\hline $\mathrm{H}$ & 33.822317 & 41.767863 & 27.998681 \\
\hline 0 & 34.460723 & 44.015515 & 30.278167 \\
\hline $\mathrm{H}$ & 30.339995 & 44.383685 & 28.842158 \\
\hline $\mathrm{H}$ & 31.795889 & 44.746200 & 27.945362 \\
\hline $\mathrm{H}$ & 30.694277 & 46.575395 & 29.203401 \\
\hline C & 31.075354 & 45.910768 & 31.132233 \\
\hline 0 & 29.939926 & 45.737487 & 31.437083 \\
\hline $\mathrm{H}$ & 32.828127 & 47.606659 & 29.562146 \\
\hline $\mathrm{H}$ & 31.821517 & 46.088743 & 31.848051 \\
\hline $\mathrm{H}$ & 45.425692 & 40.040670 & 36.601158 \\
\hline $\mathrm{H}$ & 41.394889 & 35.090156 & 33.770564 \\
\hline \multicolumn{4}{|c|}{$\mathrm{C} 7-\mathrm{C} 10-\mathrm{C} 11-\mathrm{C} 1230^{\circ}$} \\
\hline C & 44.669328 & 39.911567 & 35.817656 \\
\hline C & 44.920776 & 39.135768 & 34.694242 \\
\hline $\mathrm{H}$ & 45.828623 & 38.565053 & 34.617308 \\
\hline C & 44.018776 & 39.083672 & 33.649881 \\
\hline $\mathrm{H}$ & 44.219317 & 38.488233 & 32.777660 \\
\hline C & 42.838498 & 39.816706 & 33.702188 \\
\hline 0 & 42.021789 & 39.763024 & 32.646288 \\
\hline $\mathrm{H}$ & 41.143791 & 40.129933 & 32.806142 \\
\hline C & 42.566193 & 40.575821 & 34.831248 \\
\hline
\end{tabular}




\begin{tabular}{|c|c|c|c|}
\hline $\mathrm{H}$ & 41.653297 & 41.141589 & 34.876167 \\
\hline C & 43.481254 & 40.623373 & 35.869400 \\
\hline $\mathrm{H}$ & 43.258440 & 41.243752 & 36.719677 \\
\hline C & 41.343476 & 35.961154 & 34.470335 \\
\hline $\mathrm{H}$ & 40.983377 & 35.599448 & 35.427382 \\
\hline $\mathrm{H}$ & 42.351959 & 36.336179 & 34.617094 \\
\hline C & 40.471359 & 37.126193 & 33.998352 \\
\hline $\mathrm{H}$ & 40.607260 & 37.958431 & 34.683069 \\
\hline $\mathrm{H}$ & 40.786749 & 37.509646 & 33.033810 \\
\hline C & 38.984902 & 36.869967 & 33.919935 \\
\hline 0 & 38.478250 & 35.807013 & 34.068035 \\
\hline 0 & 38.245180 & 37.932420 & 33.674930 \\
\hline $\mathrm{H}$ & 38.733493 & 38.745298 & 33.522889 \\
\hline $\mathrm{N}$ & 32.708261 & 46.641817 & 29.382944 \\
\hline $\mathrm{H}$ & 33.544129 & 46.110364 & 29.467856 \\
\hline C & 31.481355 & 45.953132 & 29.667823 \\
\hline C & 31.418442 & 44.578532 & 28.979992 \\
\hline S & 32.112644 & 43.078603 & 29.761401 \\
\hline C & 33.924098 & 43.233942 & 29.554176 \\
\hline C & 34.545393 & 42.270254 & 28.712574 \\
\hline C & 35.944595 & 41.780872 & 28.880882 \\
\hline C & 36.679045 & 41.664155 & 30.063984 \\
\hline C & 36.266094 & 41.557212 & 31.431686 \\
\hline C & 37.181263 & 41.196860 & 32.397959 \\
\hline C & 38.562370 & 40.932659 & 32.106668 \\
\hline 0 & 39.361915 & 40.482411 & 32.976199 \\
\hline C & 38.982420 & 41.163810 & 30.745211 \\
\hline C & 38.073258 & 41.466661 & 29.773528 \\
\hline C & 38.287902 & 41.552482 & 28.281354 \\
\hline C & 36.931364 & 42.050344 & 27.750246 \\
\hline $\mathrm{H}$ & 36.964395 & 43.122423 & 27.550388 \\
\hline $\mathrm{H}$ & 36.646188 & 41.563640 & 26.821337 \\
\hline $\mathrm{H}$ & 38.511125 & 40.569028 & 27.876281 \\
\hline $\mathrm{H}$ & 39.115576 & 42.201669 & 28.007611 \\
\hline $\mathrm{H}$ & 40.019629 & 40.999545 & 30.505904 \\
\hline $\mathrm{H}$ & 36.863786 & 41.068720 & 33.417416 \\
\hline $\mathrm{H}$ & 35.238796 & 41.716057 & 31.700383 \\
\hline $\mathrm{H}$ & 33.859397 & 41.570958 & 28.265126 \\
\hline 0 & 34.504731 & 44.115573 & 30.169927 \\
\hline $\mathrm{H}$ & 30.376285 & 44.307968 & 28.875583 \\
\hline $\mathrm{H}$ & 31.835353 & 44.678460 & 27.987155 \\
\hline $\mathrm{H}$ & 30.667459 & 46.511020 & 29.207523 \\
\hline C & 31.080815 & 45.892273 & 31.145717 \\
\hline 0 & 29.951170 & 45.700599 & 31.460208 \\
\hline
\end{tabular}




$\begin{array}{llll}\mathrm{H} & 32.787238 & 47.603870 & 29.553111 \\ \mathrm{H} & 31.828885 & 46.091062 & 31.853558 \\ \mathrm{H} & 45.430487 & 40.037752 & 36.602569 \\ \mathrm{H} & 41.381797 & 35.104617 & 33.760449\end{array}$

\begin{tabular}{|c|c|c|c|}
\hline \multicolumn{4}{|c|}{ C7-C10-C11-C12 40 } \\
\hline C & 44.701597 & 39.916638 & 35.809896 \\
\hline C & 44.979178 & 39.156372 & 34.682423 \\
\hline $\mathrm{H}$ & 45.893620 & 38.595780 & 34.615132 \\
\hline C & 44.096067 & 39.105675 & 33.622225 \\
\hline $\mathrm{H}$ & 44.317593 & 38.521057 & 32.747694 \\
\hline C & 42.906414 & 39.825398 & 33.660515 \\
\hline 0 & 42.111245 & 39.777107 & 32.591651 \\
\hline $\mathrm{H}$ & 41.215675 & 40.121251 & 32.739549 \\
\hline C & 42.607161 & 40.568363 & 34.795211 \\
\hline $\mathrm{H}$ & 41.686282 & 41.121707 & 34.830225 \\
\hline C & 43.504109 & 40.614716 & 35.848996 \\
\hline $\mathrm{H}$ & 43.259644 & 41.223082 & 36.702340 \\
\hline C & 41.401837 & 35.984392 & 34.468602 \\
\hline $\mathrm{H}$ & 41.043407 & 35.640807 & 35.433082 \\
\hline $\mathrm{H}$ & 42.420965 & 36.336056 & 34.600443 \\
\hline C & 40.553185 & 37.164671 & 33.992850 \\
\hline $\mathrm{H}$ & 40.725568 & 38.006921 & 34.656331 \\
\hline $\mathrm{H}$ & 40.857526 & 37.522235 & 33.014837 \\
\hline C & 39.059140 & 36.945357 & 33.951411 \\
\hline 0 & 38.532770 & 35.897732 & 34.145221 \\
\hline 0 & 38.341607 & 38.015462 & 33.690404 \\
\hline $\mathrm{H}$ & 38.844108 & 38.818337 & 33.503852 \\
\hline N & 32.699095 & 46.700319 & 29.407808 \\
\hline $\mathrm{H}$ & 33.545921 & 46.180616 & 29.419772 \\
\hline C & 31.495549 & 45.980986 & 29.698667 \\
\hline C & 31.487107 & 44.579270 & 29.069392 \\
\hline$S$ & 32.325600 & 43.163896 & 29.873209 \\
\hline C & 34.055583 & 43.306079 & 29.320755 \\
\hline C & 34.597725 & 42.125379 & 28.692089 \\
\hline C & 35.978182 & 41.613674 & 28.862827 \\
\hline C & 36.755477 & 41.558900 & 30.030682 \\
\hline C & 36.375148 & 41.485583 & 31.403824 \\
\hline C & 37.313529 & 41.161283 & 32.359652 \\
\hline C & 38.687579 & 40.888220 & 32.044312 \\
\hline 0 & 39.501783 & 40.456769 & 32.924442 \\
\hline C & 39.072147 & 41.084236 & 30.676779 \\
\hline C & 38.136331 & 41.357609 & 29.716429 \\
\hline C & 38.316165 & 41.404143 & 28.217399 \\
\hline
\end{tabular}




\begin{tabular}{|c|c|c|c|}
\hline C & 36.944235 & 41.876448 & 27.707628 \\
\hline $\mathrm{H}$ & 36.960601 & 42.945834 & 27.499093 \\
\hline $\mathrm{H}$ & 36.642005 & 41.374001 & 26.793198 \\
\hline $\mathrm{H}$ & 38.534057 & 40.413154 & 27.832289 \\
\hline $\mathrm{H}$ & 39.129737 & 42.053149 & 27.909889 \\
\hline $\mathrm{H}$ & 40.103614 & 40.918724 & 30.414411 \\
\hline $\mathrm{H}$ & 37.019852 & 41.067409 & 33.390075 \\
\hline $\mathrm{H}$ & 35.352453 & 41.651240 & 31.693588 \\
\hline $\mathrm{H}$ & 33.849540 & 41.354812 & 28.602888 \\
\hline 0 & 34.656218 & 44.339383 & 29.510629 \\
\hline $\mathrm{H}$ & 30.461796 & 44.237680 & 29.037086 \\
\hline $\mathrm{H}$ & 31.844713 & 44.653043 & 28.051933 \\
\hline $\mathrm{H}$ & 30.660752 & 46.488414 & 29.217342 \\
\hline C & 31.089824 & 45.935052 & 31.174839 \\
\hline 0 & 29.962427 & 45.733926 & 31.490111 \\
\hline $\mathrm{H}$ & 32.768556 & 47.660232 & 29.592575 \\
\hline $\mathrm{H}$ & 31.838057 & 46.135455 & 31.881380 \\
\hline $\mathrm{H}$ & 45.450769 & 40.045437 & 36.605917 \\
\hline $\mathrm{H}$ & 41.412022 & 35.120397 & 33.766787 \\
\hline \multicolumn{4}{|c|}{$\mathrm{C} 7-\mathrm{C} 10-\mathrm{C} 11-\mathrm{C} 1250^{\circ}$} \\
\hline C & 44.703627 & 39.931043 & 35.825220 \\
\hline C & 44.967104 & 39.179718 & 34.688256 \\
\hline $\mathrm{H}$ & 45.880424 & 38.618790 & 34.604267 \\
\hline C & 44.069957 & 39.138334 & 33.639546 \\
\hline $\mathrm{H}$ & 44.280657 & 38.561079 & 32.757297 \\
\hline C & 42.879247 & 39.856535 & 33.698304 \\
\hline 0 & 42.070032 & 39.816564 & 32.643091 \\
\hline $\mathrm{H}$ & 41.163739 & 40.139436 & 32.814562 \\
\hline C & 42.595129 & 40.589012 & 34.844750 \\
\hline $\mathrm{H}$ & 41.673459 & 41.139245 & 34.896523 \\
\hline C & 43.506209 & 40.627278 & 35.885916 \\
\hline $\mathrm{H}$ & 43.273337 & 41.227733 & 36.748110 \\
\hline C & 41.414612 & 36.005768 & 34.480155 \\
\hline $\mathrm{H}$ & 41.062425 & 35.651023 & 35.443008 \\
\hline $\mathrm{H}$ & 42.433455 & 36.359042 & 34.609575 \\
\hline C & 40.560918 & 37.189502 & 34.023010 \\
\hline $\mathrm{H}$ & 40.736910 & 38.020747 & 34.699030 \\
\hline $\mathrm{H}$ & 40.860271 & 37.560846 & 33.049017 \\
\hline C & 39.066164 & 36.974330 & 33.989131 \\
\hline 0 & 38.539599 & 35.923453 & 34.174386 \\
\hline 0 & 38.354390 & 38.048533 & 33.743968 \\
\hline $\mathrm{H}$ & 38.861375 & 38.856463 & 33.559807 \\
\hline $\mathrm{N}$ & 32.646025 & 46.729777 & 29.43354 \\
\hline
\end{tabular}




\begin{tabular}{|c|c|c|c|}
\hline $\mathrm{H}$ & 33.495350 & 46.216255 & 29.393851 \\
\hline C & 31.457318 & 45.992916 & 29.739002 \\
\hline C & 31.481941 & 44.586607 & 29.121853 \\
\hline$S$ & 32.376004 & 43.207143 & 29.933262 \\
\hline C & 34.065158 & 43.347679 & 29.275138 \\
\hline C & 34.629248 & 42.104381 & 28.774886 \\
\hline C & 36.021045 & 41.656588 & 28.957925 \\
\hline C & 36.778879 & 41.591346 & 30.148163 \\
\hline C & 36.378093 & 41.435976 & 31.504693 \\
\hline C & 37.312183 & 41.100563 & 32.462837 \\
\hline C & 38.696941 & 40.891570 & 32.165950 \\
\hline 0 & 39.509117 & 40.431823 & 33.048768 \\
\hline C & 39.095672 & 41.166524 & 30.823137 \\
\hline C & 38.167644 & 41.462610 & 29.855785 \\
\hline C & 38.388945 & 41.598886 & 28.368613 \\
\hline C & 37.001137 & 41.988532 & 27.831785 \\
\hline $\mathrm{H}$ & 36.953451 & 43.057039 & 27.623563 \\
\hline $\mathrm{H}$ & 36.752921 & 41.472467 & 26.908475 \\
\hline $\mathrm{H}$ & 38.716522 & 40.657496 & 27.937958 \\
\hline $\mathrm{H}$ & 39.151073 & 42.333691 & 28.123823 \\
\hline $\mathrm{H}$ & 40.134240 & 41.044471 & 30.565496 \\
\hline $\mathrm{H}$ & 36.996763 & 40.937441 & 33.478142 \\
\hline $\mathrm{H}$ & 35.344863 & 41.542575 & 31.785948 \\
\hline $\mathrm{H}$ & 33.895438 & 41.315543 & 28.786587 \\
\hline 0 & 34.624771 & 44.416102 & 29.263358 \\
\hline $\mathrm{H}$ & 30.467599 & 44.213655 & 29.100078 \\
\hline $\mathrm{H}$ & 31.831853 & 44.660618 & 28.101851 \\
\hline $\mathrm{H}$ & 30.607776 & 46.476146 & 29.258807 \\
\hline C & 31.061395 & 45.946919 & 31.217459 \\
\hline 0 & 29.938193 & 45.734388 & 31.538830 \\
\hline $\mathrm{H}$ & 32.717177 & 47.685382 & 29.637993 \\
\hline $\mathrm{H}$ & 31.811999 & 46.154178 & 31.919340 \\
\hline $\mathrm{H}$ & 45.461667 & 40.052580 & 36.613780 \\
\hline $\mathrm{H}$ & 41.422506 & 35.149199 & 33.7693 \\
\hline \multicolumn{4}{|c|}{ C7-C10-C11-C12 60 } \\
\hline$C$ & 44.699547 & 39.920710 & 35.825260 \\
\hline C & 44.969241 & 39.172442 & 34.688319 \\
\hline $\mathrm{H}$ & 45.886793 & 38.618728 & 34.605254 \\
\hline C & 44.072668 & 39.123347 & 33.639193 \\
\hline $\mathrm{H}$ & 44.287461 & 38.546286 & 32.757851 \\
\hline C & 42.876165 & 39.831867 & $33.69765 c$ \\
\hline 0 & 42.066140 & 39.787833 & 32.64370 \\
\hline $\mathrm{H}$ & 41.151988 & 40.091720 & 32.8227 \\
\hline
\end{tabular}




\begin{tabular}{|c|c|c|c|}
\hline C & 42.587111 & 40.564428 & 34.843356 \\
\hline $\mathrm{H}$ & 41.660880 & 41.107140 & 34.894392 \\
\hline C & 43.497510 & 40.610159 & 35.884478 \\
\hline $\mathrm{H}$ & 43.260059 & 41.210052 & 36.745961 \\
\hline C & 41.387830 & 35.977692 & 34.481903 \\
\hline $\mathrm{H}$ & 41.026787 & 35.618306 & 35.439860 \\
\hline $\mathrm{H}$ & 42.406688 & 36.327160 & 34.623318 \\
\hline C & 40.541760 & 37.164569 & 34.021379 \\
\hline $\mathrm{H}$ & 40.706722 & 37.995191 & 34.700776 \\
\hline $\mathrm{H}$ & 40.855870 & 37.540870 & 33.054091 \\
\hline C & 39.047473 & 36.948632 & 33.960258 \\
\hline 0 & 38.520875 & 35.894275 & 34.131592 \\
\hline 0 & 38.339952 & 38.022104 & 33.711352 \\
\hline $\mathrm{H}$ & 38.850097 & 38.837249 & 33.543060 \\
\hline$N$ & 32.689977 & 46.771958 & 29.363343 \\
\hline $\mathrm{H}$ & 33.535660 & 46.257828 & 29.285847 \\
\hline C & 31.501737 & 46.035507 & 29.663287 \\
\hline C & 31.528604 & 44.632693 & 29.040988 \\
\hline$S$ & 32.451237 & 43.264597 & 29.840081 \\
\hline C & 34.127006 & 43.403185 & 29.159819 \\
\hline C & 34.722633 & 42.131786 & 28.756968 \\
\hline C & 36.128898 & 41.743006 & 28.934250 \\
\hline C & 36.853689 & 41.632218 & 30.141374 \\
\hline C & 36.416333 & 41.420127 & 31.477469 \\
\hline C & 37.327138 & 41.053311 & 32.447793 \\
\hline C & 38.718017 & 40.859798 & 32.178544 \\
\hline 0 & 39.513911 & 40.369347 & 33.068958 \\
\hline C & 39.152409 & 41.176881 & 30.858574 \\
\hline C & 38.247319 & 41.516812 & 29.879488 \\
\hline C & 38.503652 & 41.695221 & 28.400547 \\
\hline C & 37.125561 & 42.081931 & 27.829835 \\
\hline $\mathrm{H}$ & 37.082665 & 43.149211 & 27.618987 \\
\hline $\mathrm{H}$ & 36.900449 & 41.560413 & 26.903260 \\
\hline $\mathrm{H}$ & 38.851789 & 40.766365 & 27.959305 \\
\hline $\mathrm{H}$ & 39.260279 & 42.445949 & 28.195971 \\
\hline $\mathrm{H}$ & 40.197045 & 41.061249 & 30.623505 \\
\hline $\mathrm{H}$ & 36.986709 & 40.850436 & 33.447787 \\
\hline $\mathrm{H}$ & 35.375025 & 41.512088 & 31.733126 \\
\hline $\mathrm{H}$ & 34.004124 & 41.329941 & 28.742656 \\
\hline 0 & 34.659201 & 44.477423 & 29.040489 \\
\hline $\mathrm{H}$ & 30.517633 & 44.249460 & 29.036819 \\
\hline $\mathrm{H}$ & 31.861838 & 44.709708 & 28.015648 \\
\hline $\mathrm{H}$ & 30.650945 & 46.518655 & 29.184829 \\
\hline C & 31.103652 & 45.978162 & 31.142698 \\
\hline
\end{tabular}




$\begin{array}{llll}\mathrm{O} & 29.979441 & 45.766270 & 31.460717 \\ \mathrm{H} & 32.766636 & 47.725825 & 29.572324 \\ \mathrm{H} & 31.853222 & 46.174414 & 31.848809 \\ \mathrm{H} & 45.454889 & 40.045699 & 36.615510 \\ \mathrm{H} & 41.400782 & 35.124717 & 33.767066\end{array}$

$\begin{array}{llll}\text { C7-C10-C11-C12 70 } & & \\ \mathrm{C} & 44.714061 & 39.909181 & 35.834217 \\ \mathrm{C} & 44.982849 & 39.160249 & 34.697315 \\ \mathrm{H} & 45.899937 & 38.605708 & 34.613748 \\ \mathrm{C} & 44.085737 & 39.111470 & 33.648721 \\ \mathrm{H} & 44.299851 & 38.534042 & 32.767385 \\ \mathrm{C} & 42.889408 & 39.820348 & 33.707346 \\ \mathrm{O} & 42.078824 & 39.777104 & 32.654331 \\ \mathrm{H} & 41.161862 & 40.073246 & 32.838616 \\ \mathrm{C} & 42.601250 & 40.552530 & 34.853683 \\ \mathrm{H} & 41.675081 & 41.094996 & 34.904742 \\ \mathrm{C} & 43.511893 & 40.597790 & 35.894405 \\ \mathrm{H} & 43.274937 & 41.196624 & 36.756658 \\ \mathrm{C} & 41.398724 & 35.983195 & 34.482021 \\ \mathrm{H} & 41.044295 & 35.618042 & 35.440277 \\ \mathrm{H} & 42.416127 & 36.337949 & 34.619806 \\ \mathrm{C} & 40.544087 & 37.165906 & 34.029756 \\ \mathrm{H} & 40.707314 & 37.993768 & 34.712665 \\ \mathrm{H} & 40.852279 & 37.547740 & 33.063109 \\ \mathrm{C} & 39.050907 & 36.943320 & 33.974736 \\ \mathrm{O} & 38.529207 & 35.886248 & 34.145450 \\ \mathrm{O} & 38.341192 & 38.015528 & 33.730544 \\ \mathrm{H} & 38.854783 & 38.829993 & 33.560239 \\ \mathrm{~N} & 32.670771 & 46.792383 & 29.361766 \\ \mathrm{H} & 33.510884 & 46.272833 & 29.265387 \\ \mathrm{C} & 31.479297 & 46.060885 & 29.661146 \\ \mathrm{C} & 31.507320 & 44.658199 & 29.038608 \\ \mathrm{C} & 32.418180 & 43.290114 & 29.850486 \\ \mathrm{C} & 34.099800 & 43.418767 & 29.187043 \\ \mathrm{C} & 34.720429 & 42.138501 & 28.858370 \\ \mathrm{C} & 36.145096 & 41.825420 & 29.001266 \\ \mathrm{C} & 36.867739 & 41.660846 & 30.200898 \\ \mathrm{C} & 36.433660 & 41.418351 & 31.531903 \\ \mathrm{C} & 37.347291 & 41.028353 & 32.490696 \\ \mathrm{C} & 38.735815 & 40.843500 & 32.215346 \\ \mathrm{C} & 39.535109 & 40.336045 & 33.097053 \\ \mathrm{C} & 38.260593 & 41.551284 & 29.932187\end{array}$




\begin{tabular}{|c|c|c|c|}
\hline C & 38.513585 & 41.769193 & 28.457128 \\
\hline C & 37.128546 & 42.132754 & 27.881188 \\
\hline $\mathrm{H}$ & 37.081301 & 43.190866 & 27.629165 \\
\hline $\mathrm{H}$ & 36.900001 & 41.577942 & 26.973871 \\
\hline $\mathrm{H}$ & 38.896708 & 40.863183 & 27.995379 \\
\hline $\mathrm{H}$ & 39.245183 & 42.550950 & 28.273927 \\
\hline $\mathrm{H}$ & 40.209575 & 41.063932 & 30.657805 \\
\hline $\mathrm{H}$ & 37.006787 & 40.797789 & 33.484677 \\
\hline $\mathrm{H}$ & 35.393239 & 41.502605 & 31.792956 \\
\hline $\mathrm{H}$ & 34.024220 & 41.321230 & 28.796410 \\
\hline 0 & 34.621656 & 44.489722 & 29.009540 \\
\hline $\mathrm{H}$ & 30.495434 & 44.278047 & 29.021256 \\
\hline $\mathrm{H}$ & 31.854471 & 44.733235 & 28.017824 \\
\hline $\mathrm{H}$ & 30.629846 & 46.546216 & 29.182848 \\
\hline C & 31.082557 & 46.003111 & 31.140574 \\
\hline 0 & 29.956372 & 45.802866 & 31.459008 \\
\hline $\mathrm{H}$ & 32.758184 & 47.742471 & 29.582632 \\
\hline $\mathrm{H}$ & 31.835241 & 46.188620 & 31.846189 \\
\hline $\mathrm{H}$ & 45.469692 & 40.033079 & 36.624222 \\
\hline $\mathrm{H}$ & 41.412390 & 35.133227 & 33.764007 \\
\hline \multicolumn{4}{|c|}{$\mathrm{C} 7-\mathrm{C} 10-\mathrm{C} 11-\mathrm{C} 1280^{\circ}$} \\
\hline C & 44.734235 & 39.891872 & 35.836353 \\
\hline C & 45.008474 & 39.138854 & 34.703708 \\
\hline $\mathrm{H}$ & 45.927206 & 38.586779 & 34.625067 \\
\hline C & 44.113874 & 39.081434 & 33.653386 \\
\hline $\mathrm{H}$ & 44.331930 & 38.500349 & 32.775422 \\
\hline C & 42.914177 & 39.784725 & 33.706972 \\
\hline 0 & 42.104705 & 39.732521 & 32.653881 \\
\hline $\mathrm{H}$ & 41.184806 & 40.021716 & 32.839089 \\
\hline C & 42.620700 & 40.521473 & 34.849244 \\
\hline $\mathrm{H}$ & 41.691455 & 41.058974 & 34.896285 \\
\hline C & 43.529116 & 40.575889 & 35.891160 \\
\hline $\mathrm{H}$ & 43.287839 & 41.177523 & 36.750330 \\
\hline C & 41.414170 & 35.952683 & 34.482294 \\
\hline $\mathrm{H}$ & 41.063495 & 35.585070 & 35.440929 \\
\hline $\mathrm{H}$ & 42.431202 & 36.309507 & 34.617470 \\
\hline C & 40.554892 & 37.133320 & 34.034996 \\
\hline $\mathrm{H}$ & 40.716587 & 37.961166 & 34.718268 \\
\hline $\mathrm{H}$ & 40.858909 & 37.518211 & 33.068274 \\
\hline C & 39.062283 & 36.905686 & 33.982038 \\
\hline 0 & 38.543091 & 35.846940 & 34.151006 \\
\hline 0 & 38.350734 & 37.976849 & 33.739984 \\
\hline $\mathrm{H}$ & 38.867037 & 38.790580 & 33.570493 \\
\hline
\end{tabular}




\begin{tabular}{|c|c|c|c|}
\hline$N$ & 32.646299 & 46.772301 & 29.330837 \\
\hline $\mathrm{H}$ & 33.485523 & 46.251034 & 29.235815 \\
\hline C & 31.454199 & 46.044138 & 29.634229 \\
\hline C & 31.481339 & 44.640128 & 29.014368 \\
\hline$S$ & 32.391496 & 43.276691 & 29.832177 \\
\hline C & 34.081055 & 43.414209 & 29.197415 \\
\hline C & 34.740032 & 42.136757 & 28.946837 \\
\hline C & 36.178368 & 41.903437 & 29.066588 \\
\hline C & 36.899841 & 41.677778 & 30.251698 \\
\hline C & 36.466649 & 41.407298 & 31.577661 \\
\hline C & 37.380701 & 40.990397 & 32.524309 \\
\hline C & 38.766339 & 40.804080 & 32.239734 \\
\hline 0 & 39.567157 & 40.277367 & 33.110099 \\
\hline C & 39.196678 & 41.160839 & 30.928673 \\
\hline C & 38.290876 & 41.562651 & 29.973616 \\
\hline C & 38.541769 & 41.827501 & 28.505082 \\
\hline C & 37.146631 & 42.156172 & 27.924395 \\
\hline $\mathrm{H}$ & 37.092182 & 43.197367 & 27.614453 \\
\hline $\mathrm{H}$ & 36.911116 & 41.550779 & 27.051127 \\
\hline $\mathrm{H}$ & 38.970551 & 40.954294 & 28.021371 \\
\hline $\mathrm{H}$ & 39.238257 & 42.647188 & 28.353190 \\
\hline $\mathrm{H}$ & 40.237958 & 41.039559 & 30.682000 \\
\hline $\mathrm{H}$ & 37.042601 & 40.739801 & 33.514151 \\
\hline $\mathrm{H}$ & 35.427420 & 41.493552 & 31.842604 \\
\hline $\mathrm{H}$ & 34.075224 & 41.300016 & 28.830121 \\
\hline 0 & 34.586289 & 44.485591 & 28.979250 \\
\hline $\mathrm{H}$ & 30.469698 & 44.259425 & 28.996801 \\
\hline $\mathrm{H}$ & 31.830231 & 44.713277 & 27.994056 \\
\hline $\mathrm{H}$ & 30.604444 & 46.529676 & 29.156641 \\
\hline C & 31.059503 & 45.989976 & 31.114851 \\
\hline 0 & 29.934203 & 45.788536 & 31.435456 \\
\hline $\mathrm{H}$ & 32.734132 & 47.724251 & 29.542349 \\
\hline $\mathrm{H}$ & 31.812564 & 46.179414 & 31.819225 \\
\hline $\mathrm{H}$ & 45.486084 & 40.019897 & 36.629216 \\
\hline $\mathrm{H}$ & 41.427297 & 35.104548 & 33.762236 \\
\hline \multicolumn{4}{|c|}{ C7-C10-C11-C12 90 } \\
\hline C & 44.733623 & 39.870411 & 35.816137 \\
\hline C & 45.026350 & 39.121727 & 34.685421 \\
\hline $\mathrm{H}$ & 45.952883 & 38.582249 & 34.613693 \\
\hline C & 44.139848 & 39.051407 & 33.629089 \\
\hline $\mathrm{H}$ & 44.371049 & 38.472453 & 32.753134 \\
\hline C & 42.930603 & 39.738016 & 33.675383 \\
\hline 0 & 42.125410 & 39.671463 & 32.619543 \\
\hline
\end{tabular}




\begin{tabular}{|c|c|c|c|}
\hline $\mathrm{H}$ & 41.204969 & 39.956740 & 32.803115 \\
\hline C & 42.620400 & 40.473353 & 34.813808 \\
\hline $\mathrm{H}$ & 41.683389 & 40.997510 & 34.853522 \\
\hline C & 43.520068 & 40.539728 & 35.862171 \\
\hline $\mathrm{H}$ & 43.264882 & 41.138227 & 36.719472 \\
\hline C & 41.421807 & 35.906998 & 34.516394 \\
\hline $\mathrm{H}$ & 41.070357 & 35.540965 & 35.474988 \\
\hline $\mathrm{H}$ & 42.439592 & 36.262051 & 34.651342 \\
\hline C & 40.566463 & 37.089803 & 34.067230 \\
\hline $\mathrm{H}$ & 40.724749 & 37.915359 & 34.754454 \\
\hline $\mathrm{H}$ & 40.878671 & 37.476637 & 33.103985 \\
\hline C & 39.074164 & 36.862397 & 34.003569 \\
\hline 0 & 38.554200 & 35.806109 & 34.185328 \\
\hline 0 & 38.364514 & 37.928683 & 33.737811 \\
\hline $\mathrm{H}$ & 38.882129 & 38.739985 & 33.557773 \\
\hline $\mathrm{N}$ & 32.602754 & 46.830573 & 29.355725 \\
\hline $\mathrm{H}$ & 33.444442 & 46.314089 & 29.258009 \\
\hline C & 31.414548 & 46.092253 & 29.650767 \\
\hline C & 31.448201 & 44.693553 & 29.018309 \\
\hline$S$ & 32.360247 & 43.325636 & 29.823409 \\
\hline C & 34.055205 & 43.484471 & 29.214925 \\
\hline C & 34.751187 & 42.215306 & 29.040091 \\
\hline C & 36.197877 & 42.046951 & 29.137133 \\
\hline C & 36.923104 & 41.744633 & 30.299874 \\
\hline C & 36.496864 & 41.449890 & 31.623564 \\
\hline C & 37.410492 & 40.983381 & 32.547109 \\
\hline C & 38.787334 & 40.769822 & 32.240688 \\
\hline 0 & 39.587016 & 40.209852 & 33.090642 \\
\hline C & 39.209835 & 41.137963 & 30.930014 \\
\hline C & 38.306053 & 41.593390 & 29.998995 \\
\hline C & 38.548319 & 41.907783 & 28.539661 \\
\hline C & 37.141374 & 42.195091 & 27.959702 \\
\hline $\mathrm{H}$ & 37.085763 & 43.200797 & 27.551673 \\
\hline $\mathrm{H}$ & 36.884567 & 41.510398 & 27.152105 \\
\hline $\mathrm{H}$ & 39.027409 & 41.076538 & 28.030693 \\
\hline $\mathrm{H}$ & 39.200317 & 42.768928 & 28.422112 \\
\hline $\mathrm{H}$ & 40.243031 & 40.987345 & 30.666629 \\
\hline $\mathrm{H}$ & 37.078017 & 40.714652 & 33.534217 \\
\hline $\mathrm{H}$ & 35.463421 & 41.556767 & 31.902720 \\
\hline $\mathrm{H}$ & 34.116636 & 41.360815 & 28.888742 \\
\hline 0 & 34.536728 & 44.558089 & 28.958475 \\
\hline $\mathrm{H}$ & 30.437163 & 44.310915 & 28.997892 \\
\hline $\mathrm{H}$ & 31.796296 & 44.776906 & 27.998588 \\
\hline $\mathrm{H}$ & 30.563186 & 46.577026 & 29.175468 \\
\hline
\end{tabular}




$\begin{array}{llll}\mathrm{C} & 31.016598 & 46.022776 & 31.130073 \\ \mathrm{O} & 29.891139 & 45.815218 & 31.445733 \\ \mathrm{H} & 32.686121 & 47.779280 & 29.583374 \\ \mathrm{H} & 31.768096 & 46.206079 & 31.838082 \\ \mathrm{H} & 45.476804 & 40.006837 & 36.615506 \\ \mathrm{H} & 41.433649 & 35.058270 & 33.796499\end{array}$

$\begin{array}{llll}\text { C7-C10-C11-C12 100 } & & \\ \text { C } & 44.760173 & 39.854258 & 35.841489 \\ \text { C } & 45.046825 & 39.094633 & 34.716792 \\ \text { H } & 45.970032 & 38.549520 & 34.647643 \\ \text { C } & 44.157601 & 39.019253 & 33.663054 \\ \text { H } & 44.384571 & 38.432224 & 32.791337 \\ \text { C } & 42.950717 & 39.709794 & 33.707052 \\ \text { O } & 42.142924 & 39.639451 & 32.653739 \\ \text { H } & 41.219782 & 39.915473 & 32.844304 \\ \text { C } & 42.645021 & 40.453771 & 34.841577 \\ \text { H } & 41.709233 & 40.980144 & 34.880456 \\ \text { C } & 43.548132 & 40.526573 & 35.886269 \\ \text { H } & 43.296918 & 41.131854 & 36.740069 \\ \text { C } & 41.434152 & 35.899126 & 34.494465 \\ \text { H } & 41.093283 & 35.522696 & 35.453026 \\ \text { H } & 42.448993 & 36.263795 & 34.624861 \\ \text { C } & 40.561013 & 37.072461 & 34.059762 \\ \text { H } & 40.712368 & 37.896304 & 34.750193 \\ \text { H } & 40.859706 & 37.469223 & 33.096555 \\ \text { C } & 39.071403 & 36.827147 & 34.004072 \\ \text { O } & 38.562583 & 35.763051 & 34.170473 \\ \text { O } & 38.351328 & 37.892188 & 33.761178 \\ \text { H } & 38.871165 & 38.704853 & 33.594787 \\ \text { N } & 32.600277 & 46.811467 & 29.311579 \\ \text { H } & 33.433397 & 46.289395 & 29.179127 \\ \text { C } & 31.407567 & 46.082432 & 29.609436 \\ \text { C } & 31.441062 & 44.680980 & 28.983033 \\ \text { S } & 32.352955 & 43.324917 & 29.806743 \\ \text { C } & 34.049828 & 43.481194 & 29.209827 \\ \text { C } & 34.779502 & 42.219858 & 29.144094 \\ \text { C } & 36.231608 & 42.115480 & 29.241489 \\ \text { C } & 36.955815 & 41.755301 & 30.387857 \\ \text { C } & 36.531813 & 41.448652 & 31.708680 \\ \text { C } & 37.444175 & 40.958086 & 32.620382 \\ \text { C } & 38.816197 & 40.733203 & 32.303787 \\ \text { C } & 39.614066 & 40.158326 & 33.145379 \\ & 39.237192 & 41.105715 & 30.993774\end{array}$




$\begin{array}{llll}\text { C } & 38.333854 & 41.584033 & 30.074565 \\ \text { C } & 38.572420 & 41.949654 & 28.626997 \\ \text { C } & 37.155078 & 42.151647 & 28.038852 \\ \text { H } & 37.078199 & 43.098307 & 27.514576 \\ \text { H } & 36.897800 & 41.368324 & 27.325011 \\ \text { H } & 39.127805 & 41.181566 & 28.097812 \\ \text { H } & 39.147815 & 42.869138 & 28.550958 \\ \text { H } & 40.267573 & 40.947895 & 30.723909 \\ \text { H } & 37.113472 & 40.678490 & 33.604917 \\ \text { H } & 35.501406 & 41.566706 & 31.994539 \\ \text { H } & 34.181495 & 41.347270 & 28.948624 \\ \text { O } & 34.516041 & 44.543140 & 28.885825 \\ \text { H } & 30.430776 & 44.296938 & 28.957343 \\ \text { H } & 31.798251 & 44.757823 & 27.965955 \\ \text { H } & 30.556472 & 46.568789 & 29.134905 \\ \text { C } & 31.011419 & 46.016347 & 31.089878 \\ \text { O } & 29.887154 & 45.803719 & 31.406750 \\ \text { H } & 32.695910 & 47.759881 & 29.534426 \\ \text { H } & 31.762013 & 46.206675 & 31.796988 \\ \text { H } & 45.504849 & 39.992280 & 36.639144 \\ \text { H } & 41.448929 & 35.056028 & 33.768865\end{array}$

$\begin{array}{lrrr}\text { C7-C10-C11-C12 } 110^{\circ} & & \\ \mathrm{C} & 44.779973 & 39.841877 & 35.817311 \\ \mathrm{C} & 45.085679 & 39.078529 & 34.699913 \\ \mathrm{H} & 46.014023 & 38.541174 & 34.644811 \\ \mathrm{C} & 44.209138 & 38.989564 & 33.637052 \\ \mathrm{H} & 44.450938 & 38.401027 & 32.770437 \\ \mathrm{C} & 42.995647 & 39.669986 & 33.664286 \\ \mathrm{O} & 42.196717 & 39.582135 & 32.605365 \\ \mathrm{H} & 41.271722 & 39.856608 & 32.790145 \\ \mathrm{C} & 42.672170 & 40.418934 & 34.790175 \\ \mathrm{H} & 41.732360 & 40.938973 & 34.814579 \\ \mathrm{C} & 43.562996 & 40.505630 & 35.844185 \\ \mathrm{H} & 43.297088 & 41.115460 & 36.690482 \\ \mathrm{C} & 41.472012 & 35.860589 & 34.530345 \\ \mathrm{H} & 41.131932 & 35.497080 & 35.493933 \\ \mathrm{H} & 42.490467 & 36.218110 & 34.653890 \\ \mathrm{C} & 40.607145 & 37.038791 & 34.090649 \\ \mathrm{H} & 40.765056 & 37.863174 & 34.779733 \\ \mathrm{H} & 40.908933 & 37.432074 & 33.126602 \\ \mathrm{C} & 39.115930 & 36.801669 & 34.039943 \\ \mathrm{O} & 38.601010 & 35.746058 & 34.237481 \\ \mathrm{O} & 38.399086 & 37.863252 & 33.773295\end{array}$




\begin{tabular}{|c|c|c|c|}
\hline $\mathrm{H}$ & 38.920894 & 38.668688 & 33.582426 \\
\hline$N$ & 32.597132 & 46.894825 & 29.312932 \\
\hline $\mathrm{H}$ & 33.431213 & 46.380003 & 29.161453 \\
\hline C & 31.404929 & 46.159054 & 29.596192 \\
\hline C & 31.444689 & 44.760458 & 28.964277 \\
\hline$S$ & 32.356022 & 43.407677 & 29.788253 \\
\hline C & 34.058556 & 43.581582 & 29.207165 \\
\hline C & 34.817987 & 42.332305 & 29.244094 \\
\hline C & 36.273868 & 42.266355 & 29.318387 \\
\hline C & 37.006365 & 41.835134 & 30.437770 \\
\hline C & 36.600426 & 41.520559 & 31.763696 \\
\hline C & 37.517118 & 40.977454 & 32.641673 \\
\hline C & 38.873435 & 40.714596 & 32.287663 \\
\hline 0 & 39.672412 & 40.104410 & 33.102499 \\
\hline C & 39.273656 & 41.093253 & 30.974009 \\
\hline C & 38.367195 & 41.621019 & 30.087073 \\
\hline C & 38.584173 & 42.007719 & 28.643945 \\
\hline C & 37.152652 & 42.190300 & 28.085920 \\
\hline $\mathrm{H}$ & 37.065722 & 43.085831 & 27.478778 \\
\hline $\mathrm{H}$ & 36.857165 & 41.346236 & 27.458474 \\
\hline $\mathrm{H}$ & 39.156226 & 41.264351 & 28.098975 \\
\hline $\mathrm{H}$ & 39.133848 & 42.940337 & 28.575708 \\
\hline $\mathrm{H}$ & 40.287752 & 40.895164 & 30.673999 \\
\hline $\mathrm{H}$ & 37.199627 & 40.685750 & 33.627463 \\
\hline $\mathrm{H}$ & 35.581878 & 41.666629 & 32.077414 \\
\hline $\mathrm{H}$ & 34.255644 & 41.449904 & 28.992654 \\
\hline 0 & 34.503227 & 44.634686 & 28.831336 \\
\hline $\mathrm{H}$ & 30.435224 & 44.374437 & 28.929127 \\
\hline $\mathrm{H}$ & 31.808204 & 44.843051 & 27.950066 \\
\hline $\mathrm{H}$ & 30.555097 & 46.645465 & 29.119321 \\
\hline C & 31.002811 & 46.083910 & 31.074733 \\
\hline 0 & 29.873624 & 45.896031 & 31.386371 \\
\hline $\mathrm{H}$ & 32.686858 & 47.842145 & 29.543096 \\
\hline $\mathrm{H}$ & 31.758422 & 46.241512 & 31.785417 \\
\hline $\mathrm{H}$ & 45.513798 & 39.991796 & 36.622694 \\
\hline $\mathrm{H}$ & 41.477966 & 35.011188 & 33.811922 \\
\hline \multicolumn{4}{|c|}{ C7-C10-C11-C12 120 } \\
\hline C & 44.791175 & 39.828559 & 35.830082 \\
\hline C & 45.093601 & 39.064066 & 34.712992 \\
\hline $\mathrm{H}$ & 46.020168 & 38.523966 & 34.656672 \\
\hline C & 44.215522 & 38.975939 & 33.651482 \\
\hline $\mathrm{H}$ & 44.454727 & 38.385760 & 32.785246 \\
\hline C & 43.003399 & 39.657138 & 33.681133 \\
\hline
\end{tabular}




\begin{tabular}{|c|c|c|c|}
\hline 0 & 42.205709 & 39.574248 & 32.620647 \\
\hline $\mathrm{H}$ & 41.279718 & 39.839204 & 32.807051 \\
\hline C & 42.680414 & 40.403965 & 34.808836 \\
\hline $\mathrm{H}$ & 41.739170 & 40.920996 & 34.837340 \\
\hline C & 43.573261 & 40.490423 & 35.861333 \\
\hline $\mathrm{H}$ & 43.309156 & 41.097947 & 36.709667 \\
\hline C & 41.481749 & 35.877630 & 34.523161 \\
\hline $\mathrm{H}$ & 41.156244 & 35.500006 & 35.486431 \\
\hline $\mathrm{H}$ & 42.496391 & 36.247682 & 34.637951 \\
\hline C & 40.597026 & 37.044599 & 34.098517 \\
\hline $\mathrm{H}$ & 40.750314 & 37.869813 & 34.787037 \\
\hline $\mathrm{H}$ & 40.883202 & 37.442127 & 33.132111 \\
\hline C & 39.108932 & 36.788132 & 34.060450 \\
\hline 0 & 38.608086 & 35.725193 & 34.254279 \\
\hline 0 & 38.381580 & 37.844152 & 33.800169 \\
\hline $\mathrm{H}$ & 38.904501 & 38.648876 & 33.608726 \\
\hline $\mathrm{N}$ & 32.564273 & 46.899427 & 29.279554 \\
\hline $\mathrm{H}$ & 33.388345 & 46.372307 & 29.117072 \\
\hline C & 31.365307 & 46.180288 & 29.577284 \\
\hline C & 31.391247 & 44.777539 & 28.951854 \\
\hline$S$ & 32.278646 & 43.424222 & 29.799532 \\
\hline C & 33.988086 & 43.579759 & 29.249415 \\
\hline C & 34.760836 & 42.349495 & 29.398656 \\
\hline C & 36.222363 & 42.323926 & 29.451599 \\
\hline C & 36.974814 & 41.834165 & 30.532875 \\
\hline C & 36.594126 & 41.508539 & 31.861986 \\
\hline C & 37.521874 & 40.951526 & 32.715502 \\
\hline C & 38.868435 & 40.678400 & 32.328125 \\
\hline 0 & 39.676701 & 40.063135 & 33.125307 \\
\hline C & 39.242153 & 41.049694 & 31.004221 \\
\hline C & 38.321821 & 41.598049 & 30.143968 \\
\hline C & 38.501899 & 42.007022 & 28.702018 \\
\hline C & 37.059124 & 42.214020 & 28.188185 \\
\hline $\mathrm{H}$ & 36.971831 & 43.103620 & 27.574609 \\
\hline $\mathrm{H}$ & 36.726271 & 41.369577 & 27.581512 \\
\hline $\mathrm{H}$ & 39.051695 & 41.271575 & 28.122567 \\
\hline $\mathrm{H}$ & 39.055786 & 42.940378 & 28.639220 \\
\hline $\mathrm{H}$ & 40.247216 & 40.840788 & 30.679587 \\
\hline $\mathrm{H}$ & 37.225679 & 40.655821 & 33.706278 \\
\hline $\mathrm{H}$ & 35.583127 & 41.662959 & 32.195325 \\
\hline $\mathrm{H}$ & 34.240108 & 41.466232 & 29.064033 \\
\hline 0 & 34.432680 & 44.608711 & 28.811835 \\
\hline $\mathrm{H}$ & 30.377463 & 44.404934 & 28.908353 \\
\hline $\mathrm{H}$ & 31.768057 & 44.847256 & 27.941456 \\
\hline
\end{tabular}




$\begin{array}{llll}\mathrm{H} & 30.516992 & 46.673318 & 29.105235 \\ \mathrm{C} & 30.975178 & 46.116007 & 31.060109 \\ \mathrm{O} & 29.845138 & 45.944196 & 31.382050 \\ \mathrm{H} & 32.671002 & 47.844999 & 29.507877 \\ \mathrm{H} & 31.736947 & 46.268321 & 31.764754 \\ \mathrm{H} & 45.527368 & 39.975584 & 36.633655 \\ \mathrm{H} & 41.489339 & 35.035414 & 33.796771\end{array}$

$\begin{array}{llll}\text { C7-C10-C11-C12 130 } & & \\ \text { C } & 44.819584 & 39.821259 & 35.821539 \\ \text { C } & 45.141342 & 39.055268 & 34.711053 \\ \text { H } & 46.070855 & 38.519455 & 34.669712 \\ \text { C } & 44.279094 & 38.959454 & 33.637523 \\ \text { H } & 44.533478 & 38.368246 & 32.776355 \\ \text { C } & 43.063298 & 39.634029 & 33.648810 \\ \text { O } & 42.280389 & 39.542799 & 32.577770 \\ \text { H } & 41.353248 & 39.807740 & 32.751464 \\ \text { C } & 42.720243 & 40.381828 & 34.769847 \\ \text { H } & 41.775747 & 40.893375 & 34.784532 \\ \text { C } & 43.597893 & 40.476522 & 35.834262 \\ \text { H } & 43.318405 & 41.084772 & 36.677168 \\ \text { C } & 41.539783 & 35.881844 & 34.526067 \\ \text { H } & 41.232638 & 35.507895 & 35.496750 \\ \text { H } & 42.557141 & 36.250181 & 34.618483 \\ \text { C } & 40.649150 & 37.047598 & 34.114514 \\ \text { H } & 40.816874 & 37.875895 & 34.796095 \\ \text { H } & 40.915049 & 37.439345 & 33.140205 \\ \text { C } & 39.161606 & 36.790059 & 34.112359 \\ \text { O } & 38.664442 & 35.735306 & 34.351812 \\ \text { O } & 38.429585 & 37.838734 & 33.831683 \\ \text { H } & 38.954658 & 38.630864 & 33.608163 \\ \text { N } & 32.517810 & 46.900285 & 29.271541 \\ \text { H } & 33.338396 & 46.371852 & 29.097023 \\ \text { C } & 31.316189 & 46.182637 & 29.563233 \\ \text { C } & 31.346743 & 44.778753 & 28.939729 \\ \text { S } & 32.236800 & 43.437442 & 29.802581 \\ \text { C } & 33.951986 & 43.609698 & 29.278675 \\ \text { C } & 34.750081 & 42.412712 & 29.533696 \\ \text { C } & 36.216895 & 42.425737 & 29.567902 \\ \text { C } & 36.992842 & 41.873112 & 30.600390 \\ \text { C } & 36.642861 & 41.508157 & 31.928089 \\ \text { C } & 37.588121 & 40.924706 & 32.740857 \\ \text { C } & 38.925469 & 40.660519 & 32.310412 \\ \text { O } & 39.748353 & 40.027304 & 33.071371\end{array}$




\begin{tabular}{|c|c|c|c|}
\hline C & 39.265483 & 41.062306 & 30.985921 \\
\hline C & 38.326768 & 41.640355 & 30.167754 \\
\hline C & 38.466500 & 42.094093 & 28.736342 \\
\hline C & 37.009149 & 42.314267 & 28.273811 \\
\hline $\mathrm{H}$ & 36.901141 & 43.204906 & 27.664391 \\
\hline $\mathrm{H}$ & 36.648011 & 41.472084 & 27.679967 \\
\hline $\mathrm{H}$ & 39.004138 & 41.380893 & 28.118976 \\
\hline $\mathrm{H}$ & 39.015129 & 43.030437 & 28.689501 \\
\hline $\mathrm{H}$ & 40.259774 & 40.854000 & 30.629129 \\
\hline $\mathrm{H}$ & 37.315495 & 40.596153 & 33.728056 \\
\hline $\mathrm{H}$ & 35.638900 & 41.649993 & 32.286468 \\
\hline $\mathrm{H}$ & 34.287086 & 41.531207 & 29.110753 \\
\hline 0 & 34.378269 & 44.623182 & 28.790716 \\
\hline $\mathrm{H}$ & 30.334382 & 44.402397 & 28.894374 \\
\hline $\mathrm{H}$ & 31.727806 & 44.845422 & 27.930753 \\
\hline $\mathrm{H}$ & 30.469524 & 46.675944 & 29.088493 \\
\hline C & 30.920324 & 46.114753 & 31.044748 \\
\hline 0 & 29.790593 & 45.932630 & 31.361951 \\
\hline $\mathrm{H}$ & 32.628791 & 47.844647 & 29.502552 \\
\hline $\mathrm{H}$ & 31.677447 & 46.274919 & 31.752497 \\
\hline $\mathrm{H}$ & 45.544293 & 39.975421 & 36.634090 \\
\hline $\mathrm{H}$ & 41.530147 & 35.037972 & 33.801812 \\
\hline \multicolumn{4}{|c|}{$\mathrm{C} 7-\mathrm{C} 10-\mathrm{C} 11-\mathrm{C} 12140^{\circ}$} \\
\hline C & 44.870554 & 39.798346 & 35.776700 \\
\hline C & 45.199794 & 39.015049 & 34.680764 \\
\hline $\mathrm{H}$ & 46.121605 & 38.465885 & 34.660887 \\
\hline C & 44.352769 & 38.915031 & 33.595918 \\
\hline $\mathrm{H}$ & 44.611978 & 38.308845 & 32.746789 \\
\hline C & 43.144898 & 39.601496 & 33.583546 \\
\hline 0 & 42.372900 & 39.499325 & 32.503169 \\
\hline $\mathrm{H}$ & 41.450632 & 39.766811 & 32.667714 \\
\hline C & 42.793569 & 40.368085 & 34.688398 \\
\hline $\mathrm{H}$ & 41.853787 & 40.888622 & 34.684727 \\
\hline C & 43.656486 & 40.467192 & 35.764556 \\
\hline $\mathrm{H}$ & 43.371060 & 41.088352 & 36.595980 \\
\hline C & 41.643782 & 35.905317 & 34.585588 \\
\hline $\mathrm{H}$ & 41.375790 & 35.538864 & 35.570347 \\
\hline $\mathrm{H}$ & 42.670971 & 36.253567 & 34.625253 \\
\hline C & 40.751913 & 37.082403 & 34.214484 \\
\hline $\mathrm{H}$ & 40.988962 & 37.923691 & 34.858833 \\
\hline $\mathrm{H}$ & 40.941304 & 37.443365 & 33.211356 \\
\hline C & 39.268627 & 36.847632 & 34.355543 \\
\hline 0 & 38.792243 & 35.837407 & 34.764017 \\
\hline
\end{tabular}




$\begin{array}{llll}\text { O } & 38.516391 & 37.863621 & 34.008993 \\ \mathrm{H} & 39.026515 & 38.626011 & 33.685246 \\ \mathrm{~N} & 32.578131 & 46.929381 & 29.152637 \\ \mathrm{H} & 33.399112 & 46.393451 & 29.003756 \\ \mathrm{C} & 31.368817 & 46.220215 & 29.430826 \\ \mathrm{C} & 31.390698 & 44.819845 & 28.800885 \\ \mathrm{~S} & 32.225045 & 43.460846 & 29.687096 \\ \mathrm{C} & 33.969479 & 43.634741 & 29.259362 \\ \mathrm{C} & 34.757980 & 42.473994 & 29.634425 \\ \mathrm{C} & 36.234761 & 42.486649 & 29.643766 \\ \mathrm{C} & 37.021318 & 41.873570 & 30.625017 \\ \mathrm{C} & 36.689974 & 41.468750 & 31.948247 \\ \mathrm{C} & 37.646282 & 40.867813 & 32.729932 \\ \mathrm{C} & 38.980838 & 40.621120 & 32.269327 \\ \mathrm{O} & 39.816709 & 39.988532 & 33.002226 \\ \mathrm{C} & 39.297788 & 41.048378 & 30.943712 \\ \mathrm{C} & 38.347835 & 41.647280 & 30.159186 \\ \mathrm{C} & 38.457697 & 42.145001 & 28.740040 \\ \mathrm{C} & 36.991706 & 42.402983 & 28.325497 \\ \mathrm{H} & 36.879744 & 43.315630 & 27.751161 \\ \mathrm{H} & 36.599288 & 41.587959 & 27.713919 \\ \mathrm{H} & 38.969780 & 41.444495 & 28.087935 \\ \mathrm{H} & 39.018106 & 43.074864 & 28.709991 \\ \mathrm{H} & 40.288051 & 40.851510 & 30.569394 \\ \mathrm{H} & 37.393279 & 40.515286 & 33.713837 \\ \mathrm{H} & 35.690722 & 41.602749 & 32.321992 \\ \mathrm{H} & 34.321389 & 41.576590 & 29.212052 \\ \mathrm{O} & 34.405003 & 44.637101 & 28.750889 \\ \mathrm{H} & 30.370238 & 44.472391 & 28.730489 \\ \mathrm{H} & 31.799357 & 44.881783 & 27.802631 \\ \mathrm{H} & 30.532304 & 46.726345 & 28.952622 \\ \mathrm{H} & 30.960244 & 46.141854 & 30.907636 \\ \mathrm{H} & 29.822092 & 45.992134 & 31.214218 \\ \mathrm{H} & 32.690788 & 47.871873 & 29.388932 \\ \mathrm{H} & 31.719084 & 46.259309 & 31.622459 \\ \mathrm{H} & 41.579500 & 35.062166 & 33.863306\end{array}$

C7-C10-C11-C12 150

$\begin{array}{llll}\text { C } & 44.876092 & 39.814241 & 35.779089 \\ \text { C } & 45.211925 & 39.014184 & 34.697343 \\ \text { H } & 46.127322 & 38.454918 & 34.698031 \\ \text { C } & 44.378879 & 38.908202 & 33.602340 \\ \text { H } & 44.641353 & 38.287658 & 32.765002\end{array}$




\begin{tabular}{|c|c|c|c|}
\hline C & 43.182119 & 39.608645 & 33.569451 \\
\hline 0 & 42.417513 & 39.495461 & 32.476510 \\
\hline $\mathrm{H}$ & 41.520693 & 39.802514 & 32.626137 \\
\hline C & 42.824740 & 40.397089 & 34.653801 \\
\hline $\mathrm{H}$ & 41.894760 & 40.935077 & 34.629819 \\
\hline C & 43.672622 & 40.499809 & 35.742475 \\
\hline $\mathrm{H}$ & 43.383259 & 41.135452 & 36.561091 \\
\hline C & 41.689890 & 35.930675 & 34.600114 \\
\hline $\mathrm{H}$ & 41.472881 & 35.572768 & 35.600069 \\
\hline $\mathrm{H}$ & 42.716694 & 36.279894 & 34.583995 \\
\hline C & 40.776240 & 37.102925 & 34.266920 \\
\hline $\mathrm{H}$ & 41.075451 & 37.964402 & 34.856888 \\
\hline $\mathrm{H}$ & 40.871124 & 37.422961 & 33.236079 \\
\hline C & 39.315274 & 36.881328 & 34.564271 \\
\hline 0 & 38.881777 & 35.914502 & 35.095270 \\
\hline 0 & 38.520060 & 37.871766 & 34.211424 \\
\hline $\mathrm{H}$ & 38.991426 & 38.613107 & 33.825933 \\
\hline $\mathrm{N}$ & 32.507563 & 46.896814 & 29.221681 \\
\hline $\mathrm{H}$ & 33.296804 & 46.334834 & 29.001381 \\
\hline C & 31.273770 & 46.236344 & 29.507047 \\
\hline C & 31.272460 & 44.813642 & 28.936734 \\
\hline$S$ & 32.168639 & 43.534303 & 29.864777 \\
\hline C & 33.890350 & 43.624629 & 29.235510 \\
\hline C & 34.672524 & 42.596378 & 29.785618 \\
\hline C & 36.100347 & 42.416965 & 29.593432 \\
\hline C & 36.910303 & 41.818529 & 30.581299 \\
\hline C & 36.544617 & 41.303726 & 31.863735 \\
\hline C & 37.515796 & 40.745227 & 32.666161 \\
\hline C & 38.888886 & 40.661099 & 32.255873 \\
\hline 0 & 39.752230 & 40.084094 & 32.966100 \\
\hline C & 39.240934 & 41.223532 & 30.961945 \\
\hline C & 38.277039 & 41.771837 & 30.162841 \\
\hline C & 38.393656 & 42.416465 & 28.796888 \\
\hline C & 36.929030 & 42.691405 & 28.362815 \\
\hline $\mathrm{H}$ & 36.789484 & 43.698193 & 27.990090 \\
\hline $\mathrm{H}$ & 36.627955 & 42.016416 & 27.561383 \\
\hline $\mathrm{H}$ & 38.922447 & 41.783365 & 28.090860 \\
\hline $\mathrm{H}$ & 38.954371 & 43.343930 & 28.868755 \\
\hline $\mathrm{H}$ & 40.271635 & 41.162782 & 30.658106 \\
\hline $\mathrm{H}$ & 37.258413 & 40.321607 & 33.619677 \\
\hline $\mathrm{H}$ & 35.527858 & 41.355403 & 32.200444 \\
\hline $\mathrm{H}$ & 34.186090 & 41.942069 & 30.480635 \\
\hline 0 & 34.216136 & 44.510420 & 28.453027 \\
\hline $\mathrm{H}$ & 30.251487 & 44.461490 & 28.921933 \\
\hline
\end{tabular}




$\begin{array}{llll}\mathrm{H} & 31.646317 & 44.832152 & 27.922847 \\ \mathrm{H} & 30.450823 & 46.750041 & 29.011594 \\ \mathrm{C} & 30.866266 & 46.206979 & 30.986211 \\ \mathrm{O} & 29.723558 & 46.114024 & 31.304005 \\ \mathrm{H} & 32.656164 & 47.836842 & 29.446018 \\ \mathrm{H} & 31.633426 & 46.299773 & 31.695598 \\ \mathrm{H} & 45.580500 & 39.966489 & 36.609193 \\ \mathrm{H} & 41.589984 & 35.082091 & 33.887980\end{array}$

C7-C10-C11-C12 160

$\begin{array}{llll}\text { C } & 44.874816 & 39.804371 & 35.775837 \\ \text { C } & 45.216287 & 38.994514 & 34.703161 \\ \text { H } & 46.129461 & 38.432284 & 34.715654 \\ \text { C } & 44.392000 & 38.882735 & 33.602453 \\ \text { H } & 44.659384 & 38.255680 & 32.771602 \\ \text { C } & 43.197824 & 39.586266 & 33.555459 \\ \text { O } & 42.441997 & 39.466106 & 32.456414 \\ \text { H } & 41.546814 & 39.778334 & 32.594304 \\ \text { C } & 42.833458 & 40.383048 & 34.630865 \\ \text { H } & 41.904989 & 40.923263 & 34.596861 \\ \text { C } & 43.673552 & 40.492561 & 35.725100 \\ \text { H } & 43.379725 & 41.135723 & 36.536191 \\ \mathrm{C} & 41.699530 & 35.927014 & 34.598819 \\ \text { H } & 41.488244 & 35.571830 & 35.600765 \\ \text { H } & 42.725832 & 36.276763 & 34.575444 \\ \mathrm{C} & 40.782176 & 37.097358 & 34.269097 \\ \text { H } & 41.088839 & 37.962594 & 34.849885 \\ \text { H } & 40.862418 & 37.410708 & 33.234997 \\ \mathrm{C} & 39.326131 & 36.878322 & 34.591143 \\ \text { O } & 38.901544 & 35.924793 & 35.151224 \\ \text { O } & 38.523831 & 37.860190 & 34.226382 \\ \text { H } & 38.991762 & 38.590465 & 33.820008 \\ \text { N } & 32.493254 & 46.896297 & 29.224809 \\ \text { H } & 33.270825 & 46.330051 & 28.976757 \\ \text { C } & 31.250995 & 46.251155 & 29.510657 \\ \text { C } & 31.226611 & 44.829530 & 28.939483 \\ \text { S } & 32.119077 & 43.543131 & 29.857866 \\ \text { C } & 33.838117 & 43.634877 & 29.209677 \\ \text { C } & 34.659433 & 42.706466 & 29.872542 \\ \text { C } & 36.050727 & 42.474959 & 29.642724 \\ \text { C } & 36.882747 & 41.836666 & 30.614497 \\ \text { C } & 36.540235 & 41.313772 & 31.895629 \\ \text { C } & 37.520384 & 40.730431 & 32.671912 \\ \text { C } & 38.884207 & 40.640517 & 32.231775\end{array}$




$\begin{array}{rrrr}\text { O } & 39.758349 & 40.053974 & 32.918908 \\ \text { C } & 39.213164 & 41.218131 & 30.940267 \\ \text { C } & 38.234115 & 41.78409 & 30.167296 \\ \text { C } & 38.336978 & 42.457838 & 28.815009 \\ \text { C } & 36.869851 & 42.753022 & 28.406205 \\ \text { H } & 36.735991 & 43.761372 & 28.037275 \\ \text { H } & 36.554838 & 42.08515 & 27.604674 \\ \text { H } & 38.852104 & 41.838364 & 28.087543 \\ \text { H } & 38.907985 & 43.377362 & 28.903986 \\ \text { H } & 40.237198 & 41.156525 & 30.615133 \\ \text { H } & 37.278159 & 40.301864 & 33.627023 \\ \text { H } & 35.532934 & 41.372662 & 32.257804 \\ \text { H } & 34.208991 & 42.186902 & 30.695125 \\ \text { O } & 34.112511 & 44.432842 & 28.319001 \\ \text { H } & 30.200813 & 44.491115 & 28.93312 \\ \text { H } & 31.593314 & 44.8429 & 27.923216 \\ \text { H } & 30.433323 & 46.777603 & 29.019444 \\ \text { C } & 30.848559 & 46.223471 & 30.99106 \\ \text { O } & 29.705649 & 46.140609 & 31.311998 \\ \text { H } & 32.65253 & 47.834796 & 29.44857 \\ \text { H } & 31.618369 & 46.308757 & 31.698748 \\ \text { H } & 45.574685 & 39.963503 & 36.608392 \\ \text { H } & 41.595471 & 35.077252 & 33.888753\end{array}$

$\begin{array}{lrrr}\text { C7-C10-C11-C12 170 } & & \\ \text { C } & 44.871428 & 39.7981 & 35.770433 \\ \text { C } & 45.218515 & 38.983739 & 34.702821 \\ \text { H } & 46.132406 & 38.422744 & 34.721984 \\ \text { C } & 44.398841 & 38.865242 & 33.599209 \\ \text { H } & 44.67001 & 38.233546 & 32.773118 \\ \text { C } & 43.204117 & 39.567118 & 33.543717 \\ \text { O } & 42.452187 & 39.439189 & 32.442231 \\ \text { H } & 41.556127 & 39.748932 & 32.575446 \\ \text { C } & 42.835293 & 40.370116 & 34.61269 \\ \text { H } & 41.907251 & 40.910597 & 34.571317 \\ \text { C } & 43.670094 & 40.485358 & 35.710554 \\ \text { H } & 43.371911 & 41.132121 & 36.517159 \\ \text { C } & 41.700068 & 35.923625 & 34.600616 \\ \text { H } & 41.49237 & 35.565292 & 35.602207 \\ \text { H } & 42.725472 & 36.275623 & 34.57538 \\ \text { C } & 40.778787 & 37.091334 & 34.275899 \\ \text { H } & 41.086303 & 37.956983 & 34.855602 \\ \text { H } & 40.852723 & 37.403906 & 33.24132 \\ \text { C } & 39.325454 & 36.869426 & 34.606895\end{array}$




\begin{tabular}{|c|c|c|c|}
\hline 0 & 38.908755 & 35.923421 & 35.184766 \\
\hline 0 & 38.516542 & 37.841514 & 34.229309 \\
\hline $\mathrm{H}$ & 38.981625 & 38.566829 & 33.811693 \\
\hline $\mathrm{N}$ & 32.488343 & 46.886638 & 29.223175 \\
\hline $\mathrm{H}$ & 33.26143 & 46.318122 & 28.968394 \\
\hline C & 31.240466 & 46.251237 & 29.508531 \\
\hline C & 31.201166 & 44.831516 & 28.935217 \\
\hline S & 32.091021 & 43.540304 & 29.847794 \\
\hline C & 33.809164 & 43.645098 & 29.201983 \\
\hline C & 34.666015 & 42.818239 & 29.950907 \\
\hline C & 36.041534 & 42.570492 & 29.704521 \\
\hline C & 36.875271 & 41.876904 & 30.649282 \\
\hline C & 36.542005 & 41.340076 & 31.923522 \\
\hline C & 37.521277 & 40.724183 & 32.677048 \\
\hline C & 38.876659 & 40.617201 & 32.215596 \\
\hline 0 & 39.752939 & 40.018316 & 32.889227 \\
\hline C & 39.194237 & 41.195435 & 30.923111 \\
\hline C & 38.212187 & 41.790961 & 30.172287 \\
\hline C & 38.308199 & 42.479735 & 28.826158 \\
\hline C & 36.839573 & 42.801406 & 28.444191 \\
\hline $\mathrm{H}$ & 36.721876 & 43.80234 & 28.050651 \\
\hline $\mathrm{H}$ & 36.492074 & 42.119747 & 27.667781 \\
\hline $\mathrm{H}$ & 38.802463 & 41.861409 & 28.08371 \\
\hline $\mathrm{H}$ & 38.893046 & 43.391009 & 28.918264 \\
\hline $\mathrm{H}$ & 40.211753 & 41.119405 & 30.580924 \\
\hline $\mathrm{H}$ & 37.285679 & 40.290267 & 33.631314 \\
\hline $\mathrm{H}$ & 35.542324 & 41.41418 & 32.303367 \\
\hline $\mathrm{H}$ & 34.237426 & 42.37728 & 30.82944 \\
\hline 0 & 34.052835 & 44.361259 & 28.235432 \\
\hline $\mathrm{H}$ & 30.173196 & 44.501191 & 28.93594 \\
\hline $\mathrm{H}$ & 31.562085 & 44.842296 & 27.916799 \\
\hline $\mathrm{H}$ & 30.427469 & 46.787007 & 29.019787 \\
\hline C & 30.840578 & 46.224036 & 30.989834 \\
\hline 0 & 29.698238 & 46.144253 & 31.31346 \\
\hline $\mathrm{H}$ & 32.651514 & 47.825288 & 29.44339 \\
\hline $\mathrm{H}$ & 31.612136 & 46.307921 & 31.695744 \\
\hline $\mathrm{H}$ & 45.56731 & 39.961674 & 36.605668 \\
\hline $\mathrm{H}$ & 41.59589 & 35.075066 & 33.889215 \\
\hline \multicolumn{4}{|c|}{ C7-C10-C11-C12 180 } \\
\hline C & 44.859492 & 39.789413 & 35.762635 \\
\hline C & 45.212253 & 38.971144 & 34.699872 \\
\hline $\mathrm{H}$ & 46.126422 & 38.410842 & 34.725624 \\
\hline C & 44.398611 & 38.849002 & 33.592421 \\
\hline
\end{tabular}




\begin{tabular}{|c|c|c|c|}
\hline $\mathrm{H}$ & 44.675152 & 38.216184 & 32.769001 \\
\hline C & 43.203468 & 39.549671 & 33.528654 \\
\hline 0 & 42.458628 & 39.41833 & 32.422968 \\
\hline $\mathrm{H}$ & 41.561995 & 39.729408 & 32.546252 \\
\hline C & 42.826953 & 40.352888 & 34.594505 \\
\hline $\mathrm{H}$ & 41.897179 & 40.890237 & 34.549238 \\
\hline C & 43.656717 & 40.473183 & 35.695717 \\
\hline $\mathrm{H}$ & 43.353481 & 41.121999 & 36.498797 \\
\hline C & 41.700846 & 35.932544 & 34.599553 \\
\hline $\mathrm{H}$ & 41.495522 & 35.571641 & 35.600564 \\
\hline $\mathrm{H}$ & 42.726029 & 36.284673 & 34.571492 \\
\hline C & 40.777114 & 37.100546 & 34.282258 \\
\hline $\mathrm{H}$ & 41.090479 & 37.966536 & 34.858502 \\
\hline $\mathrm{H}$ & 40.840357 & 37.412391 & 33.247008 \\
\hline C & 39.327927 & 36.880743 & 34.632517 \\
\hline 0 & 38.920728 & 35.947969 & 35.238203 \\
\hline 0 & 38.512735 & 37.842244 & 34.241161 \\
\hline $\mathrm{H}$ & 38.97354 & 38.554057 & 33.796773 \\
\hline $\mathrm{N}$ & 32.46638 & 46.871022 & 29.240506 \\
\hline $\mathrm{H}$ & 33.237278 & 46.30173 & 28.983033 \\
\hline C & 31.216206 & 46.240734 & 29.532452 \\
\hline C & 31.165814 & 44.819274 & 28.96366 \\
\hline$S$ & 32.05286 & 43.528054 & 29.879859 \\
\hline C & 33.762797 & 43.629251 & 29.207833 \\
\hline C & 34.661793 & 42.913412 & 30.020971 \\
\hline C & 36.026592 & 42.647767 & 29.755407 \\
\hline C & 36.856504 & 41.898482 & 30.667646 \\
\hline C & 36.540285 & 41.358548 & 31.944204 \\
\hline C & 37.523732 & 40.718889 & 32.673778 \\
\hline C & 38.867451 & 40.58977 & 32.183818 \\
\hline 0 & 39.748111 & 39.978476 & 32.840087 \\
\hline C & 39.165276 & 41.164384 & 30.886823 \\
\hline C & 38.178059 & 41.781085 & 30.15946 \\
\hline C & 38.266533 & 42.484288 & 28.822886 \\
\hline C & 36.802844 & 42.856664 & 28.476622 \\
\hline $\mathrm{H}$ & 36.70779 & 43.861872 & 28.087509 \\
\hline $\mathrm{H}$ & 36.415223 & 42.190906 & 27.706235 \\
\hline $\mathrm{H}$ & 38.725686 & 41.862908 & 28.060511 \\
\hline $\mathrm{H}$ & 38.883535 & 43.372909 & 28.9162 \\
\hline $\mathrm{H}$ & 40.172704 & 41.071105 & 30.520228 \\
\hline $\mathrm{H}$ & 37.300387 & 40.290383 & 33.633468 \\
\hline $\mathrm{H}$ & 35.550075 & 41.446183 & 32.34594 \\
\hline $\mathrm{H}$ & 34.256123 & 42.527199 & 30.935539 \\
\hline 0 & 33.967538 & 44.263011 & 28.177095 \\
\hline
\end{tabular}




$\begin{array}{rrrr}\text { H } & 30.135396 & 44.497442 & 28.964276 \\ \text { H } & 31.528886 & 44.822222 & 27.945936 \\ \text { H } & 30.405188 & 46.780834 & 29.045411 \\ \mathrm{C} & 30.819979 & 46.2215 & 31.015005 \\ \mathrm{O} & 29.678251 & 46.140816 & 31.340412 \\ \mathrm{H} & 32.631558 & 47.810492 & 29.456516 \\ \mathrm{H} & 31.592174 & 46.312797 & 31.719386 \\ \mathrm{H} & 45.552624 & 39.959625 & 36.598847 \\ \mathrm{H} & 41.594098 & 35.086885 & 33.885178\end{array}$

$\begin{array}{llll}\text { C7-C10-C11-C12 } 0^{\circ} & & \\ \text { C } & 44.824667 & 39.846192 & 35.783472 \\ \text { C } & 45.150378 & 39.056024 & 34.690965 \\ \text { H } & 46.065111 & 38.495369 & 34.678461 \\ \text { C } & 44.309990 & 38.966155 & 33.599622 \\ \text { H } & 44.566348 & 38.356162 & 32.752674 \\ \text { C } & 43.117088 & 39.673995 & 33.580436 \\ \text { O } & 42.351530 & 39.582872 & 32.484849 \\ \text { H } & 41.466617 & 39.921642 & 32.624944 \\ \text { C } & 42.767059 & 40.447511 & 34.677144 \\ \text { H } & 41.839443 & 40.990440 & 34.666359 \\ \text { C } & 43.622491 & 40.534711 & 35.761545 \\ \text { H } & 43.340713 & 41.163047 & 36.588238 \\ \text { C } & 41.626458 & 35.945549 & 34.549418 \\ \text { H } & 41.361330 & 35.584306 & 35.536689 \\ \text { H } & 42.648360 & 36.307887 & 34.589041 \\ \text { C } & 40.713147 & 37.105414 & 34.170027 \\ \text { H } & 40.934249 & 37.956085 & 34.808637 \\ \text { H } & 40.890747 & 37.458510 & 33.160336 \\ \text { C } & 39.237026 & 36.834067 & 34.318235 \\ \text { O } & 38.780458 & 35.810628 & 34.702182 \\ \text { O } & 38.451899 & 37.846109 & 34.003163 \\ \text { H } & 38.931425 & 38.618051 & 33.704331 \\ \text { N } & 32.663747 & 46.838755 & 29.418096 \\ \text { H } & 33.504848 & 46.312917 & 29.451709 \\ \text { C } & 31.442889 & 46.128921 & 29.702817 \\ \text { C } & 31.534128 & 44.669079 & 29.193660 \\ \text { S } & 31.760380 & 43.347462 & 30.430621 \\ \text { C } & 33.493141 & 42.752432 & 30.620107 \\ \text { C } & 34.480650 & 42.991033 & 29.631429 \\ \text { C } & 35.847419 & 42.634730 & 29.642082 \\ \text { C } & 36.684313 & 41.930074 & 30.630171 \\ \text { C } & 36.365813 & 41.280863 & 31.847992\end{array}$




$\begin{array}{llll}\text { C } & 37.375231 & 40.724961 & 32.617516 \\ \text { C } & 38.753281 & 40.766120 & 32.224722 \\ \text { O } & 39.651254 & 40.213352 & 32.913281 \\ \text { C } & 39.056763 & 41.436265 & 30.985523 \\ \text { C } & 38.042936 & 41.980006 & 30.220454 \\ \text { C } & 38.180747 & 42.750555 & 28.930403 \\ \text { C } & 36.729319 & 42.907965 & 28.438696 \\ \text { H } & 36.547888 & 43.888933 & 28.008748 \\ \text { H } & 36.523574 & 42.172955 & 27.658696 \\ \text { H } & 38.812173 & 42.243355 & 28.208557 \\ \text { H } & 38.634205 & 43.719419 & 29.124444 \\ \text { H } & 40.085047 & 41.475643 & 30.670178 \\ \text { H } & 37.132317 & 40.224673 & 33.536718 \\ \text { H } & 35.351681 & 41.225592 & 32.173255 \\ \text { H } & 34.153810 & 43.518721 & 28.753978 \\ \text { O } & 33.559334 & 42.095453 & 31.649784 \\ \text { H } & 30.587883 & 44.415080 & 28.736059 \\ \text { H } & 32.279978 & 44.614350 & 28.413140 \\ \text { H } & 30.636044 & 46.599644 & 29.147734 \\ \text { C } & 30.993030 & 46.219038 & 31.168279 \\ \text { O } & 29.840393 & 46.250786 & 31.455830 \\ \text { H } & 32.741915 & 47.800893 & 29.588270 \\ \text { H } & 31.749146 & 46.312631 & 31.889831 \\ \text { H } & 45.539212 & 39.997924 & 36.605701 \\ \text { H } & 41.574662 & 35.095195 & 33.834344\end{array}$

$\begin{array}{lrrr}\text { C7-C10-C11-C12 - } 10^{\circ} & & \\ \text { C } & 44.836710 & 39.848833 & 35.793965 \\ \text { C } & 45.158029 & 39.057588 & 34.700755 \\ \text { H } & 46.072696 & 38.496717 & 34.685105 \\ \text { C } & 44.314227 & 38.968958 & 33.612085 \\ \text { H } & 44.567682 & 38.359515 & 32.763872 \\ \text { C } & 43.121907 & 39.678528 & 33.596439 \\ \text { O } & 42.352867 & 39.588924 & 32.503676 \\ \text { H } & 41.471231 & 39.936874 & 32.644351 \\ \text { C } & 42.776071 & 40.451954 & 34.694384 \\ \text { H } & 41.849165 & 40.996057 & 34.686246 \\ \text { C } & 43.635207 & 40.538231 & 35.775974 \\ \text { H } & 43.356758 & 41.167086 & 36.603389 \\ \text { C } & 41.620322 & 35.947955 & 34.559177 \\ \text { H } & 41.345559 & 35.583103 & 35.542517 \\ \text { H } & 42.640941 & 36.312738 & 34.610981 \\ \text { C } & 40.711239 & 37.109281 & 34.172499 \\ \text { H } & 40.923832 & 37.957128 & 34.817709\end{array}$




\begin{tabular}{|c|c|c|c|}
\hline $\mathrm{H}$ & 40.904181 & 37.467224 & 33.167293 \\
\hline C & 39.232377 & 36.840158 & 34.292813 \\
\hline 0 & 38.766360 & 35.810764 & 34.648507 \\
\hline 0 & 38.455191 & 37.860492 & 33.984078 \\
\hline $\mathrm{H}$ & 38.940094 & 38.638186 & 33.709933 \\
\hline $\mathrm{N}$ & 32.680145 & 46.794694 & 29.381340 \\
\hline $\mathrm{H}$ & 33.517893 & 46.264738 & 29.430593 \\
\hline C & 31.453470 & 46.096762 & 29.671033 \\
\hline C & 31.536322 & 44.631767 & 29.177728 \\
\hline$S$ & 31.765748 & 43.325518 & 30.429848 \\
\hline C & 33.490053 & 42.696641 & 30.589698 \\
\hline C & 34.498886 & 43.011531 & 29.647327 \\
\hline C & 35.859165 & 42.617853 & 29.646635 \\
\hline C & 36.699826 & 41.955466 & 30.655111 \\
\hline C & 36.391222 & 41.375972 & 31.912470 \\
\hline C & 37.400943 & 40.822308 & 32.682659 \\
\hline C & 38.770707 & 40.807805 & 32.261435 \\
\hline 0 & 39.667543 & 40.250526 & 32.948205 \\
\hline C & 39.068402 & 41.432516 & 30.996605 \\
\hline C & 38.054705 & 41.971016 & 30.228326 \\
\hline C & 38.190072 & 42.688616 & 28.907383 \\
\hline C & 36.734859 & 42.880676 & 28.436900 \\
\hline $\mathrm{H}$ & 36.569341 & 43.869589 & 28.017804 \\
\hline $\mathrm{H}$ & 36.500141 & 42.160194 & 27.652352 \\
\hline $\mathrm{H}$ & 38.786611 & 42.131836 & 28.191990 \\
\hline $\mathrm{H}$ & 38.683507 & 43.645841 & 29.054450 \\
\hline $\mathrm{H}$ & 40.091860 & 41.437663 & 30.663700 \\
\hline $\mathrm{H}$ & 37.165281 & 40.365352 & 33.626287 \\
\hline $\mathrm{H}$ & 35.381788 & 41.354237 & 32.256375 \\
\hline $\mathrm{H}$ & 34.188804 & 43.582755 & 28.791591 \\
\hline 0 & 33.532755 & 41.963545 & 31.566593 \\
\hline $\mathrm{H}$ & 30.587067 & 44.375588 & 28.727468 \\
\hline $\mathrm{H}$ & 32.278311 & 44.564766 & 28.394585 \\
\hline $\mathrm{H}$ & 30.650301 & 46.567359 & 29.110737 \\
\hline C & 31.005196 & 46.200269 & 31.135776 \\
\hline 0 & 29.853612 & 46.250126 & 31.425397 \\
\hline $\mathrm{H}$ & 32.762478 & 47.759261 & 29.534600 \\
\hline $\mathrm{H}$ & 31.763728 & 46.284624 & 31.855436 \\
\hline $\mathrm{H}$ & 45.552841 & 39.998889 & 36.614847 \\
\hline $\mathrm{H}$ & 41.579278 & 35.099431 & 33.841350 \\
\hline \multicolumn{4}{|c|}{$\mathrm{C} 7-\mathrm{C} 10-\mathrm{C} 11-\mathrm{C} 12-20^{\circ}$} \\
\hline C & 44.826009 & 39.842570 & 35.811454 \\
\hline C & 45.153125 & 39.050485 & 34.720720 \\
\hline
\end{tabular}




$\begin{array}{llll}\text { H } & 46.068393 & 38.490379 & 34.710192 \\ \mathrm{C} & 44.314574 & 38.959139 & 33.628335 \\ \mathrm{H} & 44.572226 & 38.347719 & 32.782790 \\ \mathrm{C} & 43.121691 & 39.667751 & 33.605356 \\ \mathrm{O} & 42.359988 & 39.578080 & 32.508321 \\ \mathrm{H} & 41.475829 & 39.926278 & 32.642257 \\ \mathrm{C} & 42.769645 & 40.442110 & 34.701337 \\ \mathrm{H} & 41.841645 & 40.984070 & 34.687418 \\ \mathrm{C} & 43.623379 & 40.530461 & 35.787068 \\ \mathrm{H} & 43.340453 & 41.160376 & 36.612215 \\ \mathrm{C} & 41.555010 & 35.944574 & 34.538204 \\ \mathrm{H} & 41.244172 & 35.579248 & 35.510621 \\ \mathrm{H} & 42.574408 & 36.306357 & 34.628474 \\ \mathrm{C} & 40.666042 & 37.111890 & 34.120272 \\ \mathrm{H} & 40.853532 & 37.953340 & 34.781140 \\ \mathrm{H} & 40.908599 & 37.478427 & 33.128905 \\ \mathrm{C} & 39.180240 & 36.851398 & 34.163511 \\ \mathrm{O} & 38.689696 & 35.810910 & 34.449164 \\ \mathrm{O} & 38.425308 & 37.890604 & 33.865615 \\ \mathrm{H} & 38.924141 & 38.674519 & 33.634970 \\ \mathrm{H} & 32.699582 & 46.795348 & 29.389435 \\ \mathrm{H} & 33.545706 & 46.279596 & 29.444045 \\ \mathrm{H} & 31.485730 & 46.077214 & 29.681130 \\ \mathrm{H} & 31.593660 & 44.611436 & 29.198775 \\ \mathrm{C} & 31.904563 & 43.323440 & 30.455344 \\ \mathrm{C} & 33.625744 & 42.664402 & 30.460604 \\ \mathrm{H} & 34.638626 & 43.148357 & 29.602098 \\ \mathrm{C} & 36.016058 & 42.752553 & 29.604068 \\ \mathrm{C} & 36.827048 & 42.115161 & 30.622682 \\ \mathrm{C} & 36.524101 & 41.700616 & 31.954463 \\ \mathrm{C} & 37.500785 & 41.093516 & 32.721895 \\ \mathrm{C} & 38.837092 & 40.890123 & 32.250416 \\ \mathrm{C} & 39.701305 & 40.282417 & 32.936440 \\ \mathrm{C} & 38.146571 & 41.393984 & 30.930045 \\ \mathrm{C} & 4165314 & 41.973432 & 30.156318 \\ \mathrm{C} & 42.510562 & 42.561216 & 28.772201 \\ \mathrm{H} & 42.933487 & 28.365481 \\ \mathrm{H} & 43.944430 & 27.970176 \\ \mathrm{H} & 42.266613 & 27.582209 \\ \mathrm{H} & 41.859897 & 28.077667 \\ \mathrm{H} & 43.437295 & 28.797920 \\ \mathrm{H} & 41.271207 & 30.568173 \\ \mathrm{H} & 30.733356 & 33.706651 \\ \mathrm{H} & 320069 & 32.340798\end{array}$




\begin{tabular}{|c|c|c|c|}
\hline $\mathrm{H}$ & 34.346319 & 43.830238 & 28.824990 \\
\hline 0 & 33.689684 & 41.770270 & 31.288412 \\
\hline $\mathrm{H}$ & 30.638829 & 44.323987 & 28.780639 \\
\hline $\mathrm{H}$ & 32.314608 & 44.553419 & 28.395603 \\
\hline $\mathrm{H}$ & 30.673797 & 46.529648 & 29.118631 \\
\hline C & 31.035312 & 46.171995 & 31.144705 \\
\hline 0 & 29.883375 & 46.190495 & 31.435779 \\
\hline $\mathrm{H}$ & 32.766780 & 47.761438 & 29.540022 \\
\hline $\mathrm{H}$ & 31.792119 & 46.272728 & 31.863494 \\
\hline $\mathrm{H}$ & 45.540116 & 39.995738 & 36.633670 \\
\hline $\mathrm{H}$ & 41.539347 & 35.096693 & 33.818492 \\
\hline \multicolumn{4}{|c|}{ C7-C10-C11-C12-30 } \\
\hline C & 44.763707 & 39.866204 & 35.794006 \\
\hline C & 45.068167 & 39.090094 & 34.684973 \\
\hline $\mathrm{H}$ & 45.990334 & 38.541455 & 34.640688 \\
\hline C & 44.198790 & 39.002429 & 33.616349 \\
\hline $\mathrm{H}$ & 44.439057 & 38.403663 & 32.756611 \\
\hline C & 42.995862 & 39.697335 & 33.632749 \\
\hline 0 & 42.206089 & 39.610046 & 32.559405 \\
\hline $\mathrm{H}$ & 41.301847 & 39.909074 & 32.720662 \\
\hline C & 42.670009 & 40.457490 & 34.747587 \\
\hline $\mathrm{H}$ & 41.736505 & 40.989768 & 34.764524 \\
\hline C & 43.553984 & 40.542540 & 35.809292 \\
\hline $\mathrm{H}$ & 43.288848 & 41.161013 & 36.648988 \\
\hline C & 41.493262 & 35.951995 & 34.523110 \\
\hline $\mathrm{H}$ & 41.187954 & 35.583009 & 35.496173 \\
\hline $\mathrm{H}$ & 42.506397 & 36.331565 & 34.613773 \\
\hline C & 40.585771 & 37.104899 & 34.100744 \\
\hline $\mathrm{H}$ & 40.760370 & 37.950421 & 34.759601 \\
\hline $\mathrm{H}$ & 40.824931 & 37.473542 & 33.109811 \\
\hline C & 39.102186 & 36.828559 & 34.140785 \\
\hline 0 & 38.627435 & 35.775266 & 34.415696 \\
\hline 0 & 38.336727 & 37.858720 & 33.857658 \\
\hline $\mathrm{H}$ & 38.818247 & 38.656804 & 33.609569 \\
\hline $\mathrm{N}$ & 32.723616 & 46.771675 & 29.426025 \\
\hline $\mathrm{H}$ & 33.596713 & 46.304857 & 29.425342 \\
\hline C & 31.558692 & 45.982533 & 29.727770 \\
\hline C & 31.745373 & 44.520991 & 29.253420 \\
\hline$S$ & 32.242370 & 43.264862 & 30.496009 \\
\hline C & 33.891489 & 42.585539 & 30.042872 \\
\hline C & 34.903083 & 43.399313 & 29.474963 \\
\hline C & 36.344976 & 43.031666 & 29.534103 \\
\hline C & 36.993667 & 42.297365 & 30.520011 \\
\hline
\end{tabular}




$\begin{array}{llll}\text { C } & 36.572201 & 41.877640 & 31.831883 \\ \text { C } & 37.441288 & 41.176985 & 32.631564 \\ \text { C } & 38.773786 & 40.847538 & 32.236624 \\ \text { O } & 39.558929 & 40.181400 & 32.974368 \\ \text { C } & 39.194502 & 41.296043 & 30.921613 \\ \text { C } & 38.344492 & 41.984068 & 30.115391 \\ \text { C } & 38.588148 & 42.558890 & 28.739659 \\ \text { C } & 37.186721 & 43.004352 & 28.270491 \\ \text { H } & 37.208291 & 43.964355 & 27.761435 \\ \text { H } & 36.759454 & 42.283031 & 27.572762 \\ \text { H } & 39.053498 & 41.847316 & 28.065067 \\ \text { H } & 39.253483 & 43.415850 & 28.805452 \\ \text { H } & 40.191984 & 41.044434 & 30.603674 \\ \text { H } & 37.120770 & 40.834765 & 33.599791 \\ \text { H } & 35.590094 & 42.114793 & 32.185629 \\ \text { H } & 34.693822 & 44.454591 & 29.464412 \\ \text { O } & 34.030356 & 41.415338 & 30.363131 \\ \text { H } & 30.794922 & 44.165464 & 28.881815 \\ \text { H } & 32.441102 & 44.501201 & 28.425796 \\ \text { H } & 30.717913 & 46.375373 & 29.163157 \\ \text { C } & 31.101483 & 46.062459 & 31.189742 \\ \text { O } & 29.952374 & 45.981970 & 31.477252 \\ \text { H } & 32.747312 & 47.738032 & 29.587719 \\ \text { H } & 31.843305 & 46.234919 & 31.910200 \\ \text { H } & 45.496837 & 40.014069 & 36.600760 \\ \text { H } & 41.493827 & 35.101790 & 33.805649\end{array}$

$\begin{array}{lrrr}\text { C7-C10-C11-C12 - } 40^{\circ} & & \\ \text { C } & 44.742015 & 39.876286 & 35.778270 \\ \text { C } & 45.056852 & 39.108737 & 34.666168 \\ \text { H } & 45.984738 & 38.569592 & 34.621433 \\ \text { C } & 44.190988 & 39.018202 & 33.594672 \\ \text { H } & 44.438816 & 38.424857 & 32.733251 \\ \text { C } & 42.981611 & 39.702718 & 33.609392 \\ \text { O } & 42.195618 & 39.613455 & 32.535054 \\ \text { H } & 41.284953 & 39.902149 & 32.696188 \\ \text { C } & 42.647077 & 40.456009 & 34.727026 \\ \text { H } & 41.709194 & 40.980294 & 34.742305 \\ \text { C } & 43.526581 & 40.542661 & 35.791938 \\ \text { H } & 43.253888 & 41.155144 & 36.633654 \\ \text { C } & 41.470943 & 35.941991 & 34.503857 \\ \text { H } & 41.149456 & 35.576185 & 35.472885 \\ \text { H } & 42.485375 & 36.314726 & 34.609043 \\ \text { C } & 40.577883 & 37.100856 & 34.067679\end{array}$




\begin{tabular}{|c|c|c|c|}
\hline $\mathrm{H}$ & 40.746678 & 37.944224 & 34.730736 \\
\hline $\mathrm{H}$ & 40.836922 & 37.469975 & 33.082083 \\
\hline C & 39.091499 & 36.835143 & 34.082129 \\
\hline 0 & 38.606367 & 35.784257 & 34.352098 \\
\hline 0 & 38.339403 & 37.868784 & 33.785124 \\
\hline $\mathrm{H}$ & 38.829811 & 38.668014 & 33.544807 \\
\hline $\mathrm{N}$ & 32.753668 & 46.754447 & 29.397500 \\
\hline $\mathrm{H}$ & 33.623602 & 46.286019 & 29.344209 \\
\hline C & 31.586169 & 45.966437 & 29.688056 \\
\hline C & 31.761090 & 44.510807 & 29.195057 \\
\hline$S$ & 32.273385 & 43.233028 & 30.412298 \\
\hline C & 33.907017 & 42.577130 & 29.891736 \\
\hline C & 34.954166 & 43.476486 & 29.508241 \\
\hline C & 36.387655 & 43.092628 & 29.540947 \\
\hline C & 37.026198 & 42.332933 & 30.519379 \\
\hline C & 36.591202 & 41.876028 & 31.802135 \\
\hline C & 37.454983 & 41.159231 & 32.599640 \\
\hline C & 38.795075 & 40.846023 & 32.210029 \\
\hline 0 & 39.569595 & 40.161746 & 32.952014 \\
\hline C & 39.217257 & 41.310222 & 30.913722 \\
\hline C & 38.367541 & 42.017127 & 30.114960 \\
\hline C & 38.614611 & 42.589068 & 28.738513 \\
\hline C & 37.212508 & 43.031428 & 28.267303 \\
\hline $\mathrm{H}$ & 37.237395 & 43.983021 & 27.741914 \\
\hline $\mathrm{H}$ & 36.781071 & 42.299087 & 27.583126 \\
\hline $\mathrm{H}$ & 39.077207 & 41.872349 & 28.067171 \\
\hline $\mathrm{H}$ & 39.280145 & 43.446586 & 28.798316 \\
\hline $\mathrm{H}$ & 40.213833 & 41.061446 & 30.590437 \\
\hline $\mathrm{H}$ & 37.121260 & 40.784209 & 33.551007 \\
\hline $\mathrm{H}$ & 35.594733 & 42.079711 & 32.143692 \\
\hline $\mathrm{H}$ & 34.742040 & 44.500602 & 29.765927 \\
\hline 0 & 34.035393 & 41.379958 & 30.018508 \\
\hline $\mathrm{H}$ & 30.803118 & 44.160525 & 28.838633 \\
\hline $\mathrm{H}$ & 32.442538 & 44.498315 & 28.355315 \\
\hline $\mathrm{H}$ & 30.745806 & 46.367533 & 29.128247 \\
\hline C & 31.125981 & 46.022983 & 31.150372 \\
\hline 0 & 29.979743 & 45.906136 & 31.434752 \\
\hline $\mathrm{H}$ & 32.782602 & 47.719231 & 29.567652 \\
\hline $\mathrm{H}$ & 31.862194 & 46.207968 & 31.873354 \\
\hline $\mathrm{H}$ & 45.471825 & 40.028539 & 36.587247 \\
\hline $\mathrm{H}$ & 41.476146 & 35.090532 & 33.787789 \\
\hline
\end{tabular}

C7-C10-C11-C12 -50

$\begin{array}{llll}C & 44.755868 & 39.897088 & 35.786493\end{array}$ 


\begin{tabular}{|c|c|c|c|}
\hline C & 45.069537 & 39.141813 & 34.666030 \\
\hline $\mathrm{H}$ & 45.998930 & 38.605866 & 34.613310 \\
\hline C & 44.200379 & 39.059701 & 33.596470 \\
\hline $\mathrm{H}$ & 44.446675 & 38.475255 & 32.728590 \\
\hline C & 42.988223 & 39.740093 & 33.620926 \\
\hline 0 & 42.199107 & 39.659806 & 32.550445 \\
\hline $\mathrm{H}$ & 41.280140 & 39.930322 & 32.720150 \\
\hline C & 42.655990 & 40.481915 & 34.747501 \\
\hline $\mathrm{H}$ & 41.715769 & 41.001512 & 34.770679 \\
\hline C & 43.538743 & 40.560565 & 35.809932 \\
\hline $\mathrm{H}$ & 43.267278 & 41.164087 & 36.658581 \\
\hline C & 41.499598 & 35.983270 & 34.513925 \\
\hline $\mathrm{H}$ & 41.191707 & 35.616746 & 35.487269 \\
\hline $\mathrm{H}$ & 42.518850 & 36.347326 & 34.601765 \\
\hline C & 40.607260 & 37.148193 & 34.095803 \\
\hline $\mathrm{H}$ & 40.797583 & 37.992335 & 34.752249 \\
\hline $\mathrm{H}$ & 40.845829 & 37.512888 & 33.103605 \\
\hline C & 39.119820 & 36.893202 & 34.149257 \\
\hline 0 & 38.637741 & 35.853677 & 34.468565 \\
\hline 0 & 38.369342 & 37.918872 & 33.832209 \\
\hline $\mathrm{H}$ & 38.863560 & 38.714877 & 33.567820 \\
\hline $\mathrm{N}$ & 32.775768 & 46.722292 & 29.392161 \\
\hline $\mathrm{H}$ & 33.642660 & 46.248036 & 29.337162 \\
\hline C & 31.599276 & 45.946478 & 29.677594 \\
\hline C & 31.748701 & 44.493441 & 29.172292 \\
\hline S & 32.272826 & 43.198103 & 30.369329 \\
\hline C & 33.892145 & 42.564400 & 29.790236 \\
\hline C & 34.973927 & 43.498091 & 29.578419 \\
\hline C & 36.391880 & 43.095247 & 29.579992 \\
\hline C & 37.037082 & 42.327907 & 30.557913 \\
\hline C & 36.610345 & 41.873928 & 31.838417 \\
\hline C & 37.480045 & 41.158285 & 32.634281 \\
\hline C & 38.816502 & 40.844710 & 32.237766 \\
\hline 0 & 39.596373 & 40.157703 & 32.986236 \\
\hline C & 39.224095 & 41.298917 & 30.940937 \\
\hline C & 38.366445 & 42.003954 & 30.142748 \\
\hline C & 38.602953 & 42.557813 & 28.756202 \\
\hline C & 37.200879 & 43.016265 & 28.297903 \\
\hline $\mathrm{H}$ & 37.229930 & 43.966414 & 27.769271 \\
\hline $\mathrm{H}$ & 36.755076 & 42.287018 & 27.620094 \\
\hline $\mathrm{H}$ & 39.044954 & 41.828751 & 28.080907 \\
\hline $\mathrm{H}$ & 39.285068 & 43.403008 & 28.794325 \\
\hline $\mathrm{H}$ & 40.216182 & 41.046926 & 30.606698 \\
\hline $\mathrm{H}$ & 37.148912 & 40.784363 & 33.587366 \\
\hline
\end{tabular}




$\begin{array}{llll}\mathrm{H} & 35.610824 & 42.067232 & 32.182659 \\ \mathrm{H} & 34.757476 & 44.471378 & 29.985859 \\ \mathrm{O} & 34.002989 & 41.364852 & 29.739461 \\ \mathrm{H} & 30.779683 & 44.154065 & 28.835856 \\ \mathrm{H} & 32.411137 & 44.481354 & 28.317257 \\ \mathrm{H} & 30.764153 & 46.361670 & 29.120077 \\ \mathrm{C} & 31.137079 & 45.995864 & 31.139625 \\ \mathrm{O} & 29.992283 & 45.862211 & 31.421359 \\ \mathrm{H} & 32.809202 & 47.688006 & 29.557426 \\ \mathrm{H} & 31.869487 & 46.190262 & 31.864198 \\ \mathrm{H} & 45.486633 & 40.042748 & 36.595830 \\ \mathrm{H} & 41.484497 & 35.133490 & 33.796088\end{array}$

$\begin{array}{llll}\text { C7-C10-C11-C12 -60 } & & \\ \text { C } & 44.760508 & 39.896848 & 35.785128 \\ \text { C } & 45.078124 & 39.140084 & 34.666319 \\ \text { H } & 46.007451 & 38.603585 & 34.617416 \\ \text { C } & 44.213311 & 39.056789 & 33.593481 \\ \text { H } & 44.463897 & 38.471410 & 32.727234 \\ \text { C } & 43.000147 & 39.736911 & 33.611036 \\ \text { O } & 42.216838 & 39.656326 & 32.537873 \\ \text { H } & 41.291510 & 39.921917 & 32.703161 \\ \text { C } & 42.663080 & 40.478023 & 34.737419 \\ \text { H } & 41.721846 & 40.995728 & 34.756919 \\ \text { C } & 43.542094 & 40.558244 & 35.803071 \\ \text { H } & 43.266496 & 41.161831 & 36.650468 \\ \text { C } & 41.498213 & 35.969798 & 34.510633 \\ \text { H } & 41.181597 & 35.608214 & 35.482982 \\ \text { H } & 42.517829 & 36.331354 & 34.606309 \\ \text { C } & 40.616360 & 37.138949 & 34.080732 \\ \text { H } & 40.799986 & 37.979808 & 34.742626 \\ \text { H } & 40.873930 & 37.507613 & 33.095081 \\ \text { C } & 39.126381 & 36.895429 & 34.107834 \\ \text { O } & 38.631222 & 35.856177 & 34.412022 \\ \text { O } & 38.391465 & 37.928487 & 33.783971 \\ \text { H } & 38.896309 & 38.723880 & 33.522786 \\ \text { N } & 32.770469 & 46.717398 & 29.389889 \\ \text { H } & 33.633805 & 46.236000 & 29.339936 \\ \text { C } & 31.585559 & 45.954613 & 29.674042 \\ \text { C } & 31.707897 & 44.503997 & 29.159381 \\ \text { S } & 32.265932 & 43.201910 & 30.335047 \\ \text { C } & 33.871292 & 42.594751 & 29.691233 \\ \text { C } & 34.985572 & 43.519100 & 29.637746 \\ \text { C } & 36.390113 & 43.091990 & 29.596388\end{array}$




\begin{tabular}{|c|c|c|c|}
\hline C & 37.047286 & 42.324846 & 30.571206 \\
\hline C & 36.637887 & 41.870040 & 31.853426 \\
\hline C & 37.518350 & 41.150988 & 32.636241 \\
\hline C & 38.849165 & 40.838894 & 32.226000 \\
\hline 0 & 39.637191 & 40.140998 & 32.967418 \\
\hline C & 39.240758 & 41.301161 & 30.931230 \\
\hline C & 38.369456 & 42.007359 & 30.142444 \\
\hline C & 38.600520 & 42.578608 & 28.762151 \\
\hline C & 37.197413 & 43.040315 & 28.310881 \\
\hline $\mathrm{H}$ & 37.221843 & 43.996696 & 27.795567 \\
\hline $\mathrm{H}$ & 36.758061 & 42.316421 & 27.624751 \\
\hline $\mathrm{H}$ & 39.037150 & 41.856780 & 28.081079 \\
\hline $\mathrm{H}$ & 39.283314 & 43.422983 & 28.808865 \\
\hline $\mathrm{H}$ & 40.230106 & 41.057004 & 30.582761 \\
\hline $\mathrm{H}$ & 37.197926 & 40.777337 & 33.592654 \\
\hline $\mathrm{H}$ & 35.643822 & 42.066936 & 32.213839 \\
\hline $\mathrm{H}$ & 34.771600 & 44.464803 & 30.103902 \\
\hline 0 & 33.957414 & 41.417547 & 29.451733 \\
\hline $\mathrm{H}$ & 30.725782 & 44.171049 & 28.857494 \\
\hline $\mathrm{H}$ & 32.339436 & 44.489868 & 28.281399 \\
\hline $\mathrm{H}$ & 30.754461 & 46.385183 & 29.121611 \\
\hline C & 31.127859 & 45.998284 & 31.137391 \\
\hline 0 & 29.984571 & 45.860124 & 31.421964 \\
\hline $\mathrm{H}$ & 32.810142 & 47.683235 & 29.553846 \\
\hline $\mathrm{H}$ & 31.862423 & 46.190220 & 31.860459 \\
\hline $\mathrm{H}$ & 45.489168 & 40.044524 & 36.595809 \\
\hline $\mathrm{H}$ & 41.487550 & 35.117797 & 33.795180 \\
\hline \multicolumn{4}{|c|}{$\mathrm{C} 7-\mathrm{C} 10-\mathrm{C} 11-\mathrm{C} 12-70^{\circ}$} \\
\hline C & 44.754284 & 39.880313 & 35.784573 \\
\hline C & 45.069369 & 39.122448 & 34.666127 \\
\hline $\mathrm{H}$ & 45.999582 & 38.587676 & 34.614903 \\
\hline C & 44.200498 & 39.034643 & 33.596790 \\
\hline $\mathrm{H}$ & 44.449212 & 38.447419 & 32.731262 \\
\hline C & 42.985190 & 39.711109 & 33.617345 \\
\hline 0 & 42.198042 & 39.625642 & 32.548641 \\
\hline $\mathrm{H}$ & 41.268267 & 39.884726 & 32.716202 \\
\hline C & 42.651679 & 40.455125 & 34.743584 \\
\hline $\mathrm{H}$ & 41.709114 & 40.970400 & 34.765697 \\
\hline C & 43.534803 & 40.540369 & 35.805097 \\
\hline $\mathrm{H}$ & 43.261421 & 41.145939 & 36.651919 \\
\hline C & 41.480483 & 35.940664 & 34.511714 \\
\hline $\mathrm{H}$ & 41.153459 & 35.572600 & 35.478130 \\
\hline $\mathrm{H}$ & 42.497344 & 36.306291 & 34.621916 \\
\hline
\end{tabular}




\begin{tabular}{|c|c|c|c|}
\hline C & 40.598848 & 37.107171 & 34.075265 \\
\hline $\mathrm{H}$ & 40.760128 & 37.943743 & 34.748603 \\
\hline $\mathrm{H}$ & 40.877368 & 37.487155 & 33.099716 \\
\hline C & 39.109537 & 36.853541 & 34.060485 \\
\hline 0 & 38.612696 & 35.805947 & 34.333969 \\
\hline 0 & 38.378718 & 37.887850 & 33.736238 \\
\hline $\mathrm{H}$ & 38.889326 & 38.689805 & 33.494732 \\
\hline $\mathrm{N}$ & 32.756303 & 46.698195 & 29.366344 \\
\hline $\mathrm{H}$ & 33.611877 & 46.202497 & 29.321366 \\
\hline C & 31.559125 & 45.957352 & 29.656465 \\
\hline C & 31.643736 & 44.508100 & 29.137094 \\
\hline S & 32.267099 & 43.213553 & 30.289122 \\
\hline C & 33.851744 & 42.648717 & 29.557461 \\
\hline C & 35.001678 & 43.519970 & 29.686060 \\
\hline C & 36.388980 & 43.057031 & 29.613463 \\
\hline C & 37.042625 & 42.272733 & 30.578592 \\
\hline C & 36.625540 & 41.799694 & 31.850840 \\
\hline C & 37.507055 & 41.081489 & 32.633631 \\
\hline C & 38.843509 & 40.788760 & 32.230884 \\
\hline 0 & 39.632941 & 40.088963 & 32.976983 \\
\hline C & 39.242970 & 41.268829 & 30.948077 \\
\hline C & 38.369100 & 41.974302 & 30.157089 \\
\hline C & 38.610803 & 42.578610 & 28.791157 \\
\hline C & 37.214074 & 43.065495 & 28.341301 \\
\hline $\mathrm{H}$ & 37.246125 & 44.044106 & 27.868706 \\
\hline $\mathrm{H}$ & 36.782540 & 42.374388 & 27.618178 \\
\hline $\mathrm{H}$ & 39.041314 & 41.868402 & 28.092521 \\
\hline $\mathrm{H}$ & 39.304304 & 43.412706 & 28.860887 \\
\hline $\mathrm{H}$ & 40.241419 & 41.047647 & 30.610873 \\
\hline $\mathrm{H}$ & 37.183791 & 40.695610 & 33.584313 \\
\hline $\mathrm{H}$ & 35.625993 & 41.983207 & 32.204173 \\
\hline $\mathrm{H}$ & 34.794591 & 44.455871 & 30.171495 \\
\hline 0 & 33.896640 & 41.541883 & 29.083321 \\
\hline $\mathrm{H}$ & 30.645420 & 44.177855 & 28.891806 \\
\hline $\mathrm{H}$ & 32.226548 & 44.487717 & 28.226274 \\
\hline $\mathrm{H}$ & 30.731974 & 46.406759 & 29.112781 \\
\hline C & 31.111943 & 46.001302 & 31.124215 \\
\hline 0 & 29.969472 & 45.869916 & 31.415289 \\
\hline $\mathrm{H}$ & 32.810932 & 47.663495 & 29.528300 \\
\hline $\mathrm{H}$ & 31.851899 & 46.187099 & 31.843585 \\
\hline $\mathrm{H}$ & 45.485688 & 40.031133 & 36.592619 \\
\hline $\mathrm{H}$ & 41.483114 & 35.092624 & 33.791694 \\
\hline
\end{tabular}

C7-C10-C11-C $12-80^{\circ}$ 


\begin{tabular}{|c|c|c|c|}
\hline C & 44.727827 & 39.876598 & 35.773816 \\
\hline C & 45.049907 & 39.118425 & 34.657201 \\
\hline $\mathrm{H}$ & 45.985713 & 38.593455 & 34.606599 \\
\hline C & 44.180883 & 39.017306 & 33.589406 \\
\hline $\mathrm{H}$ & 44.434481 & 38.427953 & 32.726716 \\
\hline C & 42.957812 & 39.680657 & 33.608102 \\
\hline 0 & 42.170489 & 39.580291 & 32.540952 \\
\hline $\mathrm{H}$ & 41.237836 & 39.834846 & 32.703566 \\
\hline C & 42.619024 & 40.427012 & 34.731084 \\
\hline $\mathrm{H}$ & 41.670785 & 40.931854 & 34.752850 \\
\hline$C$ & 43.502074 & 40.524708 & 35.792150 \\
\hline $\mathrm{H}$ & 43.222383 & 41.129634 & 36.637430 \\
\hline C & 41.429415 & 35.895318 & 34.512310 \\
\hline $\mathrm{H}$ & 41.093959 & 35.523991 & 35.474252 \\
\hline $\mathrm{H}$ & 42.443289 & 36.265736 & 34.636019 \\
\hline C & 40.550009 & 37.060870 & 34.068023 \\
\hline $\mathrm{H}$ & 40.691644 & 37.889836 & 34.755697 \\
\hline $\mathrm{H}$ & 40.851466 & 37.453716 & 33.104475 \\
\hline C & 39.062330 & 36.802425 & 34.011353 \\
\hline 0 & 38.561851 & 35.750448 & 34.261909 \\
\hline 0 & 38.337403 & 37.834737 & 33.669500 \\
\hline $\mathrm{H}$ & 38.851984 & 38.638934 & 33.438194 \\
\hline $\mathrm{N}$ & 32.696614 & 46.749214 & 29.357052 \\
\hline $\mathrm{H}$ & 33.542544 & 46.236519 & 29.311268 \\
\hline C & 31.485090 & 46.030897 & 29.641969 \\
\hline C & 31.529099 & 44.586781 & 29.116417 \\
\hline$S$ & 32.338255 & 43.350284 & 30.212841 \\
\hline C & 33.844199 & 42.818189 & 29.298508 \\
\hline C & 35.059133 & 43.547172 & 29.602780 \\
\hline C & 36.418067 & 43.014446 & 29.520834 \\
\hline C & 37.045759 & 42.205284 & 30.483144 \\
\hline C & 36.597216 & 41.704958 & 31.734521 \\
\hline C & 37.466149 & 40.990742 & 32.535720 \\
\hline C & 38.820854 & 40.729008 & 32.174333 \\
\hline 0 & 39.599465 & 40.031443 & 32.936977 \\
\hline C & 39.254035 & 41.241875 & 30.916957 \\
\hline C & 38.392412 & 41.942107 & 30.105769 \\
\hline C & 38.682692 & 42.590883 & 28.769695 \\
\hline C & 37.317819 & 43.153916 & 28.308293 \\
\hline $\mathrm{H}$ & 37.386855 & 44.180265 & 27.955415 \\
\hline $\mathrm{H}$ & 36.920290 & 42.563898 & 27.485753 \\
\hline $\mathrm{H}$ & 39.087344 & 41.884589 & 28.051927 \\
\hline $\mathrm{H}$ & 39.416661 & 43.384204 & 28.879935 \\
\hline $\mathrm{H}$ & 40.269304 & 41.052397 & 30.612133 \\
\hline
\end{tabular}




$\begin{array}{llll}\text { H } & 37.120733 & 40.595235 & 33.474694 \\ \text { H } & 35.585887 & 41.872110 & 32.062039 \\ \text { H } & 34.891992 & 44.463191 & 30.137835 \\ \text { O } & 33.783481 & 41.871938 & 28.555162 \\ \text { H } & 30.514131 & 44.235936 & 29.003044 \\ \text { H } & 31.998192 & 44.566480 & 28.142259 \\ \text { H } & 30.664080 & 46.502358 & 29.106392 \\ \text { C } & 31.047244 & 46.061552 & 31.114186 \\ \text { O } & 29.907015 & 45.923271 & 31.411454 \\ \text { H } & 32.769091 & 47.710250 & 29.537224 \\ \text { H } & 31.792323 & 46.234737 & 31.831894 \\ \text { H } & 45.456720 & 40.033896 & 36.582967 \\ \text { H } & 41.446367 & 35.047781 & 33.791165\end{array}$

$\begin{array}{llll}\text { C7-C10-C11-C12 -90 } & & \\ \mathrm{C} & 44.743339 & 39.880177 & 35.776067 \\ \mathrm{C} & 45.053014 & 39.126771 & 34.652867 \\ \mathrm{H} & 45.985725 & 38.597081 & 34.592227 \\ \mathrm{C} & 44.175328 & 39.036651 & 33.591170 \\ \mathrm{H} & 44.419166 & 38.452082 & 32.722486 \\ \mathrm{C} & 42.955727 & 39.705533 & 33.623510 \\ \mathrm{O} & 42.160905 & 39.616196 & 32.561229 \\ \mathrm{H} & 41.229271 & 39.868587 & 32.731544 \\ \mathrm{C} & 42.628602 & 40.445853 & 34.753953 \\ \mathrm{H} & 41.683279 & 40.955577 & 34.785802 \\ \mathrm{C} & 43.520492 & 40.533191 & 35.808446 \\ \mathrm{H} & 43.250611 & 41.133887 & 36.659808 \\ \mathrm{C} & 41.456567 & 35.921658 & 34.523425 \\ \mathrm{H} & 41.131480 & 35.554145 & 35.490582 \\ \mathrm{H} & 42.472018 & 36.291395 & 34.633091 \\ \mathrm{C} & 40.570293 & 37.083978 & 34.085133 \\ \mathrm{H} & 40.721466 & 37.918331 & 34.763754 \\ \mathrm{H} & 40.853350 & 37.469564 & 33.113169 \\ \mathrm{C} & 39.082671 & 36.821053 & 34.057683 \\ \mathrm{O} & 38.590553 & 35.769130 & 34.322825 \\ \mathrm{O} & 38.348293 & 37.851617 & 33.730159 \\ \mathrm{H} & 38.856889 & 38.656204 & 33.490072 \\ \mathrm{~N} & 32.632496 & 46.748596 & 29.365259 \\ \mathrm{H} & 33.470141 & 46.222027 & 29.319808 \\ \mathrm{C} & 31.413794 & 46.048025 & 29.662194 \\ \mathrm{C} & 31.441281 & 44.602838 & 29.139837 \\ \mathrm{~S} & 32.304919 & 43.392453 & 30.220653 \\ \mathrm{C} & 33.769581 & 42.861663 & 29.246485 \\ \mathrm{C} & 35.019420 & 43.484520 & 29.631149\end{array}$




\begin{tabular}{|c|c|c|c|}
\hline C & 36.353519 & 42.908185 & 29.500138 \\
\hline C & 36.999928 & 42.131055 & 30.474703 \\
\hline C & 36.550852 & 41.620546 & 31.721630 \\
\hline C & 37.432640 & 40.941103 & 32.538072 \\
\hline C & 38.800141 & 40.723023 & 32.195449 \\
\hline 0 & 39.589447 & 40.054077 & 32.971403 \\
\hline C & 39.234174 & 41.249956 & 30.943809 \\
\hline C & 38.360663 & 41.917424 & 30.118030 \\
\hline C & 38.649131 & 42.579778 & 28.788298 \\
\hline C & 37.280677 & 43.136680 & 28.323541 \\
\hline $\mathrm{H}$ & 37.333607 & 44.183956 & 28.031496 \\
\hline $\mathrm{H}$ & 36.918463 & 42.590107 & 27.456129 \\
\hline $\mathrm{H}$ & 39.059848 & 41.879970 & 28.065729 \\
\hline $\mathrm{H}$ & 39.378926 & 43.376098 & 28.904056 \\
\hline $\mathrm{H}$ & 40.262199 & 41.103442 & 30.658702 \\
\hline $\mathrm{H}$ & 37.087765 & 40.537846 & 33.473919 \\
\hline $\mathrm{H}$ & 35.529901 & 41.754160 & 32.033881 \\
\hline $\mathrm{H}$ & 34.895160 & 44.415001 & 30.152035 \\
\hline 0 & 33.650822 & 42.019763 & 28.393342 \\
\hline $\mathrm{H}$ & 30.425886 & 44.245476 & 29.062025 \\
\hline $\mathrm{H}$ & 31.879941 & 44.578211 & 28.151882 \\
\hline $\mathrm{H}$ & 30.592976 & 46.527000 & 29.133074 \\
\hline C & 30.989968 & 46.086228 & 31.139673 \\
\hline 0 & 29.849872 & 45.970070 & 31.447234 \\
\hline $\mathrm{H}$ & 32.721151 & 47.708248 & 29.544827 \\
\hline $\mathrm{H}$ & 31.744214 & 46.247298 & 31.851002 \\
\hline $\mathrm{H}$ & 45.479971 & 40.030298 & 36.579621 \\
\hline $\mathrm{H}$ & 41.462041 & 35.071855 & 33.805349 \\
\hline \multicolumn{4}{|c|}{ C7-C10-C11-C12-100 } \\
\hline C & 44.760028 & 39.866793 & 35.790530 \\
\hline C & 45.071123 & 39.112975 & 34.668107 \\
\hline $\mathrm{H}$ & 46.003346 & 38.582901 & 34.609258 \\
\hline C & 44.196059 & 39.023579 & 33.604314 \\
\hline $\mathrm{H}$ & 44.442296 & 38.440189 & 32.735470 \\
\hline C & 42.977473 & 39.694206 & 33.633909 \\
\hline 0 & 42.185719 & 39.607863 & 32.569093 \\
\hline $\mathrm{H}$ & 41.252868 & 39.857951 & 32.739944 \\
\hline C & 42.647582 & 40.433559 & 34.764432 \\
\hline $\mathrm{H}$ & 41.703140 & 40.945247 & 34.793965 \\
\hline C & 43.537427 & 40.520583 & 35.820611 \\
\hline $\mathrm{H}$ & 43.266800 & 41.122871 & 36.670691 \\
\hline C & 41.477329 & 35.919239 & 34.516278 \\
\hline $\mathrm{H}$ & 41.160676 & 35.550109 & 35.485682 \\
\hline
\end{tabular}




\begin{tabular}{|c|c|c|c|}
\hline $\mathrm{H}$ & 42.493948 & 36.288160 & 34.617726 \\
\hline C & 40.588982 & 37.082595 & 34.087427 \\
\hline $\mathrm{H}$ & 40.745918 & 37.916871 & 34.764691 \\
\hline $\mathrm{H}$ & 40.863915 & 37.468882 & 33.113461 \\
\hline C & 39.101540 & 36.820728 & 34.072970 \\
\hline 0 & 38.609973 & 35.770331 & 34.344850 \\
\hline 0 & 38.366052 & 37.852468 & 33.750643 \\
\hline $\mathrm{H}$ & 38.876546 & 38.655251 & 33.509845 \\
\hline $\mathrm{N}$ & 32.650522 & 46.701088 & 29.317324 \\
\hline $\mathrm{H}$ & 33.478401 & 46.157127 & 29.305678 \\
\hline C & 31.421837 & 46.025493 & 29.628124 \\
\hline C & 31.437149 & 44.571169 & 29.133061 \\
\hline S & 32.429664 & 43.433434 & 30.176041 \\
\hline C & 33.796155 & 42.874059 & 29.088633 \\
\hline C & 35.098890 & 43.351045 & 29.510339 \\
\hline C & 36.393786 & 42.699374 & 29.343046 \\
\hline C & 37.044604 & 41.977997 & 30.358432 \\
\hline C & 36.575898 & 41.491845 & 31.607476 \\
\hline C & 37.453827 & 40.866140 & 32.468520 \\
\hline C & 38.836947 & 40.680194 & 32.168805 \\
\hline 0 & 39.620236 & 40.050188 & 32.982308 \\
\hline C & 39.293025 & 41.192916 & 30.920060 \\
\hline C & 38.421332 & 41.806213 & 30.049939 \\
\hline C & 38.723874 & 42.427083 & 28.703144 \\
\hline C & 37.376987 & 43.036520 & 28.238610 \\
\hline $\mathrm{H}$ & 37.446579 & 44.110700 & 28.075388 \\
\hline $\mathrm{H}$ & 37.057983 & 42.600037 & 27.297234 \\
\hline $\mathrm{H}$ & 39.077665 & 41.682647 & 27.996884 \\
\hline $\mathrm{H}$ & 39.500525 & 43.182419 & 28.780888 \\
\hline $\mathrm{H}$ & 40.334559 & 41.079407 & 30.671853 \\
\hline $\mathrm{H}$ & 37.096842 & 40.483234 & 33.407962 \\
\hline $\mathrm{H}$ & 35.544049 & 41.608567 & 31.888378 \\
\hline $\mathrm{H}$ & 35.052738 & 44.289648 & 30.028408 \\
\hline 0 & 33.581079 & 42.152266 & 28.150409 \\
\hline $\mathrm{H}$ & 30.427904 & 44.189123 & 29.138661 \\
\hline $\mathrm{H}$ & 31.806993 & 44.533073 & 28.117810 \\
\hline $\mathrm{H}$ & 30.605935 & 46.502756 & 29.090090 \\
\hline C & 30.998979 & 46.094693 & 31.105105 \\
\hline 0 & 29.856033 & 46.011479 & 31.413568 \\
\hline $\mathrm{H}$ & 32.757200 & 47.660825 & 29.484348 \\
\hline $\mathrm{H}$ & 31.756507 & 46.243640 & 31.815713 \\
\hline $\mathrm{H}$ & 45.495278 & 40.016926 & 36.595077 \\
\hline $\mathrm{H}$ & 41.476481 & 35.071182 & 33.796362 \\
\hline
\end{tabular}




\begin{tabular}{|c|c|c|c|}
\hline \multicolumn{4}{|c|}{ C7-C10-C11-C12 - $110^{\circ}$} \\
\hline C & 44.796962 & 39.863393 & 35.793113 \\
\hline C & 45.115661 & 39.110748 & 34.672233 \\
\hline $\mathrm{H}$ & 46.046715 & 38.578225 & 34.620521 \\
\hline C & 44.249039 & 39.024895 & 33.601365 \\
\hline $\mathrm{H}$ & 44.500502 & 38.442199 & 32.733580 \\
\hline C & 43.031609 & 39.697392 & 33.623047 \\
\hline 0 & 42.247236 & 39.613857 & 32.552908 \\
\hline $\mathrm{H}$ & 41.315050 & 39.866744 & 32.720582 \\
\hline C & 42.693518 & 40.434641 & 34.752466 \\
\hline $\mathrm{H}$ & 41.748890 & 40.946112 & 34.775704 \\
\hline C & 43.574820 & 40.517966 & 35.815685 \\
\hline $\mathrm{H}$ & 43.298128 & 41.117661 & 36.665589 \\
\hline C & 41.533383 & 35.943794 & 34.533337 \\
\hline $\mathrm{H}$ & 41.231990 & 35.577872 & 35.508830 \\
\hline $\mathrm{H}$ & 42.556194 & 36.299999 & 34.612773 \\
\hline C & 40.650128 & 37.116579 & 34.123397 \\
\hline $\mathrm{H}$ & 40.839407 & 37.951847 & 34.791064 \\
\hline $\mathrm{H}$ & 40.899916 & 37.492572 & 33.138991 \\
\hline C & 39.160711 & 36.873097 & 34.161713 \\
\hline 0 & 38.667444 & 35.837198 & 34.481714 \\
\hline 0 & 38.427455 & 37.903249 & 33.830129 \\
\hline $\mathrm{H}$ & 38.941427 & 38.692175 & 33.553861 \\
\hline N & 32.659070 & 46.692988 & 29.271427 \\
\hline $\mathrm{H}$ & 33.483286 & 46.142732 & 29.281476 \\
\hline C & 31.427006 & 46.024976 & 29.584117 \\
\hline C & 31.445681 & 44.566940 & 29.100223 \\
\hline$S$ & 32.528834 & 43.486801 & 30.107448 \\
\hline C & 33.823580 & 42.888504 & 28.959071 \\
\hline C & 35.155780 & 43.221972 & 29.429002 \\
\hline C & 36.415264 & 42.512307 & 29.226897 \\
\hline C & 37.084826 & 41.838739 & 30.267652 \\
\hline C & 36.618403 & 41.378667 & 31.526539 \\
\hline C & 37.504927 & 40.806212 & 32.414295 \\
\hline C & 38.896101 & 40.649030 & 32.130404 \\
\hline 0 & 39.684894 & 40.061913 & 32.968124 \\
\hline C & 39.348824 & 41.140107 & 30.872517 \\
\hline C & 38.467109 & 41.699196 & 29.975891 \\
\hline C & 38.758390 & 42.275486 & 28.609255 \\
\hline C & 37.422572 & 42.921838 & 28.166627 \\
\hline $\mathrm{H}$ & 37.500377 & 44.006582 & 28.096317 \\
\hline $\mathrm{H}$ & 37.120309 & 42.569060 & 27.185926 \\
\hline $\mathrm{H}$ & 39.055036 & 41.495404 & 27.913646 \\
\hline $\mathrm{H}$ & 39.568375 & 42.998014 & 28.637450 \\
\hline
\end{tabular}




$\begin{array}{llll}\mathrm{H} & 40.395284 & 41.050140 & 30.635345 \\ \mathrm{H} & 37.149567 & 40.443622 & 33.362362 \\ \mathrm{H} & 35.580852 & 41.472786 & 31.794040 \\ \mathrm{H} & 35.187505 & 44.184328 & 29.902650 \\ \mathrm{O} & 33.547872 & 42.267945 & 27.968090 \\ \mathrm{H} & 30.447583 & 44.160924 & 29.160947 \\ \mathrm{H} & 31.768285 & 44.525158 & 28.069220 \\ \mathrm{H} & 30.612543 & 46.498989 & 29.041195 \\ \mathrm{C} & 31.001224 & 46.104321 & 31.060152 \\ \mathrm{O} & 29.856438 & 46.037688 & 31.366001 \\ \mathrm{H} & 32.771092 & 47.652254 & 29.436379 \\ \mathrm{H} & 31.758568 & 46.242600 & 31.773264 \\ \mathrm{H} & 45.525828 & 40.011083 & 36.603773 \\ \mathrm{H} & 41.505453 & 35.096129 & 33.813448\end{array}$

$\begin{array}{lrll}\text { C7-C10-C11-C12 -120 } & & \\ \text { C } & 44.831892 & 39.877136 & 35.775350 \\ \text { C } & 45.157508 & 39.122787 & 34.658006 \\ \text { H } & 46.087586 & 38.589029 & 34.613694 \\ \text { C } & 44.297958 & 39.033629 & 33.582042 \\ \text { H } & 44.554396 & 38.447496 & 32.718051 \\ \text { C } & 43.080106 & 39.704402 & 33.596045 \\ \text { O } & 42.297573 & 39.610991 & 32.525192 \\ \text { H } & 41.363191 & 39.850982 & 32.696768 \\ \text { C } & 42.736820 & 40.448371 & 34.719688 \\ \text { H } & 41.791635 & 40.959162 & 34.735949 \\ \mathrm{C} & 43.611125 & 40.534714 & 35.788333 \\ \text { H } & 43.329432 & 41.137175 & 36.634818 \\ \text { C } & 41.639339 & 35.960328 & 34.597997 \\ \text { H } & 41.392472 & 35.608605 & 35.593535 \\ \text { H } & 42.672622 & 36.293117 & 34.607049 \\ \text { C } & 40.757721 & 37.149045 & 34.236816 \\ \text { H } & 41.035847 & 37.996599 & 34.856408 \\ \text { H } & 40.915053 & 37.488338 & 33.220879 \\ \text { C } & 39.277773 & 36.949879 & 34.448294 \\ \text { O } & 38.811034 & 35.969617 & 34.938527 \\ \text { O } & 38.525873 & 37.953084 & 34.077993 \\ \text { H } & 39.021473 & 38.709421 & 33.696414 \\ \text { N } & 32.596482 & 46.749723 & 29.329000 \\ \text { H } & 33.425499 & 46.206041 & 29.337299 \\ \text { C } & 31.374189 & 46.064563 & 29.643144 \\ \text { C } & 31.420040 & 44.605253 & 29.162958 \\ \text { S } & 32.539144 & 43.567055 & 30.168158 \\ \text { C } & 33.779475 & 42.908930 & 29.001145\end{array}$




\begin{tabular}{llll} 
C & 35.124507 & 43.099567 & 29.521364 \\
C & 36.353272 & 42.353167 & 29.257768 \\
C & 37.059415 & 41.700911 & 30.289674 \\
C & 36.622221 & 41.249515 & 31.561748 \\
C & 37.533758 & 40.709699 & 32.443391 \\
C & 38.924366 & 40.578361 & 32.138171 \\
O & 39.737642 & 40.029528 & 32.976139 \\
C & 39.346466 & 41.057925 & 30.865225 \\
C & 38.437757 & 41.583232 & 29.974872 \\
C & 38.684278 & 42.142039 & 28.592418 \\
C & 37.350076 & 42.828627 & 28.212172 \\
H & 37.439515 & 43.915305 & 28.232090 \\
H & 37.032788 & 42.560665 & 27.210358 \\
H & 38.913150 & 41.345212 & 27.889662 \\
H & 39.521277 & 42.833283 & 28.570807 \\
H & 40.390793 & 40.986509 & 30.612586 \\
H & 37.202674 & 40.358334 & 33.404202 \\
H & 35.587909 & 41.330980 & 31.844883 \\
H & 35.236068 & 44.081392 & 29.942482 \\
O & 33.469339 & 42.374311 & 27.972530 \\
H & 30.431148 & 44.177934 & 29.227143 \\
H & 31.742678 & 44.566454 & 28.131903 \\
H & 30.551980 & 46.524660 & 29.100486 \\
C & 30.947342 & 46.140467 & 31.118373 \\
O & 29.802156 & 46.073179 & 31.422966 \\
H & 32.701583 & 47.706532 & 29.511664 \\
H & 31.705078 & 46.276563 & 31.831641 \\
H & 45.554634 & 40.021886 & 36.591873 \\
H & 41.545564 & 35.109396 & 33.887043 \\
& & & \\
\hline
\end{tabular}

\begin{tabular}{|c|c|c|c|}
\hline \multicolumn{4}{|c|}{ C7-C10-C11-C12 - $130^{\circ}$} \\
\hline C & 44.835669 & 39.873813 & 35.790867 \\
\hline C & 45.152957 & 39.116443 & 34.672975 \\
\hline $\mathrm{H}$ & 46.075432 & 38.568899 & 34.630091 \\
\hline C & 44.294282 & 39.042381 & 33.595152 \\
\hline $\mathrm{H}$ & 44.543895 & 38.454864 & 32.730110 \\
\hline C & 43.084629 & 39.728874 & 33.608016 \\
\hline 0 & 42.304858 & 39.650735 & 32.534722 \\
\hline $\mathrm{H}$ & 41.366856 & 39.885573 & 32.703806 \\
\hline C & 42.747507 & 40.470551 & 34.735096 \\
\hline $\mathrm{H}$ & 41.807324 & 40.990361 & 34.753901 \\
\hline C & 43.622112 & 40.544342 & 35.804371 \\
\hline $\mathrm{H}$ & 43.346086 & 41.147456 & 36.652229 \\
\hline C & 41.661001 & 36.004805 & 34.572782 \\
\hline
\end{tabular}




\begin{tabular}{|c|c|c|c|}
\hline $\mathrm{H}$ & 41.432087 & 35.654078 & 35.573111 \\
\hline $\mathrm{H}$ & 42.693637 & 36.338774 & 34.561757 \\
\hline C & 40.768038 & 37.189302 & 34.228430 \\
\hline $\mathrm{H}$ & 41.073210 & 38.045202 & 34.822585 \\
\hline $\mathrm{H}$ & 40.881967 & 37.513304 & 33.201629 \\
\hline C & 39.297593 & 36.999793 & 34.506924 \\
\hline 0 & 38.844089 & 36.031355 & 35.030939 \\
\hline 0 & 38.538242 & 38.004383 & 34.154161 \\
\hline $\mathrm{H}$ & 39.030129 & 38.748087 & 33.746639 \\
\hline $\mathrm{N}$ & 32.507432 & 46.755638 & 29.350979 \\
\hline $\mathrm{H}$ & 33.333879 & 46.208858 & 29.328160 \\
\hline C & 31.289824 & 46.068628 & 29.676943 \\
\hline C & 31.342425 & 44.609309 & 29.196160 \\
\hline$S$ & 32.481468 & 43.583622 & 30.189874 \\
\hline C & 33.684693 & 42.908053 & 28.999602 \\
\hline C & 35.030094 & 42.967218 & 29.565275 \\
\hline C & 36.229116 & 42.178926 & 29.276520 \\
\hline C & 36.976824 & 41.578532 & 30.313448 \\
\hline C & 36.583518 & 41.181051 & 31.618038 \\
\hline C & 37.528229 & 40.689809 & 32.492732 \\
\hline C & 38.910425 & 40.557386 & 32.147952 \\
\hline 0 & 39.751108 & 40.041895 & 32.978632 \\
\hline C & 39.288706 & 40.996238 & 30.848351 \\
\hline C & 38.346578 & 41.469629 & 29.965031 \\
\hline C & 38.543025 & 41.970065 & 28.556355 \\
\hline C & 37.206868 & 42.667800 & 28.216867 \\
\hline $\mathrm{H}$ & 37.301763 & 43.753678 & 28.263869 \\
\hline $\mathrm{H}$ & 36.868116 & 42.427556 & 27.215524 \\
\hline $\mathrm{H}$ & 38.717690 & 41.140669 & 27.877202 \\
\hline $\mathrm{H}$ & 39.395633 & 42.634393 & 28.465429 \\
\hline $\mathrm{H}$ & 40.326148 & 40.929669 & 30.568775 \\
\hline $\mathrm{H}$ & 37.233027 & 40.382389 & 33.479958 \\
\hline $\mathrm{H}$ & 35.558918 & 41.270053 & 31.931930 \\
\hline $\mathrm{H}$ & 35.222949 & 43.979006 & 29.880458 \\
\hline 0 & 33.362354 & 42.478627 & 27.929131 \\
\hline $\mathrm{H}$ & 30.358630 & 44.172261 & 29.265477 \\
\hline $\mathrm{H}$ & 31.660034 & 44.573619 & 28.163468 \\
\hline $\mathrm{H}$ & 30.460140 & 46.523794 & 29.141720 \\
\hline C & 30.875331 & 46.141394 & 31.155953 \\
\hline 0 & 29.734301 & 46.056495 & 31.470949 \\
\hline $\mathrm{H}$ & 32.621657 & 47.709980 & 29.540392 \\
\hline $\mathrm{H}$ & 31.636965 & 46.293920 & 31.861618 \\
\hline $\mathrm{H}$ & 45.560483 & 40.012884 & 36.606454 \\
\hline $\mathrm{H}$ & 41.554290 & 35.153981 & 33.864219 \\
\hline
\end{tabular}




\begin{tabular}{|c|c|c|c|}
\hline \multicolumn{4}{|c|}{ C7-C10-C11-C12-140 } \\
\hline C & 44.841477 & 39.783749 & 35.741791 \\
\hline C & 45.201284 & 38.970351 & 34.677384 \\
\hline $\mathrm{H}$ & 46.121644 & 38.419934 & 34.700911 \\
\hline C & 44.387104 & 38.840288 & 33.571229 \\
\hline $\mathrm{H}$ & 44.668508 & 38.209553 & 32.747843 \\
\hline C & 43.184373 & 39.528876 & 33.507527 \\
\hline 0 & 42.442220 & 39.387756 & 32.402048 \\
\hline $\mathrm{H}$ & 41.535420 & 39.674530 & 32.517763 \\
\hline C & 42.803202 & 40.330508 & 34.573089 \\
\hline $\mathrm{H}$ & 41.869479 & 40.861285 & 34.527461 \\
\hline C & 43.632916 & 40.457828 & 35.674179 \\
\hline $\mathrm{H}$ & 43.324985 & 41.105053 & 36.476796 \\
\hline C & 41.680346 & 35.924387 & 34.584443 \\
\hline $\mathrm{H}$ & 41.476592 & 35.572829 & 35.589045 \\
\hline $\mathrm{H}$ & 42.705392 & 36.277078 & 34.552159 \\
\hline C & 40.755191 & 37.088633 & 34.255229 \\
\hline $\mathrm{H}$ & 41.072691 & 37.962462 & 34.817256 \\
\hline $\mathrm{H}$ & 40.811777 & 37.385129 & 33.215542 \\
\hline C & 39.308396 & 36.879250 & 34.622011 \\
\hline 0 & 38.912283 & 35.973037 & 35.275076 \\
\hline 0 & 38.486229 & 37.817306 & 34.194515 \\
\hline $\mathrm{H}$ & 38.936060 & 38.513727 & 33.711509 \\
\hline $\mathrm{N}$ & 32.532804 & 46.806514 & 29.261553 \\
\hline $\mathrm{H}$ & 33.341778 & 46.242820 & 29.158900 \\
\hline C & 31.282920 & 46.159014 & 29.533068 \\
\hline C & 31.245526 & 44.728947 & 28.981610 \\
\hline$S$ & 32.167731 & 43.478069 & 29.928833 \\
\hline C & 33.776571 & 43.344885 & 29.034941 \\
\hline C & 34.847038 & 43.224944 & 29.942104 \\
\hline C & 36.213690 & 42.856127 & 29.665855 \\
\hline C & 36.954145 & 41.997503 & 30.539270 \\
\hline C & 36.594883 & 41.469662 & 31.812854 \\
\hline C & 37.525297 & 40.750495 & 32.534479 \\
\hline C & 38.858558 & 40.527008 & 32.047948 \\
\hline 0 & 39.702332 & 39.880070 & 32.718931 \\
\hline C & 39.191927 & 41.060820 & 30.741751 \\
\hline C & 38.254140 & 41.749396 & 30.014415 \\
\hline C & 38.392430 & 42.455833 & 28.682431 \\
\hline C & 36.957921 & 42.940240 & 28.354722 \\
\hline $\mathrm{H}$ & 36.939887 & 43.937084 & 27.927873 \\
\hline $\mathrm{H}$ & 36.491137 & 42.280732 & 27.627396 \\
\hline & 38.797579 & 41.811704 & 27.908725 \\
\hline
\end{tabular}




$\begin{array}{llll}\text { H } & 39.074020 & 43.296987 & 28.782342 \\ \mathrm{H} & 40.190158 & 40.893213 & 30.375987 \\ \mathrm{H} & 37.273018 & 40.338276 & 33.494545 \\ \mathrm{H} & 35.609768 & 41.625303 & 32.207508 \\ \mathrm{H} & 34.566416 & 43.224282 & 30.974608 \\ \mathrm{O} & 33.781894 & 43.368347 & 27.812625 \\ \mathrm{H} & 30.217009 & 44.401652 & 28.991241 \\ \mathrm{H} & 31.602224 & 44.710755 & 27.960764 \\ \mathrm{H} & 30.485111 & 46.690132 & 29.016625 \\ \mathrm{C} & 30.853819 & 46.167441 & 31.007086 \\ \mathrm{O} & 29.707377 & 46.076896 & 31.309948 \\ \mathrm{H} & 32.663003 & 47.758695 & 29.449189 \\ \mathrm{H} & 31.609273 & 46.287925 & 31.725324 \\ \mathrm{H} & 45.534140 & 39.959681 & 36.577881 \\ \mathrm{H} & 41.574622 & 35.071494 & 33.878273\end{array}$

$\begin{array}{lrrr}\text { C7-C10-C11-C12 -150 } & & \\ \mathrm{C} & 44.838165 & 39.781984 & 35.747368 \\ \mathrm{C} & 45.195510 & 38.966789 & 34.683551 \\ \mathrm{H} & 46.115100 & 38.414823 & 34.706645 \\ \mathrm{C} & 44.379580 & 38.836582 & 33.578531 \\ \mathrm{H} & 44.658358 & 38.203678 & 32.755941 \\ \mathrm{C} & 43.178643 & 39.527574 & 33.516494 \\ \mathrm{O} & 42.431741 & 39.386388 & 32.413070 \\ \mathrm{H} & 41.528980 & 39.678348 & 32.537740 \\ \mathrm{C} & 42.800584 & 40.332217 & 34.580616 \\ \mathrm{H} & 41.867830 & 40.864607 & 34.535463 \\ \mathrm{C} & 43.631262 & 40.458735 & 35.680762 \\ \mathrm{H} & 43.325525 & 41.107021 & 36.483276 \\ \mathrm{C} & 41.687761 & 35.924119 & 34.599330 \\ \mathrm{H} & 41.488265 & 35.555496 & 35.598688 \\ \mathrm{H} & 42.711358 & 36.280624 & 34.569767 \\ \mathrm{C} & 40.757693 & 37.089640 & 34.292309 \\ \mathrm{H} & 41.077622 & 37.957278 & 34.862473 \\ \mathrm{H} & 40.805408 & 37.397587 & 33.255606 \\ \mathrm{C} & 39.314399 & 36.871505 & 34.666721 \\ \mathrm{O} & 38.924847 & 35.956556 & 35.310701 \\ \mathrm{O} & 38.487014 & 37.812472 & 34.254124 \\ \mathrm{H} & 38.934841 & 38.516923 & 33.782699 \\ \mathrm{~N} & 32.496119 & 46.820521 & 29.270093 \\ \mathrm{H} & 33.292558 & 46.254120 & 29.107639 \\ \mathrm{C} & 31.242349 & 46.183478 & 29.544423 \\ \mathrm{C} & 31.192326 & 44.756733 & 28.988063 \\ \mathrm{~S} & 32.077308 & 43.487345 & 29.942349\end{array}$




\begin{tabular}{|c|c|c|c|}
\hline C & 33.720509 & 43.401773 & 29.107094 \\
\hline C & 34.745211 & 43.121147 & 30.030989 \\
\hline C & 36.106740 & 42.793138 & 29.752691 \\
\hline C & 36.880740 & 41.946496 & 30.623718 \\
\hline C & 36.541925 & 41.414366 & 31.898462 \\
\hline C & 37.492442 & 40.7213 & 32.619503 \\
\hline C & 38.827474 & 40.530669 & 32.125457 \\
\hline 0 & 39.688398 & 39.901689 & 32.791261 \\
\hline C & 39.140612 & 41.069076 & 30.815879 \\
\hline C & 38.179751 & 41.729979 & 30.089314 \\
\hline C & 38.288187 & 42.421285 & 28.744906 \\
\hline C & 36.84525 & 42.901916 & 28.43928 \\
\hline $\mathrm{H}$ & 36.820513 & 43.906463 & 28.031942 \\
\hline $\mathrm{H}$ & 36.376377 & 42.257181 & 27.699417 \\
\hline $\mathrm{H}$ & 38.673496 & 41.762466 & 27.970395 \\
\hline $\mathrm{H}$ & 38.974288 & 43.262372 & 28.810089 \\
\hline $\mathrm{H}$ & 40.138149 & 40.918261 & 30.441801 \\
\hline $\mathrm{H}$ & 37.254303 & 40.304315 & 33.580826 \\
\hline $\mathrm{H}$ & 35.55722 & 41.550425 & 32.300362 \\
\hline $\mathrm{H}$ & 34.428003 & 43.002466 & 31.046331 \\
\hline 0 & 33.78188 & 43.592503 & 27.901053 \\
\hline $\mathrm{H}$ & 30.159552 & 44.444534 & 28.979218 \\
\hline $\mathrm{H}$ & 31.568696 & 44.735983 & 27.974525 \\
\hline $\mathrm{H}$ & 30.443858 & 46.724057 & 29.038752 \\
\hline C & 30.828296 & 46.184575 & 31.02253 \\
\hline 0 & 29.68479 & 46.096541 & 31.337946 \\
\hline $\mathrm{H}$ & 32.637998 & 47.769652 & 29.462779 \\
\hline $\mathrm{H}$ & 31.592489 & 46.297573 & 31.732633 \\
\hline $\mathrm{H}$ & 45.531878 & 39.957142 & 36.582442 \\
\hline $\mathrm{H}$ & 41.582476 & 35.082007 & 33.880339 \\
\hline \multicolumn{4}{|c|}{ C7-C10-C11-C12 - $150^{\circ}$} \\
\hline C & 44.842622 & 39.786958 & 35.756592 \\
\hline C & 45.197725 & 38.970857 & 34.692809 \\
\hline $\mathrm{H}$ & 46.114611 & 38.414656 & 34.717047 \\
\hline C & 44.382971 & 38.845167 & 33.586407 \\
\hline $\mathrm{H}$ & 44.660559 & 38.212328 & 32.763384 \\
\hline C & 43.185154 & 39.541195 & 33.523543 \\
\hline 0 & 42.439554 & 39.405011 & 32.418121 \\
\hline $\mathrm{H}$ & 41.53896 & 39.701913 & 32.543014 \\
\hline C & 42.808196 & 40.345297 & 34.588441 \\
\hline $\mathrm{H}$ & 41.877492 & 40.881189 & 34.542813 \\
\hline C & 43.638084 & 40.467892 & 35.689626 \\
\hline $\mathrm{H}$ & 43.333735 & 41.116381 & 36.492492 \\
\hline
\end{tabular}




\begin{tabular}{|c|c|c|c|}
\hline C & 41.694944 & 35.934356 & 34.602338 \\
\hline $\mathrm{H}$ & 41.495765 & 35.564748 & 35.601495 \\
\hline $\mathrm{H}$ & 42.718278 & 36.291577 & 34.572942 \\
\hline C & 40.763726 & 37.098866 & 34.296294 \\
\hline $\mathrm{H}$ & 41.078673 & 37.964703 & 34.871942 \\
\hline $\mathrm{H}$ & 40.816401 & 37.411703 & 33.261062 \\
\hline C & 39.318834 & 36.873844 & 34.660795 \\
\hline 0 & 38.925544 & 35.948586 & 35.287191 \\
\hline 0 & 38.492769 & 37.821152 & 34.259249 \\
\hline $\mathrm{H}$ & 38.942497 & 38.529319 & 33.796423 \\
\hline $\mathrm{N}$ & 32.46351 & 46.832715 & 29.26306 \\
\hline $\mathrm{H}$ & 33.243574 & 46.264899 & 29.035975 \\
\hline C & 31.209685 & 46.203812 & 29.550605 \\
\hline$C$ & 31.146451 & 44.781109 & 28.986279 \\
\hline$S$ & 32.028863 & 43.492461 & 29.913356 \\
\hline C & 33.708871 & 43.515927 & 29.161467 \\
\hline C & 34.684872 & 43.067723 & 30.073231 \\
\hline C & 36.044935 & 42.771014 & 29.797864 \\
\hline C & 36.842293 & 41.936743 & 30.66826 \\
\hline C & 36.517391 & 41.388165 & 31.937939 \\
\hline C & 37.483998 & 40.708635 & 32.653345 \\
\hline C & 38.820617 & 40.547285 & 32.153593 \\
\hline 0 & 39.693795 & 39.924982 & 32.809597 \\
\hline C & 39.120199 & 41.105128 & 30.849881 \\
\hline C & 38.145267 & 41.756454 & 30.132423 \\
\hline$C$ & 38.24094 & 42.464805 & 28.796475 \\
\hline C & 36.786741 & 42.905993 & 28.487475 \\
\hline $\mathrm{H}$ & 36.734262 & 43.907545 & 28.079078 \\
\hline $\mathrm{H}$ & 36.338618 & 42.247672 & 27.745484 \\
\hline $\mathrm{H}$ & 38.657251 & 41.831289 & 28.016268 \\
\hline $\mathrm{H}$ & 38.898329 & 43.326701 & 28.881863 \\
\hline $\mathrm{H}$ & 40.120109 & 40.978551 & 30.472754 \\
\hline $\mathrm{H}$ & 37.25633 & 40.28112 & 33.612551 \\
\hline $\mathrm{H}$ & 35.532374 & 41.502746 & 32.345662 \\
\hline $\mathrm{H}$ & 34.3263 & 42.823221 & 31.053288 \\
\hline 0 & 33.838132 & 43.904551 & 28.005993 \\
\hline $\mathrm{H}$ & 30.112743 & 44.47179 & 28.9908 \\
\hline $\mathrm{H}$ & 31.508275 & 44.77034 & 27.96768 \\
\hline $\mathrm{H}$ & 30.405787 & 46.749675 & 29.058677 \\
\hline C & 30.810634 & 46.196152 & 31.032866 \\
\hline 0 & 29.66974 & 46.106054 & 31.357653 \\
\hline $\mathrm{H}$ & 32.619935 & 47.776742 & 29.467737 \\
\hline $\mathrm{H}$ & 31.580714 & 46.305496 & 31.737119 \\
\hline $\mathrm{H}$ & 45.536416 & 39.959363 & 36.592168 \\
\hline
\end{tabular}




\begin{tabular}{|c|c|c|c|}
\hline $\mathrm{H}$ & 41.589714 & 35.09293 & 33.882692 \\
\hline \multicolumn{4}{|c|}{ C7-C10-C11-C12 - $170^{\circ}$} \\
\hline C & 44.860021 & 39.79203 & 35.76155 \\
\hline C & 45.216997 & 38.972382 & 34.701172 \\
\hline $\mathrm{H}$ & 46.132176 & 38.414176 & 34.730539 \\
\hline C & 44.406043 & 38.845111 & 33.592396 \\
\hline $\mathrm{H}$ & 44.685622 & 38.210324 & 32.771521 \\
\hline C & 43.209082 & 39.542249 & 33.524389 \\
\hline 0 & 42.467597 & 39.405022 & 32.4169 \\
\hline $\mathrm{H}$ & 41.568689 & 39.710253 & 32.53579 \\
\hline C & 42.828894 & 40.347959 & 34.586997 \\
\hline $\mathrm{H}$ & 41.898519 & 40.884071 & 34.537755 \\
\hline C & 43.656009 & 40.473135 & 35.689967 \\
\hline $\mathrm{H}$ & 43.349575 & 41.123301 & 36.490705 \\
\hline C & 41.703363 & 35.925709 & 34.592985 \\
\hline $\mathrm{H}$ & 41.504737 & 35.569244 & 35.59693 \\
\hline $\mathrm{H}$ & 42.727514 & 36.279997 & 34.557443 \\
\hline C & 40.776376 & 37.090386 & 34.273543 \\
\hline $\mathrm{H}$ & 41.090632 & 37.959956 & 34.843961 \\
\hline $\mathrm{H}$ & 40.835498 & 37.395988 & 33.236204 \\
\hline C & 39.32885 & 36.872316 & 34.631348 \\
\hline 0 & 38.925861 & 35.947287 & 35.251291 \\
\hline 0 & 38.510304 & 37.827617 & 34.231221 \\
\hline $\mathrm{H}$ & 38.967759 & 38.534134 & 33.775306 \\
\hline$N$ & 32.467306 & 46.857425 & 29.256645 \\
\hline $\mathrm{H}$ & 33.24264 & 46.288806 & 29.01359 \\
\hline C & 31.215148 & 46.226341 & 29.541473 \\
\hline C & 31.164914 & 44.803911 & 28.975488 \\
\hline$S$ & 32.076575 & 43.526407 & 29.887458 \\
\hline C & 33.766133 & 43.607303 & 29.166392 \\
\hline C & 34.706643 & 43.016407 & 30.032451 \\
\hline C & 36.066671 & 42.7403 & 29.762599 \\
\hline C & 36.879649 & 41.939337 & 30.654609 \\
\hline C & 36.554539 & 41.383894 & 31.919519 \\
\hline C & 37.527036 & 40.717377 & 32.640024 \\
\hline C & 38.867701 & 40.572712 & 32.146153 \\
\hline 0 & 39.742388 & 39.950483 & 32.800172 \\
\hline C & 39.170773 & 41.146622 & 30.850962 \\
\hline C & 38.191692 & 41.790578 & 30.132999 \\
\hline C & 38.286869 & 42.510889 & 28.804269 \\
\hline C & 36.822824 & 42.885478 & 28.462885 \\
\hline $\mathrm{H}$ & 36.734268 & 43.872789 & 28.029101 \\
\hline $\mathrm{H}$ & 36.41897 & 42.185878 & 27.731122 \\
\hline
\end{tabular}




$\begin{array}{rrrr}\text { H } & 38.749308 & 41.901148 & 28.035364 \\ \mathrm{H} & 38.899481 & 43.402151 & 28.913963 \\ \mathrm{H} & 40.176166 & 41.040104 & 30.482079 \\ \mathrm{H} & 37.299016 & 40.282279 & 33.595579 \\ \mathrm{H} & 35.566 & 41.483214 & 32.322702 \\ \mathrm{H} & 34.324952 & 42.701783 & 30.983711 \\ \mathrm{O} & 33.927241 & 44.133524 & 28.070268 \\ \mathrm{H} & 30.13608 & 44.476559 & 28.991925 \\ \mathrm{H} & 31.514789 & 44.804111 & 27.953042 \\ \mathrm{H} & 30.407459 & 46.767352 & 29.049633 \\ \mathrm{C} & 30.813182 & 46.207266 & 31.022347 \\ \mathrm{O} & 29.671244 & 46.113575 & 31.343515 \\ \mathrm{H} & 32.62904 & 47.799418 & 29.466005 \\ \mathrm{H} & 31.581703 & 46.308661 & 31.72948 \\ \mathrm{H} & 45.550614 & 39.964339 & 36.599688 \\ \mathrm{H} & 41.594871 & 35.075863 & 33.883759\end{array}$

The QM(CASSCF)/MM calculated minimum-energy isomerization path connecting both S0-cisP-MIN and S0-cisT-MIN of the single-bond-rotation locked PYP chromophore in the R52A mutated protein.

$\begin{array}{llll}\text { Point 1 } & & & \\ \text { C } & 44.729395 & 39.903951 & 35.757692 \\ \text { C } & 45.012988 & 39.197336 & 34.595171 \\ \text { H } & 45.946224 & 38.676580 & 34.479123 \\ \text { C } & 44.106667 & 39.161626 & 33.556503 \\ \text { H } & 44.337670 & 38.634170 & 32.649947 \\ \text { C } & 42.897252 & 39.845040 & 33.646836 \\ \text { O } & 42.086523 & 39.812140 & 32.594117 \\ \text { H } & 41.167719 & 40.084126 & 32.763914 \\ \text { C } & 42.604346 & 40.544533 & 34.812711 \\ \text { H } & 41.671349 & 41.072369 & 34.885819 \\ \text { C } & 43.518421 & 40.576551 & 35.849738 \\ \text { H } & 43.277370 & 41.150247 & 36.728617 \\ \text { C } & 41.436905 & 35.974530 & 34.469859 \\ \text { H } & 41.099377 & 35.653122 & 35.450056 \\ \text { H } & 42.460976 & 36.319438 & 34.575991 \\ \text { C } & 40.574389 & 37.144541 & 33.980748 \\ \text { H } & 40.727515 & 37.994274 & 34.638810 \\ \text { H } & 40.885451 & 37.501387 & 33.003462 \\ \text { C } & 39.079320 & 36.906825 & 33.924857 \\ \text { O } & 38.568415 & 35.845492 & 34.108934 \\ \text { O } & 38.346031 & 37.961709 & 33.670983 \\ \text { H } & 38.825661 & 38.784270 & 33.454145\end{array}$




$\begin{array}{llll}\text { N } & 32.629921 & 46.790775 & 29.404567 \\ \text { H } & 33.483120 & 46.290789 & 29.345688 \\ \text { C } & 31.429116 & 46.043036 & 29.597085 \\ \text { C } & 31.412830 & 44.740048 & 28.773981 \\ \text { S } & 32.092763 & 43.135341 & 29.376261 \\ \text { C } & 33.948418 & 43.284149 & 29.499992 \\ \text { C } & 34.664011 & 42.304842 & 28.727964 \\ \text { C } & 35.969797 & 41.871775 & 28.930487 \\ \text { C } & 36.791804 & 41.767553 & 30.076970 \\ \text { C } & 36.472713 & 41.805855 & 31.462603 \\ \text { C } & 37.366524 & 41.327022 & 32.389430 \\ \text { C } & 38.674055 & 40.832101 & 32.035980 \\ \text { O } & 39.441297 & 40.306503 & 32.911836 \\ \text { C } & 39.006509 & 40.869032 & 30.642781 \\ \text { C } & 38.084267 & 41.292937 & 29.717488 \\ \text { C } & 38.196712 & 41.266040 & 28.214317 \\ \text { C } & 36.723693 & 41.213688 & 27.782407 \\ \text { H } & 36.545332 & 41.678019 & 26.822845 \\ \text { H } & 36.405197 & 40.178667 & 27.706888 \\ \text { H } & 38.774602 & 40.435659 & 27.829595 \\ \text { H } & 38.657402 & 42.178973 & 27.843584 \\ \text { H } & 39.968234 & 40.493824 & 30.336547 \\ \text { H } & 37.095823 & 41.295036 & 33.428062 \\ \text { H } & 35.497213 & 42.115602 & 31.789407 \\ \text { H } & 34.146995 & 41.970689 & 27.849056 \\ \text { O } & 34.395088 & 44.135236 & 30.199228 \\ \text { H } & 30.381213 & 44.468668 & 28.600815 \\ \text { H } & 31.862275 & 44.954221 & 27.816835 \\ \text { H } & 30.598916 & 46.626094 & 29.204569 \\ \text { C } & 31.031689 & 45.854182 & 31.059440 \\ \text { O } & 29.925882 & 45.547434 & 31.373434 \\ \text { H } & 32.695353 & 47.746753 & 29.596495 \\ \text { H } & 31.766383 & 46.084169 & 31.772241 \\ \text { H } & 45.496897 & 40.030327 & 36.537232 \\ \text { H } & 41.425163 & 35.093730 & 33.789555\end{array}$

Point 2

$\begin{array}{llll}\text { C } & 44.775214 & 39.899147 & 35.753469 \\ \text { C } & 45.040457 & 39.180077 & 34.594549 \\ \text { H } & 45.962631 & 38.640448 & 34.478212 \\ \text { C } & 44.129865 & 39.154463 & 33.560581 \\ \text { H } & 44.345078 & 38.613365 & 32.658473 \\ \text { C } & 42.929501 & 39.853082 & 33.654247 \\ \text { O } & 42.116979 & 39.825451 & 32.604493\end{array}$




\begin{tabular}{|c|c|c|c|}
\hline $\mathrm{H}$ & 41.195400 & 40.095689 & 32.768145 \\
\hline C & 42.651948 & 40.559442 & 34.820617 \\
\hline $\mathrm{H}$ & 41.725124 & 41.096899 & 34.899542 \\
\hline C & 43.573054 & 40.586422 & 35.852129 \\
\hline $\mathrm{H}$ & 43.342405 & 41.163431 & 36.731577 \\
\hline C & 41.464489 & 35.983640 & 34.483364 \\
\hline $\mathrm{H}$ & 41.132281 & 35.661386 & 35.464748 \\
\hline $\mathrm{H}$ & 42.491411 & 36.320977 & 34.581211 \\
\hline C & 40.605022 & 37.157698 & 33.999377 \\
\hline $\mathrm{H}$ & 40.772913 & 38.009203 & 34.651559 \\
\hline $\mathrm{H}$ & 40.904737 & 37.508064 & 33.016343 \\
\hline C & 39.108248 & 36.928735 & 33.962415 \\
\hline 0 & 38.594384 & 35.875056 & 34.178669 \\
\hline 0 & 38.378650 & 37.981326 & 33.690288 \\
\hline $\mathrm{H}$ & 38.863081 & 38.796723 & 33.459888 \\
\hline $\mathrm{N}$ & 32.655113 & 46.789088 & 29.350261 \\
\hline $\mathrm{H}$ & 33.519634 & 46.310719 & 29.295670 \\
\hline C & 31.472605 & 46.010169 & 29.531402 \\
\hline C & 31.512225 & 44.697005 & 28.717277 \\
\hline$S$ & 32.139247 & 43.091637 & 29.372954 \\
\hline C & 34.006576 & 43.177067 & 29.539094 \\
\hline C & 34.723654 & 42.264121 & 28.697641 \\
\hline C & 36.029028 & 41.829922 & 28.900789 \\
\hline C & 36.843472 & 41.724693 & 30.052577 \\
\hline C & 36.512428 & 41.749340 & 31.434198 \\
\hline C & 37.411187 & 41.289965 & 32.364707 \\
\hline C & 38.725646 & 40.816223 & 32.015559 \\
\hline 0 & 39.491076 & 40.295369 & 32.894922 \\
\hline C & 39.072084 & 40.873403 & 30.627401 \\
\hline C & 38.147026 & 41.281548 & 29.696918 \\
\hline C & 38.272016 & 41.275159 & 28.193623 \\
\hline C & 36.804199 & 41.210687 & 27.745523 \\
\hline $\mathrm{H}$ & 36.627029 & 41.698699 & 26.798234 \\
\hline $\mathrm{H}$ & 36.498421 & 40.174634 & 27.638510 \\
\hline $\mathrm{H}$ & 38.866053 & 40.459784 & 27.801480 \\
\hline $\mathrm{H}$ & 38.724672 & 42.199640 & 27.842237 \\
\hline $\mathrm{H}$ & 40.042078 & 40.515322 & 30.328526 \\
\hline $\mathrm{H}$ & 37.133118 & 41.245977 & 33.400956 \\
\hline $\mathrm{H}$ & 35.531676 & 42.048244 & 31.752113 \\
\hline $\mathrm{H}$ & 34.216338 & 41.973559 & 27.799166 \\
\hline 0 & 34.453110 & 43.925963 & 30.346259 \\
\hline $\mathrm{H}$ & 30.494448 & 44.427101 & 28.474498 \\
\hline $\mathrm{H}$ & 32.030209 & 44.898506 & 27.792458 \\
\hline $\mathrm{H}$ & 30.638388 & 46.561367 & 29.105285 \\
\hline
\end{tabular}




$\begin{array}{llll}\mathrm{C} & 31.042343 & 45.815054 & 30.981480 \\ \mathrm{O} & 29.937553 & 45.472483 & 31.254562 \\ \mathrm{H} & 32.705181 & 47.744406 & 29.560170 \\ \mathrm{H} & 31.741894 & 46.071832 & 31.719853 \\ \mathrm{H} & 45.545463 & 40.006154 & 36.532140 \\ \mathrm{H} & 41.441025 & 35.103831 & 33.801529\end{array}$

Point 3

\begin{tabular}{llll}
$\mathrm{C}$ & 44.813601 & 39.877892 & 35.744277 \\
$\mathrm{C}$ & 45.082409 & 39.155815 & 34.588957 \\
$\mathrm{H}$ & 46.001709 & 38.611701 & 34.482564 \\
$\mathrm{C}$ & 44.179493 & 39.132074 & 33.549394 \\
$\mathrm{H}$ & 44.399720 & 38.590209 & 32.649563 \\
$\mathrm{C}$ & 42.978485 & 39.829973 & 33.637264 \\
$\mathrm{O}$ & 42.169840 & 39.800651 & 32.585539 \\
$\mathrm{H}$ & 41.249059 & 40.082110 & 32.748788 \\
$\mathrm{C}$ & 42.694450 & 40.536289 & 34.802784 \\
$\mathrm{H}$ & 41.766409 & 41.071771 & 34.878115 \\
$\mathrm{C}$ & 43.611393 & 40.566029 & 35.838071 \\
$\mathrm{H}$ & 43.375414 & 41.142255 & 36.716545 \\
$\mathrm{C}$ & 41.501281 & 35.990044 & 34.491470 \\
$\mathrm{H}$ & 41.179880 & 35.667600 & 35.476196 \\
$\mathrm{H}$ & 42.534203 & 36.311244 & 34.574804 \\
$\mathrm{C}$ & 40.648025 & 37.174938 & 34.025423 \\
$\mathrm{H}$ & 40.838820 & 38.025659 & 34.671908 \\
$\mathrm{H}$ & 40.932548 & 37.522092 & 33.036879 \\
$\mathrm{C}$ & 39.148484 & 36.962439 & 34.021400 \\
$\mathrm{O}$ & 38.627557 & 35.917507 & 34.262818 \\
$\mathrm{O}$ & 38.424854 & 38.019348 & 33.748730 \\
$\mathrm{H}$ & 38.920906 & 38.824443 & 33.511560 \\
$\mathrm{~N}$ & 32.652497 & 46.808092 & 29.319386 \\
$\mathrm{H}$ & 33.522011 & 46.341558 & 29.246367 \\
$\mathrm{C}$ & 31.487958 & 46.007282 & 29.492257 \\
$\mathrm{C}$ & 31.594163 & 44.681291 & 28.698199 \\
$\mathrm{~S}$ & 32.170658 & 43.093017 & 29.434036 \\
$\mathrm{C}$ & 34.041695 & 43.120797 & 29.655520 \\
$\mathrm{C}$ & 34.780565 & 42.292995 & 28.749707 \\
$\mathrm{C}$ & 36.081714 & 41.847941 & 28.953110 \\
$\mathrm{C}$ & 36.885905 & 41.695052 & 30.110372 \\
$\mathrm{C}$ & 36.546846 & 41.652413 & 31.488431 \\
$\mathrm{C}$ & 37.465124 & 41.217416 & 32.411492 \\
$\mathrm{C}$ & 38.797249 & 40.807038 & 32.054175 \\
$\mathrm{C}$ & 39.581205 & 40.302052 & 32.927330 \\
\hline & 39.144008 & 40.916717 & 30.669548
\end{tabular}




$\begin{array}{llll}\text { C } & 38.205986 & 41.312537 & 29.747009 \\ \text { C } & 38.342469 & 41.358630 & 28.246485 \\ \text { C } & 36.880950 & 41.301922 & 27.778046 \\ \text { H } & 36.713916 & 41.845653 & 26.860672 \\ \text { H } & 36.585240 & 40.272360 & 27.600624 \\ \text { H } & 38.948449 & 40.560441 & 27.835618 \\ \text { H } & 38.792055 & 42.298517 & 27.934008 \\ \text { H } & 40.126076 & 40.600684 & 30.363398 \\ \text { H } & 37.178635 & 41.116289 & 33.441696 \\ \text { H } & 35.556940 & 41.902798 & 31.813826 \\ \text { H } & 34.292877 & 42.068170 & 27.822610 \\ \text { O } & 34.457639 & 43.759235 & 30.564153 \\ \text { H } & 30.599058 & 44.402457 & 28.383080 \\ \text { H } & 32.175772 & 44.881012 & 27.809302 \\ \text { H } & 30.644759 & 46.530384 & 29.048799 \\ \text { C } & 31.042670 & 45.809220 & 30.936248 \\ \text { O } & 29.938235 & 45.453540 & 31.181941 \\ \text { H } & 32.696462 & 47.761060 & 29.541080 \\ \text { H } & 31.723741 & 46.075059 & 31.692282 \\ \text { H } & 45.579603 & 39.981232 & 36.526669 \\ \text { H } & 41.453697 & 35.113624 & 33.805847\end{array}$

Point 4

$\begin{array}{llll}\text { C } & 44.840839 & 39.858825 & 35.735264 \\ \text { C } & 45.115279 & 39.119509 & 34.592944 \\ \text { H } & 46.019493 & 38.546160 & 34.510443 \\ \text { C } & 44.219240 & 39.084988 & 33.545960 \\ \text { H } & 44.437004 & 38.519219 & 32.659617 \\ \text { C } & 43.024188 & 39.794085 & 33.613042 \\ \text { O } & 42.214084 & 39.748553 & 32.561337 \\ \text { H } & 41.288622 & 40.011127 & 32.745495 \\ \text { C } & 42.738279 & 40.526498 & 34.761706 \\ \text { H } & 41.811519 & 41.066734 & 34.818522 \\ \text { C } & 43.646086 & 40.562664 & 35.804246 \\ \text { H } & 43.406296 & 41.150691 & 36.674040 \\ \text { C } & 41.541420 & 35.975294 & 34.485997 \\ \text { H } & 41.238311 & 35.642788 & 35.473555 \\ \text { H } & 42.576988 & 36.293254 & 34.553881 \\ \text { C } & 40.679093 & 37.160684 & 34.050685 \\ \text { H } & 40.881763 & 38.007562 & 34.697953 \\ \text { H } & 40.935147 & 37.515320 & 33.057535 \\ \text { C } & 39.181627 & 36.938689 & 34.089002 \\ \text { O } & 38.675533 & 35.893362 & 34.360299 \\ \text { O } & 38.448386 & 37.987169 & 33.814655\end{array}$




\begin{tabular}{llll} 
H & 38.950122 & 38.791413 & 33.574472 \\
N & 32.695099 & 46.799360 & 29.346629 \\
H & 33.567648 & 46.333402 & 29.306857 \\
C & 31.518130 & 46.001476 & 29.488337 \\
C & 31.658039 & 44.643664 & 28.753874 \\
S & 32.164010 & 43.109723 & 29.637353 \\
C & 34.031354 & 43.092202 & 29.910889 \\
C & 34.831121 & 42.426246 & 28.919659 \\
C & 36.134903 & 41.984991 & 29.112366 \\
C & 36.927890 & 41.738197 & 30.264720 \\
C & 36.574176 & 41.562893 & 31.630174 \\
C & 37.495700 & 41.082677 & 32.528183 \\
C & 38.847648 & 40.755331 & 32.159466 \\
O & 39.651416 & 40.228797 & 33.006476 \\
C & 39.200291 & 40.975691 & 30.790145 \\
C & 38.257710 & 41.410515 & 29.889233 \\
C & 38.415331 & 41.566105 & 28.397636 \\
C & 36.959864 & 41.555541 & 27.907386 \\
H & 36.810394 & 42.187557 & 27.045807 \\
H & 36.666393 & 40.548734 & 27.623396 \\
H & 39.014232 & 40.784708 & 27.944127 \\
H & 38.888254 & 42.515763 & 28.157627 \\
H & 40.191322 & 40.706761 & 30.468613 \\
H & 37.202024 & 40.878324 & 33.541567 \\
H & 35.575660 & 41.758100 & 31.963880 \\
H & 34.366012 & 42.296799 & 27.962217 \\
O & 34.398026 & 43.561234 & 30.927151 \\
H & 30.679768 & 44.360829 & 28.393563 \\
H & 32.299913 & 44.778872 & 27.895579 \\
H & 30.694449 & 46.517039 & 29.002321 \\
C & 31.016965 & 45.835195 & 30.924545 \\
O & 29.896797 & 45.513652 & 31.149560 \\
H & 32.725951 & 47.754590 & 29.559248 \\
& 31.691325 & 46.082948 & 31.693926 \\
\hline & 45.596315 & 39.966790 & 36.526647 \\
H & 41.479189 & 35.106715 & 33.793453
\end{tabular}

\section{Point 5}

$\begin{array}{llll}\text { C } & 44.833353 & 39.829294 & 35.749338 \\ \text { C } & 45.129259 & 39.078748 & 34.620633 \\ \text { H } & 46.038734 & 38.513386 & 34.557730 \\ \text { C } & 44.251539 & 39.029926 & 33.558767 \\ \text { H } & 44.485474 & 38.453914 & 32.683294 \\ \text { C } & 43.053038 & 39.733382 & 33.601235\end{array}$




\begin{tabular}{|c|c|c|c|}
\hline 0 & 42.258620 & 39.680321 & 32.538406 \\
\hline$H$ & 41.334908 & 39.950240 & 32.721915 \\
\hline C & 42.741671 & 40.472090 & 34.739550 \\
\hline H & 41.809023 & 41.003377 & 34.777853 \\
\hline C & 43.631562 & 40.523739 & 35.795079 \\
\hline $\mathrm{H}$ & 43.373771 & 41.118380 & 36.654955 \\
\hline C & 41.557543 & 35.957909 & 34.480607 \\
\hline $\mathrm{H}$ & 41.261323 & 35.619289 & 35.468156 \\
\hline $\mathrm{H}$ & 42.593423 & 36.275274 & 34.544666 \\
\hline C & 40.694508 & 37.143441 & 34.055716 \\
\hline $\mathrm{H}$ & 40.906214 & 37.990124 & 34.700913 \\
\hline H & 40.948133 & 37.499075 & 33.062555 \\
\hline C & 39.196980 & 36.929093 & 34.106648 \\
\hline 0 & 38.686035 & 35.888443 & 34.385896 \\
\hline 0 & 38.470354 & 37.981522 & 33.831505 \\
\hline $\mathrm{H}$ & 38.985117 & 38.779508 & 33.601614 \\
\hline $\mathrm{N}$ & 32.742242 & 46.755450 & 29.365215 \\
\hline $\mathrm{H}$ & 33.612968 & 46.280274 & 29.381054 \\
\hline C & 31.560572 & 45.963800 & 29.480140 \\
\hline C & 31.743099 & 44.594851 & 28.781166 \\
\hline S & 32.197192 & 43.122796 & 29.771196 \\
\hline C & 34.050912 & 43.047599 & 30.085598 \\
\hline C & 34.923519 & 42.606554 & 29.030715 \\
\hline C & 36.235696 & 42.188951 & 29.220379 \\
\hline C & 36.995799 & 41.819201 & 30.365306 \\
\hline C & 36.614917 & 41.476824 & 31.692040 \\
\hline C & 37.534815 & 40.960294 & 32.569866 \\
\hline C & 38.912298 & 40.746069 & 32.218738 \\
\hline 0 & 39.725045 & 40.204337 & 33.045427 \\
\hline C & 39.286658 & 41.104104 & 30.886668 \\
\hline C & 38.351074 & 41.590407 & 30.005993 \\
\hline C & 38.562422 & 41.930356 & 28.554039 \\
\hline C & 37.130331 & 41.988419 & 28.004315 \\
\hline $\mathrm{H}$ & 37.015150 & 42.778028 & 27.278435 \\
\hline $\mathrm{H}$ & 36.865262 & 41.052806 & 27.518614 \\
\hline $\mathrm{H}$ & 39.181516 & 41.206091 & 28.036321 \\
\hline $\mathrm{H}$ & 39.043870 & 42.900743 & 28.457050 \\
\hline $\mathrm{H}$ & 40.298610 & 40.918279 & 30.571710 \\
\hline $\mathrm{H}$ & 37.222107 & 40.652244 & 33.551185 \\
\hline $\mathrm{H}$ & 35.603120 & 41.587010 & 32.017801 \\
\hline $\mathrm{H}$ & 34.517812 & 42.641278 & 28.038038 \\
\hline 0 & 34.354390 & 43.286563 & 31.199721 \\
\hline $\mathrm{H}$ & 30.790264 & 44.296178 & 28.369622 \\
\hline $\mathrm{H}$ & 32.435162 & 44.719755 & 27.960615 \\
\hline
\end{tabular}




$\begin{array}{llll}\text { H } & 30.752280 & 46.464356 & 28.955087 \\ \mathrm{C} & 31.022274 & 45.837360 & 30.907433 \\ \mathrm{O} & 29.888164 & 45.558164 & 31.117470 \\ \mathrm{H} & 32.761018 & 47.712892 & 29.568993 \\ \mathrm{H} & 31.691882 & 46.073240 & 31.686784 \\ \mathrm{H} & 45.575490 & 39.954905 & 36.550381 \\ \mathrm{H} & 41.491797 & 35.093193 & 33.785284\end{array}$

Point 6

$\begin{array}{llll}\mathrm{C} & 44.808102 & 39.855712 & 35.755998 \\ \mathrm{C} & 45.123350 & 39.117642 & 34.624093 \\ \mathrm{H} & 46.046380 & 38.573964 & 34.558475 \\ \mathrm{C} & 44.250271 & 39.059989 & 33.557460 \\ \mathrm{H} & 44.504027 & 38.503584 & 32.674855 \\ \mathrm{C} & 43.037929 & 39.740555 & 33.599883 \\ \mathrm{O} & 42.249867 & 39.686017 & 32.528916 \\ \mathrm{H} & 41.326741 & 39.957257 & 32.702930 \\ \mathrm{C} & 42.703454 & 40.455937 & 34.745544 \\ \mathrm{H} & 41.759524 & 40.967015 & 34.785712 \\ \mathrm{C} & 43.590007 & 40.520270 & 35.803900 \\ \mathrm{H} & 43.315907 & 41.104747 & 36.666031 \\ \mathrm{C} & 41.557389 & 35.957692 & 34.466754 \\ \mathrm{H} & 41.265585 & 35.617141 & 35.454929 \\ \mathrm{H} & 42.592783 & 36.277086 & 34.528244 \\ \mathrm{C} & 40.685077 & 37.136636 & 34.043019 \\ \mathrm{H} & 40.895058 & 37.991189 & 34.678401 \\ \mathrm{H} & 40.925281 & 37.486576 & 33.044859 \\ \mathrm{C} & 39.191535 & 36.901837 & 34.101420 \\ \mathrm{O} & 38.697958 & 35.858908 & 34.401251 \\ \mathrm{O} & 38.446157 & 37.936937 & 33.807769 \\ \mathrm{H} & 38.948021 & 38.733533 & 33.555509 \\ \mathrm{~N} & 32.744487 & 46.706317 & 29.395319 \\ \mathrm{H} & 33.596010 & 46.189256 & 29.399103 \\ \mathrm{C} & 31.539305 & 45.955930 & 29.543311 \\ \mathrm{C} & 31.669336 & 44.589222 & 28.836657 \\ \mathrm{~S} & 32.029720 & 43.140279 & 29.874912 \\ \mathrm{C} & 33.830898 & 43.023694 & 30.293076 \\ \mathrm{C} & 34.817082 & 42.880499 & 29.251786 \\ \mathrm{C} & 36.143319 & 42.507662 & 29.426811 \\ \mathrm{C} & 36.902579 & 41.980205 & 30.510928 \\ \mathrm{C} & 36.528382 & 41.451456 & 31.776635 \\ \mathrm{C} & 37.467274 & 40.883912 & 32.600644 \\ \mathrm{O} & 38.856593 & 40.772566 & 32.242955 \\ & 39.684812 & 40.184358 & 33.015888\end{array}$




$\begin{array}{llll}\text { C } & 39.223223 & 41.313085 & 30.971800 \\ \text { C } & 38.273396 & 41.874941 & 30.151865 \\ \text { C } & 38.496864 & 42.469649 & 28.785289 \\ \text { C } & 37.074658 & 42.598005 & 28.223060 \\ \text { H } & 36.939000 & 43.529912 & 27.687451 \\ \text { H } & 36.852375 & 41.783478 & 27.538806 \\ \text { H } & 39.145232 & 41.867660 & 28.157364 \\ \text { H } & 38.952002 & 43.450467 & 28.880512 \\ \text { H } & 40.249836 & 41.238205 & 30.657507 \\ \text { H } & 37.165003 & 40.459831 & 33.541400 \\ \text { H } & 35.509286 & 41.471147 & 32.095675 \\ \text { H } & 34.477857 & 43.104620 & 28.257570 \\ \text { O } & 33.991242 & 42.981825 & 31.462555 \\ \text { H } & 30.711690 & 44.330624 & 28.408142 \\ \text { H } & 32.378127 & 44.683624 & 28.026603 \\ \text { H } & 30.730939 & 46.485972 & 29.048334 \\ \text { C } & 31.065213 & 45.837745 & 30.993148 \\ \text { O } & 29.930923 & 45.612365 & 31.260265 \\ \text { H } & 32.789775 & 47.665254 & 29.596924 \\ \text { H } & 31.790084 & 46.028744 & 31.735503 \\ \text { H } & 45.543502 & 39.990577 & 36.561322 \\ \text { H } & 41.490188 & 35.093937 & 33.769467\end{array}$

Point 7

$\begin{array}{llll}\text { C } & 44.825842 & 39.829682 & 35.744850 \\ \text { C } & 45.135283 & 39.091270 & 34.611292 \\ \text { H } & 46.055594 & 38.544562 & 34.542260 \\ \text { C } & 44.261990 & 39.036600 & 33.546344 \\ \text { H } & 44.512686 & 38.479494 & 32.663382 \\ \text { C } & 43.052260 & 39.720864 & 33.590016 \\ \text { O } & 42.265846 & 39.664481 & 32.519253 \\ \text { H } & 41.342610 & 39.935623 & 32.681872 \\ \text { C } & 42.722409 & 40.437391 & 34.736280 \\ \text { H } & 41.780792 & 40.952954 & 34.778426 \\ \text { C } & 43.610632 & 40.499339 & 35.794139 \\ \text { H } & 43.340861 & 41.086628 & 36.655423 \\ \text { C } & 41.552117 & 35.968686 & 34.496701 \\ \text { H } & 41.267917 & 35.632271 & 35.488402 \\ \text { H } & 42.584520 & 36.298185 & 34.550601 \\ \text { C } & 40.668368 & 37.136644 & 34.065908 \\ \text { H } & 40.874180 & 38.000835 & 34.689187 \\ \text { H } & 40.898175 & 37.476121 & 33.061353 \\ \text { C } & 39.177904 & 36.890438 & 34.132429 \\ \text { O } & 38.692679 & 35.850377 & 34.453610\end{array}$




\begin{tabular}{llll} 
O & 38.427025 & 37.915404 & 33.817059 \\
H & 38.931279 & 38.705158 & 33.549530 \\
N & 32.721359 & 46.715274 & 29.398720 \\
H & 33.568155 & 46.191583 & 29.422533 \\
C & 31.509042 & 45.973564 & 29.542764 \\
C & 31.663936 & 44.582566 & 28.890143 \\
S & 31.976654 & 43.178234 & 30.002141 \\
C & 33.764863 & 42.967844 & 30.418628 \\
C & 34.756759 & 42.938723 & 29.373305 \\
C & 36.082632 & 42.551779 & 29.505522 \\
C & 36.854453 & 41.961496 & 30.545759 \\
C & 36.498284 & 41.379086 & 31.793219 \\
C & 37.452333 & 40.799474 & 32.589396 \\
C & 38.840395 & 40.725594 & 32.218222 \\
O & 39.683558 & 40.134015 & 32.968481 \\
C & 39.186492 & 41.310369 & 30.961224 \\
C & 38.221883 & 41.887802 & 30.170623 \\
C & 38.431714 & 42.539338 & 28.828264 \\
C & 37.001872 & 42.724423 & 28.298239 \\
H & 36.862598 & 43.692158 & 27.827591 \\
H & 36.762917 & 41.960294 & 27.563180 \\
H & 39.055353 & 41.951574 & 28.163773 \\
H & 38.919039 & 43.499029 & 28.958312 \\
H & 40.212278 & 41.264628 & 30.638732 \\
H & 37.163163 & 40.346083 & 33.520239 \\
H & 35.482737 & 41.370014 & 32.119537 \\
H & 34.422246 & 43.268002 & 28.407542 \\
O & 33.902656 & 42.747649 & 31.572196 \\
H & 30.727659 & 44.310897 & 28.423721 \\
H & 32.412474 & 44.647461 & 28.114063 \\
H & 30.709616 & 46.486829 & 29.016634 \\
H & 30.999854 & 45.886860 & 30.985704 \\
\hline & 29.858177 & 45.680131 & 31.235468 \\
H & 32.778012 & 47.675344 & 29.588151 \\
\hline & 45.567012 & 39.963526 & 36.546644 \\
H & & 35.100605 & 33.804186
\end{tabular}

\section{Point 8}

$\begin{array}{llll}\text { C } & 44.810992 & 39.813151 & 35.731140 \\ \text { C } & 45.121603 & 39.075829 & 34.596651 \\ \text { H } & 46.040465 & 38.527324 & 34.524566 \\ \text { C } & 44.251327 & 39.026767 & 33.530661 \\ \text { H } & 44.501712 & 38.472032 & 32.646584\end{array}$




\begin{tabular}{|c|c|c|c|}
\hline C & 43.045601 & 39.716908 & 33.571573 \\
\hline 0 & 42.266125 & 39.665077 & 32.497992 \\
\hline $\mathrm{H}$ & 41.347186 & 39.943191 & 32.649411 \\
\hline$C$ & 42.713913 & 40.432668 & 34.718307 \\
\hline $\mathrm{H}$ & 41.775555 & 40.954224 & 34.758995 \\
\hline$C$ & 43.598797 & 40.488473 & 35.778784 \\
\hline $\mathrm{H}$ & 43.330720 & 41.076744 & 36.639961 \\
\hline C & 41.545388 & 35.969289 & 34.506540 \\
\hline $\mathrm{H}$ & 41.258133 & 35.637525 & 35.498388 \\
\hline $\mathrm{H}$ & 42.577842 & 36.298310 & 34.559238 \\
\hline C & 40.664822 & 37.138455 & 34.070722 \\
\hline $\mathrm{H}$ & 40.873056 & 38.003250 & 34.693506 \\
\hline $\mathrm{H}$ & 40.894429 & 37.476366 & 33.065474 \\
\hline C & 39.175061 & 36.897083 & 34.139531 \\
\hline 0 & 38.686918 & 35.865531 & 34.480739 \\
\hline 0 & 38.426703 & 37.918244 & 33.805314 \\
\hline $\mathrm{H}$ & 38.930770 & 38.702769 & 33.528607 \\
\hline $\mathrm{N}$ & 32.693550 & 46.717027 & 29.397793 \\
\hline $\mathrm{H}$ & 33.529881 & 46.179644 & 29.482865 \\
\hline C & 31.477685 & 45.986639 & 29.529516 \\
\hline C & 31.642083 & 44.595647 & 28.875310 \\
\hline$S$ & 31.921037 & 43.188481 & 29.994316 \\
\hline C & 33.695035 & 42.812292 & 30.335012 \\
\hline C & 34.713366 & 42.941619 & 29.332713 \\
\hline C & 36.043255 & 42.566132 & 29.474703 \\
\hline C & 36.810527 & 41.922766 & 30.486662 \\
\hline C & 36.463325 & 41.296465 & 31.717194 \\
\hline C & 37.426091 & 40.728076 & 32.506345 \\
\hline C & 38.817619 & 40.709738 & 32.152539 \\
\hline 0 & 39.669479 & 40.136152 & 32.900150 \\
\hline C & 39.158028 & 41.329814 & 30.911845 \\
\hline C & 38.185914 & 41.900025 & 30.126808 \\
\hline C & 38.401300 & 42.602055 & 28.812741 \\
\hline C & 36.975963 & 42.831856 & 28.296143 \\
\hline $\mathrm{H}$ & 36.838072 & 43.829231 & 27.894759 \\
\hline $\mathrm{H}$ & 36.741425 & 42.119351 & 27.509883 \\
\hline $\mathrm{H}$ & 39.005639 & 42.024868 & 28.121189 \\
\hline $\mathrm{H}$ & 38.918604 & 43.540360 & 28.973297 \\
\hline $\mathrm{H}$ & 40.188437 & 41.323211 & 30.600626 \\
\hline $\mathrm{H}$ & 37.139733 & 40.243367 & 33.421623 \\
\hline $\mathrm{H}$ & 35.448734 & 41.240476 & 32.032506 \\
\hline $\mathrm{H}$ & 34.415166 & 43.381437 & 28.399704 \\
\hline 0 & 33.793691 & 42.358742 & 31.428556 \\
\hline $\mathrm{H}$ & 30.726095 & 44.332858 & 28.365189 \\
\hline
\end{tabular}




$\begin{array}{llll}\mathrm{H} & 32.425243 & 44.661744 & 28.133434 \\ \mathrm{H} & 30.688142 & 46.504160 & 28.993650 \\ \mathrm{C} & 30.966595 & 45.920739 & 30.973474 \\ \mathrm{O} & 29.826357 & 45.718784 & 31.231668 \\ \mathrm{H} & 32.755613 & 47.682228 & 29.563532 \\ \mathrm{H} & 31.677214 & 46.128840 & 31.722193 \\ \mathrm{H} & 45.556688 & 39.952567 & 36.528684 \\ \mathrm{H} & 41.481408 & 35.099134 & 33.816850\end{array}$

Point 9

$\begin{array}{llll}\mathrm{C} & 44.777152 & 39.824364 & 35.741233 \\ \mathrm{C} & 45.090548 & 39.094916 & 34.601577 \\ \mathrm{H} & 46.013950 & 38.553529 & 34.522964 \\ \mathrm{C} & 44.217976 & 39.047152 & 33.536638 \\ \mathrm{H} & 44.470128 & 38.499777 & 32.648362 \\ \mathrm{C} & 43.009746 & 39.732425 & 33.583292 \\ \mathrm{O} & 42.226948 & 39.686209 & 32.510789 \\ \mathrm{H} & 41.312366 & 39.967691 & 32.670616 \\ \mathrm{C} & 42.676946 & 40.440316 & 34.733988 \\ \mathrm{H} & 41.736769 & 40.957870 & 34.777812 \\ \mathrm{C} & 43.562446 & 40.493581 & 35.793688 \\ \mathrm{H} & 43.293769 & 41.076560 & 36.658100 \\ \mathrm{C} & 41.541912 & 35.965368 & 34.508750 \\ \mathrm{H} & 41.258545 & 35.630689 & 35.500716 \\ \mathrm{H} & 42.574246 & 36.295819 & 34.559457 \\ \mathrm{C} & 40.659625 & 37.136862 & 34.082210 \\ \mathrm{H} & 40.873883 & 37.997077 & 34.709561 \\ \mathrm{H} & 40.882355 & 37.478192 & 33.076493 \\ \mathrm{C} & 39.170905 & 36.895592 & 34.160472 \\ \mathrm{O} & 38.685699 & 35.863622 & 34.502546 \\ \mathrm{O} & 38.418039 & 37.915512 & 33.831568 \\ \mathrm{H} & 38.910893 & 38.705968 & 33.552495 \\ \mathrm{~N} & 32.636489 & 46.723717 & 29.419267 \\ \mathrm{H} & 33.470750 & 46.187554 & 29.481061 \\ \mathrm{C} & 31.419175 & 46.001527 & 29.579369 \\ \mathrm{C} & 31.574316 & 44.596093 & 28.949927 \\ \mathrm{~S} & 31.826010 & 43.207763 & 30.100655 \\ \mathrm{C} & 33.578692 & 42.711271 & 30.354158 \\ \mathrm{C} & 34.620401 & 42.950111 & 29.395770 \\ \mathrm{C} & 35.952527 & 42.585319 & 29.540651 \\ \mathrm{C} & 36.727618 & 41.938942 & 30.545273 \\ \mathrm{C} & 36.393963 & 41.301542 & 31.774204 \\ \mathrm{C} & 37.366634 & 40.743234 & 32.558082 \\ \mathrm{C} & 38.757754 & 40.746011 & 32.202806\end{array}$




$\begin{array}{llll}\text { O } & 39.619067 & 40.169701 & 32.934957 \\ \text { C } & 39.086236 & 41.387743 & 30.969915 \\ \text { C } & 38.104817 & 41.948775 & 30.190306 \\ \text { C } & 38.312446 & 42.672430 & 28.887378 \\ \text { C } & 36.886237 & 42.900757 & 28.374479 \\ \text { H } & 36.732076 & 43.907402 & 28.000577 \\ \text { H } & 36.664745 & 42.209126 & 27.566770 \\ \text { H } & 38.918921 & 42.108818 & 28.186956 \\ \text { H } & 38.824709 & 43.610155 & 29.059083 \\ \text { H } & 40.116944 & 41.407747 & 30.659570 \\ \text { H } & 37.088010 & 40.251390 & 33.472053 \\ \text { H } & 35.381594 & 41.226938 & 32.091597 \\ \text { H } & 34.336811 & 43.450110 & 28.489291 \\ \text { O } & 33.644931 & 42.112178 & 31.378844 \\ \text { H } & 30.663801 & 44.340862 & 28.426854 \\ \text { H } & 32.367938 & 44.640026 & 28.218085 \\ \text { H } & 30.620330 & 46.500596 & 29.041217 \\ \text { C } & 30.943304 & 45.960481 & 31.034719 \\ \text { O } & 29.806544 & 45.787191 & 31.331375 \\ \text { H } & 32.708075 & 47.690080 & 29.572399 \\ \text { H } & 31.685037 & 46.145822 & 31.759849 \\ \text { H } & 45.523900 & 39.966569 & 36.537154 \\ \text { H } & 41.476596 & 35.096713 & 33.816535\end{array}$

Point 10

$\begin{array}{llll}\text { C } & 44.727897 & 39.809392 & 35.749458 \\ \text { C } & 45.059746 & 39.064201 & 34.624959 \\ \text { H } & 45.986517 & 38.523531 & 34.573686 \\ \text { C } & 44.205316 & 39.005277 & 33.544430 \\ \text { H } & 44.472103 & 38.444280 & 32.668782 \\ \text { C } & 42.999742 & 39.697767 & 33.562193 \\ \text { O } & 42.228133 & 39.644209 & 32.478687 \\ \text { H } & 41.315851 & 39.934265 & 32.633876 \\ \text { C } & 42.650829 & 40.422412 & 34.696258 \\ \text { H } & 41.712560 & 40.944937 & 34.716685 \\ \text { C } & 43.515697 & 40.484456 & 35.771613 \\ \text { H } & 43.232298 & 41.080326 & 36.622385 \\ \text { C } & 41.553419 & 35.959105 & 34.490046 \\ \text { H } & 41.283995 & 35.629351 & 35.487748 \\ \text { H } & 42.586238 & 36.289736 & 34.523363 \\ \text { C } & 40.666959 & 37.130468 & 34.069474 \\ \text { H } & 40.893550 & 37.993625 & 34.687377 \\ \text { H } & 40.877424 & 37.461992 & 33.057918 \\ \text { C } & 39.176234 & 36.897245 & 34.176894\end{array}$




\begin{tabular}{llll} 
O & 38.690999 & 35.870636 & 34.533076 \\
O & 38.421837 & 37.919652 & 33.855398 \\
H & 38.912352 & 38.707227 & 33.568605 \\
N & 32.634183 & 46.809794 & 29.413302 \\
H & 33.483423 & 46.294163 & 29.436151 \\
C & 31.424214 & 46.071253 & 29.608192 \\
C & 31.574942 & 44.655395 & 28.997173 \\
S & 31.825116 & 43.292418 & 30.181282 \\
C & 33.554278 & 42.703018 & 30.374192 \\
C & 34.604887 & 42.992683 & 29.440654 \\
C & 35.931940 & 42.606581 & 29.566035 \\
C & 36.708814 & 41.937930 & 30.554075 \\
C & 36.380492 & 41.309396 & 31.790180 \\
C & 37.357004 & 40.744904 & 32.567680 \\
C & 38.747815 & 40.747807 & 32.202796 \\
O & 39.615827 & 40.173513 & 32.927194 \\
C & 39.067275 & 41.388150 & 30.966359 \\
C & 38.083096 & 41.947881 & 30.191583 \\
C & 38.286624 & 42.668792 & 28.886131 \\
C & 36.857857 & 42.924164 & 28.394035 \\
H & 36.715432 & 43.934866 & 28.025896 \\
H & 36.613822 & 42.242528 & 27.584857 \\
H & 38.864378 & 42.086252 & 28.176587 \\
H & 38.827155 & 43.593339 & 29.043476 \\
H & 40.097104 & 41.411069 & 30.653459 \\
H & 37.082741 & 40.255799 & 33.485002 \\
H & 35.367860 & 41.240711 & 32.112264 \\
H & 34.329329 & 43.539483 & 28.559310 \\
O & 33.595337 & 42.024682 & 31.350513 \\
H & 30.663247 & 44.393000 & 28.479294 \\
H & 32.365769 & 44.673562 & 28.261618 \\
H & 30.609263 & 46.562985 & 29.088430 \\
C & 30.984755 & 46.030101 & 31.076299 \\
H & 29.851866 & 45.887381 & 31.402858 \\
\hline & 32.691731 & 47.775130 & 29.564201 \\
\hline & 31.754127 & 46.174460 & 31.780811 \\
H & 41.478201 & 35.087371 & 33.802078
\end{tabular}

